\title{
THE PHYSICOCHEMICAL PROPERTIES AND CELLULAR TOXICITY OF VARIABLY SYNTHESIZED ZINC OXIDE NANOPARTICLES
}

\author{
By \\ Catherine Binns Anders
}

\author{
A dissertation \\ submitted in partial fulfillment \\ of the requirements for the degree of \\ Doctor of Philosophy in Biomolecular Sciences \\ Boise State University
}

December 2017 
(C)2017

Catherine Binns Anders

ALL RIGHTS RESERVED 


\title{
BOISE STATE UNIVERSITY GRADUATE COLLEGE \\ DEFENSE COMMITTEE AND FINAL READING APPROVALS
}

\author{
of the dissertation submitted by \\ Catherine Binns Anders
}

Dissertation Title: The Physicochemical Properties and Cellular Toxicity of Variably Synthesized Zinc Oxide Nanoparticles

Date of Final Oral Examination: 08 December 2017

The following individuals read and discussed the dissertation submitted by student Catherine Binns Anders, and they evaluated her presentation and response to questions during the oral examination. They found the student passed the oral examination.

Denise G. Wingett, Ph.D. Chair, Supervisory Committee

Daniel Fologea, Ph.D. Member, Supervisory Committee

Matthew L. Ferguson, Ph.D. $\quad$ Member, Supervisory Committee

Dmitri Tenne, Ph.D. Member, Supervisory Committee

Sam Lohse, Ph.D. $\quad$ External Examiner

The final reading approval of the dissertation was granted by Denise G. Wingett, Ph.D., Chair, Supervisory Committee. The dissertation was approved by the Graduate College. 


\section{DEDICATION}

I dedicate this in memory of Dr. Alex Punnoose. You were an incredible mentor, a gifted scientist, and an uncommonly kind and genuine person. The world is a better place because of you. 


\section{ACKNOWLEGEMENTS}

This endeavor would never have been possible without the support of a multitude of people. First, I would like to thank Dr. Denise Wingett for her unending support and for being the best mentor a student could ever ask for. Many thanks to my committee members, Dr. Daniel Fologea, Dr. Matt Ferguson and Dr. Dmitri Tenne, for their support and assistance. A special thanks to Josh Eixenberger and Jordan Chess for brainstorming and troubleshooting sessions, help with countless experiments, and hours of laughter. To John Rasmussen, Mahdu Kongara and Rebecca Hermann for all your help and answers to my never-ending stream of questions.

Finally, I am forever grateful for the unconditional love and support of my family and friends. I wish I could thank you all personally but there will never be enough words. Know that I love you all deeply. Duane, you are my husband, father of my children, best friend, and my soul mate. You make my life work. Helen, your faith in me has often been my strength; thank you for reminding me daily of God's presence in my life. Susannah, you truly are the sunshine and music in my life. Thank you for your infectious smile and joyful spirit. Clayton, you remind me that perseverance is worth it. Thank you for your goofiness, utter nonsense, and for believing in me. Brent, you will always be my doodle and quiet science guy. Thank you for loving science as much as I do, our long walks and thoughtful conversations. Beth, you are my rock - I love you sis! Finally, to Beth Gee and Cheri Lamb, I really don't know what I would have done without either of you. Thanks for the late-night conversations, endless laughs and unwavering friendship. 


\begin{abstract}
Nanotechnology has grown exponentially since its inception in the early 1970's. Since then, bionanotechnological devices and treatment options have significantly improved disease treatments and patient outcomes; however, this rapid growth in consumer related products has also prompted concern. Zinc oxide nanoparticles (nZnO), known for their inherent toxicity and prevalent global use in consumer products and medical applications, have received much of this attention. Significant research efforts have focused on both toxicity remediation through material property modification and the exploitation of these same factors to create potential cancer therapeutics. There is general agreement that the physicochemical properties of $\mathrm{nZnO}$ strongly contribute to NP-induced toxicity; however, inconsistencies in the material property characterization methods employed, and an understanding of how those properties influence cytotoxicity in mammalian cells has led to discrepancies in the literature. Additionally, more research is needed to connect the material properties of $\mathrm{nZnO}$ to downstream cellular responses. Here, a panel of variably synthesized $\mathrm{nZnO}$ was utilized to thoroughly investigate the material properties of the particles as they relate to cytotoxicity, oxidative stress, and transcriptome changes in different mammalian cell types. The goals of this study are three-fold: $i$ ) reduce NP agglomeration and sedimentation tendencies within complex media and achieve dispersion stability, $i i$ ) define which material property interactions have the greatest potential to affect cellular toxicity, and to iii) examine the preferential toxicity of nZnO towards Jurkat leukemic cells through genetic expression studies.
\end{abstract}


Chapter 2 highlights the importance of dispersion stability and the effect of fetal bovine serum (FBS) proteins on the dispersion stability, dosimetry and NP-induced cytotoxicity of $\mathrm{nZnO}$ in suspension and adherent in vitro cell culture models. The presence of surface adsorbed proteins from the FBS on the $\mathrm{nZnO}$ decreased agglomeration and sedimentation potential. Furthermore, FBS-stabilized $\mathrm{nZnO}$ dispersions resulted in toxicity increases in suspension cells when compared to unstable dispersions; however, toxicity was decreased in adherent cell models with stable dispersions. These observations indicate that improved dispersion stability leads to increased NP bioavailability for suspension cells and reduced NP sedimentation onto adherent cell layers resulting in more accurate in vitro toxicity assessments.

In Chapter 3, we utilized an expanded panel of $\mathrm{nZnO}$ synthesized through wet chemical and high temperature methods, followed by thorough characterization to examine the importance of material property changes in NP-induced toxicity. We found our diverse set of $\mathrm{nZnO}$ displayed significant differences in surface reactivity, dissolution potential and cytotoxicity towards cancerous and primary $\mathrm{T}$ cells. Additionally, principal component analysis (PCA) suggested that the synthesis procedure conferred unique material properties, and can be a determinant of cellular cytotoxicity. Furthermore, we showed that attributing NP-induced toxicity to one specific material property is shortsighted and that complex interactions between these properties needs to be considered.

Finally, Chapter 4 introduces future work dedicated to investigating transcriptome changes in cancerous and primary $\mathrm{T}$ cells exposed to $\mathrm{nZnO}$. Both cell types demonstrated significant up- and down-regulation of genes in a dose-dependent manner. Many significant differentially expressed genes (SDEGs) corresponded to proteins involved in 
the sequestration and transport of ionic zinc confirming the importance of $\mathrm{nZnO}$ in the cytotoxic response. Additional analysis will focus on the importance of specific SDEGs involved in the regulation of oxidative stress pathways, cellular metabolism, inflammation, T cell activation, and protein misfolding in the NP-induced toxicity mechanism. 


\section{TABLE OF CONTENTS}

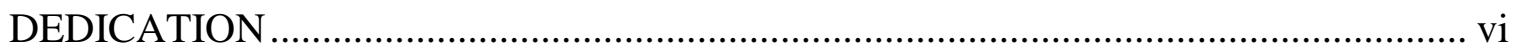

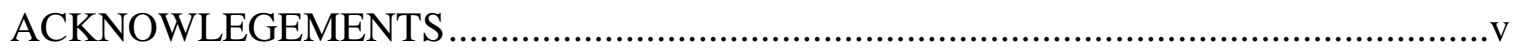

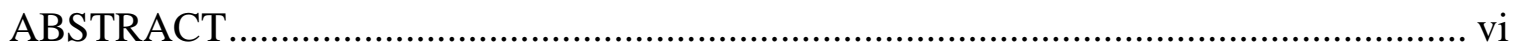

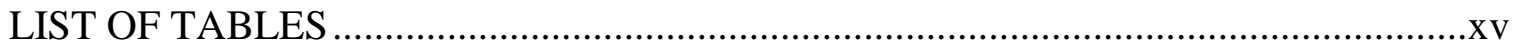

LIST OF FIGURES …................................................................................

LIST OF ABBREVIATIONS ........................................................................ xxvi

CHAPTER 1 INTRODUCTION ..................................................................

Zinc Oxide Nanoparticles - Cancer Therapeutic or Environmental Health Crisis?.1

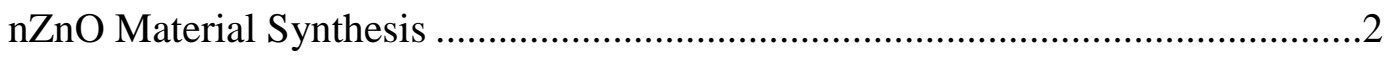

Electronic Structure and Surface Reactivity ..................................................

Intrinsic Surface Defects and Photocatalytic Activity .............................5

Zeta Potential .................................................................................

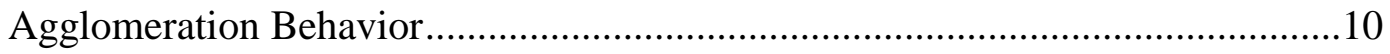

Colloidal Stability ................................................................. 10

Strategies to Achieve Dispersion Stability ..........................................12

Dissolution Potential ................................................................................... 14

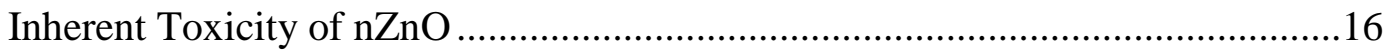


Surface Property Contributions to nZnO Toxicity and Oxidative Stress ..16

Dissolution Contributions to nZnO Toxicity and Oxidative Stress

Cellular Regulation of and Response to Zinc and $\mathrm{nZnO}$

Maintaining Zinc Homeostasis .20

Metallothioneins and Zinc Transporters ............................................21

Zinc Signaling and Influences on Zinc Homeostasis.............................23

ZnO Nanoparticles and Genetic Expression ..........................................25

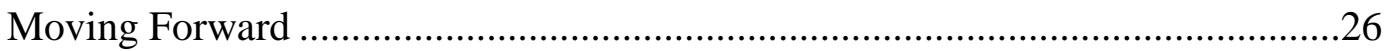

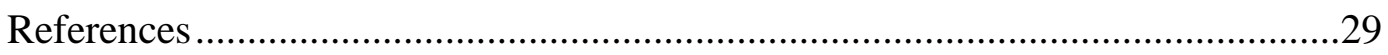

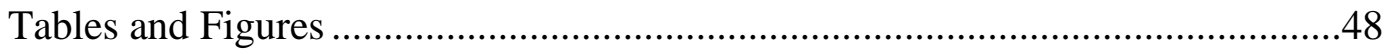

\section{CHAPTER 2 SERUM PROTEINS ENHANCE DISPERSION STABILITY AND INFLUENCE THE CYTOTOXICITY AND DOSIMETRY OF ZNO NANOPARTICLES IN SUSPENSION AND ADHERENT CANCER CELL MODELS 64}

Abstract .65

Keywords .66

Background .66

Methods .70

Synthesis and Characterization of $\mathrm{nZnO}$ .70

NP Stock Preparation .72

Extracellular Dissolution .73

Dosimetry Analysis and Empirical Deposition Fractions....... .74

Cell Culture and Cytotoxicity Studies .76

Reactive Oxygen Species (ROS) Detection.............................................78

Statistical Analyses .79 


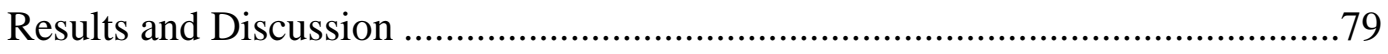

$\mathrm{ZnO}$ Nanoparticle Synthesis and Characterization .....................................79

Agglomeration and Sedimentation Profiles ..............................................81

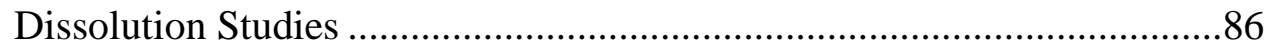

Reactive Oxygen Species (ROS) ………………………………….......8

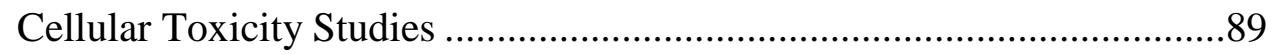

Modeling and Dosimetry Studies..................................................................93

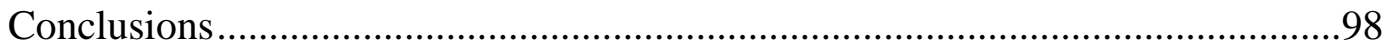

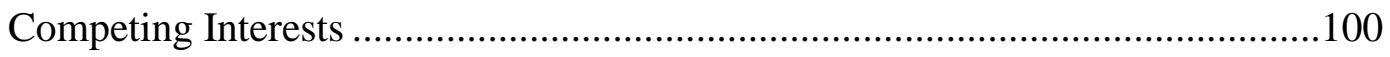

Authors' Contributions ……………………………....................................100

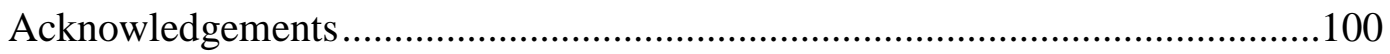

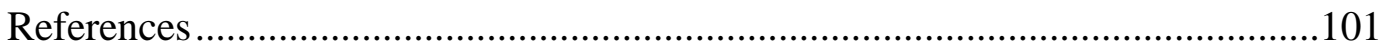

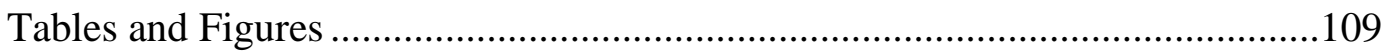

\section{CHAPTER 3 ZNO NANOPARTICLE PREPARATION ROUTE INFLUENCES} SURFACE REACTIVITY, DISSOLUTION AND CYTOTOXICITY ……...................124

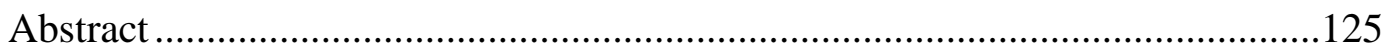

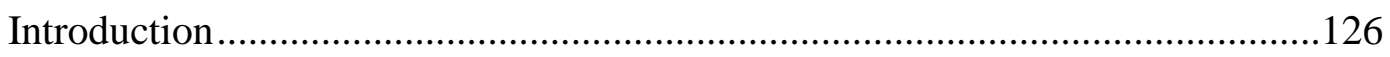

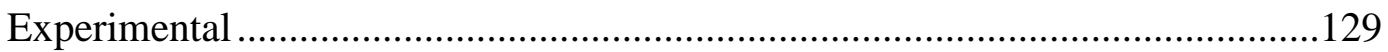

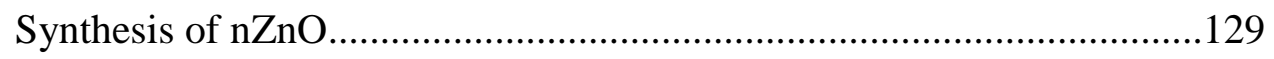

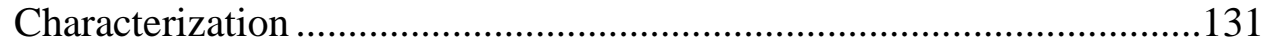

Cell culture and cytotoxicity studies........................................................132

Photocatalytic Studies ........................................................................133

Extracellular dissolution of nZnO stock solutions.....................................134

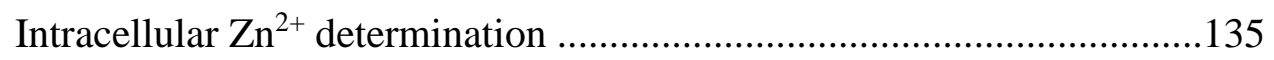


Cell-associated $\mathrm{Zn}^{2+}$

Dosimetry......

Zinc speciation determination

Reactive Oxygen Species (ROS) Detection

Statistical analyses

Results

X-ray Diffraction, XPS and TEM Analysis for Crystal Structure,

Composition, and Morphology

Cellular toxicity and oxidative stress responses

Surface characterization and reactivity

Dissolution potential and zinc speciation

Dosimetry......

Principal Component Analysis

Discussion

Conclusions

Conflicts of interest.

Acknowledgements.

References

Tables and Figures 166

Electronic Supplementary Information 178

XRD, XPS and TEM Images 178

Cellular Toxicity 178

Dissolution Kinetics for Nanopure Water Stock Solutions 178

FTIR and FTIR Peak Deconvolution 179 
Principal Component Analysis ...........................................................180

Tables and Figures - Electronic Supplementary Material ..................................181

References - Electronic Supplementary Information .........................................199

\section{CHAPTER 4 CELLULAR TRANSCRIPTOME RESPONSES IN JURKAT}

LEUKEMIC AND PRIMARY CD4 ${ }^{+} \mathrm{T}$ CELLS FOLLOWING ZNO NANOPARTICLE TREATMENT 201

Introduction .201

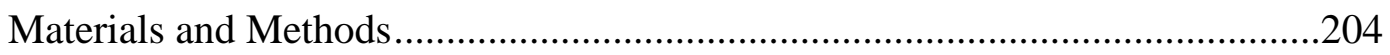

Zinc Oxide Synthesis and Characterization ...............................................204

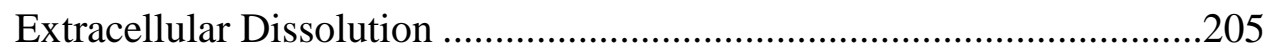

Cell Culture and Toxicity Experiments .....................................................206

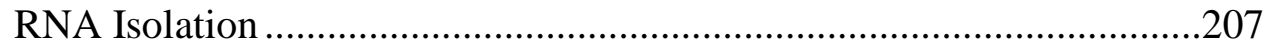

Microarray Analysis of Global Gene Expression Profile ........................208

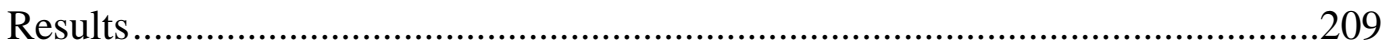

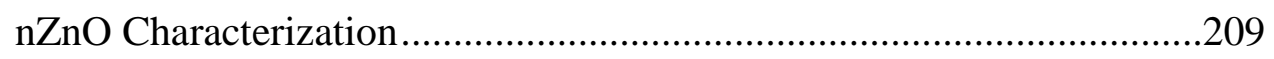

Effects of $\mathrm{nZnO}$ on Jurkat Leukemic and Primary $\mathrm{CD} 4^{+}$Cell

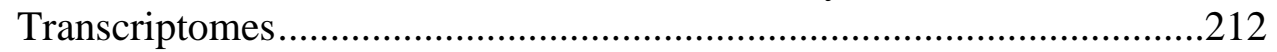

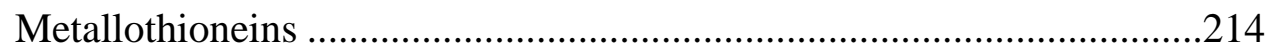

Solute-Linked Carrier Proteins ...............................................................215

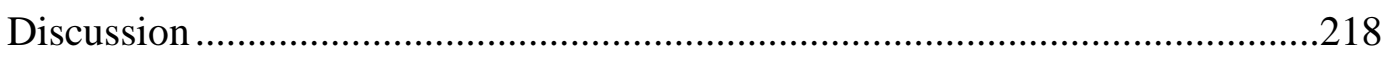

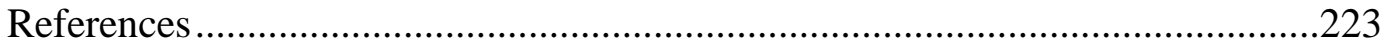

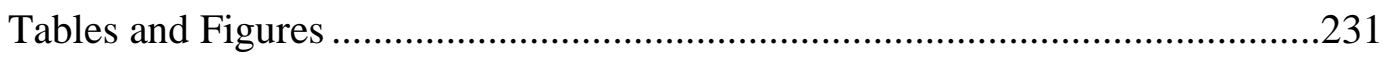

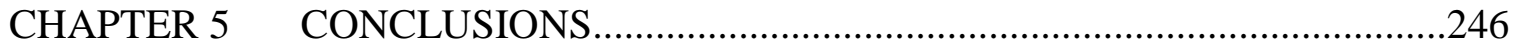


Nature Publishing Group License Terms and Conditions

Article: Role of Zinc Signaling In The Immune System .....

Article: Serum Proteins Enhance Dispersion Stability and Influence the Cytotoxicity and Dosimetry of $\mathrm{ZnO}$ Nanoparticles in Suspension and Adherent Cancer Cell Models..............................................................250

Article: ZnO Nanoparticle Preparation Route Influences Surface Reactivity, Dissolution and Cytotoxicity 


\section{LIST OF TABLES}

Table 1.1 Common liquid and gas phase chemical synthesis methods for $\mathrm{nZnO}$ including potential applications found in literature. .................................. 50

Table 2.1 Delivered Dose Metrics. ………………….................................... 123

Table 3.1 Physical properties of $\mathrm{ZnO}$ formulations measured via TEM, BET, UVVis, and DLS. Wet chemically and FSP synthesized formulations are shaded green and blue, respectively, while the control samples are indicated with the dark grey. The average size values are based on a minimum of 100 measured NPs on TEM images. The hydrodynamic diameters were determined by measuring the number of particles within each size bin in the histogram distribution.

Table 4.1 The number of SDEGs in each functional class. Functional classes were designated by Ingenuity Pathway Analysis (IPA) knowledge base. The yellow bars represented the $\mathrm{nZnO}$ treatment concentration closest to the $\mathrm{IC}_{50}$ for each cell type.

Table 4.2 Fold change values for selected genes relating to inflammation, $\mathrm{T}$ cell activation and cellular stress.

Table S3.1 The Jurkat and primary $\mathrm{CD}^{+}{ }^{+} \mathrm{T}$ cell $\mathrm{IC}_{50}$ values converted to millimolar $(\mathrm{mM})$ concentrations and the calculated therapeutic index.

Table S3.2 Linear regression model for $\mathrm{IC}_{50}$ as a linear function of PC1 and PC2.. 192

Table S3.3 Identified FTIR peaks for the 24-hour precipitate analysis after deconvolution of the broad $\mathrm{ZnO}$ band (350-750 $\left.\mathrm{cm}^{-1}\right)$ including the possible peak assignment and reference 


\section{LIST OF FIGURES}

Figure 1.1 Worldwide distribution of nZnO usage (data adapted from KołodziejczakRadzimska (2014). .............................................................................. 48

Figure 1.2 Schematic representation of the application of $\mathrm{nZnO}$........................... 49

Figure 1.3 Reaction steps in the formation of $\mathrm{nZnO}$ during flame spray pyrolysis... 51

Figure 1.4 The main photophysical processes of $\mathrm{nZnO}$ when excited by UV radiation where $\mathrm{h}_{v} \geq \mathrm{E}_{\mathrm{g}}$. The four processes represent are: I) photo-excited process (gray arrows), II) band -band photoluminescence process (green arrow), III) excitonic process (red arrow), and IV) non-radiative transition process (blue arrows). The empty spheres in the valance band (VB) represent valance band holes $(\mathbf{h v b}+)$ and solid spheres in the conduction band (CB) denote conduction band electrons (ecb -) 52

Figure 1.5 Sonocatalytic and photocatalysis reaction processes for $\mathrm{nZnO}$ including redox reactions, reactive intermediates, and reactive oxygen species..... 53

Figure 1.6 DLVO graph depicting colloidal stability for a NP dispersion................. 54

Figure 1.7 Schematic illustrating the transport dynamics of nanoparticles when introduced to adherent cell culture models in both the upright (left side) and inverted (right side) configurations. Cho article Figure 4. Reprinted by permission from Macmillan Publishers Ltd: Nature Nanotechnology (Cho, E. C.; Zhang, Q.; Xia, Y. The effect of sedimentation and diffusion on cellular uptake of gold nanoparticles. Nature Nanotechnology 2011, 6, 385-391.), copyright 2011.

Figure 1.8 Schematic illustrating dispersion dynamics within cell culture................ 56

Figure 1.9 Schematic depicting the dissolution processes associated with nZno. ..... 57

Figure 1.10 Schematic illustrating the various mechanisms by which (A) exogenous ROS generated at the $\mathrm{nZnO}$ surface and the $\mathrm{nZnO}$ particles themselves can induce cellular damage including (B) membrane disruption and lipid peroxidation. (C) Internalized ROS via membrane bound transporters will cause (D) oxidative damage to cellular components or activate stressinduced signaling. (E) Electrostatic interactions of NPs with cellular 
membranes will facilitate NP adsorption and cellular membrane damage.

Figure 1.11 Schematic illustrating the various mechanisms by which extracellular, intracellular free, and cell-associated zinc induce cellular damage. Extracellular free zinc can enter cells through (A) non-specific ion channels, and (B) specialized zinc importers (Zips). Intact NPs or amorphous complexes may be internalized through (C) active or (D) passive transport mechanisms. Elevated intracellular free zinc concentrations are remediated through sequestration into (E) zincosomes or by $(\mathrm{F})$ metallothioneins. Disruption of zinc homeostasis can result in (G) mitochondrial and organelle damage or $(\mathrm{H})$ genotoxicity.

Figure 1.12 The number of human genes encoding for proteins with a zinc-binding site.

Figure 1.13 Schematic illustrating the cellular localization of the SLC39 (Zips) and SLC30 (ZnTs) families of zinc transporters. Zip proteins (blue) elevate cytosolic zinc levels by importing ionic zinc from the extracellular environment or cellular compartments. ZnT transporters lower cytosolic zinc through efflux into cellular compartments or out of the cell. Metallothioneins contribute to intracellular zinc level management by sequestering zinc ions. Hojya and Fukada article Figure 2. Reprinted by permission from Hindawi Publishers: Journal of Immunology Research (Shintaro Hojyo and Toshiyuki Fukada, "Roles of Zinc Signaling in the Immune System," Journal of Immunology Research, vol. 2016, Article ID 6762343, 21 pages, 2016. doi:10.1155/2016/6762343), copyright 2016. 61

Figure 1.14 Early (A) and late (B) zinc signaling. 62

Figure 1.15 Schematic overview summarizing the toxic effect of nZnO. The key material factors implicated in NP-induced toxicity include particle size and shape, agglomeration potential, surface structure and reactivity, and NP dissolution. The observed cellular responses include disruption of zinc homeostasis, metallothionein and zinc transport regulation, zinc related cell signaling, and oxidative stress

Figure 2.1 Crystal phase composition, size and morphology characterization for $\mathrm{nZnO}$ and $\mathrm{nZnO}-\mathrm{FBS}^{\text {dried }}$ NPs. A) XRD spectra and TEM images for B) $\mathrm{nZnO}$ and $\mathrm{C}) \mathrm{nZnO}-\mathrm{FBS}^{\text {dried }}$.

Figure 2.2 Surface property analysis for $\mathrm{nZnO}$ and $\mathrm{nZnO}-\mathrm{FBS}^{\text {dried }}$. A) Zeta potentials measured as a function of $\mathrm{pH}$ and B) FTIR spectra with the wavenumbers of the main peaks marked. 
Figure 2.3 Aggregation profile evaluation for NP stock solutions. Average hydrodynamic size profiles of $25 \mathrm{mM} \mathrm{ZnO}$ stock dispersions $(\mathrm{pH}=7.3$ ) for a 24-hour time period. A) $\mathrm{nZnO}$ in various biologically relevant solutions. B) $\mathrm{nZnO}, \mathrm{nZnO}-\mathrm{FBS}^{\text {dried }}, \mathrm{nZnO}-\mathrm{FBS}^{\text {washed }}$ and $\mathrm{nZnO}-\mathrm{FBS}$ stock dispersions in $\mathrm{PBS}$ and $\mathrm{C}$ ) $\mathrm{nZnO}, \mathrm{nZnO}-\mathrm{FBS}^{\text {dried }}, \mathrm{nZnO}-$ $\mathrm{FBS}^{\text {washed }}$ and $\mathrm{nZnO}-\mathrm{FBS}$ stock dispersions in nanopure water.

Figure 2.4 Sedimentation profile evaluation for NP stock dispersions. Sedimentation profiles of $25 \mathrm{mM} \mathrm{ZnO}$ stock dispersions $(\mathrm{pH}=7.3$ ) for a 2-hour time period. A) $\mathrm{nZnO}, \mathrm{nZnO}-\mathrm{FBS}^{\text {dried }}, \mathrm{nZnO}-\mathrm{FBS}^{\text {washed }}$ and $\mathrm{nZnO}-\mathrm{FBS}$ stock dispersions in various biologically relevant solutions. B)

Sedimentation histograms for $0,0.5,1.0,1.5$ and 2.0 hour time points for $\mathrm{nZnO}-\mathrm{FBS}^{\text {dried }}, \mathrm{nZnO}-\mathrm{FBS}^{\text {washed }}$ and $\mathrm{nZnO}-\mathrm{FBS}$ stock dispersions in PBS and nanopure water. 112

Figure 2.5 Hydrodynamic size distribution profiles at 0, 12 and 24 hours. Histograms represent $25 \mathrm{mM}$ stock dispersions prepared in A) nanopure water B) PBS and C) RPMI-based cellular media. 113

Figure 2.6 Hydrodynamic size distribution profiles at 0, 12 and 24 hours. Histograms represent $25 \mathrm{mM}$ stock dispersions prepared in PBS for A) $\mathrm{nZnO}-$ $\left.\mathrm{FBS}^{\text {dried }}, \mathrm{B}\right) \mathrm{nZnO}-\mathrm{FBS}^{\text {washed }}$ and C) $\mathrm{nZnO}-\mathrm{FBS}$. 114

Figure 2.7 Hydrodynamic size distribution profiles at 0, 12 and 24 hours. Histograms represent $25 \mathrm{mM}$ stock dispersions prepared in water for $\mathrm{A}$ ) $\mathrm{nZnO}$ $\mathrm{FBS}^{\text {dried }}$, B) $\mathrm{nZnO}-\mathrm{FBS}^{\text {washed }}$ and C) $\mathrm{nZnO}-\mathrm{FBS}$. 115

Figure 2.8 Dispersion stability analysis for nZnO/PBS and nZnO - FBS/PBS stock solutions introduced to RPMI-based cellular media at a concentration of $0.6 \mathrm{mM}$. A) Average hydrodynamic size profiles for a 24-hour time period. B) Sedimentation profiles for a 24-hour time period.

Hydrodynamic size distribution profiles at 0, 12 and 24 hours for $\mathrm{C}$ ) nZnO/PBS stock dispersion in RPMI-based cellular media and D) nZnO FBS/PBS stock dispersion in RPMI-based cellular media. 116

Figure 2.9 Extracellular dissolution of $6 \mathrm{mM}$ nZnO/PBS and nZnO - FBS/PBS stock dispersions and $0.6 \mathrm{mM}$ of those respective $\mathrm{nZnO}$ stock dispersions in RPMI-based cellular media. $\mathrm{Zn}^{2+}$ concentration is expressed as $\% \mathrm{Zn}^{2+}$ (total dissolved $\mathrm{Zn}^{2+}$ measured by ICP-MS / total $\mathrm{Zn}^{2+}$ in sample) to normalize for concentration.

Figure 2.10 Cellular production of ROS following ZnO NP exposure. ROS generation was evaluated in Jurkat cells at 6,18 and 24 hours following treatment with $\mathrm{nZnO} / \mathrm{PBS}$ (PBS) and nZnO - FBS/PBS (FBS/PBS) stock dispersions using MitoSOX red and flow cytometry (means \pm standard error, minimum of $\mathrm{n}=3$ ). Cells were treated with $0.4 \mathrm{mM} \mathrm{nZnO}$ and stained 
with MitoSOX red and CD3 antibody after the desired time. A) Mean MitoSOX fluorescence intensity for a non-treated control (NT) and $\mathrm{nZnO}$ and $\mathrm{nZnO}-\mathrm{FBS}$ exposure. To assess relative increases in ROS following NP treatment, a marker (M1) was set so that background fluorescent in control samples (MitoSOX loaded/no NP) was between 1 and $3.5 \%$. Histograms represent B) 6 hours, C) 18 hours and D) 24 hours. 118

Figure 2.11 ZnO NP toxicity using nZnO/PBS and nZnO-FBS/PBS stock dispersions for 24 hours on various suspension cell types. Cultures were treated concurrently with varying concentrations of $\mathrm{ZnO} \mathrm{NP}$ for 24 hours and cell viability was evaluated (means \pm standard error, minimum of $n=3$ ). Statistical analysis was performed using repeated measures analysis of variance and model-based means post hoc test $(\mathrm{p}<0.05)$ with an asterisk denoting statistical significance. Jurkat cell viability was assessed using both (A) alamar blue staining or (B) flow cytometry and PI uptake to evaluate between assay variability and repeatability. (C) Hut-78 cell viability using alamar blue staining and (D) $\mathrm{CD} 4^{+} \mathrm{T}$ Cell viability using flow cytometry and PI uptake. 119

Figure 2.12 $\mathrm{ZnO} \mathrm{NP}$ toxicity using varying concentrations of $\mathrm{nZnO} / \mathrm{PBS}$ and $\mathrm{nZnO}$ FBS/PBS stock dispersions for 24 hours on adherent cell types. (A) T-47D epithelial mammary gland carcinoma and (B) $\mathrm{LNCaP}$ epithelial prostate cancer cells. Cultures were treated concurrently and cell viability was evaluated (means \pm standard error, minimum of $n=3$ ). Statistical analysis was performed using repeated measures analysis of variance and modelbased means post hoc test $(\mathrm{p}<0.05)$ with an asterisk denoting statistical significance.

Figure 2.13 Dosimetry curves for various stock dispersions introduced to RPMI-based cellular media at a concentration of $1.25 \mathrm{mM}$. A) Curves constructed using $\alpha$ values calculated for 24-well plates and representing the fraction of NP deposited $\left[f_{D}(t)\right]$ to an adherent cell as a function of time. B) Curves constructed using $\alpha$ values calculated for 96-well plates and representing the fraction of NPs available $\left[f_{A}(t)\right]$ to cells grown in suspension as a function of time. Dosimetry curves constructed using both the Harvard VCM and ISDD models for both the nZnO/PBS and nZnO - FBS/PBS stocks representing C) NP deposition $\left[f_{D}(t)\right]$ and D) NP availability $\left[f_{A}(t)\right]$.

Figure 2.14 Dosimetry curves constructed using both the Harvard VCM and ISDD models for $\mathrm{nZnO} / \mathrm{PBS}$ and $\mathrm{nZnO}$ - FBS/PBS stock dispersions introduced to RPMI-based cellular media at a concentration of $1.25 \mathrm{mM}$. Curves were constructed using $\alpha$ values calculated for 24-well plates at various time points (ISDD) and Harvard VCM $\alpha$ values (Table 1). These curves represent the fraction of NPs deposited $\left[f_{D}(t)\right]$ to an adherent cell layer as a 
function of time for A) $\mathrm{nZnO}$ - PBS stock dispersions and B) $\mathrm{nZnO}-$ FBS/PBS stock dispersions.

Figure 3.1 NP-induced toxicity values for (a) Jurkat leukemic cell and (b) normal primary $\mathrm{CD}^{+} \mathrm{T}$ cell viability at 24 hours after treatment with the wet chemical method (green bars; wet), flame spray pyrolysis (blue bars; FSP) $\mathrm{nZnO}$ formulations and the $\mathrm{SiO}_{2}$-FSPR and bulk controls (black bars; control). The white labels on the histogram bars depict the $\mathrm{IC}_{50}$ values obtained for the indicated sample. The histogram bars were ordered from lowest to highest $\mathrm{IC}_{50}$ for both cell types to depict the synthesis method trends observed for the NP-induced toxicity. Cultures were treated concurrently with varying concentrations of $\mathrm{nZnO}$ dispersed in nanopure water/RPMI for 24 hours and cell viability was evaluated (means \pm standard error, minimum of $\mathrm{n}=3$ ) using Alamar blue staining (Jurkat Cells) or flow cytometry with PI staining $\left(\mathrm{CD}^{+}{ }^{+} \mathrm{T}\right.$ cells $)$. Statistical analysis was performed using repeated measures analysis of variance and model-based means post hoc test $(\mathrm{p}<0.05)$ with differing letters denoting statistical significance. Linear contrast models were used to determine statistical significance between the wet chemical, flame spray pyrolysis and control samples.

Figure 3.2 nZnO samples generated ROS in a formulation-dependent manner. Mitochondrial superoxide generation by all $\mathrm{nZnO}$ at 24-hour post treatment with $32.4 \mu \mathrm{g} / \mathrm{mL} \mathrm{ZnO}$ using flow cytometry and MitoSox ${ }^{\mathrm{TM}}$ Red staining. Statistical analysis was performed using repeated measures analysis of variance and model-based means post hoc test $(\mathrm{p}<0.05)$ with differing letters denoting statistical significance. 170

Figure 3.3 Surface property characterization for the powered samples ((a) and (b)). and catalytic activity plots ((c) and (d)) depicting the UV/Vis monitored fluorescence of the model sulfo-Rhodamine B dye and the average dye degradation kinetic values obtained for the evaluated treatment conditions. FTIR spectra for (a) wet chemical synthesis methods, and (b) heat treatment methods illustrate the peaks observed and the corresponding wavenumber values. The graph in (c) is representative of the timedependent plots obtained and demonstrate the photocatalytic decomposition of sulfo-Rhodamine B dye in nanopure water after treatment with EG NPs. The histogram in (d) depicts the average catalytic rate constants $\left(\mathrm{k}, \mathrm{min}^{-1}\right)$ for the $\mathrm{nZnO}$ and control samples. Statistical analysis in (d) was performed using repeated measures analysis of variance and model-based means post hoc test $(\mathrm{p}<0.05)$ with differing letters denoting statistical significance. Histogram bars represent $n=3$ replicates with error bars indicating s.e.

Figure 3.4 $\quad \mathrm{nZnO}$ formulations display similar dissolution kinetic trends in cellular media at 4 and 24 hours. Extracellular (EC) $\mathrm{Zn}^{2+}$ concentrations $(\mu \mathrm{g} / \mathrm{mL})$ 
measured via ICP-MS (bars and left y-axis) and intracellular $\mathrm{Zn}^{2+}$ concentrations measured via flow cytometry and expressed as mean fluorescence intensity (MFI) of the zinc specific dye FluoZin-3 AM (line graphs and right y-axis) evaluated at 4 hours (top graph) and 24 hours (bottom graph). Control samples, designated as the left most bar or line graph symbol were RPMI-based cellular media (EC assay) and NT cells (Intracellular (IC) assay). Statistical analysis was performed for the extracellular zinc measurements (histogram bars) using repeated measures analysis of variance and model-based means post hoc test $(\mathrm{p}<0.05)$ with differing letters denoting statistical significance. Histogram bars or line graph circles represent the average of $n=4$ replicates with error bars depicting s.e.

Figure 3.5 Cell-associated (CA) $\mathrm{Zn}^{2+}$ concentrations $(\mu \mathrm{g} / \mathrm{L})$ measured at 4 and 24 hours via ICP-MS. A control sample designated as the left most grey bar, were non-treated cells grown in cellular media. Statistical analysis was performed using repeated measures analysis of variance and model-based means post hoc test $(\mathrm{p}<0.05)$ with differing letters denoting statistical significance. Histogram bars represent the average of $n=4$ replicates with s.e. error bars

Figure 3.6 Representative samples highlighting the FTIR region from 1800 to 350 $\mathrm{cm}^{-1}$ and the $\mathrm{ZnO}$ peak deconvolution. The figure includes (a) FTIR spectra from 1800 to $350 \mathrm{~cm}^{-1}$ for the EG nZnO sample at 4 hours (blue) and 24 hours (red) and peak deconvolution of the broad FTIR band from $750-350 \mathrm{~cm}^{-1}$ for the EG nZnO sample at (b) 4-hour and (c) 24-hour time points. Samples were introduced to cellular media at a concentration of 32 $\mu \mathrm{g} / \mathrm{mL}$ and incubated for the indicated time points. After incubation, the dispersions were centrifuged and the precipitate retained and dried overnight at $60^{\circ} \mathrm{C}$.

Figure 3.7 The integrated area ratio of $\mathrm{ZnO}$ to PO43- from the FTIR spectra and the atomic concentration ratio of $\mathrm{Zn} / \mathrm{P}$ from the XPS survey spectra of the insoluble zinc amorphous precipitates isolated from $\mathrm{nZnO}$ dispersions in cellular media post incubated for 4 and 24 hours. The left-hand side of the figure represents the integrated area ratio of $\mathrm{ZnO}$ to $\mathrm{PO}_{4}{ }^{3-}$ from the FTIR spectra (line graphs with circles and first right $y$-axis) and the atomic concentration ratio of $\mathrm{Zn} / \mathrm{P}$ from the XPS survey spectra (line graphs with tringles and second right y-axis) evaluated at (a) 4 hours and (b) 24 hours. The faded histogram bars represented the CA zinc results presented in Fig. 3.5 and are included for reference. The right-hand side of the figure represents the XPS spectra from $1027 \mathrm{eV}$ to $1018.5 \mathrm{eV}$ illustrating the deconvolution of the $\mathrm{Zn} 2 \mathrm{p}_{3 / 2}$ peak for the (c) EG $\mathrm{nZnO}$ as prepared sample and the (d) EG $\mathrm{nZnO}$ in cellular media at the 4-hour time point........... 175 
Figure 3.8 The time-dependent deposition nZnO onto the bottom of a well in a 96well culture plate as determined by ISDD dosimetry modelling for the individual $\mathrm{nZnO}$ formulations. The total amount of $\mathrm{nZnO}$ introduced to the cellular media was $6.48 \mu \mathrm{g}(32.4 \mu \mathrm{g} / \mathrm{mL}$ introduced into $0.2 \mathrm{~mL}$ of RPMI-based cellular media). The curves in (a) represent the calculated $\mathrm{nZnO}$ deposition over a 30-hour period. The histogram bars (left y-axis) in (b) demonstrate the modelled concentration of $\mathrm{nZnO}$ deposited at the 24hour time point with the half white/half black circles (firs right y-axis) correlating to hydrodynamic size and the red triangles (second right yaxis) representing the specific surface area (SSA) as measured by BET. Error bars in (b) represent s.e. with $n=3$ replicates........................... 176

Figure 3.9 PCA model-generated values for both PC1 and PC2 and a graphical representation of the PC scores and Jurkat cells $\mathrm{IC}_{50}$ values for each evaluated sample. The table in (a) represents the loading values (x 100) for each measured variable, eigenvalues and the percent variation explained for each PC. The indicated abbreviations refer to hydrodynamic size (hydrosize), intracellular zinc $\left[\mathrm{IC} \mathrm{Zn}^{2+}\right]$, cell-associated-zinc $\left[\mathrm{CA} \mathrm{Zn}^{2+}\right]$, and extracellular zinc $\left[\mathrm{EC} \mathrm{Zn}^{2+}\right]$ concentrations. The green cubes (wet chemical methods), blue spheres (FSP method) and black tetrahedrons (controls samples) depicted in the 3D graph in (b) represent PC1 scores versus $\mathrm{IC}_{50}$ values versus PC2 scores. The colored ellipses denote sample grouping based on similar PC1 and/or PC2 scores.

Figure 4.1 Size, morphology and composition analysis for the $\mathrm{nZnO}$. A) represents the XRD spectrum used to verify crystal structure and phase purity. TEM analysis (B) was employed to confirm NP morphology and average particle size. The atomic composition of the powered nanomaterial was determined using XPS (C)

Figure 4.2 Surface structure analysis of the nZnO. A) Surface adsorbed groups were evaluated using FTIR and B) $\zeta$-potential and IEP were determined using DLS.

Figure 4.3 The A) agglomeration tendencies and B) dissolution potential of $\mathrm{nZnO}$ dispersions were evaluated utilizing DLS measurements and ICP-MS, respectively. The $\mathrm{nZnO}$ dispersions utilized for both assays were created by introducing a nZnO FBS/PBS stock solution introduced to RPMI-based cellular media at a concentration of $486 \mu \mathrm{g} / \mathrm{mL}$. Error bars in (B) represent the standard deviation of $n=3$ replicates. 233

Figure 4.4 n $\mathrm{nZnO}$ induced toxicity in Jurkat leukemic (green squares and line) and primary $\mathrm{CD}^{+} \mathrm{T}$ cells (blue circles and line) measured at A) 6 hours and B) 24 hours post treatment. The dashed lines in both plots represent the mathematical fit for the plotted data points. The bottom axis represents $\mathrm{nZnO}$ concentrations for Jurkat cells and the top axis depicts $\mathrm{nZnO}$ 
concentrations for the $\mathrm{CD} 4^{+} \mathrm{T}$ cells. C) The number of significantly differentially expressed genes for Jurkat leukemic (green patterned bars) and primary $\mathrm{CD}^{+} \mathrm{T}$ cells (blue patterned bars) determined using cutoff values of (fold change) FC $>1.5$ and (false discovery rate) FDR $\mathrm{p}<0.05$. D) The number of up- and down-regulated differentially expressed genes for Jurkat (green patterned bars) and primary $\mathrm{CD}^{+} \mathrm{T}$ cells (blue patterned bars). Up-regulated genes are represented as positive values and downregulated genes as negative values.

Figure 4.5 Differential expression of metallothionein genes in Jurkat and primary $\mathrm{CD}^{+} \mathrm{T}$ cells treated with varying concentrations of $\mathrm{nZnO}$ for 6 hours. All displayed genes statistically significant $(\mathrm{FC}>1.5$ and FDR $\mathrm{p}<0.05)$ and represent the average of four replicates. 236

Figure 4.6 The expression of genes from the $S L C 30, S L C 39, S L C 31, S L C 11$ and $S L C 40$ transporter families in Jurkat and primary $\mathrm{CD}^{+} \mathrm{T}$ cells treated with varying concentrations of $\mathrm{nZnO}$ for 6 hours. All displayed genes statistically significant $(\mathrm{FC}>1.5$ and FDR $\mathrm{p}<0.05)$ and represent the average of four replicates.

Figure 4.7 The expression of genes from the $S L C 2 A$, SLC45A, and SLC50A transporter families in Jurkat and primary $\mathrm{CD} 4^{+} \mathrm{T}$ cells treated with varying concentrations of $\mathrm{nZnO}$ for 6 hours. All displayed genes statistically significant $(\mathrm{FC}>1.5$ and FDR $\mathrm{p}<0.05$ ) and represent the average of four replicates....

Figure 4.8 The expression of genes from the SLC1A, SLC3A, SLC7A, and SLC15A transporter families in Jurkat and primary $\mathrm{CD} 4^{+} \mathrm{T}$ cells treated with varying concentrations of $\mathrm{nZnO}$ for 6 hours. All displayed genes statistically significant $(\mathrm{FC}>1.5$ and FDR $\mathrm{p}<0.05)$ and represent the average of four replicates

Figure S3.1 Crystal phase composition, size and morphology characterization for nZnO NPs. XRD spectra for (a) $\mathrm{nZnO}$ synthesized through wet chemical synthesis methods and (b) nZnO synthesized through flame spray pyrolysis (FSP) synthesis methods. TEM images for (c) DEG, (d) EG (e) $\mathrm{EtOH}$, (f) $\mathrm{NaOH}$, (g) FSPS, (h) FSPR, (i) $\mathrm{SiO}_{2}$-FSPR and (j) bulk samples were used to identify morphology and average NP size and distribution.

Figure S3.2 XPS survey scans for all tested powder samples .................................... 183 
Figure S3.3 $\mathrm{IC}_{50}(\mu \mathrm{g} / \mathrm{mL})$ values plotted as a function of the $\mathrm{nZnO}$ zeta potential $(\mathrm{mV})$ in aqueous conditions depicting the linear correlation between the two measures variables for wet chemical and FSP synthesized nZnO. 184

Figure S3.4 Dissolution kinetics for nanopure water dispersions for all tested formulations 185

Figure S3.5 FTIR spectra obtained for the amorphous precipitate material retained at the 4-hour [(a) and (c)] and 24-hour [(b) and (d)] time points. Samples were introduced to cellular media at a concentration of $32 \mu \mathrm{g} / \mathrm{mL}$ and incubated for the indicated time points. After incubation, the dispersions were centrifuged and the precipitate retained and dried overnight at $60{ }^{\circ} \mathrm{C}$.

Figure S3.6 FTIR peak deconvolution results for all powdered samples post incubation in cellular media for 24 hours. Each graph is labeled with the sample name with legend numbers corresponding to the peak position (in $\mathrm{nm}$ ) for each identified peak during the deconvolution process. 187

Figure S3.7 XPS survey scans for all tested samples incubated in cellular media for (a) 4 hours and (b) 24 hours. 188

Figure S3.8 Low (a) and (c) and high (b) and (d) resolution TEM images for the DEG sample precipitates isolated from RPMI-based cellular media at (a) and (b) 4-hour and (c) and (d) 24-hour time points.

Figure S3.9 Low (a) and (c) and high (b) and (d) resolution TEM images for the EG sample precipitates isolated from RPMI-based cellular media at (a) and (b) 4-hour and (c) and (d) 24-hour time points 190

Figure S3.10 Low (a) and (c) and high (b) and (d) resolution TEM images for the EtOH sample precipitates isolated from RPMI-based cellular media at (a) and (b) 4-hour and (c) and (d) 24-hour time points.

Figure S3.11 Low (a) and (c) and high (b) and (d) resolution TEM images for the $\mathrm{NaOH}$ sample precipitates isolated from RPMI-based cellular media at (a) and (b) 4-hour and (c) and (d) 24-hour time points.

Figure S3.12 Low (a) and (c) and high (b) and (d) resolution TEM images for the FSPS sample precipitates isolated from RPMI-based cellular media at (a) and (b) 4-hour and (c) and (d) 24-hour time points. 193

Figure S3.13 Low (a) and (c) and high (b) and (d) resolution TEM images for the FSPR sample precipitates isolated from RPMI-based cellular media at (a) and (b) 4-hour and (c) and (d) 24-hour time points. 194 
Figure S3.14 Low (a) and (c) and high (b) and (d) resolution TEM images for the $\mathrm{SiO}_{2}-$ FSPR sample precipitates isolated from RPMI-based cellular media at (a) and (b) 4-hour and (c) and (d) 24-hour time points.

Figure S3.15 Low (a) and (c) and high (b) and (d) resolution TEM images for the Bulk sample precipitates isolated from RPMI-based cellular media at (a) and (b) 4-hour and (c) and (d) 24-hour time points. 196 


\section{LIST OF ABBREVIATIONS}

ATCC

ANOVA

ATP

AuNP

BET

BSA

CA

CB

CD

CDKN1A

$\mathrm{Cu} / \mathrm{ZnSOD} 1$

Cys

$\mathrm{Cys}_{2}$

DC

DEG

DF

DLS

DLVO

DMEM

$\mathrm{DSE}_{\mathrm{cr}}$
American Type Culture Collection

Analysis of variance

Adenosine triphosphate

Gold nanoparticles

Brunauer-Emmett-Teller

Bovine serum albumin

Cell-associated

Conduction band

Cluster of differentiation

Cyclin-dependent kinase inhibitor 1

$\mathrm{Cu} / \mathrm{Zn}$ superoxide dismutase 1

Cysteine

Cystine

Dendritic cells

Diethylene-glycol

Fractal dimension

Dynamic light scattering

Derjaguin, Landau, Verwey, and Overbeek,

Dulbecco's Modified Eagle's medium

Critical material specific delivered sonication energy 


\begin{tabular}{ll} 
DTPA & Diethylenetriaminepentaacetic acid \\
EC & Extracellular \\
$\mathrm{e}_{\mathrm{cb}}^{-}$ & Conduction band electron \\
EDTA & Ethylenediaminetetraacetic acid \\
EG & Ethylene glycol \\
Eg & Electronic band gap \\
EPR & Enhanced permeability and retention effect \\
ESI & Electronic supplementary information \\
EtOH & Ethanol \\
FACS & Fluorescence assisted cell sorting \\
FBS & Fetal bovine serum \\
FC & Fold change \\
FceRI & FC potion high-affinity IgE receptor \\
FDR p & False discovery rate p-value \\
FITC & Fluorescein isothiocyanate \\
FSC & Forward scatter \\
FSP & Flame spray pyrolysis \\
FSPR & Flame spray pyrolysis rod-shaped \\
FSPS & Fourier transformed infrared spectroscopy \\
FTIR & Gastrointestinal \\
G.I. & GSH \\
GSH & Flutathione \\
\hline &
\end{tabular}




\begin{tabular}{|c|c|}
\hline Harvard VCM & Harvard volume computation method \\
\hline HMDSO & Hexamethyldisiloxane \\
\hline $\mathrm{H}_{2} \mathrm{O}_{2}$ & Hydrogen peroxide \\
\hline$h_{\mathrm{vb}}^{+}$ & Valence band holes \\
\hline $\mathrm{IC}_{25}$ & $25 \%$ inhibitory concentration \\
\hline $\mathrm{IC}_{50}$ & $50 \%$ inhibitory concentration \\
\hline ICP-MS & Inductively-coupled plasma mass spectrometer \\
\hline IEP & Isoelectric point \\
\hline IFN & Interferon \\
\hline $\operatorname{IgE}$ & Immunoglobulin E \\
\hline IL & Interleukin \\
\hline IPA & Ingenuity Pathway Analysis \\
\hline ISDD & In vitro sedimentation, diffusion and dosimetry \\
\hline LPS & Lipopolysaccharides \\
\hline MAPKK & Mitogen-activated protein kinase kinase \\
\hline MAUD & Materials Analysis Using Diffraction \\
\hline $\mathrm{M}-\mathrm{Cl}$ & Metal chloride \\
\hline MDA & Malondialdehyde \\
\hline MFI & MitoSox fluorescence intensity \\
\hline MHC & Major histocompatibility complex \\
\hline $\mathrm{M}-\mathrm{OOCCH}_{3}$ & Metal acetate \\
\hline $\mathrm{M}-\mathrm{OR}$ & Metal alkoxide \\
\hline MRE & Metal responsive element \\
\hline
\end{tabular}




\begin{tabular}{|c|c|}
\hline MT & Metallothionein \\
\hline MTF-1 & Metal-responsive transcription factor -1 \\
\hline MyD88 & Myeloid differentiation factor 88 \\
\hline NADPH & Nicotinamide adenine dinucleotide phosphate \\
\hline $\mathrm{NaOH}$ & Sodium hydroxide \\
\hline $\mathrm{NIOSH}$ & National Institute for Occupational Safety and Health \\
\hline NP & Nanoparticle \\
\hline $\mathrm{nZnO}$ & Nano zinc oxide \\
\hline $\mathrm{nZnO}-\mathrm{FBS}$ & FBS-coated nano $\mathrm{ZnO}$ \\
\hline $\mathrm{nZnO}-\mathrm{FBS}^{\text {dried }}$ & dried FBS-coated $\mathrm{nZnO}$ \\
\hline $\mathrm{nZnO}-\mathrm{FBS}^{\text {washed }} / \mathrm{PBS}$ & FBS-coated nano $\mathrm{ZnO}$ washed in PBS \\
\hline $\mathrm{nZnO}-\mathrm{FBS}^{\text {washed} / w a t e r}$ & FBS-coated nano $\mathrm{ZnO}$ washed in water \\
\hline $\mathrm{nZnO}-\mathrm{FBS} / \mathrm{PBS}$ & FBS-stabilized PBS nZnO dispersion \\
\hline $\mathrm{nZnO} / \mathrm{PBS}$ & PBS nZnO dispersion \\
\hline $\mathrm{O}_{2}$ & Diatomic oxygen \\
\hline$\cdot \mathrm{O}_{2}^{-}$ & Superoxide anion \\
\hline $\mathrm{OH}^{\cdot}$ & Hydroxyl radical \\
\hline $\mathrm{O}_{\mathrm{i}}$ & Neutral oxygen interstitial atom \\
\hline $\mathrm{O}_{\mathrm{i}}^{\prime}$ & Interstitial oxygen anions with one electron \\
\hline $\mathrm{O}_{\mathrm{i}}^{\prime \prime}$ & Interstitial oxygen anions with two electrons \\
\hline$\cdot \mathrm{OOH}$ & Perhydroxyl radical \\
\hline PAA & Poly (acrylic acid) \\
\hline PEG & Polyethylene glycol \\
\hline
\end{tabular}




\begin{tabular}{|c|c|}
\hline PBMC & Peripheral blood mononuclear cells \\
\hline PBS & Phosphate buffered saline \\
\hline $\mathrm{PC}$ & Principal component \\
\hline PCA & Principal component analysis \\
\hline PI & Propidium iodide \\
\hline PVP & Polyvinylpyrrolidone \\
\hline RIN & RNA integrity number \\
\hline ROS & Reactive oxygen species \\
\hline RPMI & Roswell Park Memorial Institute medium \\
\hline RT-PCR & Reverse transcription polymerase chain reaction \\
\hline SDEG & Significantly differentially expressed gene \\
\hline $\mathrm{SiO}_{2}$ & Silica or Silicon dioxide \\
\hline $\mathrm{SiO}_{2}$-FSPR & Silica coated FSP rod-shaped NPs \\
\hline SLC & Solute-linked carrier \\
\hline SOD & superoxide dismutase \\
\hline SSC & Side scatter \\
\hline TAE & Tris base, acetic acid and EDTA \\
\hline TEM & Transmission electron microscopy \\
\hline TIR & Toll/IL-1 receptor \\
\hline TLC & Toll-like receptor \\
\hline TOPO & Trinoctylphosphine oxide \\
\hline TPEN & $N, N, N^{\prime}, N^{\prime}$-tetrakis(2-pyridinylmethyl)-1,2- \\
\hline
\end{tabular}




\begin{tabular}{|c|c|}
\hline ТPP & Techno Plastic Products \\
\hline TRIF & TIR domain-containing adapter inducing interferon $\beta$ \\
\hline UV-A & Ultraviolet A \\
\hline UV-B & Ultraviolet B \\
\hline UV-Vis & Ultraviolet-visible \\
\hline $\mathrm{V}_{\mathrm{A}}$ & Attractive force \\
\hline VB & Valence band \\
\hline$V_{D}$ & Diffusion velocity \\
\hline$v_{0}^{*}$ & Oxygen vacancy \\
\hline $\mathrm{V}_{\mathrm{O}}^{*}$ & Oxygen vacancy with one electron \\
\hline $\mathrm{V}_{\mathrm{O}}$ & Oxygen vacancy with two electrons \\
\hline$V_{R}$ & Repulsive force \\
\hline $\mathrm{V}_{\mathrm{S}}$ & Sedimentation velocity \\
\hline $\mathrm{V}_{\mathrm{T}}$ & Total potential energy \\
\hline $\mathrm{V}_{\mathrm{Zn}}$ & Zinc vacancy \\
\hline VCM & Volume computational model \\
\hline XPS & X-ray photoelectron spectroscopy \\
\hline XRD & X-ray diffraction \\
\hline$\zeta$-potential & Zeta potential \\
\hline Zip & Zrt-like and Irt-like protein family \\
\hline $\mathrm{Zn}^{2+}$ & ionic zinc \\
\hline $\mathrm{Zn}_{\mathrm{i}}$ & Zinc interstitial atom \\
\hline $\mathrm{ZnO}$ & Zinc Oxide \\
\hline
\end{tabular}


$\mathrm{Zn}-\mathrm{OH}$

$\mathrm{Zn}-\mathrm{O}^{-}$

$\mathrm{Zn}-\mathrm{O}-\mathrm{Zn}$

$\mathrm{Zn}-\mathrm{OH}^{2+}$

$\mathrm{Zn}-\mathrm{OR}$

ZnT
Zinc hydroxide or neutral $\mathrm{ZnO}$ surface layer

Negatively charged $\mathrm{ZnO}$ surface layer

Zinc - oxygen - zinc nanoparticle nuclei

Positively charged $\mathrm{ZnO}$ surface layer

Zinc alkoxide

Zinc transporter family 


\section{CHAPTER 1 INTRODUCTION}

\section{Zinc Oxide Nanoparticles - Cancer Therapeutic or Environmental Health Crisis?}

Zinc oxide nanoparticles $(\mathrm{nZnO})$ are used worldwide in numerous applications and have a market value estimated at approximately $\$ 2.1$ billion. The $\mathrm{nZnO}$ market is forecasted to exceed $\$ 7.5$ billion by 2022 [1]. Figure 1.1 illustrates the consumer usage by region and highlights the global distribution of $\mathrm{nZnO}$. The industrial and research focus on $\mathrm{nZnO}$ encompasses nearly every consumer space and on any given day, the average person will likely interact with many of these products (Figure 1.2). Indeed, $\mathrm{nZnO}$ are ubiquitous in products which impact human health and lifestyle. Significant advancement in the research areas of biomedicine, electronic devices, and energy storage, along with the growing pharmaceutical and cosmetic industry currently drives the demand for the nearly $10^{5}$ tons of $\mathrm{nZnO}$ manufactured each year $[1,2]$.

Some nanomaterials show promise as potential drug delivery agents or cancer therapeutics due to their small sizes $(0-100 \mathrm{~nm})$ and their ability to be functionalized to target cell receptors and improve cellular uptake. In cancer research, $\mathrm{nZnO}$ has garnered much attention due to its selective toxicity towards a wide range of malignant cell types when compared to primary cells of the same lineage [3-6]. In contrast, other works demonstrate that $\mathrm{nZnO}$ is equally toxic to primary cells lines and multiple organisms [711]. Such disparate results leave the research world divided - is $\mathrm{nZnO}$ a promising cancer therapeutic or a toxic agent leading to a potential environmental health crisis? The ultimate 
answer to both questions is most likely yes. Without extensive studies to elucidate the complex mechanisms that govern its inherent toxicity, $\mathrm{nZnO}$ could become an environmental problem. However, with an understanding of how to tailor $\mathrm{nZnO}$ fabrication to control its toxicity, potential therapeutic uses are possible. Achieving this endpoint will be a challenging endeavor. Interdisciplinary, collaborative efforts will be required to gain a better understanding of mechanisms of toxicity and the long-term consequences of direct exposure of cells to $\mathrm{nZnO}[12]$.

\section{nZnO Material Synthesis}

Since the inception of nanotechnology research in the 1970's, considerable attention has been devoted to the development of novel NP fabrication methods [13] which can be grouped into three broad classifications (physical, liquid and gas) based primarily on the reaction phase of the method.(Table 1.1). Physical phase methods are typically top down approaches to NP fabrication. The one physical phase model in Table 1.1 is the mechanochemical method that breaks down bulk zinc oxide into smaller nanosized particles, and uses the addition of molecular and chemical compounds to influence the $\mathrm{nZnO}$ material properties [14-18]. In comparison, the remaining methods are bottom up approaches that form nanomaterials from the atomic level.

Synthesis methods in the liquid phase offer the advantage of relatively inexpensive

precursors and solvent systems without the need for expensive fabrication systems. The simplest of these is the precipitation method which involves a zinc precursor salt, such as $\mathrm{Zn}\left(\mathrm{NO}_{3}\right)_{2}$, dissolved in a basic solution (e.g. $\left.\mathrm{NaOH}\right)$ [19-21]. These reactions normally proceed at low temperatures over extended periods of time. Surfactants or other organics 
may be used to limit NP growth or modify the NP surface during the ripening stage of the $\mathrm{nZnO}$ fabrication [19-21].

Another widely used method, sol-gel synthesis, involves the forced hydrolysis of zinc precursors such as metal halogens $(\mathrm{M}-\mathrm{Cl})$, alkoxides $(\mathrm{M}-\mathrm{OR})$ or weak acids $(\mathrm{M}-$ $\mathrm{OOCCH}_{3}$ ) to form a colloidal $\mathrm{ZnO}$ sol within the solvent system (Eq. 1.1). This is followed by the condensation (Eq. $1.2 \& 1.3)$ of zinc hydroxide $(\mathrm{Zn}-\mathrm{OH})$ intermediates to produce $\mathrm{Zn}-\mathrm{O}-\mathrm{Zn}$ nuclei [22-27]. During the NP growth phase, these $\mathrm{Zn}-\mathrm{O}-\mathrm{Zn}$ building blocks combine to form gels in a continuous liquid phase. The final fate of the synthesis depends on the desired downstream application of the NPs as these gels can be precipitated to form particles or used to create thin films or ceramics [2].

$$
\begin{array}{lr}
\mathrm{Zn}-\mathrm{OR}+\mathrm{H}_{2} \mathrm{O} \rightarrow \mathrm{Zn}-\mathrm{OH}+\mathrm{ROH} & \mathrm{Eq}, 1.1 \\
\mathrm{Zn}-\mathrm{OH}+\mathrm{OH}-\mathrm{Zn} \rightarrow \mathrm{Zn}-\mathrm{O}-\mathrm{Zn}+\mathrm{H}_{2} \mathrm{O} & \mathrm{Eq}, 1.2 \\
\mathrm{Zn}-\mathrm{OR}+\mathrm{OH}-\mathrm{Zn} \rightarrow \mathrm{Zn}-\mathrm{O}-\mathrm{Zn}+\mathrm{ROH} & \mathrm{Eq}, 1.3
\end{array}
$$

Common variations of this method include the addition of $\mathrm{LiOH}$ to an ethanolic zinc acetate solution [28], or zinc acetate combined with a polyols such as diethyleneglycol (DEG) $[29,30]$. In the first method, the basic addition of the $\mathrm{LiOH}$ facilitates the hydroxide formation followed by the condensation reaction. Afterwards, the resulting gel is aged in an alkane solvent such as $n$-heptane to precipitate the particles [28]. In the later synthesis, Caruntu et al. (2002) proposed that the zinc acetate precursor forms a complex with a DEG dianion prior to hydrolysis. The subsequent condensation reaction releases the dianion leaving the $Z n-O-Z n$ species. Nanoparticles made with this method then rapidly precipitate out of the gel in the polar environment of the DEG solvent [30]. 
Solvothermal techniques employ varying degrees of temperature (typically between $100{ }^{\circ} \mathrm{C}$ to $1000^{\circ} \mathrm{C}$ ) and pressure (normally between $1 \mathrm{~atm}$ and 10,000 atm) within reaction vessels to convert synthesis precursors to $\mathrm{nZnO}[2,31-34]$. When water is used as a solvent, the method is referred to as hydrothermal. Nanomaterials fabricated in this manner are favored for electronic and optical applications as they often possess thermodynamically stable and metastable states [2]. Finally, microemulsions are created by combining an aqueous layer, an oil layer and a surfactant to create stable, transparent isotropic liquids [2, 35-37]. When the synthesis precursor and solvent are added to this liquid, microemulsions form spontaneously with NP formation occurring when synthesis precursor and solvent exchange takes place within the droplets [35].

The appeal of liquid phase methods is the ability to control the size and morphology of the $\mathrm{nZnO}$ through simple modifications in the synthesis method such as water addition, temperature and pressure control, capping agents or particle precipitation in organic solvents [22, 28-30, 38-42]. Additionally, these fabrication methods can result in defect states needed for effective photocatalysis $[22,43,44]$. The primary disadvantage is the low production yield of nanomaterial especially when compared to large scale industrial processes [22].

Gas phase synthesis methods (i.e. inert gas condensation and flame spray pyrolysis) both result in the zinc precursor entering a gaseous phase prior to NP formation and collection [45]. During inert gas condensation, the synthesis precursor is vaporized in the presence of an inert gas using a high frequency induction coil [46]. Upon leaving the induction system, the gaseous precursor is carried by convection in the inert gas to a cooling tank where particle formation and collection occurs [46]. An alternative gas phase method 
is high temperature flame spray pyrolysis. In this technique (Figure 1.3), the synthesis precursor immediately evaporates once it is injected into the high temperature flame. Following atomization, individual molecules participate in molecular collisions or nucleation driven events to initiate particle formation $[47,48]$. In the lower temperature

environment, further from the flame, formed particles undergo agglomeration followed by collection on a filter [49-51]. FSP is favored in industrial settings because of its simplistic methodology with fewer process steps, scalability, easy collection systems, high yield, and purity of the final product [47-52]. Varying the burner dimensions, precursor concentration, injection rate of the precursor, and composition of the carrier gas change the reaction dynamics in the FSP method and provide synthesis control to obtain the desired product $[48,50-52]$. Additionally, silica vapor may be introduced during the coagulation phase to achieve silica $\left(\mathrm{SiO}_{2}\right)$ coated nanomaterials $[53,54]$.

\section{Electronic Structure and Surface Reactivity}

$\underline{\text { Intrinsic Surface Defects and Photocatalytic Activity }}$

In the electronic band structure of semiconductors, the highest occupied energy band is the valence band (VB) while the lowest occupied level is the conduction band (CB) with the difference between the VB top and the CB bottom defined as the electronic band gap $\left(E_{g}\right)[55]$. When $\mathrm{nZnO}$ is excited with an energy source greater than or equal to the $\mathrm{E}_{\mathrm{g}}$, four potential photophysical processes may occur (Figure 1.4). Process I occurs when an electron is excited from the VB to an energy level within the CB (Figure 1.4, gray arrows). This excitation results in valence band holes $\left(\mathrm{h}_{\mathrm{vb}}^{+}\right)$and conduction bands electrons $\left(\mathrm{e}_{\mathrm{cb}}^{-}\right)$. The $\mathrm{e}_{\mathrm{cb}}^{-}$are very unstable, however, and undergo relaxation and recombination with the $\mathrm{h}_{\mathrm{vb}}^{+}$. Additionally, $\mathrm{e}_{\mathrm{cb}}^{-}$can undergo non-radiative energy release (blue arrows) from higher 
CB levels to the CB bottom followed by a radiative energy transfer to the top of the VB. This dissipation of light energy, termed the band -band photoluminescence process or process II (Figure 1.4, green arrow), produces the characteristic photoluminescence emission of $\mathrm{nZnO}$ at the $\mathrm{E}_{\mathrm{g}}$ of $\sim 3.3 \mathrm{eV}$ [55]. In the excitonic photoluminescence process III (red arrow), a non-radiative transition from the CB bottom to a sub band, proceeds the radiative emission to VB top. It is generally believed that intrinsic defects can trap $\mathrm{e}_{\mathrm{cb}}^{-}$and contribute strongly to excitonic emissions [55]. Finally, process IV (blue arrows) refers to non-radiative energy transitions from the $\mathrm{CB}$ to the VB.

Chemical catalysis utilizes the presence of a catalyst to intensify a catalytic reaction. This process depends on the ability of the catalyst to absorb the supplied energy and facilitate the desired reaction. By measuring how fast the catalysis proceeds, a kinetic constant can be determined to rate the efficiency of the catalyst. The band gap of $\mathrm{nZnO}$ $(\sim 3.3 \mathrm{eV})$ can be exceeded with UV radiation or ultrasonic energy making it an ideal catalyst. Photoexcitation employs the use of either UV-A or near ultraviolet $(315-400$ $\mathrm{nm})$ or UV-B or medium ultra violet $(280-315 \mathrm{~nm})$ irradiation $[56,57]$. Sonoexcition involves the application of ultrasonic energy $(20 \mathrm{kHz}-1 \mathrm{GHz})$ to an aqueous suspension of the $\mathrm{nZnO}$. This facilitates the formation and growth of acoustic cavitation microbubbles [58]. The inertia of the surrounding medium exerts high pressure and temperature on the microbubbles causing them to implode and emit picosecond bursts of sonoluminescent light [59]. Successive microbubble expansion and collapse (emission) result in sufficient energy to excite the semiconductor bandgap [59] (Figure 1.5).

The valence band holes $\left(\mathrm{h}_{\mathrm{vb}}^{+}\right)$and conduction band electrons $\left(\mathrm{e}_{\mathrm{cb}}^{-}\right)$produced upon excitation freely participate in redox reactions at the NP surface (Figure 1.5) [43, 60]. As 
good reducers, $\mathrm{e}_{\mathrm{cb}}^{-}$react with adsorbed $\mathrm{O}_{2}$ to produce the anionic superoxide radical $\left(\cdot \mathrm{O}_{2}^{-}\right)$, whereas $\mathrm{h}_{\mathrm{vb}}^{+}$, good oxidizers, will react with surface adsorbed hydroxide ions or water to produce hydroxyl radicals $(\cdot \mathrm{OH})[43,60,61]$. The superoxide radical $\left(\cdot \mathrm{O}_{2}^{-}\right)$further reacts to form hydrogen peroxide $\left(\mathrm{H}_{2} \mathrm{O}_{2}\right) . \mathrm{H}_{2} \mathrm{O}_{2}$ can then be further reduced to produce additional stores of $\mathrm{OH}^{-}$and $\cdot \mathrm{OH}$. Additionally, the superoxide $\left(\cdot \mathrm{O}_{2}^{-}\right)$can undergo oxidation to produce the perhydroxyl radical $(\cdot \mathrm{OOH})$ which reacts with $\mathrm{H}^{+}$and $\mathrm{e}_{\mathrm{cb}}^{-}$to produce more $\mathrm{H}_{2} \mathrm{O}_{2}$ [62].

Nanomaterials with intrinsic defects often exhibit faster catalytic rates $[43,44,55$, 60]. Metastable defects such as oxygen vacancies or interstitial oxygen are highly efficient at trapping photoelectrons observed in process III photoluminescence [55, 63]. Oxygen vacancies $\left(\mathrm{V}_{\mathrm{O}}^{\bullet}\right)$ can trap one or two electrons (Eq. 1.4), whereas interstitial oxygen anions $\left(\mathrm{O}_{\mathrm{i}}^{\prime \prime}\right)$ combine with $\mathrm{h}_{\mathrm{vb}}^{+}($Eq. 1.5$)$

$$
\begin{aligned}
& V_{o}^{*}+e_{c b}^{-} \rightarrow V_{o}^{\cdot}+e_{c b}^{-} \rightarrow V_{O} \\
& O_{i}^{\prime \prime}+h_{v b}^{+} \rightarrow O_{i}^{\prime}
\end{aligned}
$$

The electrostatic forces between the surface state and photoelectrons reduce electron - hole recombination rates extending the lifetime of these excitons and improving their catalytic ability $[43,55,64]$. Moreover, acceptor $\left(\mathrm{O}_{2}\right)$ and donor $\left(\mathrm{OH}^{-}\right)$molecules will chemically adsorbed to the defect states resulting in superoxide $\left(\cdot \mathrm{O}_{2}^{-}\right)$and hydroxyl $(\cdot \mathrm{OH})$ radicals $($ Eq. $1.6 \& 1.7)[44,63]$

$$
\begin{aligned}
& V_{O}^{\cdot}+O_{2} \rightarrow V_{O}^{\ddot{O}}+\cdot O_{2}^{-} \\
& O_{i}^{\prime}+O H^{-} \rightarrow O_{i}^{\prime \prime}+\cdot O H
\end{aligned}
$$

Modifications to $\mathrm{nZnO}$ synthesis methods designed to improve their catalytic activity and antimicrobial properties has been an active area of interest [39-42, 65-70]. 
Changing the overall synthesis method [40, 41, 69], solvent system [39, 42], precursor concentration [68] and synthesis temperature [68] have all been shown to significantly change the photo reactivity of $\mathrm{nZnO}$ and its microbial toxicity. Additionally, nanomaterials with multiple defect states have been shown to participate in abiotic ROS producing reactions at the NP surface without UV irradiation [43, 60, 61]. Even with antimicrobial studies demonstrating strong connections between NP-induced toxicity and photo reactivity, photocatalytic assays and defect state assessments are rarely performed in mammalian systems.

\section{Zeta Potential}

When fabricated on the nanoscale, $\mathrm{ZnO}$ develops high surface area to volume ratios, and NP surfaces become modified with charged ions or functional groups left behind from the synthesis. The addition of these groups and negatively charged surface defects modifies the overall charge density of the NPs. Once dispersed in an aqueous solution, adsorption of solvent ions and NP surface ionization creates an enhanced surface charge resulting in an electrical potential difference between the aqueous solution and the slipping plane surrounding the nanoparticle [43]. Often referred to as surface charge or zeta potential ( $\zeta$-potential), these measurements assess the electrophoretic mobility of nZnO within the dispersive media. Another useful measure is the isoelectric potential (IEP) or the $\mathrm{pH}$ at which the NPs will have a $\zeta$-potential of zero. At the IEP, nZnO has neutral OH groups resulting in $\mathrm{Zn}-\mathrm{OH}$ bonds. As the $\mathrm{pH}$ of the dispersive media increases above the IEP, adsorbed $\mathrm{H}^{+}$ions will move into the media thus forming negatively charged $\mathrm{Zn}-\mathrm{O}^{-}$ surface bonds [43]. Below the IEP, $\mathrm{H}^{+}$ions will transfer to the NP surface resulting in 
$\mathrm{Zn}-\mathrm{OH}^{2+}$ bonds and a positive surface charge. Since $\mathrm{nZnO}$ typically has an IEP at a $\mathrm{pH}$ between 8 and 10, the resulting $\zeta$-potential will be positive at physiologic $\mathrm{pH}$.

Studies connecting NP-induced toxicity to electrophoretic mobility remain inconclusive. Cationic NPs have demonstrated the potential to interact strongly with anionic cell membranes causing increased hole formation [71], endocytosis [72, 73] , phagocytosis [74], membrane depolarization [75], and passive entry into the cells $[71,76]$. Bhattacharjee et al. (2010) demonstrated this contention with differentially coated silica NPs resulting in positively $\left(\mathrm{Si}-\mathrm{NH}_{2}\right)$, neutrally $\left(\mathrm{Si}-\mathrm{N}_{3}\right)$ and negatively $(\mathrm{Si}-\mathrm{COOH})$ charged surfaces. The cationic silica NPs demonstrated more toxicity, intracellular ROS production, and phagocytosis in rat alveolar NR8383 macrophages with $\mathrm{Si}-\mathrm{NH}_{2}>\mathrm{Si}-\mathrm{N}_{3}>$ Si-COOH for all measured variables [74]. In a similar study using a non-phagocytic cell line (SK-BR-3 breast cancer cells), positive charged gold NPs showed significantly increased amounts of membrane-association and endocytotic uptake when compared to neutral or negatively charged NPs [77].

In contrast, Wingett et al. (2016) demonstrated that cellular toxicity increased with decreasing electrophoretic mobility $(-9 \mathrm{mV} \approx-30 \mathrm{mV}>+44 \mathrm{mV})$ [78]. Furthermore, modeling studies comparing the uptake of cationic and anionic NPs with neutral bilayers showed faster and more complete disruption of the bilayer with negatively charged NPs [72]. One limitation of many of these surface charge studies is the absence of experiments accessing NP dispersion and agglomeration behavior. Indeed, Wingett et al. (2016), demonstrated that their negatively charged NPs formed smaller agglomerates and decreased sedimentation when compared to the cationic NPs. Therefore, it is highly probable that this improved dispersion stability would increase cellular interactions of the 
anionic NPs thereby increasing their toxicity by a mechanism that is unrelated to surface charge [78].

\section{Agglomeration Behavior}

\section{Colloidal Stability}

The DLVO theory, named for Derjaguin, Landau, Verwey, and Overbeek, provides a mathematical model to describe the stability of colloidal suspensions (Figure 1.6). Dispersion stability depends upon the balance of two competing forces, electrostatic repulsion (blue dotted line) and van der Waals attraction (red dashed line). As two NPs draw close to one another, their ionic double layers will begin to overlap creating a repulsive force $\left(\mathrm{V}_{\mathrm{R}}\right)$. Meanwhile, attractive Van der Waal forces $\left(\mathrm{V}_{\mathrm{A}}\right)$ occur between the induced dipoles on the particles. The total potential energy of the dispersion $\left(\mathrm{V}_{\mathrm{T}}\right.$; solid purple line) represents the summation of these opposing forces. As seen in Figure 1.6, when the attractive force is greater than the repulsive force, a deep attractive well, termed the primary minimum, results. At larger distances, the repulsive force is larger than the attractive force creating a maximum positive energy barrier followed by a shallow secondary minimum [79].

For NPs to agglomerate and sediment out of the colloid, the NPs must have sufficient kinetic energy to overcome the energy barrier. Colloidal stability can be lost through various mechanisms. First, if the NPs have enough kinetic energy to overcome the energy barrier, the NPs will irreversibly agglomerate due to net attractive forces, become trapped in the deep potential well and undergo precipitation [79]. If the energy barrier is sufficiently large due to strong repulsive forces, the particles rebound yet may remain in the secondary minimum. Here particles are held together by much weaker bonds and tend 
to flocculate or form loosely packed NP agglomerates. Flocculation is reversible and can be overcome if enough dispersive energy is supplied. However, if flocculation becomes extensive the NPs could gain enough kinetic energy to overcome the barrier, due to their diffusion velocity and increasing mass, resulting in irreversible agglomeration. [79].

Finding ways to improve repulsive forces between NPs while minimizing attractive forces can be challenging. One way to accomplish this task is to introduce a form of steric repulsion to the NP system by means of a surface coating (Figure 1.6). Surface coating improves colloidal stability by shielding reactive NP surfaces thus prohibiting Van der Waals forces or by increasing repulsive forces through surface charge modulation.

Cho et al. (2011) highlighted the importance of dispersion stability by examining the relationship between diffusion and sedimentation velocity in NP colloids using an adherent cell model. In solution, NPs will move either by diffusion through the media in any direction or by sedimentation to the bottom of the cell well. Diffusion velocity $\left(V_{D}\right)$ is determined primary by the diffusion coefficient (D) which is inversely proportional to the hydrodynamic diameter $\left(\mathrm{d}_{(\mathrm{h})}\right)($ Eq. 1.8). Therefore, as the diameter increases the diffusion velocity decreases. The sedimentation velocity $\left(\mathrm{V}_{\mathrm{S}}\right)$ is also strongly influenced by the hydrodynamic diameter, but since this term is squared $\left(\mathrm{d}_{(\mathrm{h})}^{2}\right)$, even small increases in hydrodynamic diameter will exponentially increase the rate of NP sedimentation (Eq. 1.9). By looking at the ratio of sedimentation to diffusion (Eq. 1.10), Cho et al. developed a quantitative measure of NP stability with smaller ratios representing more stable suspensions [80].

$$
\begin{array}{lr}
V_{D}=\frac{2 D}{x}, \text { where } D=\frac{k_{B} T}{3 \pi \eta\left(d_{(h)}\right)} \text { and } \mathrm{x}=\text { distance traveled by the NPs } & \text { Eq, } 1.8 \\
V_{S}=\frac{2 g\left(\rho_{Z n o, a}-\rho_{m}\right) d_{(h)}^{2}}{9 \eta} & \mathrm{Eq}, 1.9
\end{array}
$$


$\frac{V_{S}}{V_{D}}$

Eq, 1.10

In their model, they evaluated coated and uncoated gold NP (AuNP) uptake in adherent cells grown in a traditional adherent model and cells grown on a glass slide that had been inverted within the cell well (Figure 1.7). They determined that NP suspensions with strong diffusion tendencies and low sedimentation (small $V_{S} / V_{D}$ ratios) showed no appreciable differences in NP uptake between the two different cell culture models; however, for unstable dispersions, higher $\mathrm{V}_{\mathrm{S}} / \mathrm{V}_{\mathrm{D}}$ ratios, the traditional model demonstrated significantly more NP uptake compared to the inverted model. Furthermore, polyethylene glycol (PEG) coating reduced the sedimentation velocity of the suspensions due to reduced attractive forces and increased steric repulsion [80].

They hypothesized that the differences observed favoring the traditional adherent model were a result of excessive amounts of NPs in the unstable suspensions driven by sedimentation onto the cell layer resulting in significant increases in uptake (Figure 1.8) [80]. This same reasoning can be applied to a suspension cell model. Nanoparticles that are dominated by strong diffusion tendencies will be free to interact with the suspended cells. Unstable dispersions dominated by sedimentation will result in less NP bioavailability. Both potential outcomes, excessive NP sedimentation onto the adherent cell layer or less NP bioavailability in suspension cells, will result in inconsistent results.

\section{$\underline{\text { Strategies to Achieve Dispersion Stability }}$}

During in vitro studies, the treatment condition must remain bioavailable to the treated cells over an extended period. For many drug or chemical treatments, this requires finding the appropriate solution to solubilize the therapeutic agent. Since NPs remain in particle form in suspension, the goal then becomes creating NP dispersions without 
appreciable agglomeration or sedimentation. Achieving this requires management of the complex interplay between NP agglomerate and complex formation (hydrodynamic diameter) and their sedimentation behavior over time [81]. Factors that influence dispersion stability are numerous and include NP size [82, 83], surface structure [84, 85], capping agents [86], solution $\mathrm{pH}$ and ionic strength [79], organic and protein components [83, 85, 87], and free ions in the solution system [88]. To this end, numerous investigations have employed various coating materials, such as polyethylene glycol (PEG) [80], trinoctylphosphine oxide (TOPO) [86], silica $\left(\mathrm{SiO}_{2}\right)$, and poly (acrylic acid) (PAA) [78], to increase steric stabilization of the NP surface.

The drawback of this approach is that it changes the NP surface reactivity and adds a layer of complexity to NP-cell interactions. Therefore, it is necessary to find a means to stabilize particles that does not involve a chemical or physical change to the pristine NP surface and is compatible with cells. Additionally, any method developed should ideally be transferable to an in vivo model [85]. In most in vitro experiments, cells are treated in a culture medium to which NPs are introduced in a prepared dispersion. Once NPs are introduced, a biomolecular corona consisting of proteins and ionic complexes from the cellular media will quickly form [79, 88-92]. Biomolecular coronas appear to be multidimensional consisting of a tightly bound near monolayer called a hard corona underneath a dynamic and rapidly changing soft corona [90, 93]. Typically, protein components make up the bulk of the hard corona providing steric stabilization [94], while other molecular species comprise the soft corona and influence NP-cell interactions [83, $88,89]$. 
Given this, it is probable that cellular media proteins such as fetal bovine serum (FBS), may provide a solution to dispersion instability with uncoated NPs. Indeed, studies have shown that NPs introduced to media without FBS develop highly ionic coronas and unstable colloids. However; suspending NPs in FBS prior to dispersion in the desired solution would provide steric stabilization and reduce agglomeration. Utilizing this approach could allow the use of ionic solutions such as PBS for NP stock solutions without unwanted dispersion instability.

\section{Dissolution Potential}

Once dispersed, nZnO will undergo dissolution and leach zinc ions into the dispersion media. Several solution-related factors including the ionic strength, $\mathrm{pH}$, concentration, temperature and solvated constituent molecules influence the degree to which dissolution occurs $[95,96]$. The presence and composition of these solvated molecules is a strong determinant of a solution's ability to solubilize $\mathrm{nZnO}[97,98]$. $\mathrm{ZnO}$ NPs in media with low levels of phosphate (DMEM) appear to dissolve to a higher extent than those with high phosphate levels (RPMI). However, ionic zinc rapidly reacts with phosphate to form highly insoluble zinc phosphate that rapidly precipitates from the dispersion [99, 100] making $\mathrm{nZnO}$ dissolution comparisons across multiple solutions difficult. More recent research suggests that non-essential media additives significantly increase NP dissolution, alter the biomolecular corona composition and structure, and increase NP-induced toxicity [99]. Additionally, amino acid rich solutions can also increase

dissolution whereas protein components, such as FBS or BSA, may decrease dissolution if sufficient NP coating results [95]. Given this complexity, the dissolution balance of NP 
dispersions could be one of reduced solubility through protein stabilization of NP clusters or increased dissolution though ligand-mediated processes [95].

The physicochemical properties of the $\mathrm{nZnO}$ can also alter their dissolution potential including size, shape, zeta potential and transition metal doping [10, 101-103]. Hydrodynamic size is perhaps the most important material property determinant of NP solubility. Indeed, David et al. (2012) demonstrated that NP dispersions eventually reach a steady state condition where the dissolution kinetic balance is entirely dependent upon the radius of the NP agglomerates and the concentration [104]. Furthermore, they assessed dissolution across multiple NP samples and concluded that any observed dissolution trends could be explained by their mathematical model [105]. Therefore, it remains imperative that NP studies characterize all material properties and media components that have the potential to influence agglomeration potential and thus NP dissolution.

Introducing cells to a rapidly changing NP dispersion presents multiple challenges in determining the active $\mathrm{ZnO}$ species encountered by the cells. Once dispersed into cellular media, $\mathrm{nZnO}$ will leach ionic zinc and change the dynamics of the colloidal dispersion. The original mass of zinc now exists in both ionic and non-ionic forms (Figure 1.9). Inevitably, some of this extracellular $\mathrm{Zn}^{2+}$ will enter cells to become intracellular free $\mathrm{Zn}^{2+}$ within the cytosol or endocytotic vesicles [106-108] (right side of Figure 1.9). Additionally, liberated $\mathrm{Zn}^{2+}$ reacts freely with carbonate and phosphate anions to produce partially insoluble molecular zinc carbonates and phosphates [109]. These insoluble species along with other media components, surface adsorb to large agglomerates of intact nZnO (i.e., nonionic zinc) to form amorphous complexes of encapsulated $\mathrm{ZnO}[107,110$, 111] (left side of Figure 1.9). Zinc molecular species conversion and $\mathrm{ZnO}$ complex 
formation is a dynamic process for up to 4 to 6-hours post-treatment and needs to be considered in comprehensive nanotoxicology studies.

\section{Inherent Toxicity of $\mathrm{nZnO}$}

The cytotoxicity of $\mathrm{nZnO}$ has been extensively studied in multiple mammalian cell types representing nearly every major organ system [3, 4, 8, 11, 67, 112-123]. Additionally, $\mathrm{nZnO}$ toxicity has been observed in microbial systems $[6,41,42,67,70,124]$, zebra fish $[7,125,126]$, yeast $[127,128]$, nematodes [129], microalgae [130, 131], and crustaceans [132]. To this extent, the inherent toxicity of $\mathrm{nZnO}$ is well established; however, the underlying mechanism of $\mathrm{nZnO}$ toxicity remains elusive. Strong evidence suggests that nZnO-induced apoptotic cell death is secondary to processes such as cellular ROS formation, antioxidant systems imbalance, and overwhelming oxidative stress $[3,6,43,93$, 133-136]. Efforts to assign specific material properties or even underlying cellular processes to the increased ROS production have been inconclusive. Surface property studies suggest that abiotic ROS production and dissolution at reactive NP surfaces lead to lipid peroxidation and cell membrane disruptions [6, 11, 104, 137]. Alternatively, large influxes of ionic zinc from both extracellular and intracellular pools are known contributors to mitochondrial dysfunction and intracellular ROS production [102, 135, 138-141]. These seemingly opposing views of $\mathrm{nZnO}$-induced cytotoxicity has resulted in a polarized research community.

\section{Surface Property Contributions to nZnO Toxicity and Oxidative Stress}

Several studies attribute $\mathrm{nZnO}$ toxicity to their capacity to generate either abiotic ROS at the NP surface or intracellular ROS resulting in oxidative stress leading to apoptosis and cell death. Several factors contribute to the surface reactivity and abiotic 
ROS generation of $\mathrm{nZnO}$. The narrow electronic band structure of $\mathrm{nZnO}$ allows for photoor ultrasonic excitation to create $\mathrm{h}_{\mathrm{vb}}^{+}$and $\mathrm{e}_{\mathrm{cb}}^{-}$redox pairs capable of radical formation. Additionally, discontinuous crystal planes facilitate the adsorption of ionic functional groups [61]. Finally, altered electronic states secondary to crystal defect states contribute to redox cycling at the NP surface (Figure 1.10A) [61]. These unique surface characteristics have been shown to increase the concentration of free radicals in NP dispersions capable of oxidizing lipids, proteins, DNA and other essential biomolecules [142].

ROS-induced lipid peroxidation results in the loss of cell membrane integrity facilitating the uptake of ROS species, NPs and ionized zinc (Figure 1.10B) [6, 115, 143]. Additionally, ROS may be taken up by cells through aquaporins or chloride channels (Figure 1.10C) [144]. Internalized ROS will cause oxidative damage to cellular components or activate stress-induced signaling, such as Nox, which produces ROS as a byproduct (Figure 1.10D) [115, 134, 145]. Genotoxicity, verified through DNA damage and cell cycle arrest, has been observed in multiple studies [117, 146, 147]. Normally, oxidative DNA damage is attributed to ROS; however, evidence also suggests that malondialdehyde (MDA), a lipid peroxidation byproduct, $[115,148]$ or ionic zinc also contribute to DNA damage $[117,146,147]$. Finally, changes in the NP charge density due to surface adsorbed groups and defect states can increase electrostatic interactions between normally positively charged $\mathrm{ZnO}$ NPs and the negatively charged cellular membrane leading to membrane damage or induced NP uptake [149-151].

Dissolution Contributions to $\mathrm{nZnO}$ Toxicity and Oxidative Stress

The solubility of $\mathrm{nZnO}$ in NP dispersions introduces multiple forms of $\mathrm{ZnO}$ and ionic zinc available for cellular interactions. Identifying toxicity mechanisms that consider 
the balance of all these species has created considerable debate in the research community. One proposed mechanism is that large pools of ionic zinc are liberated within the extracellular environment and then taken up by the cell primarily through non-specific ion transporters (Figure 1.11A) or specialized zinc importers termed Zips (Figure 1.11B) [106108]. Song et al. (2010) demonstrated this connection by analyzing $\mathrm{ZnCl}_{2}$ and commercially available $\mathrm{nZnO}$ samples. They found $\mathrm{ZnCl}_{2}$ to be significantly more toxic than all the NP samples. Furthermore, they discovered that the supernatants isolated after 24 hours from nZnO-treated cellular media were also toxic but to a lesser extent than the NP treated cells. As a result, they concluded that extracellular dissolution was primarily responsible for the NP-induced toxicity [106].

The alternative hypothesis suggests that NP-cell contact results in the active uptake of NP agglomerates in endocytotic vesicles (Figure 1.11C) followed by rapid dissolution of non-ionic zinc in the acidic endosome or through passive transport through the cellular membrane (Figure 1.11D) [23, 36, 39, 43]. Many of these studies are somewhat inconclusive as they have relied solely on endocytosis to explain the presence of intracellular zinc without offering direct evidence of the actual uptake mechanism or a comparative analysis of extracellular zinc studies. The strongest evidence for this model is observed in macrophage studies or other phagocytic cell types that can engulf large amounts of extracellular material through a variety of mechanisms [102, 141, 152, 153]. For example, Wang et al. (2014) used cellular uptake inhibitors to demonstrate that macrophages utilize both macropinocytosis and phagocytosis for NP uptake [141]. However, this mechanism would also result in the uptake of large nZnO agglomerates and extracellular $\mathrm{Zn}^{2+}$ making it difficult to attribute any intracellular zinc increases to one 
specific zinc source. Furthermore, these results may not be indicative of NP uptake mechanisms in non-phagocytic cell types. As an illustration, Othman et al. (2016), demonstrated that bare $\mathrm{nZnO}$ was selectively taken up by breast cancer cells through clathrin-independent endocytosis which resulted in gradual time-dependent increases in intracellular zinc, eventually leading to cell death [140]. However, they found a similar trend in cells treated with $\mathrm{ZnCl}_{2}$. Thus, they could not differentiate between intracellular ionic zinc resulting from $\mathrm{nZnO}$ uptake or $\mathrm{Zn}^{2+}$ entering the cell from the extracellular environment.

In non-phagocytic Jurkat cells, Buerki-Thurnherr et al. (2013) explored both dissolution models with bare and coated nZnO. They employed zinc chelators diethylenetriaminepentaacetic acid (DTPA) and $N, N, N^{\prime}, N^{\prime}$-tetrakis(2-pyridinylmethyl)1,2-ethanediamine (TPEN) for extracellular and intracellular zinc, respectively, while monitoring the corresponding ionic zinc levels and NP-induced toxicity. Both DTPA and TPEN chelation used independently completely abrogated NP-induced cytotoxicity. Similar results were obtained when cells were treated with equimolar concentrations of $\mathrm{ZnCl}_{2}$; however less toxicity was observed and significantly lower amounts of chelating agents were needed with the coated-nZnO. Ultimately, they concluded that NP-induced toxicity was primarily dependent upon extracellularly released zinc that induces cell death by apoptosis [12].

Once internalized, ionic zinc will cause a vast number of cellular responses. Initially, cells will attempt to regulate ionic zinc excesses through various mechanisms such as zinc transport out of the cell or into zincosomes via zinc-specific efflux proteins (ZnTs) (Figure 1.11E), and through metallothionein protein sequestration (Figure 1.11F) 
[154-159]. Inevitably, increasing ionic zinc levels will overwhelm the zinc homeostasis of the cells and cause toxic effects. Kao et al. (2011) established that increases in cytosolic zinc were systematically followed by elevated levels of mitochondrial zinc and collapse of the mitochondrial membrane potential (Figure 1.11G). Additional studies have also linked increased cytosolic zinc levels to mitochondrial swelling, mitochondrial membrane transition, impairment of inner membrane $\mathrm{H}^{+}$conductance, impaired respiration, adenosine triphosphate (ATP) depletion and cytochrome c release to signal apoptosis $[139,152,160$, 161]. Genotoxicity and organelle damage has also been linked to $\mathrm{nZnO}$ dissolution although it is unknown if this type of damage is a direct result of ionic zinc or related to substantial oxidative stress after mitochondrial dysfunction (Figure 1.11H).

\section{Cellular Regulation of and Response to Zinc and $\mathrm{nZnO}$}

\section{$\underline{\text { Maintaining Zinc Homeostasis }}$}

Zinc is an essential metal for humans with zinc deficiencies being linked to growth retardation, poor immune system function, delayed wound healing, loss of appetite, impaired taste acuity, reproduction issues, and impaired insulin function and blood glucose regulation $[162,163]$. As the only biologically essential transition metal that does not participate in redox activity, zinc is an ideal choice for use as a protein cofactor with approximately $10 \%$ or 2,800 human genes encoding for proteins with zinc -binding capability (Figure 1.12) [164]. It is estimated that the total amount of cellular regulated zinc is between $200-300 \mu \mathrm{M}$ [165], yet steady state concentrations of free ionic zinc are consistently measured in the nanomolar to picomolar range with variably dependent upon the zinc reserves needed for cellular function. To function as an effective second messenger, labile zinc stores have to remain at concentrations sufficient for use during 
signaling yet low enough to demonstrate the desired zinc level fluctuations without inhibition of other zinc-sensitive secondary messaging components [166]. For instance, skin $(32 \mu \mathrm{g} / \mathrm{g}$ zinc) and blood (5-6 $\mu \mathrm{g} / \mathrm{g}$ zinc) have strong immunological importance and low steady-state labile zinc; however, immune-privileged cells in the retina and prostate exhibit high useable zinc concentrations at 290 and $700 \mu \mathrm{g} / \mathrm{g}$ zinc, respectively [166]. Given the extensive involvement of zinc in cellular processes yet very low amounts of free zinc, tight cellular regulation is needed to ensure that sufficient amounts of zinc are available when needed.

Managing both steady state and non-steady state (i.e., deficiencies and excesses) levels of cytosolic zinc is accomplished by a considerable network of proteins responsible for zinc sensing, binding and transport in and out of the cytosol. Steady state control of zinc levels or intracellular zinc buffering is managed by a group of cytosolic zinc-binding proteins including the zinc sensor, metal-responsive transcription factor-1 (MTF-1), metallothioneins (MT), and various cell signaling mechanisms [154, 164]. Research suggests that MTF-1 has an affinity for ionic zinc in the low nanomolar range and controls the upper limit of steady state zinc concentrations. MTF-1 binds excess zinc in the cytosol, and translocates to the nucleus where it regulates the transcription of both metallothioneins and zinc tranporter-1 (ZnT1) through the metal responsive element (MRE) [156, 167-169]. Zinc deficiencies and excesses (non-steady state $\mathrm{Zn}^{2+}$ levels) are managed through a process termed muffling which results in the redistribution of intracellular zinc through the use of zinc transporters [164].

$\underline{\text { Metallothioneins and Zinc Transporters }}$ 
Metallothioneins (MTs) are small proteins whose cysteine-rich $\alpha$ and $\beta$ domains can effectively coordinate up to seven zinc ions [159]. Four isoforms and additional subisoforms exist in mammals, and differ in size, coordination ability, organism and tissue distribution [164]. In addition to zinc homeostasis, these proteins participate in metal detoxification and oxidative stress remediation. Heavy metal detoxification is mediated by MT's ability to effectively coordinate all group 11 and 12 metals with preferential binding towards more toxic metal species such as mercury, lead and arsenic. In the absence of heavy metals, MTs bind mostly $\mathrm{Zn}^{2+}$ or to a lesser extent $\mathrm{Cu}^{2+}$ or $\mathrm{Zn}^{2+} / \mathrm{Cu}^{2+}$ combinations [159]. When exposed to heavy metals, MTs release bound zinc triggering MTF-1 nuclear translocation and subsequent transcription of the MRE promoter. This results in increased transcription of the MT gene and upregulation of mRNA products.

The mechanism by which MTs mediate oxidative stress remains unclear. It has been hypothesized that bound ATP and glutathione (GSH) are required for MT coordination of $\mathrm{Zn}^{2+}$ and that ATP and GSH release are subsequently needed for zinc release from the proteins [170]. GSH neutralizes ROS to become oxidized glutathione disulfide (GSSG) and the GSH/GSSG is often used to measure the oxidative stress of cells. Activated MTF1 along with other transcription factors upregulate MT expression through the antioxidant response element (ARE) promoter located on the MT gene [156, 159, 171]. The abundant cysteine content in metal free (apo-) MT proteins leads to their enhanced reactivity with the oxygen-rich ROS. The ability of MTs to mediate hydroxyl radical damage has actually been shown to be functionally similar to superoxide dismutase in yeast cells [167]. Restoration of zinc homeostasis following oxidative stress remediation is eventually achieved through MT replenishment from increased mRNA translation or GSH reduction 
of the previously oxidized proteins [159]. While MTs assert many of their functions in the cytosolic space of cells, they can be imported into the mitochondrial intramembrane space and release zinc ions to inhibit mitochondrial respiration [164] (Figure 1.13).

Zinc importers (SLC39) and exporters (SLC30), commonly referred to as Zip and $\mathrm{ZnT}$ proteins, respectively, are a part of the solute-linked carrier family of proteins and participate in muffling processes to manage non-steady state concentrations of cytosolic zinc (Figure 1.13). Zips are responsible for zinc transport into the cytoplasm from extracellular spaces or from cellular organelles [172]. Fourteen importers (Zip1 - Zip14) have been identified in the human genome and consist of approximately eight transmembrane domains, a histidine-rich loop region and an amphipathic channel to allow ion transport [172]. Studies have demonstrated that zinc transport with Zip1 (SLC39A1), Zip2 (SLC39A2), and Zip8 (SLC39A8) is energy independent and likely stimulated with bicarbonate anions indicating a possible zinc/bicarbonate symport mechanism $[157,172]$. In addition to zinc responsiveness, most Zips also respond to other hormones and inflammatory cytokines [172]. ZnTs are responsible for zinc efflux to either the extracellular environment or endosomal compartments [157, 173]. The nine ZnTs (SLC30A1 - SLC 30A9) identified in humans have six or more transmembrane amphipathic domains that facilitate transport and are suspected to act as dimers and trimers [157]. ZnTs show strong sensitivity to dietary changes in zinc [157] and ZnT1 expression has been linked to changes in MT expression and MTF-1 [167].

Zinc Signaling and Influences on Zinc Homeostasis

In addition to buffering and muffling actions, several signaling pathways rely on zinc as a second messenger and alter intracellular zinc accordingly. Zn signaling falls into 
two broad categories termed early and late Zn signaling [174]. Early Zn signaling (Figure 1.14A) occurs in response to an external stimulus, such as ligand binding to a receptor, that results in the subsequent release of ionic zinc into the cytosol from common zinc stores such as metallothioneins or the endoplasmic reticulum. Early zinc signaling is transcription independent and normally occurs within minutes after the external stimulus [174]. Released zinc then acts as a second messenger to facilitate the desired cellular response. Yamasaki et al. (2008) illustrated how mast cells utilize ionic zinc release to facilitate cytokine production and secretion. They found that engagement of antigen-bound immunoglobulin $\mathrm{E}$ (IgE) with the high-affinity $\operatorname{IgE}$ receptor (FceRI) in mast cells resulted in large $\mathrm{Ca}^{2+}$ and mitogen-activated protein kinase kinase (MAPKK) dependent increases of intracellular zinc originating from the perinuclear region of the cells within several minutes of FCeRI stimulation [175]. Using the intracellular zinc chelators and calcium channel inhibitors, they demonstrated that this released zinc enhanced the $\mathrm{NF}-\kappa \beta$ binding of DNA needed for cytokine regulation $[175,176]$. In T cells, intracellular zinc increases via plasma membrane localized Zip 6 within one minute of T cell activation and influences CD69 and CD25 expression [177].

In contrast, late zinc signaling is transcription dependent and describes $\mathrm{Zn}$ facilitated cellular responses that occurs after the transcription of zinc transporters (Figure 1.14B). Toll-like receptor four (TLC4) is activated by the bacterial endotoxin lipopolysaccharide (LPS) in dendritic cells (DC) and initiates myeloid differentiation factor 88 (MyD88) and Toll/IL-1 receptor (TIR) domain-containing adapter inducing interferon $\beta$ (TRIF)-mediated signaling to induce DC maturation [178]. MyD88- and TRIP-mediated signaling results in the transcription of major histocompatibility complex (MHC)-II, 
upregulation of ZnT1, ZnT4 and ZnT6 and the suppressed transcription of Zip6 and Zip10 [178]. The net result of the Zip downregulation and ZnT upregulation would be an increase in zinc efflux from the cell to reduce cytosolic zinc concentrations. Indeed, experiments with an ionic zinc surplus or Zip6 overexpression decreased MHC-II trafficking to the plasma membrane and DC maturation [178]. These results linking LPS-induced expression of zinc transport proteins to intracellular zinc decreases and DC activation highlight late zinc signaling in these processes.

\section{$\underline{\text { ZnO Nanoparticles and Genetic Expression }}$}

Given the importance of zinc in cellular function, many researchers have sought to determine the genomic or proteomic consequences of excess ionic zinc or $\mathrm{nZnO}$ in mammalian cells [156, 179-184]. The observed genetic responses across these studies is widely variable, however, a few common themes exist. First, the differential expression of MTF-1, metallothioneins, and zinc responsive transport proteins was observed across numerous cell types, zinc concentration levels and time points underscoring the importance of these proteins in the regulation of steady-state zinc levels $[158,169,173,179-181,183$, 185]. In addition, differentially expressed genes associated with stress response pathways outweigh contributions from other cellular processes. In immune cells, apoptotic and cell death pathway genes are consistently upregulated or downregulated depending on the cell type or time of treatment $[181,183]$, and these results confirmed through proteomic studies [182]. The significant upregulation of mRNA and translation of chaperones and other proteins involved in proteasome degradation indicate that $\mathrm{nZnO}$ can disrupt normal protein folding or function [181-183]. Cell cycle [183] and metabolic impairment are evident through the upregulation of G1 phase mediator cyclin-dependent kinase inhibitor 1 
$(C D K N 1 A)$ and increased enzymatic activity in the glycolysis pathways post $\mathrm{nZnO}$ treatment [182]. Furthermore, high levels of the metabolite pyruvate following $\mathrm{nZnO}$ treatment is believed to function as a survival factor against zinc-induced stress [182]. Finally, ionic zinc is capable of mimicking other signaling molecules including cytokines, growth factors, hormones and influencing various intracellular signaling pathways [174, 186]. This could allow ionic zinc to directly bind to and alter the structure and biological function of several cytokines and growth factors [187].

\section{Moving Forward}

Controlling NP-induced toxicity is imperative for achieving safe and effective use of $\mathrm{nZnO}$ for either consumer or biomedical applications. To achieve this, a thorough understanding of the material properties that influence toxicity and a connection to observed cellular responses is required (Figure 1.15). Research has demonstrated that NPinduced toxicity can be altered through material property modifications such as transition metal doping or surface coatings to a single $\mathrm{nZnO}$ sample. This formulation controlled approach has been instrumental in identifying how certain physicochemical modifications result in cellular damage. These types of modifications, however, can permanently alter the intrinsic $\mathrm{nZnO}$ core or surface structure and often fail to explain how comparable studies using similar NP-modifications but differently synthesized $\mathrm{nZnO}$ samples often result in different cytotoxic responses.

To address these disparities, in 2012 the research community examined the importance of materials characterization and developed a minimal set of methods needed for every nanomaterial study. These characterizations included particle size and distribution, morphology, chemical composition and impurities, agglomeration tendencies, 
surface chemistry, surface area, surface reactivity and persistence [188]. Schrurs and Lison (2012) examined 38 research studies on silica nanoparticles across six different parameters and discovered significant disparity in the reported results in relation to cytotoxicity, cellular uptake, agglomeration potential, surface reactivity and redox potential. Two of these properties, agglomeration potential and surface reactivity, were found to be woefully underrepresented in the literature. For agglomeration potential, 19 studies documented stability in cellular media, four studies examined agglomeration tendencies, two studies evaluated the impact of stability on cytotoxicity, and only 13 measured surface reactivity [189]. Additionally, they found that very few authors arrived at the same conclusions as to which material properties most influenced NP-induced toxicity. Similar recommendations have been made for downstream biological experiments and include cell death, oxidative stress, proliferation, DNA damage, cell adhesion, inflammatory potential, NP uptake, energy metabolism and genetic expression assays $[190,191]$. However, more diversity is expected regarding these experiments as the anticipated cellular responses will vary with the biological model studied.

These discrepancies have resulted in uncertainty as to which specific material properties within a very diverse set of $\mathrm{nZnO}$ formulations have the greatest influence on toxicity. As an illustration, two comparable studies, George et al. (2010) and Thurber et al. (2012), examined the effect of Fe-doping in $\mathrm{nZnO}$ on the NP-induced cytotoxicity in different mammalian cell types. Both studies employed small, spherical nZnO $(<20 \mathrm{~nm})$ and similar Fe-doping percentages $(<10 \%)$. However, one study found that Fe-doping decreased both the NP-induced cytotoxicity and oxidative stress responses when compared to the un-doped $\mathrm{nZnO}[10]$, whereas the other study found increasing cytotoxic trends with 
the Fe-doping [5]. Interestingly, one major difference between the two studies was the nZnO synthesis method. Flame spray pyrolysis (FSP) was utilized in the first study [10], while a wet chemical method was employed for the later work [5]. This suggests that physicochemical differences between the two formulations may have been present before doping that could have potentially affected the outcome. Additionally, the characterization methods and cellular response assays differed between the two works which resulted in different conclusions as to the cause of the toxicity. Given these disparate results, it is difficult to make definitive conclusions regarding the effects of Fe-doping on NP-induced toxicity without first understanding how alterations in the synthesis method changed the underlying material properties of the $\mathrm{nZnO}$.

The inconsistencies observed in the literature indicate that a comprehensive investigation of the material properties that influence the toxicity of $\mathrm{nZnO}$ NP towards mammalian cells is needed. The overall objectives of the work presented here were to $i$ ) evaluate the physicochemical properties of differentially synthesized $\mathrm{nZnO}$ fabricated through both bottom-up and top-down methods and matched for size distribution, crystal structure and band gap, ii) reduce NP agglomeration tendencies within complex media and achieve dispersion stability, iii) define which material property interactions have the greatest potential to affect cellular toxicity, and to $i v$ ) examine the preferential toxicity of nZnO towards human leukemic cells through genetic expression studies. With this knowledge, it may be possible to systematically engineer and control the physicochemical properties of $\mathrm{nZnO}$ to maximize cell selectivity and cytotoxicity to achieve levels that are ideal for practical applications towards future biomedical use and environmental safety. 


\section{References}

[1] Sahu, Y. S. Nano zinc oxide market by application (paints \& coatings, cosmetics, and others) - global opportunity analysis and industry forecast, 2014-2022 2016, p. 99. (accessed 08/25/2017).

[2] Kołodziejczak-Radzimska, A.; Jesionowski, T. Zinc oxide-from synthesis to application: A review. Materials 2014, 7, 2833-2881.

[3] Hanley, C.; Layne, J.; Punnoose, A.; Reddy, K. M.; Coombs, I.; Coombs, A.; Feris, K.; Wingett, D. Preferential killing of cancer cells and activated human t cells using zno nanoparticles. Nanotechnology 2008, 19, 1-10.

[4] Hanley, C.; Thurber, A.; Hanna, C.; Punnoose, A.; Zhang, J. H.; Wingett, D. G. The influences of cell type and zno nanoparticle size on immune cell cytotoxicity and cytokine induction. Nanoscale Research Letters 2009, 4, 1409-1420.

[5] Thurber, A.; Wingett, D. G.; Rasmussen, J. W.; Layne, J.; Johnson, L.; Tenne, D. A.; Zhang, J. H.; Hanna, C. B.; Punnoose, A. Improving the selective cancer killing ability of zno nanoparticles using fe doping. Nanotoxicology 2012, 6, 440-452.

[6] Premanathan, M.; Karthikeyan, K.; Jeyasubramanian, K.; Manivannan, G. Selective toxicity of zno nanoparticles toward gram-positive bacteria and cancer cells by apoptosis through lipid peroxidation. Nanomedicine 2011, 7, 184-192.

[7] Bai, W.; Zhang, Z.; Tian, W.; He, X.; Ma, Y.; Zhao, Y.; Chai, Z. Toxicity of zinc oxide nanoparticles to zebrafish embryo: A physicochemical study of toxicity mechanism. Journal of Nanoparticle Research 2009, 12, 1645-1654.

[8] Brunner, T. J.; Wick, P.; Manser, P.; Spohn, P.; Grass, R. N.; Limbach, L. K.; Bruinink, A.; Stark, W. J. In vitro cytotoxicity of oxide nanoparticles: Comparison to asbestos, silica, and the effect of particle solubility. Environmental Science \& Technology 2006, 40, 4374-4381.

[9] Croteau, M. N.; Dybowska, A. D.; Luoma, S. N.; Valsami-Jones, E. A novel approach reveals that zinc oxide nanoparticles are bioavailable and toxic after dietary exposures. Nanotoxicology 2011, 5, 79-90.

[10] George, S.; Pokhrel, S.; Xia, T.; Gilbert, B.; Ji, Z. X.; Schowalter, M.; Rosenauer, A.; Damoiseaux, R.; Bradley, K. A.; Madler, L. et al. Use of a rapid cytotoxicity screening approach to engineer a safer zinc oxide nanoparticle through iron doping. ACS Nano 2010, 4, 15-29. 
[11] Sun, J.; Wang, S. C.; Zhao, D.; Hun, F. H.; Weng, L.; Liu, H. Cytotoxicity, permeability, and inflammation of metal oxide nanoparticles in human cardiac microvascular endothelial cells cytotoxicity, permeability, and inflammation of metal oxide nanoparticles. Cell Biology and Toxicology 2011, 27, 333-342.

[12] Buerki-Thurnherr, T.; Xiao, L.; Diener, L.; Arslan, O.; Hirsch, C.; Maeder-Althaus, X.; Grieder, K.; Wampfler, B.; Mathur, S.; Wick, P. et al. In vitro mechanistic study towards a better understanding of zno nanoparticle toxicity. Nanotoxicology 2013, 7, 402-416.

[13] Contado, C. Nanomaterials in consumer products: A challenging analytical problem. Frontiers in Chemistry 2015, 3, 1-20.

[14] Ralphs, K.; Hardacre, C.; James, S. L. Application of heterogeneous catalysts prepared by mechanochemical synthesis. Chemical Society Reviews 2013, 42, 7701-7718.

[15] Stanković, A.; Veselinović, L.; Škapin, S. D.; Marković, S.; Uskoković, D. Controlled mechanochemically assisted synthesis of zno nanopowders in the presence of oxalic acid. Journal of Materials Science 2011, 46, 3716-3724.

[16] Dodd, A.; McKinley, A.; Saunders, M.; Tsuzuki, T. Mechanochemical synthesis of nanocrystalline sno2-zno photocatalysts. Nanotechnology 2006, 17, 692-698.

[17] Ao, W.; Li, J.; Yang, H.; Zeng, X.; Ma, X. Mechanochemical synthesis of zinc oxide nanocrystalline. Powder Technology 2006, 168, 148-151.

[18] Moballegh, A.; Shahverdi, H. R.; Aghababazadeh, R.; Mirhabibi, A. R. Zno nanoparticles obtained by mechanochemical technique and the optical properties. Surface Science 2007, 601, 2850-2854.

[19] Khoshhesab, Z. M.; Sarfaraz, M.; Houshyar, Z. Influences of urea on preparation of zinc oxide nanostructures through chemical precipitation in ammonium hydrogencarbonate solution. Synthesis and Reactivity in Inorganic, Metal-Organic, and Nano-Metal Chemistry 2012, 42, 1363-1368.

[20] Wang, Y.; Zhang, C.; Bi, S.; Luo, G. Preparation of zno nanoparticles using the direct precipitation method in a membrane dispersion micro-structured reactor. Powder Technology 2010, 202, 130-136.

[21] Lanje, A. S.; Sharma, S. J.; Ningthoujam, R. S.; Ahn, J. S.; Pode, R. B. Low temperature dielectric studies of zinc oxide (zno) nanoparticles prepared by precipitation method. Adv. Powder Technol. 2013, 24, 331-335. 
[22] Niederberger, M.; Garnweitner, G.; Buha, J.; Polleux, J.; Ba, J.; Pinna, N. Nonaqueous synthesis of metal oxide nanoparticles:Review and indium oxide as case study for the dependence of particle morphology on precursors and solvents. J. Sol-Gel Sci. Technol. 2006, 40, 259-266.

[23] Lin, K.-F.; Cheng, H.-M.; Hsu, H.-C.; Lin, L.-J.; Hsieh, W.-F. Band gap variation of size-controlled zno quantum dots synthesized by sol-gel method. Chemical Physics Letters 2005, 409, 208-211.

[24] Hayat, K.; Gondal, M.; Khaled, M. M.; Ahmed, S.; Shemsi, A. M. Nano zno synthesis by modified sol gel method and its application in heterogeneous photocatalytic removal of phenol from water. Applied Catalysis A: General 2011, 393, 122-129.

[25] Yue, S.; Yan, Z.; Shi, Y.; Ran, G. Synthesis of zinc oxide nanotubes within ultrathin anodic aluminum oxide membrane by sol-gel method. Materials Letters 2013, 98, 246-249.

[26] Ristić, M.; Musić, S.; Ivanda, M.; Popović, S. Sol-gel synthesis and characterization of nanocrystalline zno powders. Journal of Alloys and Compounds 2005, 397, L1-L4.

[27] Benhebal, H.; Chaib, M.; Salmon, T.; Geens, J.; Leonard, A.; Lambert, S. D.; Crine, M.; Heinrichs, B. Photocatalytic degradation of phenol and benzoic acid using zinc oxide powders prepared by the sol-gel process. Alexandria Engineering Journal 2013, 52, 517-523.

[28] Meulenkamp, E. A. Synthesis and growth of zno nanoparticles. The Journal of Physical Chemistry B 1998, 102, 5566-5572.

[29] Lee, S.; Jeong, S.; Kim, D.; Hwang, S.; Jeon, M.; Moon, J. Zno nanoparticles with controlled shapes and sizes prepared using a simple polyol synthesis. Superlattices and Microstructures 2008, 43, 330-339.

[30] Caruntu, D.; Remond, Y.; Chou, N. H.; Jun, M.-J.; Caruntu, G.; He, J.; Goloverda, G.; O'Connor, C.; Kolesnichenko, V. Reactivity of 3d transition metal cations in diethylene glycol solutions. Synthesis of transition metal ferrites with the structure of discrete nanoparticles complexed with long-chain carboxylate anions. Inorganic Chemistry 2002, 41, 6137-6146.

[31] Ismail, A.; El-Midany, A.; Abdel-Aal, E.; El-Shall, H. Application of statistical design to optimize the preparation of zno nanoparticles via hydrothermal technique. Materials Letters 2005, 59, 1924-1928. 
[32] Chen, S.-J.; Li, L.-H.; Chen, X.-T.; Xue, Z.; Hong, J.-M.; You, X.-Z. Preparation and characterization of nanocrystalline zinc oxide by a novel solvothermal oxidation route. Journal of Crystal Growth 2003, 252, 184-189.

[33] Dem'Yanets, L.; Li, L.; Uvarova, T. Zinc oxide: Hydrothermal growth of nano-and bulk crystals and their luminescent properties. Journal of materials science 2006, $41,1439-1444$.

[34] Chen, D.; Jiao, X.; Cheng, G. Hydrothermal synthesis of zinc oxide powders with different morphologies. Solid State Communications 1999, 113, 363-366.

[35] Li, X.; He, G.; Xiao, G.; Liu, H.; Wang, M. Synthesis and morphology control of zno nanostructures in microemulsions. Journal of colloid and interface science 2009, 333, 465-473.

[36] Singhai, M.; Chhabra, V.; Kang, P.; Shah, D. Synthesis of zno nanoparticles for varistor application using zn-substituted aerosol ot microemulsion. Materials Research Bulletin 1997, 32, 239-247.

[37] Yıldırım, Ö. A.; Durucan, C. Synthesis of zinc oxide nanoparticles elaborated by microemulsion method. Journal of Alloys and Compounds 2010, 506, 944-949.

[38] Tang, X.; Choo, E. S. G.; Li, L.; Ding, J.; Xue, J. One-pot synthesis of water-stable zno nanoparticles via a polyol hydrolysis route and their cell labeling applications. Langmuir 2009, 25, 5271-5275.

[39] Becker, J.; Raghupathi, K. R.; St. Pierre, J.; Zhao, D.; Koodali, R. T. Tuning of the crystallite and particle sizes of zno nanocrystalline materials in solvothermal synthesis and their photocatalytic activity for dye degradation. The Journal of Physical Chemistry C 2011, 115, 13844-13850.

[40] Espitia, P. J. P.; Soares, N. d. F. F.; dos Reis Coimbra, J. S.; de Andrade, N. J.; Cruz, R. S.; Medeiros, E. A. A. Zinc oxide nanoparticles: Synthesis, antimicrobial activity and food packaging applications. Food and Bioprocess Technology 2012, $5,1447-1464$.

[41] Gunalan, S.; Sivaraj, R.; Rajendran, V. Green synthesized zno nanoparticles against bacterial and fungal pathogens. Progress in Natural Science: Materials International 2012, 22, 693-700.

[42] Talebian, N.; Amininezhad, S. M.; Doudi, M. Controllable synthesis of zno nanoparticles and their morphology-dependent antibacterial and optical properties. Journal of Photochemistry and Photobiology B: Biology 2013, 120, 66-73. 
[43] Punnoose, A.; Dodge, K.; Rasmussen, J. W.; Chess, J.; Wingett, D.; Anders, C. Cytotoxicity of zno nanoparticles can be tailored by modifying their surface structure: A green chemistry approach for safer nanomaterials. Acs Sustainable Chemistry \& Engineering 2014, 2, 1666-1673.

[44] Ischenko, V.; Polarz, S.; Grote, D.; Stavarache, V.; Fink, K.; Driess, M. Zinc oxide nanoparticles with defects. Advanced Functional Materials 2005, 15, 1945-1954.

[45] Naveed Ul Haq, A.; Nadhman, A.; Ullah, I.; Mustafa, G.; Yasinzai, M.; Khan, I. Synthesis approaches of zinc oxide nanoparticles: The dilemma of ecotoxicity. Journal of Nanomaterials 2017, 1-14.

[46] Chang, H.; Tsai, M.-H. Synthesis and characterization of zno nanoparticles having prism shape by a novel gas condensation process. Rev. Adv. Mater. Sci 2008, 18, 734-743.

[47] Pratsinis, S. E. Flame aerosol synthesis of ceramic powders. Progress in Energy and Combustion Science 1998, 24, 197-219.

[48] Pratsinis, S. E.; Vemury, S. Particle formation in gases: A review. Powder Technology 1996, 88, 267-273.

[49] Demokritou, P.; Buchel, R.; Molina, R. M.; Deloid, G. M.; Brain, J. D.; Pratsinis, S. E. Development and characterization of a versatile engineered nanomaterial generation system (venges) suitable for toxicological studies. Inhalation Toxicology 2010, 22, 107-116.

[50] Mueller, R.; Madler, L.; Pratsinis, S. E. Nanoparticle synthesis at high production rates by flame spray pyrolysis. Chemical Engineering Science 2003, 58, 19691976.

[51] Wegner, K.; Pratsinis, S. E. Scale-up of nanoparticle synthesis in diffusion flame reactors. Chemical Engineering Science 2003, 58, 4581-4589.

[52] Pratsinis, S. E. Aerosol-based technologies in nanoscale manufacturing: From functional materials to devices through core chemical engineering. Aiche Journal 2010, 56, 3028-3035.

[53] Gass, S.; Cohen, J. M.; Pyrgiotakis, G.; Sotiriou, G. A.; Pratsinis, S. E.; Demokritou, P. Safer formulation concept for flame-generated engineered nanomaterials. Acs Sustainable Chemistry \& Engineering 2013, 1, 843-857. 
[54] Sotiriou, G. A.; Watson, C.; Murdaugh, K. M.; Darrah, T. H.; Pyrgiotakis, G.; Elder, A.; Brain, J. D.; Demokritou, P. Engineering safer-by-design silica-coated zno nanorods with reduced DNA damage potential. Environmental Science-Nano 2014, 1, 144-153.

[55] Liqiang, J.; Yichun, Q.; Baiqi, W.; Shudan, L.; Baojiang, J.; Libin, Y.; Wei, F.; Honggang, F.; Jiazhong, S. Review of photoluminescence performance of nanosized semiconductor materials and its relationships with photocatalytic activity. Solar Energy Materials and Solar Cells 2006, 90, 1773-1787.

[56] Hidaka, H.; Horikoshi, S.; Serpone, N.; Knowland, J. In vitro photochemical damage to DNA, rna and their bases by an inorganic sunscreen agent on exposure to uva and uvb radiation. Journal of Photochemistry and Photobiology a-Chemistry 1997, 111, 205-213.

[57] Jyothi, K. P.; Yesodharan, S.; Yesodharan, E. P. Ultrasound (us), ultraviolet light (uv) and combination (us plus uv) assisted semiconductor catalysed degradation of organic pollutants in water: Oscillation in the concentration of hydrogen peroxide formed in situ. Ultrasonics Sonochemistry 2014, 21, 1787-1796.

[58] Bogdan, J.; Pławińska-Czarnak, J.; Zarzyńska, J. Nanoparticles of titanium and zinc oxides as novel agents in tumor treatment: A review. Nanoscale Research Letters 2017, 12, 1-15.

[59] Brenner, M. P.; Hilgenfeldt, S.; Lohse, D. Single-bubble sonoluminescence. Rev. Mod. Phys. 2002, 74, 425-484.

[60] Gupta, J.; Barick, K. C.; Bahadur, D. Defect mediated photocatalytic activity in shape-controlled zno nanostructures. Journal of Alloys and Compounds 2011, 509, 6725-6730.

[61] Saliani, M.; Jalal, R.; Goharshadi, E. K. Mechanism of oxidative stress involved in the toxicity of zno nanoparticles against eukaryotic cells. Nanomedicine Journal 2016, 3, 1-14.

[62] Guo, D.; Bi, H.; Liu, B.; Wu, Q.; Wang, D.; Cui, Y. Reactive oxygen speciesinduced cytotoxic effects of zinc oxide nanoparticles in rat retinal ganglion cells. Toxicology in Vitro 2013, 27, 731-738.

[63] Guo, M. Y.; Ng, A. M. C.; Liu, F.; Djurišić, A. B.; Chan, W. K. Photocatalytic activity of metal oxides - the role of holes and oh radicals. Applied Catalysis B: Environmental 2011, 107, 150-157. 
[64] Zheng, Y.; Chen, C.; Zhan, Y.; Lin, X.; Zheng, Q.; Wei, K.; Zhu, J.; Zhu, Y. Luminescence and photocatalytic activity of zno nanocrystals: Correlation between structure and property. Inorganic Chemistry 2007, 46, 6675-6682.

[65] Khan, M. F.; Ansari, A. H.; Hameedullah, M.; Ahmad, E.; Husain, F. M.; Zia, Q.; Baig, U.; Zaheer, M. R.; Alam, M. M.; Khan, A. M. et al. Sol-gel synthesis of thornlike zno nanoparticles endorsing mechanical stirring effect and their antimicrobial activities: Potential role as nano-antibiotics. Scientific Reports 2016, 6, 1-11.

[66] McLaren, A.; Valdes-Solis, T.; Li, G. Q.; Tsang, S. C. Shape and size effects of zno nanocrystals on photocatalytic activity. Journal of the American Chemical Society 2009, 131, 12540-12541.

[67] Nair, S.; Sasidharan, A.; Rani, V. D.; Menon, D.; Nair, S.; Manzoor, K.; Raina, S. Role of size scale of zno nanoparticles and microparticles on toxicity toward bacteria and osteoblast cancer cells. Journal of Materials Science: Materials in Medicine 2009, 20, S235-S241.

[68] Jang, Y. J.; Simer, C.; Ohm, T. Comparison of zinc oxide nanoparticles and its nano-crystalline particles on the photocatalytic degradation of methylene blue. Materials Research Bulletin 2006, 41, 67-77.

[69] Li, D.; Haneda, H. Morphologies of zinc oxide particles and their effects on photocatalysis. Chemosphere 2003, 51, 129-137.

[70] Li, M. H.; Pokhrel, S.; Jin, X.; Madler, L.; Damoiseaux, R.; Hoek, E. M. V. Stability, bioavailability, and bacterial toxicity of zno and iron-doped zno nanoparticles in aquatic media. Environmental Science \& Technology 2011, 45, $755-761$.

[71] Leroueil, P. R.; Berry, S. A.; Duthie, K.; Han, G.; Rotello, V. M.; McNerny, D. Q.; Baker, J. R.; Orr, B. G.; Banaszak Holl, M. M. Wide varieties of cationic nanoparticles induce defects in supported lipid bilayers. Nano Letters 2008, 8, 420424.

[72] Li, Y.; Gu, N. Thermodynamics of charged nanoparticle adsorption on chargeneutral membranes: A simulation study. J. Phys. Chem. B 2010, 114, 2749-2754.

[73] Verma, A.; Stellacci, F. Effect of surface properties on nanoparticle-cell interactions. Small 2010, 6, 12-21.

[74] Bhattacharjee, S.; de Haan, L. H. J.; Evers, N. M.; Jiang, X.; Marcelis, A. T. M.; Zuilhof, H.; Rietjens, I.; Alink, G. M. Role of surface charge and oxidative stress 
in cytotoxicity of organic monolayer-coated silicon nanoparticles towards macrophage nr8383 cells. Particle and Fibre Toxicology 2010, 7, 1-12.

[75] Arvizo, R. R.; Miranda, O. R.; Thompson, M. A.; Pabelick, C. M.; Bhattacharya, R.; Robertson, J. D.; Rotello, V. M.; Prakash, Y. S.; Mukherjee, P. Effect of nanoparticle surface charge at the plasma membrane and beyond. Nano Letters 2010, 10, 2543-2548.

[76] Leroueil, P. R.; Hong, S. Y.; Mecke, A.; Baker, J. R.; Orr, B. G.; Holl, M. M. B. Nanoparticle interaction with biological membranes: Does nanotechnology present a janus face? Accounts of Chemical Research 2007, 40, 335-342.

[77] Cho, E. C.; Xie, J. W.; Wurm, P. A.; Xia, Y. N. Understanding the role of surface charges in cellular adsorption versus internalization by selectively removing gold nanoparticles on the cell surface with a i-2/ki etchant. Nano Letters 2009, 9, 10801084 .

[78] Wingett, D.; Louka, P.; Anders, C. B.; Zhang, J. H.; Punnoose, A. A role of zno nanoparticle electrostatic properties in cancer cell cytotoxicity. Nanotechnology Science and Applications 2016, 9, 29-45.

[79] Sabuncu, A. C.; Grubbs, J.; Qian, S. Z.; Abdel-Fattah, T. M.; Stacey, M. W.; Beskok, A. Probing nanoparticle interactions in cell culture media. Colloids and Surfaces B-Biointerfaces 2012, 95, 96-102.

[80] Cho, E. C.; Zhang, Q.; Xia, Y. The effect of sedimentation and diffusion on cellular uptake of gold nanoparticles. Nature Nanotechnology 2011, 6, 385-391.

[81] Jassby, D.; Farner Budarz, J.; Wiesner, M. Impact of aggregate size and structure on the photocatalytic properties of tio2 and zno nanoparticles. Environ Sci Technol $2012,46,6934-41$.

[82] Horie, M.; Nishio, K.; Fujita, K.; Kato, H.; Endoh, S.; Suzuki, M.; Nakamura, A.; Miyauchi, A.; Kinugasa, S.; Yamamoto, K. et al. Cellular responses by stable and uniform ultrafine titanium dioxide particles in culture-medium dispersions when secondary particle size was $100 \mathrm{~nm}$ or less. Toxicol. Vitro 2010, 24, 1629-1638.

[83] Maiorano, G.; Sabella, S.; Sorce, B.; Brunetti, V.; Malvindi, M. A.; Cingolani, R.; Pompa, P. P. Effects of cell culture media on the dynamic formation of proteinnanoparticle complexes and influence on the cellular response. Acs Nano 2010, 4, 7481-7491. 
[84] Setyawati, M. I.; Tay, C. Y.; Chia, S. L.; Goh, S. L.; Fang, W.; Neo, M. J.; Chong, H. C.; Tan, S. M.; Loo, S. C. J.; Ng, K. W. et al. Titanium dioxide nanomaterials cause endothelial cell leakiness by disrupting the homophilic interaction of vecadherin. Nature Communications 2013, 4, 1-12.

[85] Setyawati, M. I.; Tay, C. Y.; Docter, D.; Stauber, R. H.; Leong, D. T. Understanding and exploiting nanoparticles' intimacy with the blood vessel and blood. Chemical Society Reviews 2015, 8174-8199.

[86] Brayner, R.; Dahoumane, S. A.; Yepremian, C.; Djediat, C.; Meyer, M.; Coute, A.; Fievet, F. Zno nanoparticles: Synthesis, characterization, and ecotoxicological studies. Langmuir 2010, 26, 6522-6528.

[87] Wells, M. A.; Abid, A.; Kennedy, I. M.; Barakat, A. I. Serum proteins prevent aggregation of fe2o3 and zno nanoparticles. Nanotoxicology 2012, 6, 837-846.

[88] Xu, M.; Li, J.; Iwai, H.; Mei, Q.; Fujita, D.; Su, H.; Chen, H.; Hanagata, N. Formation of nano-bio-complex as nanomaterials dispersed in a biological solution for understanding nanobiological interactions. Sci Rep 2012, 2, 1-6.

[89] Tenzer, S.; Docter, D.; Kuharev, J.; Musyanovych, A.; Fetz, V.; Hecht, R.; Schlenk, F.; Fischer, D.; Kiouptsi, K.; Reinhardt, C. et al. Rapid formation of plasma protein corona critically affects nanoparticle pathophysiology. Nature Nanotechnology 2013, 8, 772-781.

[90] Monopoli, M. P.; Aberg, C.; Salvati, A.; Dawson, K. A. Biomolecular coronas provide the biological identity of nanosized materials. Nature Nanotechnology 2012, 7, 779-786.

[91] Keller, A. A.; Wang, H.; Zhou, D.; Lenihan, H. S.; Cherr, G.; Cardinale, B. J.; Miller, R.; Ji, Z. Stability and aggregation of metal oxide nanoparticles in natural aqueous matrices. Environmental Science \& Technology 2010, 44, 1962-1967.

[92] Lesniak, A.; Fenaroli, F.; Monopoli, M. R.; Aberg, C.; Dawson, K. A.; Salvati, A. Effects of the presence or absence of a protein corona on silica nanoparticle uptake and impact on cells. ACS Nano 2012, 6, 5845-5857.

[93] Deng, Z. J.; Mortimer, G.; Schiller, T.; Musumeci, A.; Martin, D.; Minchin, R. F. Differential plasma protein binding to metal oxide nanoparticles. Nanotechnology 2009, 20, 1-9.

[94] Bihari, P.; Vippola, M.; Schultes, S.; Praetner, M.; Khandoga, A. G.; Reichel, C. A.; Coester, C.; Tuomi, T.; Rehberg, M.; Krombach, F. Optimized dispersion of 
nanoparticles for biological in vitro and in vivo studies. Part Fibre Toxicol 2008, 5, $1-14$.

[95] Misra, S. K.; Dybowska, A.; Berhanu, D.; Luoma, S. N.; Valsami-Jones, E. The complexity of nanoparticle dissolution and its importance in nanotoxicological studies. Sci. Total Environ. 2012, 438, 225-232.

[96] Bian, S.-W.; Mudunkotuwa, I. A.; Rupasinghe, T.; Grassian, V. H. Aggregation and dissolution of $4 \mathrm{~nm}$ zno nanoparticles in aqueous environments: Influence of ph, ionic strength, size, and adsorption of humic acid. Langmuir 2011, 27, 60596068.

[97] Li, M.; Zhu, L.; Lin, D. Toxicity of zno nanoparticles to escherichia coli: Mechanism and the influence of medium components. Environ Sci Technol 2011, 45, 1977-1983.

[98] Xia, T.; Kovochich, M.; Liong, M.; Madler, L.; Gilbert, B.; Shi, H. B.; Yeh, J. I.; Zink, J. I.; Nel, A. E. Comparison of the mechanism of toxicity of zinc oxide and cerium oxide nanoparticles based on dissolution and oxidative stress properties. ACS Nano 2008, 2, 2121-2134.

[99] Eixenberger, J.; Anders, C.; Hermann, R.; Brown, R.; Reddy, K. M.; Punnoose, A.; Wingett, D. Rapid dissolution of zno nanoparticles induced by biological buffers significantly impacts cytotoxicity. Chemical Research in Toxicology 2017, 30, 1641-1651.

[100] Reed, R. B.; Ladner, D. A.; Higgins, C. P.; Westerhoff, P.; Ranville, J. F. Solubility of nano-zinc oxide in environmentally and biologically important matrices. Environmental Toxicology and Chemistry 2012, 31, 93-99.

[101] Lanzl, C. A.; Baltrusaitis, J.; Cwiertny, D. M. Dissolution of hematite nanoparticle aggregates: Influence of primary particle size, dissolution mechanism, and solution ph. Langmuir 2012, 28, 15797-808.

[102] Muller, K. H.; Kulkarni, J.; Motskin, M.; Goode, A.; Winship, P.; Skepper, J. N.; Ryan, M. P.; Porter, A. E. Ph-dependent toxicity of high aspect ratio zno nanowires in macrophages due to intracellular dissolution. ACS Nano 2010, 4, 6767-6779.

[103] Yin, H.; Casey, P. S. Effects of iron or manganese doping of zno nanoparticles on their dissolution, ros generation and cytotoxicity. Rsc Advances 2014, 4, 2614926157. 
[104] Baek, M.; Kim, M. K.; Cho, H. J.; Lee, J. A.; Yu, J.; Chung, H. E.; Choi, S. J. Factors influencing the cytotoxicity of zinc oxide nanoparticles: Particle size and surface charge. Journal of Physics: Conference Series 2011, 304, 1-7.

[105] David, C. A.; Galceran, J.; Rey-Castro, C.; Puy, J.; Companys, E.; Salvador, J.; Monné, J.; Wallace, R.; Vakourov, A. Dissolution kinetics and solubility of zno nanoparticles followed by agnes. The Journal of Physical Chemistry C 2012, 116, 11758-11767.

[106] Song, W. H.; Zhang, J. Y.; Guo, J.; Zhang, J. H.; Ding, F.; Li, L. Y.; Sun, Z. T. Role of the dissolved zinc ion and reactive oxygen species in cytotoxicity of zno nanoparticles. Toxicology Letters 2010, 199, 389-397.

[107] Turney, T. W.; Duriska, M. B.; Jayaratne, V.; Elbaz, A.; O'Keefe, S. J.; Hastings, A. S.; Piva, T. J.; Wright, P. F. A.; Feltis, B. N. Formation of zinc-containing nanoparticles from $\mathrm{zn} 2+$ ions in cell culture media: Implications for the nanotoxicology of zno. Chemical Research in Toxicology 2012, 25, 2057-2066.

[108] Amara, S.; Ben Slama, I.; Mrad, I.; Rihane, N.; Khemissi, W.; El Mir, L.; Ben Rhouma, K.; Abdelmelek, H.; Sakly, M. Effects of zinc oxide nanoparticles and/or zinc chloride on biochemical parameters and mineral levels in rat liver and kidney. Human \& Experimental Toxicology 2014, 33, 1150-1157.

[109] Wahab, R.; Ansari, S. G.; Kim, Y. S.; Dar, M. A.; Shin, H. S. Synthesis and characterization of hydrozincite and its conversion into zinc oxide nanoparticles. Journal of Alloys and Compounds 2008, 461, 66-71.

[110] Mu, Q. S.; David, C. A.; Galceran, J.; Rey-Castro, C.; Krzeminski, L.; Wallace, R.; Bamiduro, F.; Milne, S. J.; Hondow, N. S.; Brydson, R. et al. Systematic investigation of the physicochemical factors that contribute to the toxicity of zno nanoparticles. Chemical Research in Toxicology 2014, 27, 558-567.

[111] Lv, J.; Zhang, S.; Luo, L.; Han, W.; Zhang, J.; Yang, K.; Christie, P. Dissolution and microstructural transformation of zno nanoparticles under the influence of phosphate. Environmental Science \& Technology 2012, 46, 7215-7221.

[112] Saptarshi, S. R.; Duschl, A.; Lopata, A. L. Biological reactivity of zinc oxide nanoparticles with mammalian test systems: An overview. Nanomedicine 2015, 10, 2075-2092.

[113] Pattan, G.; Kaul, G. Health hazards associated with nanomaterials. Toxicology and Industrial Health 2014, 30, 499-519. 
[114] Wang, B.; Feng, W.; Wang, M.; Wang, T.; Gu, Y.; Zhu, M.; Ouyang, H.; Shi, J.; Zhang, F.; Zhao, Y. et al. Acute toxicological impact of nano- and submicro-scaled zinc oxide powder on healthy adult mice. Journal of Nanoparticle Research 2008, $10,263-276$.

[115] Akhtar, M. J.; Ahamed, M.; Kumar, S.; Khan, M. M.; Ahmad, J.; Alrokayan, S. A. Zinc oxide nanoparticles selectively induce apoptosis in human cancer cells through reactive oxygen species. Int J Nanomedicine 2012, 7, 845-857.

[116] Bhattacharya, D.; Santra, C. R.; Ghosh, A. N.; Karmakar, P. Differential toxicity of rod and spherical zinc oxide nanoparticles on human peripheral blood mononuclear cells. Journal of Biomedical Nanotechnology 2014, 10, 707-716.

[117] Condello, M.; De Berardis, B.; Ammendolia, M. G.; Barone, F.; Condello, G.; Degan, P.; Meschini, S. Zno nanoparticle tracking from uptake to genotoxic damage in human colon carcinoma cells. Toxicology in Vitro 2016, 35, 169-179.

[118] Gilbert, B.; Fakra, S. C.; Xia, T.; Pokhrel, S.; Madler, L.; Nel, A. E. The fate of zno nanoparticles administered to human bronchial epithelial cells. ACS Nano 2012, 6, 4921-4930.

[119] Hsiao, I. L.; Huang, Y. J. Effects of various physicochemical characteristics on the toxicities of zno and tio2 nanoparticles toward human lung epithelial cells. Sci. Total Environ. 2011, 409, 1219-1228.

[120] Malugin, A.; Herd, H.; Ghandehari, H. Differential toxicity of amorphous silica nanoparticles toward phagocytic and epithelial cells. Journal of Nanoparticle Research 2011, 13, 5381-5396.

[121] Ramasamy, M.; Das, M.; An, S. S. A.; Yi, D. K. Role of surface modification in zinc oxide nanoparticles and its toxicity assessment toward human dermal fibroblast cells. International Journal of Nanomedicine 2014, 9, 3707-3718.

[122] Zvyagin, A. V.; Zhao, X.; Gierden, A.; Sanchez, W.; Ross, J. A.; Roberts, M. S. Imaging of zinc oxide nanoparticle penetration in human skin in vitro and in vivo. Journal of biomedical optics 2008, 13, 0640311-0640319.

[123] Jeng, H. A.; Swanson, J. Toxicity of metal oxide nanoparticles in mammalian cells. J. Environ. Sci. Health Part A-Toxic/Hazard. Subst. Environ. Eng. 2006, 41, 2699 2711. 
[124] Reddy, K. M.; Feris, K.; Bell, J.; Wingett, D. G.; Hanley, C.; Punnoose, A. Selective toxicity of zinc oxide nanoparticles to prokaryotic and eukaryotic systems. Applied Physics Letters 2007, 90, 1-8.

[125] Choi, J. S.; Kim, R.-O.; Yoon, S.; Kim, W.-K. Developmental toxicity of zinc oxide nanoparticles to zebrafish (danio rerio): A transcriptomic analysis. PloS one 2016, 11, 1-15.

[126] Wehmas, L. C.; Anders, C.; Chess, J.; Punnoose, A.; Pereira, C. B.; Greenwood, J. A.; Tanguay, R. L. Comparative metal oxide nanoparticle toxicity using embryonic zebrafish. Toxicology reports 2015, 2, 702-715.

[127] Zhang, W.; Bao, S.; Fang, T. The neglected nano-specific toxicity of zno nanoparticles in the yeast saccharomyces cerevisiae. Scientific reports 2016, 6, 111.

[128] Kasemets, K.; Ivask, A.; Dubourguier, H.-C.; Kahru, A. Toxicity of nanoparticles of zno, cuo and tio2 to yeast saccharomyces cerevisiae. Toxicology in Vitro 2009, 23, 1116-1122.

[129] Wang, H.; Wick, R. L.; Xing, B. Toxicity of nanoparticulate and bulk zno, al2o3 and tio2 to the nematode caenorhabditis elegans. Environmental Pollution 2009, $157,1171-1177$.

[130] Aruoja, V.; Dubourguier, H.-C.; Kasemets, K.; Kahru, A. Toxicity of nanoparticles of cuo, zno and tio2 to microalgae pseudokirchneriella subcapitata. Sci. Total Environ. 2009, 407, 1461-1468.

[131] Franklin, N. M.; Rogers, N. J.; Apte, S. C.; Batley, G. E.; Gadd, G. E.; Casey, P. S. Comparative toxicity of nanoparticulate zno, bulk zno, and zncl2 to a freshwater microalga (pseudokirchneriella subcapitata): The importance of particle solubility. Environmental Science \& Technology 2007, 41, 8484-8490.

[132] Heinlaan, M.; Ivask, A.; Blinova, I.; Dubourguier, H.-C.; Kahru, A. Toxicity of nanosized and bulk zno, cuo and tio2 to bacteria vibrio fischeri and crustaceans daphnia magna and thamnocephalus platyurus. Chemosphere 2008, 71, 1308-1316.

[133] Huang, C.-C.; Aronstam, R. S.; Chen, D.-R.; Huang, Y.-W. Oxidative stress, calcium homeostasis, and altered gene expression in human lung epithelial cells exposed to zno nanoparticles. Toxicology in Vitro 2010, 24, 45-55. 
[134] Sharma, V.; Anderson, D.; Dhawan, A. Zinc oxide nanoparticles induce oxidative DNA damage and ros-triggered mitochondria mediated apoptosis in human liver cells (hepg2). Apoptosis 2012, 17, 852-870.

[135] Xia, T.; Kovochich, M.; Liong, M.; Mädler, L.; Gilbert, B.; Shi, H.; Yeh, J.; Zink, J.; Nel, A. Comparison of the mechanism of toxicity of zinc oxide and cerium oxide nanoparticles based on dissolution and oxidative stress properties. ACS Nano 2008, 2, 2121-2134.

[136] Yang, H.; Liu, C.; Yang, D.; Zhang, H.; Xi, Z. Comparative study of cytotoxicity, oxidative stress and genotoxicity induced by four typical nanomaterials: The role of particle size, shape and composition. Journal of applied Toxicology 2009, 29, 69-78.

[137] Xu, M.; Fujita, D.; Kajiwara, S.; Minowa, T.; Li, X.; Takemura, T.; Iwai, H.; Hanagata, N. Contribution of physicochemical characteristics of nano-oxides to cytotoxicity. Biomaterials 2010, 31, 8022-8031.

[138] Bozym, R. A.; Chimienti, F.; Giblin, L. J.; Gross, G. W.; Korichneva, I.; Li, Y. A.; Libert, S.; Maret, W.; Parviz, M.; Frederickson, C. J. et al. Free zinc ions outside a narrow concentration range are toxic to a variety of cells in vitro. Experimental Biology and Medicine 2010, 235, 741-750.

[139] Kao, Y. Y.; Chen, Y. C.; Cheng, T. J.; Chiung, Y. M.; Liu, P. S. Zinc oxide nanoparticles interfere with zinc ion homeostasis to cause cytotoxicity. Toxicological Sciences 2012, 125, 462-472.

[140] Othman, B. A.; Greenwood, C.; Abuelela, A. F.; Bharath, A. A.; Chen, S.; Theodorou, I.; Douglas, T.; Uchida, M.; Ryan, M.; Merzaban, J. S. et al. Correlative light-electron microscopy shows rgd-targeted zno nanoparticles dissolve in the intracellular environment of triple negative breast cancer cells and cause apoptosis with intratumor heterogeneity. Advanced Healthcare Materials 2016, 5, 1310-1325.

[141] Wang, B.; Zhang, Y. Y.; Mao, Z. W.; Yu, D. H.; Gao, C. Y. Toxicity of zno nanoparticles to macrophages due to cell uptake and intracellular release of zinc ions. Journal of nanoscience and nanotechnology 2014, 14, 5688-5696.

[142] Park, S. J.; Park, Y. C.; Lee, S. W.; Jeong, M. S.; Yu, K.-N.; Jung, H.; Lee, J.-K.; Kim, J. S.; Cho, M.-H. Comparing the toxic mechanism of synthesized zinc oxide nanomaterials by physicochemical characterization and reactive oxygen species properties. Toxicology Letters 2011, 207, 197-203. 
[143] Thongkam, W.; Gerloff, K.; van Berlo, D.; Albrecht, C.; Schins, R. P. F. Oxidant generation, DNA damage and cytotoxicity by a panel of engineered nanomaterials in three different human epithelial cell lines. Mutagenesis 2017, 32, 105-115.

[144] Bienert, G. P.; Schjoerring, J. K.; Jahn, T. P. Membrane transport of hydrogen peroxide. Biochimica et Biophysica Acta (BBA) - Biomembranes 2006, 1758, 9941003.

[145] Yu, K. N.; Yoon, T. J.; Minai-Tehrani, A.; Kim, J. E.; Park, S. J.; Jeong, M. S.; Ha, S. W.; Lee, J. K.; Kim, J. S.; Cho, M. H. Zinc oxide nanoparticle induced autophagic cell death and mitochondrial damage via reactive oxygen species generation. Toxicology in Vitro 2013, 27, 1187-1195.

[146] Sasidharan, A.; Chandran, P.; Menon, D.; Raman, S.; Nair, S.; Koyakutty, M. Rapid dissolution of zno nanocrystals in acidic cancer microenvironment leading to preferential apoptosis. Nanoscale 2011, 3, 3657-3669.

[147] Toduka, Y.; Toyooka, T.; Ibuki, Y. Flow cytometric evaluation of nanoparticles using side-scattered light and reactive oxygen species-mediated fluorescencecorrelation with genotoxicity. Environmental Science \& Technology 2012, 46, 7629-7636.

[148] Yang, H.; Liu, C.; Yang, D. F.; Zhang, H. S.; Xi, Z. G. Comparative study of cytotoxicity, oxidative stress and genotoxicity induced by four typical nanomaterials: The role of particle size, shape and composition. Journal of Applied Toxicology 2009, 29, 69-78.

[149] Abercrombie, M.; Ambrose, E. J. The surface properties of cancer cells: A review. Cancer research 1962, 22, 525-548.

[150] Panchal, R. G. Novel therapeutic strategies to selectively kill cancer cells. Biochemical Pharmacology 1998, 55, 247-252.

[151] Papo, N.; Shahar, M.; Eisenbach, L.; Shai, Y. A novel lytic peptide composed of dl-amino acids selectively kills cancer cells in culture and in mice. Journal of Biological Chemistry 2003, 278, 21018-21023.

[152] Shen, C. C.; James, S. A.; de Jonge, M. D.; Turney, T. W.; Wright, P. F. A.; Feltis, B. N. Relating cytotoxicity, zinc ions, and reactive oxygen in zno nanoparticleexposed human immune cells. Toxicological Sciences 2013, 136, 120130. 
[153] Hong, T. K.; Tripathy, N.; Son, H. J.; Ha, K. T.; Jeong, H. S.; Hahn, Y. B. A comprehensive in vitro and in vivo study of zno nanoparticles toxicity. Journal of Materials Chemistry B 2013, 1, 2985-2992.

[154] Krezel, A.; Maret, W. Zinc-buffering capacity of a eukaryotic cell at physiological pzn. Journal of Biological Inorganic Chemistry 2006, 11, 1049-1062.

[155] Rice, J. M.; Zweifach, A.; Lynes, M. A. Metallothionein regulates intracellular zinc signaling during cd4(+) t cell activation. Bmc Immunology 2016, 17, 1-14.

[156] Andrews, G. K. Regulation of metallothionein gene expression by oxidative stress and metal ions. Biochemical Pharmacology 2000, 59, 95-104.

[157] Liuzzi, J. P.; Cousins, R. J. Mammalian zinc transporters. Annu. Rev. Nutr. 2004, 24, 151-172.

[158] Sullivan, V. K.; Burnett, F. R.; Cousins, R. J. Metallothionein expression is increased in monocytes and erythrocytes of young men during zinc supplementation. The Journal of nutrition 1998, 128, 707-713.

[159] Sutherland, D. E.; Stillman, M. J. The "magic numbers" of metallothionein. Metallomics 2011, 3, 444-463.

[160] Li, J. H.; Liu, X. R.; Zhang, Y.; Tian, F. F.; Zhao, G. Y.; Yu, Q. L. Y.; Jiang, F. L.; Liu, Y. Toxicity of nano zinc oxide to mitochondria. Toxicology Research 2012, 1 , 137-144.

[161] Zhang, J. X.; Wang, X. L.; Vikash, V.; Ye, Q.; Wu, D. D.; Liu, Y. L.; Dong, W. G. Ros and ros-mediated cellular signaling. Oxidative Med. Cell. Longev. 2016, 1-18.

[162] Jomova, K.; Valko, M. Advances in metal-induced oxidative stress and human disease. Toxicology 2011, 283, 65-87.

[163] Rink, L.; Haase, H. Zinc homeostasis and immunity. Trends in Immunology 2007, $28,1-4$.

[164] Colvin, R. A.; Holmes, W. R.; Fontaine, C. P.; Maret, W. Cytosolic zinc buffering and muffling: Their role in intracellular zinc homeostasis. Metallomics 2010, 2, 306-317.

[165] Maret, W. Analyzing free zinc(ii) ion concentrations in cell biology with fluorescent chelating molecules. Metallomics 2015, 7, 202-211. 
[166] Haase, H.; Rink, L. Functional significance of zinc-related signaling pathways in immune cells. Annual Review of Nutrition 2009, 29, 133-152.

[167] Andrews, G. K. Cellular zinc sensors: Mtf-1 regulation of gene expression. Biometals 2001, 14, 223-237.

[168] Laity, J. H.; Andrews, G. K. Understanding the mechanisms of zinc-sensing by metal-response element binding transcription factor-1 (mtf-1). Archives of Biochemistry and Biophysics 2007, 463, 201-210.

[169] Palmiter, R. D. Protection against zinc toxicity by metallothionein and zinc transporter 1. Proceedings of the National Academy of Sciences of the United States of America 2004, 101, 4918-4923.

[170] Maret, W. The function of zinc metallothionein: A link between cellular zinc and redox state. The Journal of Nutrition 2000, 130, 1455S-1458S.

[171] Waldron, K. J.; Rutherford, J. C.; Ford, D.; Robinson, N. J. Metalloproteins and metal sensing. Nature 2009, 460, 823-830.

[172] Jeong, J.; Eide, D. J. The slc39 family of zinc transporters. Molecular Aspects of Medicine 2013, 34, 612-619.

[173] Overbeck, S.; Uciechowski, P.; Ackland, M. L.; Ford, D.; Rink, L. Intracellular zinc homeostasis in leukocyte subsets is regulated by different expression of zinc exporters znt-1 to znt-9. Journal of Leukocyte Biology 2008, 83, 368-380.

[174] Hirano, T.; Murakami, M.; Fukada, T.; Nishida, K.; Yamasaki, S.; Suzuki, T. Roles of zinc and zinc signaling in immunity: Zinc as an intracellular signaling molecule. In Advances in immunology, vol 97. Alt, F. W.; Austen, K. F.; Honjo, T.; Melchers, F.; Uhr, J. W.; Unanue, E. R., Eds.; 2008; pp 149-176.

[175] Yamasaki, S.; Sakata-Sogawa, K.; Hasegawa, A.; Suzuki, T.; Kabu, K.; Sato, E.; Kurosaki, T.; Yamashita, S.; Tokunaga, M.; Nishida, K. et al. Zinc is a novel intracellular second messenger. Journal of Cell Biology 2007, 177, 637-645.

[176] Yamasaki, S.; Hasegawa, A.; Hojyo, S.; Ohashi, W.; Fukada, T.; Nishida, K.; Hirano, T. A novel role of the l-type calcium channel alpha(1d) subunit as a gatekeeper for intracellular zinc signaling: Zinc wave. PloS one 2012, 7, 1-7.

[177] Yu, M. C.; Lee, W. W.; Tomar, D.; Pryshchep, S.; Czesnikiewicz-Guzik, M.; Lamar, D. L.; Li, G. J.; Singh, K.; Tian, L.; Weyand, C. M. et al. Regulation of t 
cell receptor signaling by activation-induced zinc influx. Journal of Experimental Medicine 2011, 208, 775-785.

[178] Kitamura, H.; Morikawa, H.; Kamon, H.; Iguchi, M.; Hojyo, S.; Fukada, T.; Yamashita, S.; Kaisho, T.; Akira, S.; Murakami, M. et al. Toll-like receptormediated regulation of zinc homeostasis influences dendritic cell function. Nature Immunology 2006, 7, 971-977.

[179] Aydemir, T. B.; Blanchard, R. K.; Cousins, R. J. Zinc supplementation of young men alters metallothionein, zinc transporter, and cytokine gene expression in leukocyte populations. Proceedings of the National Academy of Sciences of the United States of America 2006, 103, 1699-1704.

[180] Lin, S.-f.; Wei, H.; Maeder, D.; Franklin, R. B.; Feng, P. Profiling of zinc-altered gene expression in human prostate normal vs. Cancer cells: A time course study. The Journal of nutritional biochemistry 2009, 20, 1000-1012.

[181] Moos, P. J.; Olszewski, K.; Honeggar, M.; Cassidy, P.; Leachman, S.; Woessner, D.; Cutler, N. S.; Veranth, J. M. Responses of human cells to zno nanoparticles: A gene transcription study. Metallomics 2011, 3, 1199-1211.

[182] Triboulet, S.; Aude-Garcia, C.; Armand, L.; Gerdil, A.; Diemer, H.; Proamer, F.; Collin-Faure, V.; Habert, A.; Strub, J. M.; Hanau, D. et al. Analysis of cellular responses of macrophages to zinc ions and zinc oxide nanoparticles: A combined targeted and proteomic approach. Nanoscale 2014, 6, 6102-6114.

[183] Tuomela, S.; Autio, R.; Buerki-Thurnherr, T.; Arslan, O.; Kunzmann, A.; Andersson-Willman, B.; Wick, P.; Mathur, S.; Scheynius, A.; Krug, H. F. et al. Gene expression profiling of immune-competent human cells exposed to engineered zinc oxide or titanium dioxide nanoparticles. PloS one 2013, 8, 1-18.

[184] Lee, S. H.; Pie, J. E.; Kim, Y. R.; Lee, H. R.; Son, S. W.; Kim, M. K. Effects of zinc oxide nanoparticles on gene expression profile in human keratinocytes. Molecular \& Cellular Toxicology 2012, 8, 113-118.

[185] Sullivan, V. K.; Cousins, R. J. Competitive reverse transcriptase-polymerase chain reaction shows that dietary zinc supplementation in humans increases monocyte metallothionein mrna levels. The Journal of nutrition 1997, 127, 694-698.

[186] Beyersmann, D.; Haase, H. Functions of zinc in signaling, proliferation and differentiation of mammalian cells. Biometals 2001, 14, 331-341. 
[187] Smith, G. D.; Swenson, D. C.; Dodson, E. J.; Dodson, G. G.; Reynolds, C. D. Structural stability in the 4-zinc human insulin hexamer. Proceedings of the National Academy of Sciences of the United States of America-Biological Sciences 1984, 81, 7093-7097.

[188] Editor, Join the dialogue. In Nat Nano, Nature Publishing Group, a division of Macmillan Publishers Limited. All Rights Reserved.: 2012; Vol. 7, pp 545-545.

[189] Schrurs, F.; Lison, D. Focusing the research effort. Nature Nanotechnology 2012, 7, 546-548.

[190] Landsiedel, R.; Ma-Hock, L.; Kroll, A.; Hahn, D.; Schnekenburger, J.; Wiench, K.; Wohlleben, W. Testing metal-oxide nanomaterials for human safety. Adv Mater 2010, 22, 2601-2627.

[191] Sambale, F.; Stahl, F.; Rudinger, F.; Seliktar, D.; Kasper, C.; Bahnemann, D.; Scheper, T. Iterative cellular screening system for nanoparticle safety testing. Journal of Nanomaterials 2015, 1-16. 
Tables and Figures

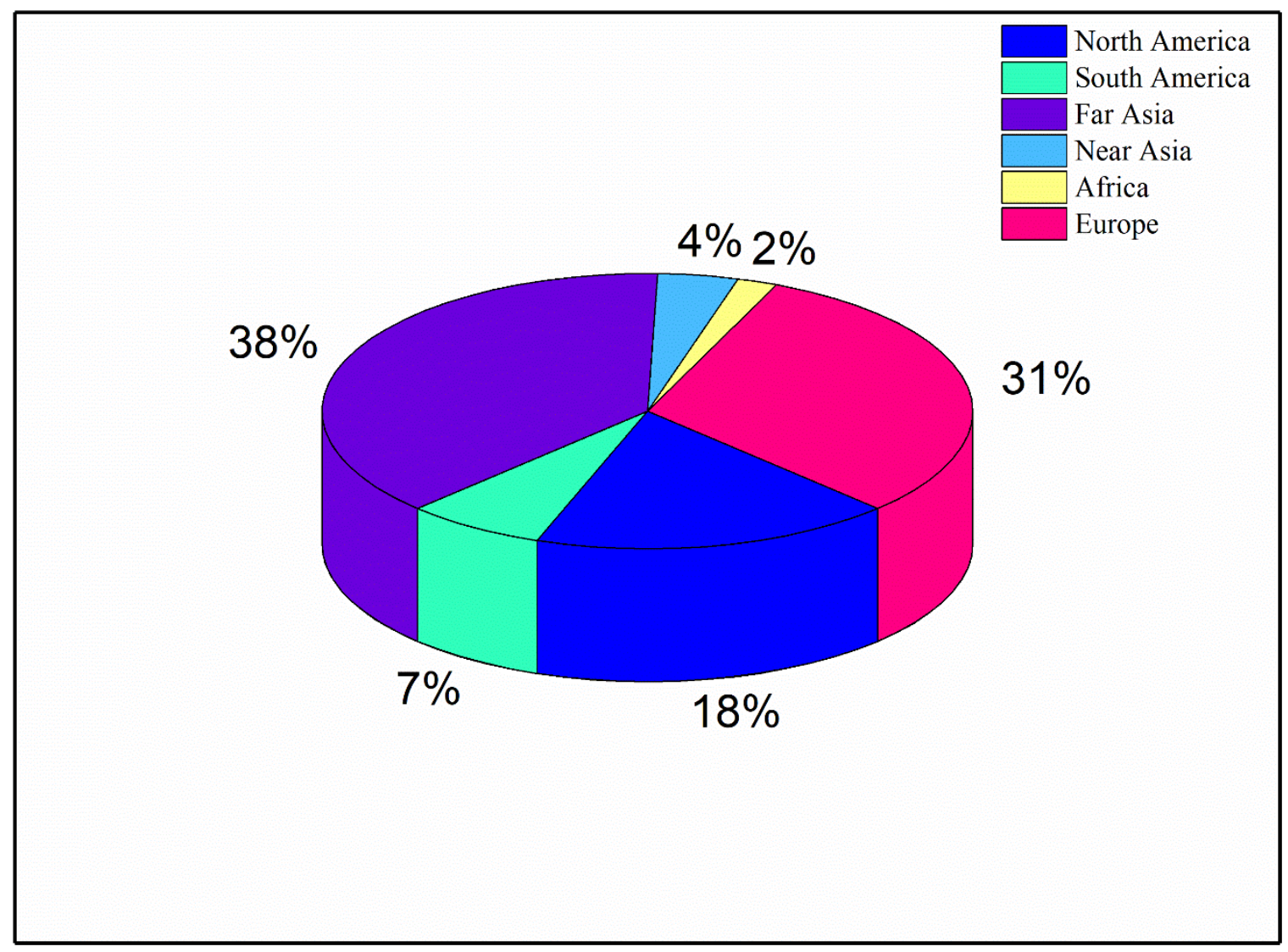

Figure 1.1 Worldwide distribution of $\mathrm{nZnO}$ usage (data adapted from Kołodziejczak-Radzimska (2014). 


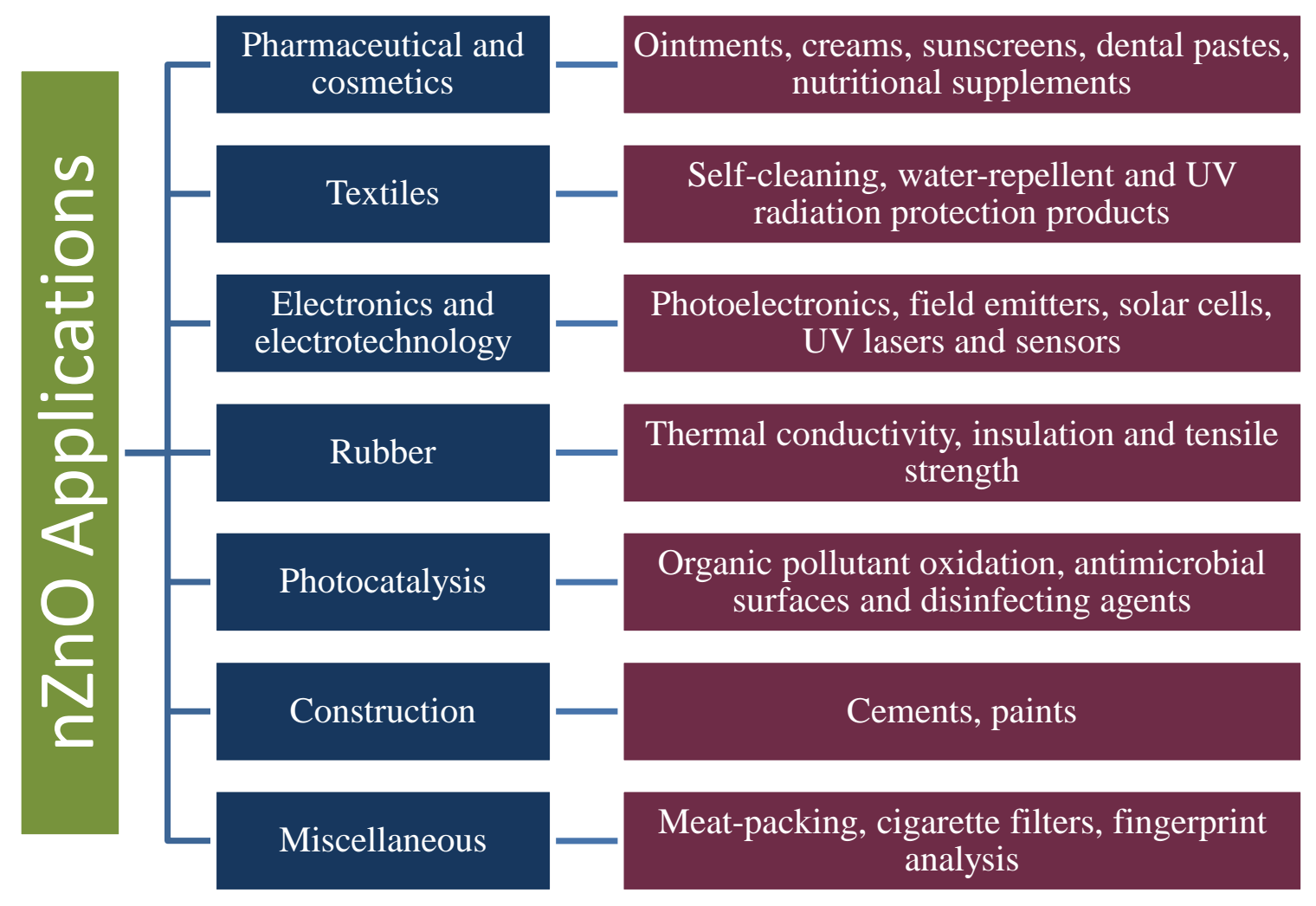

Figure 1.2 Schematic representation of the application of $\mathrm{nZnO}$ 
Table 1.1 Common liquid and gas phase chemical synthesis methods for $\mathrm{nZnO}$ including potential applications found in literature.

\begin{tabular}{|c|c|c|c|}
\hline Classification & Synthesis Category & Applications & Reference \\
\hline Physical phase & Mechanochemical process & Photocatalyst and heterogenous catalyst & [14-18] \\
\hline \multirow{5}{*}{ Liquid phase } & Precipitation process & Photocatalysis, dielectrics and antimicrobial activity & {$[19-21]$} \\
\hline & Sol-gel & Sarin degradation, cellular imaging and water treatment & {$[22-27]$} \\
\hline & $\begin{array}{l}\text { Solvothermal, } \\
\text { hydrothermal and } \\
\text { microwave techniques }\end{array}$ & Electronics, photocatalysis and gas sensing & {$[31-34]$} \\
\hline & Microemulsions & Voltage surge devices, photocatalysis and gas sensing & [35-37] \\
\hline & Green synthesis & Antimicrobial and antifungal treatments & [41] \\
\hline \multirow{2}{*}{ Gas Phase } & Inert gas condensation & Electrical, optical and magnetic application & [46] \\
\hline & Flame spray pyrolysis & Aerosol treatment for in vivo studies and catalysts & {$[49,52-54]$} \\
\hline
\end{tabular}




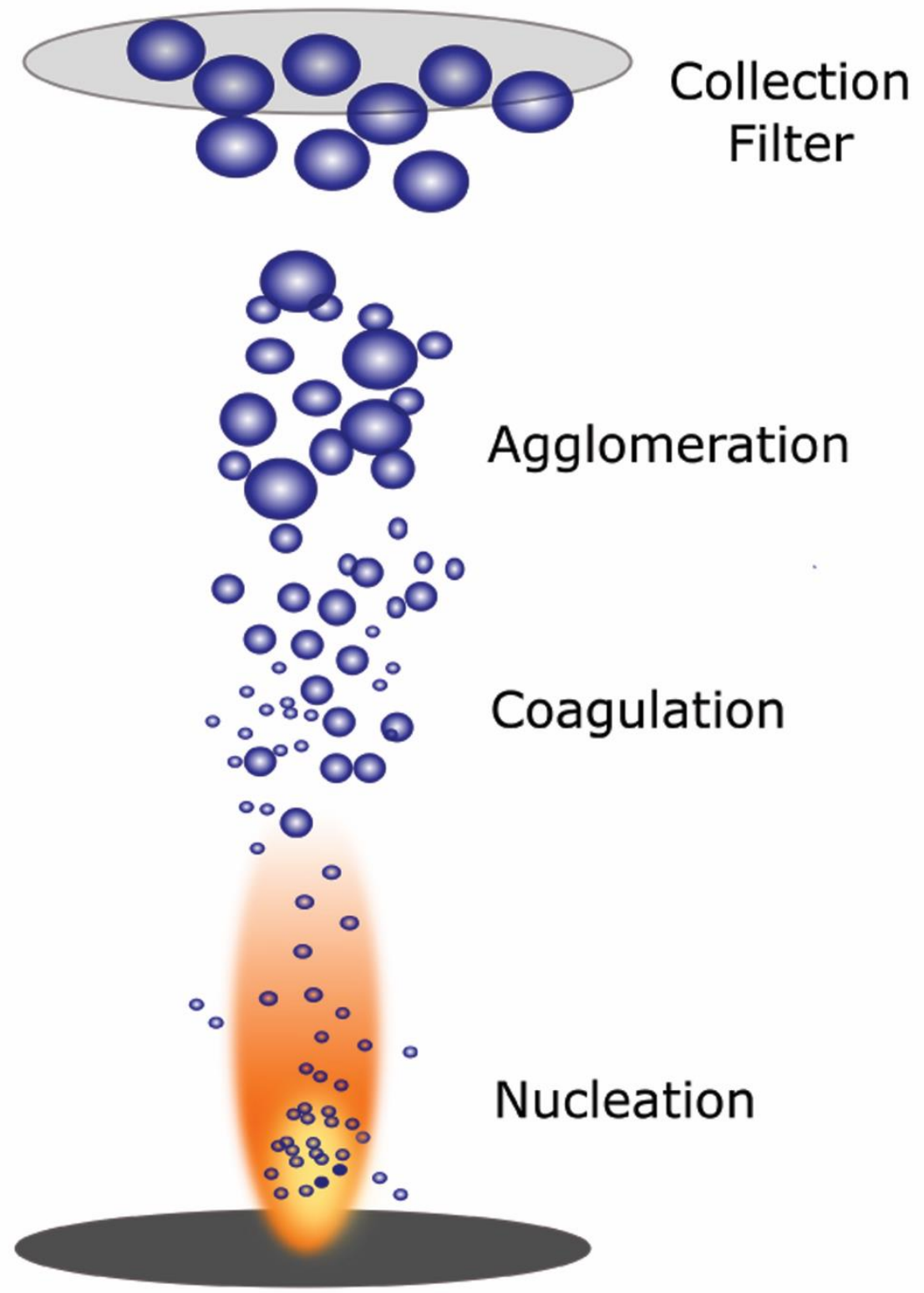

Figure 1.3 Reaction steps in the formation of $\mathrm{nZnO}$ during flame spray pyrolysis. 


\section{Exited State}

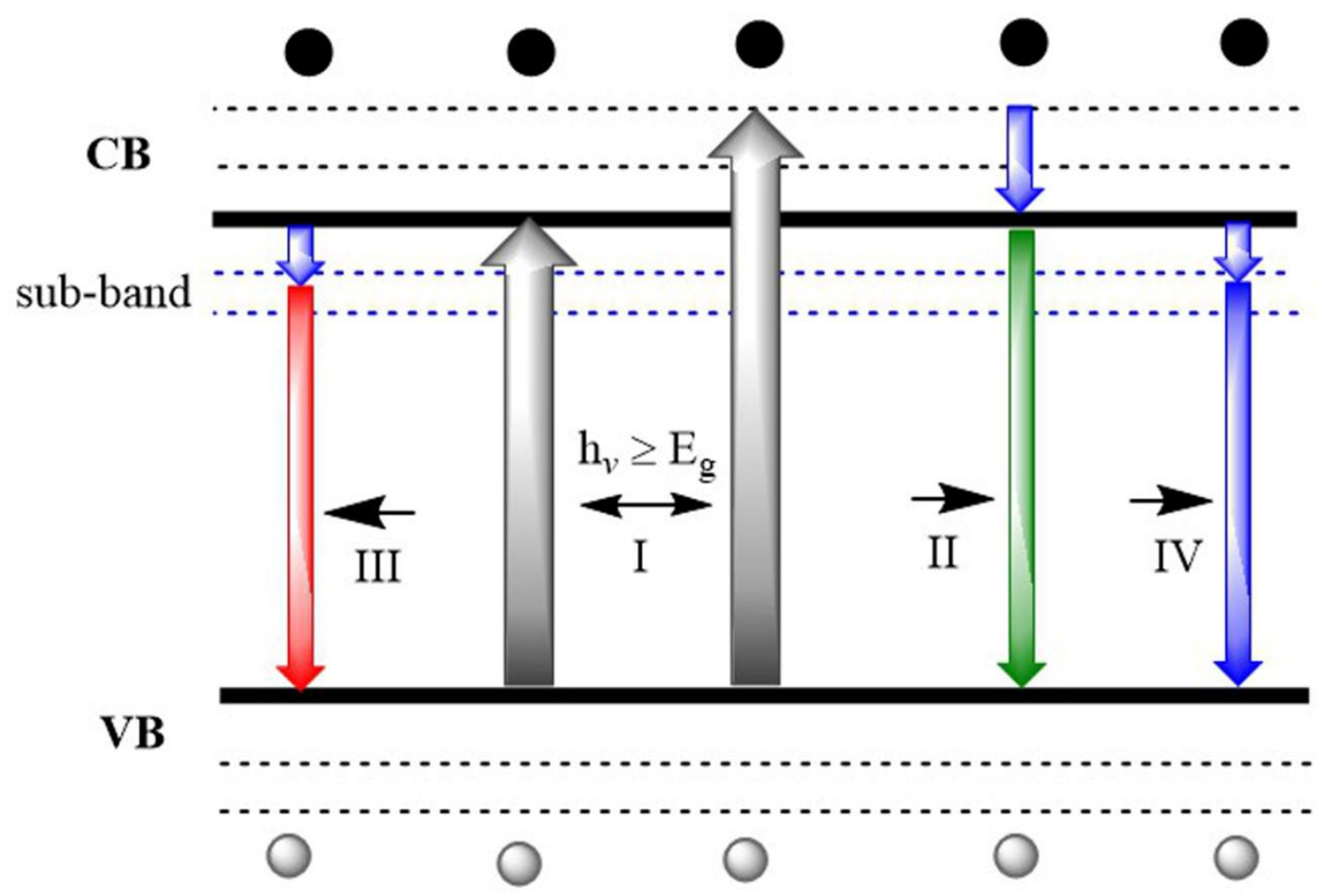

\section{Ground State}

Figure 1.4 The main photophysical processes of $\mathrm{nZnO}$ when excited by UV radiation where $h_{v} \geq E_{g}$. The four processes represent are: I) photo-excited process (gray arrows), II) band -band photoluminescence process (green arrow), III) excitonic process (red arrow), and IV) non-radiative transition process (blue arrows). The empty spheres in the valance band (VB) represent valance band holes $\left(h_{v b}^{+}\right)$and solid spheres in the conduction band $(\mathrm{CB})$ denote conduction band electrons $\left(\mathrm{e}_{\mathrm{cb}}^{-}\right)$. 


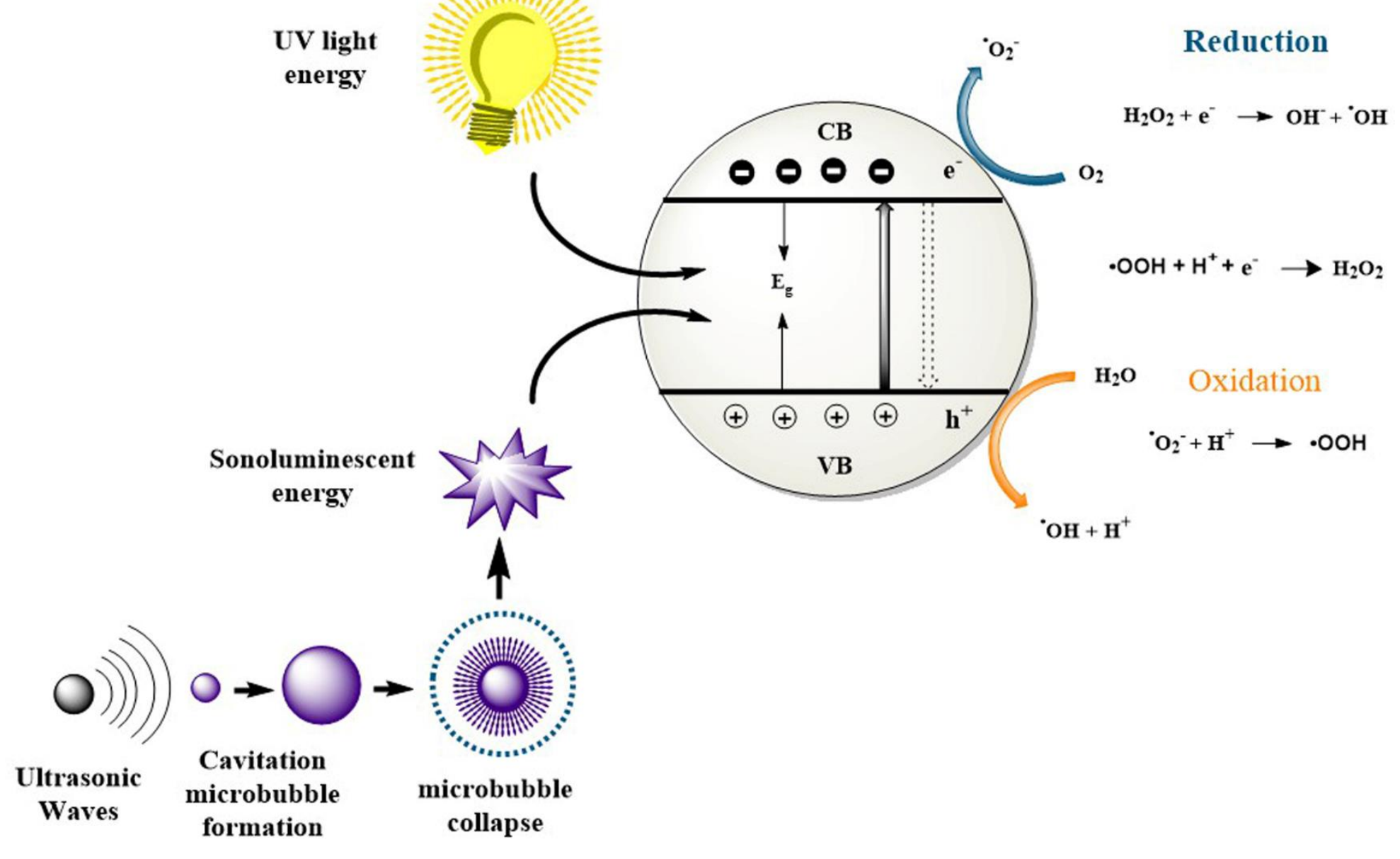

Figure 1.5 Sonocatalytic and photocatalysis reaction processes for $\mathrm{nZnO}$ including redox reactions, reactive intermediates, and reactive oxygen species. 


\section{DLVO Theory}

NP W造数 Proteins

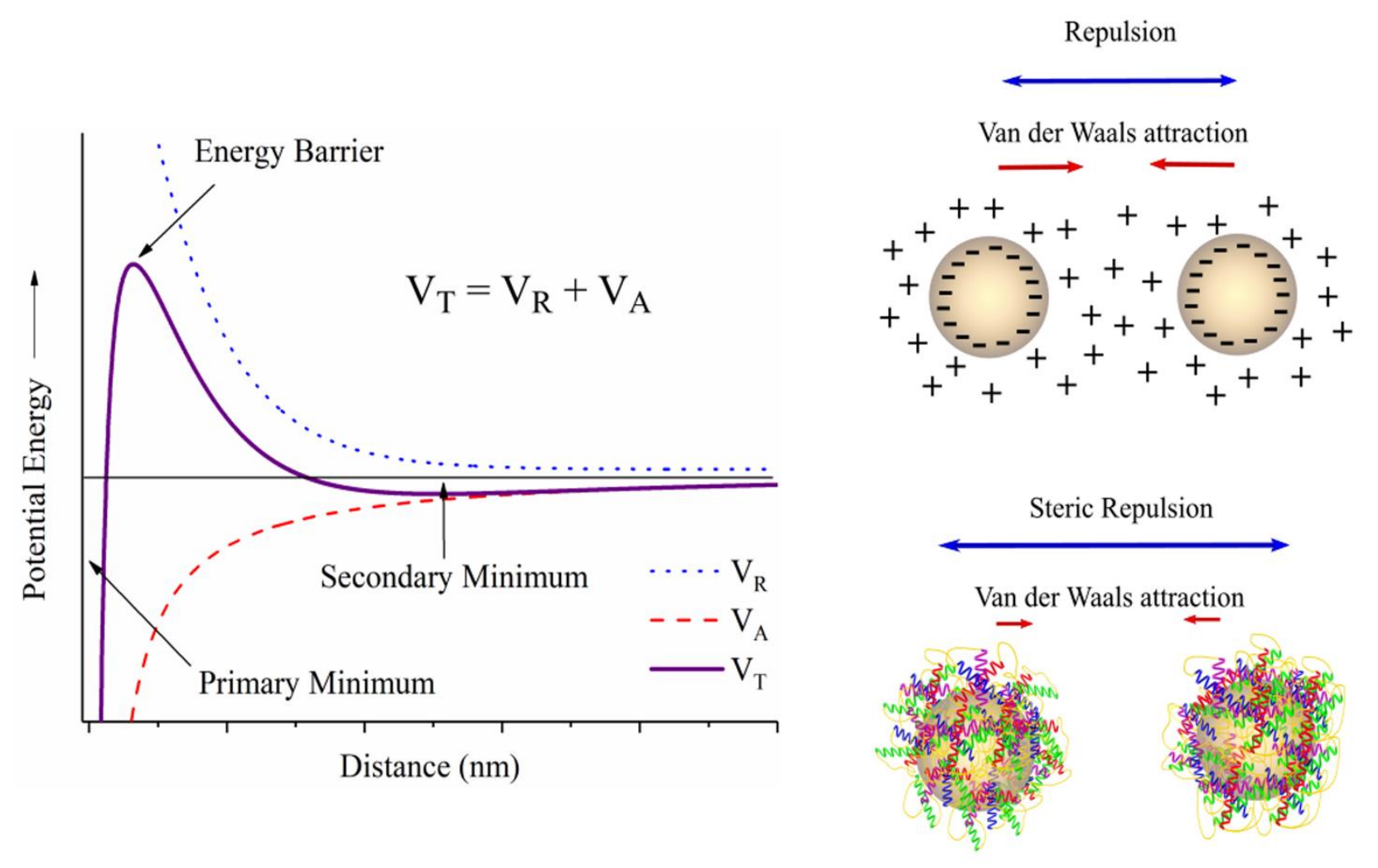

Figure 1.6 DLVO graph depicting colloidal stability for a NP dispersion. 


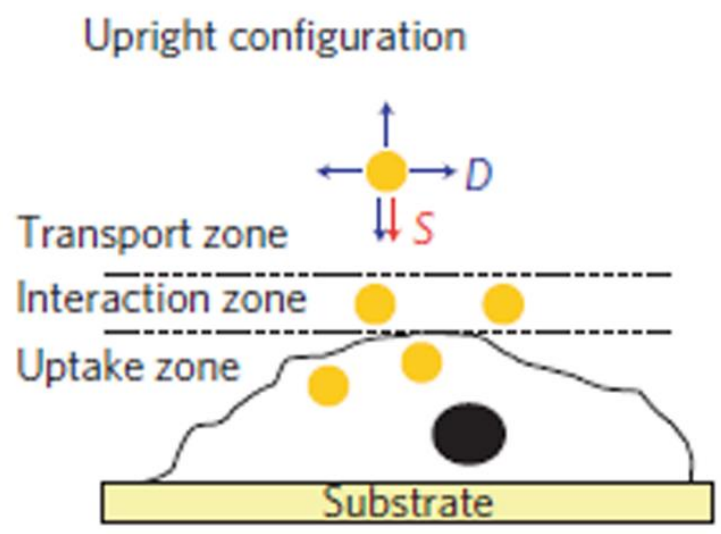

Inverted configuration

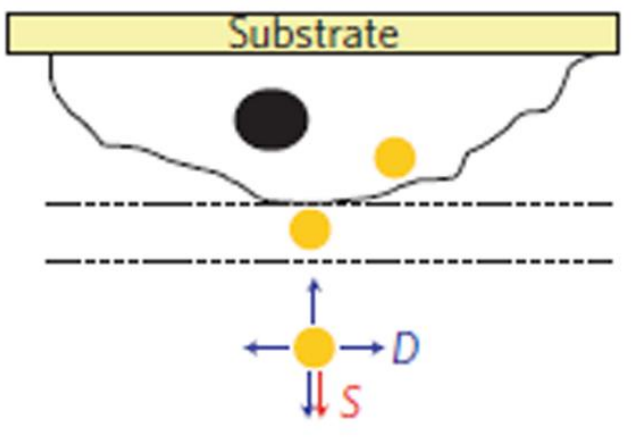

Figure 1.7 Schematic illustrating the transport dynamics of nanoparticles when introduced to adherent cell culture models in both the upright (left side) and inverted (right side) configurations. Cho article Figure 4. Reprinted by permission from Macmillan Publishers Ltd: Nature Nanotechnology (Cho, E. C.; Zhang, Q.; Xia, Y. The effect of sedimentation and diffusion on cellular uptake of gold nanoparticles. Nature Nanotechnology 2011, 6, 385-391.), copyright 2011. 
Adherent Cells

Suspension Cells

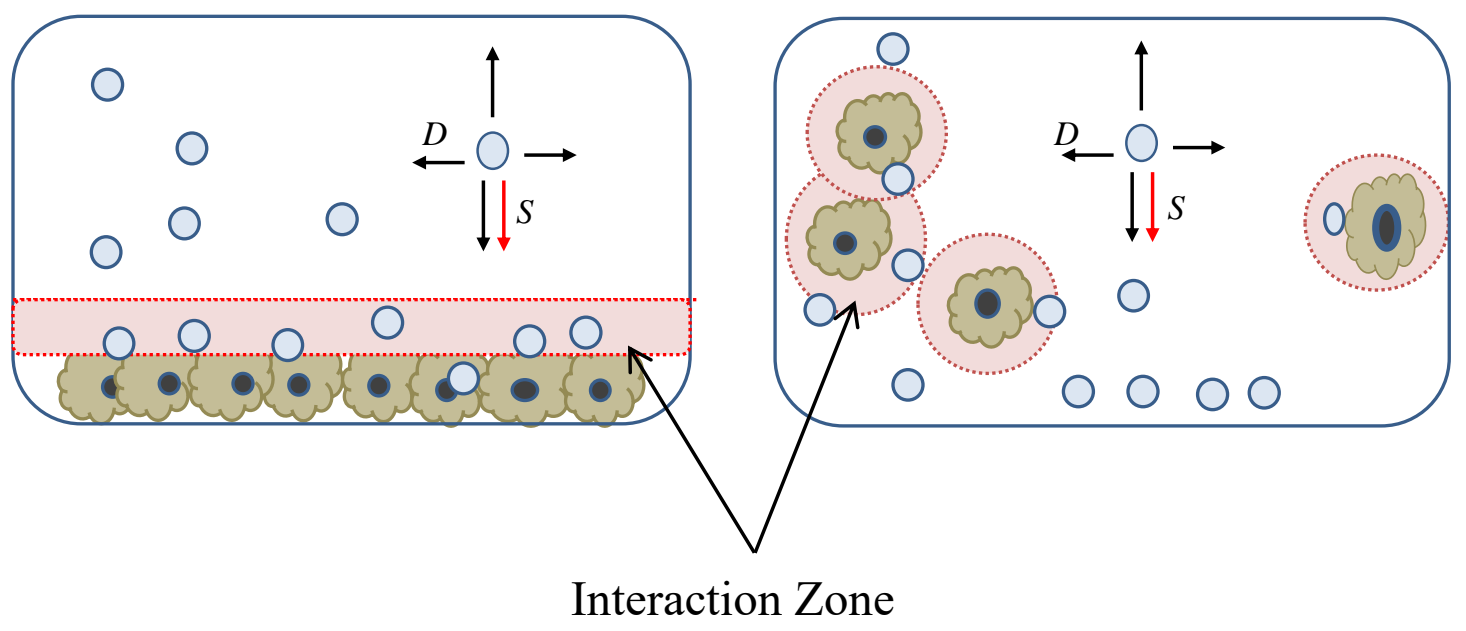

Figure 1.8 Schematic illustrating dispersion dynamics within cell culture. 


\section{nZnO Dissolution in Cellular Media}

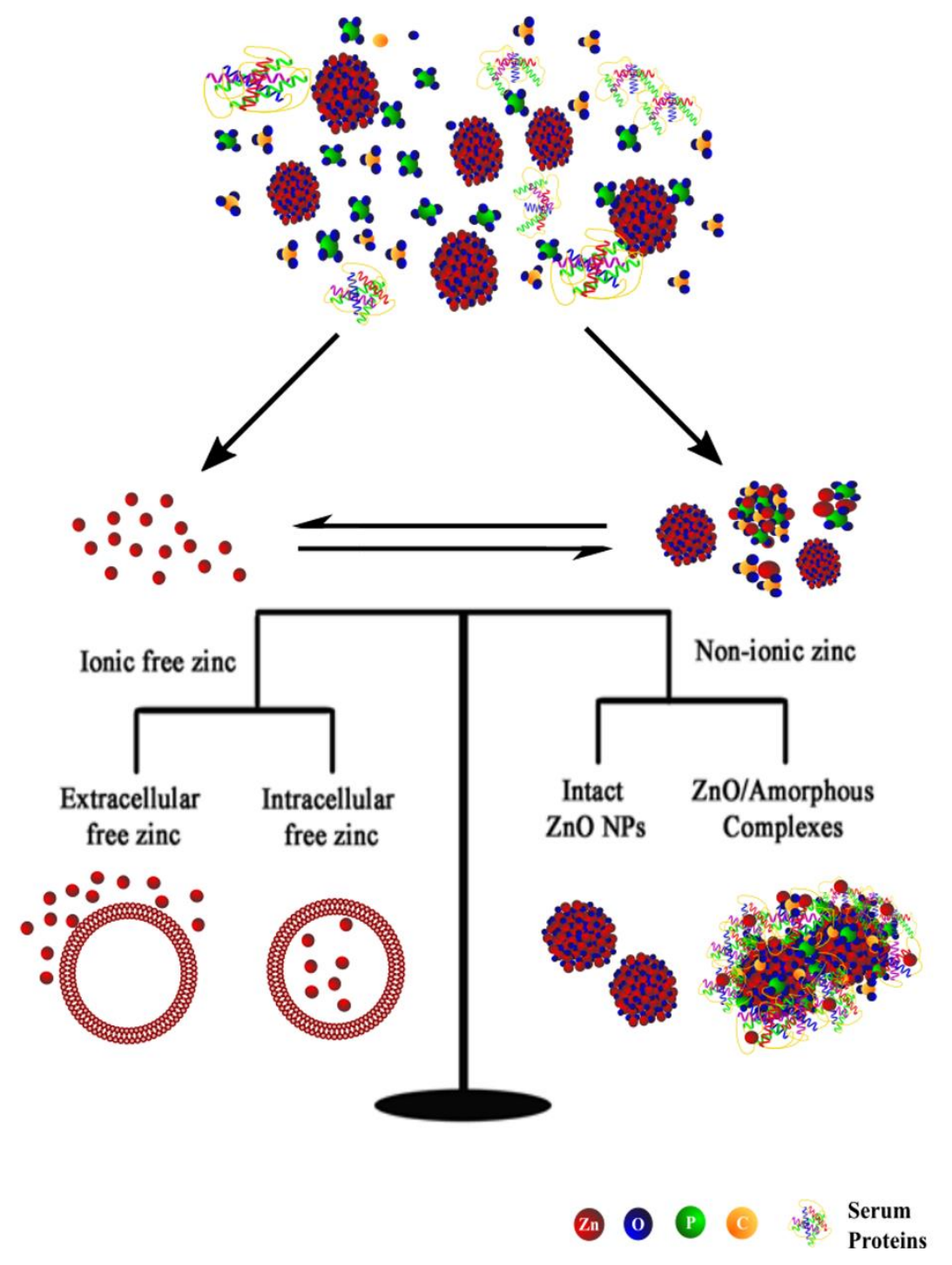

Figure 1.9 Schematic depicting the dissolution processes associated with nZno. 

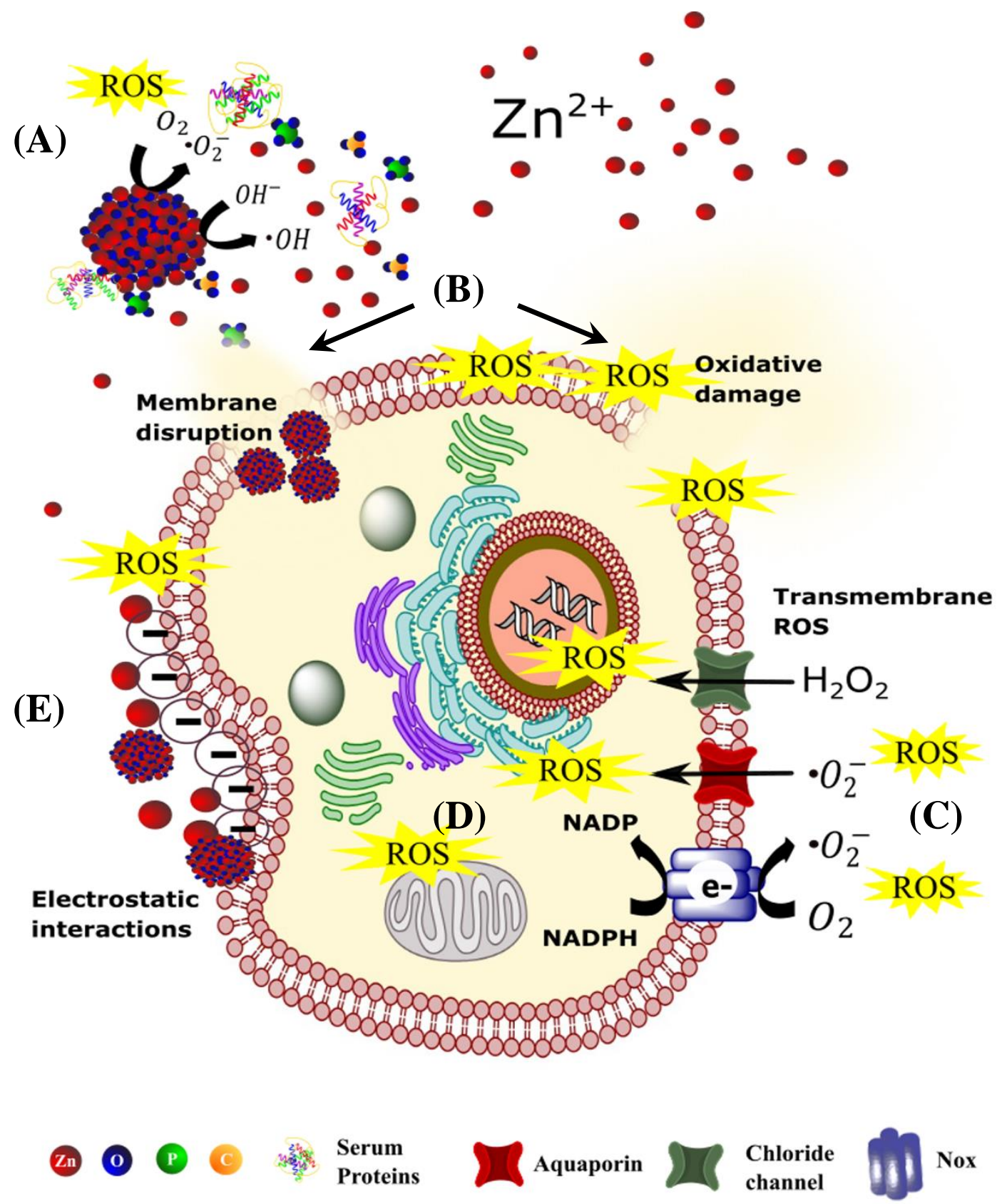

Figure 1.10 Schematic illustrating the various mechanisms by which (A) exogenous ROS generated at the $\mathrm{nZnO}$ surface and the $\mathrm{nZnO}$ particles themselves can induce cellular damage including (B) membrane disruption and lipid peroxidation. (C) Internalized ROS via membrane bound transporters will cause (D) oxidative damage to cellular components or activate stress-induced signaling. (E) Electrostatic interactions of NPs with cellular membranes will facilitate NP adsorption and cellular membrane damage. 

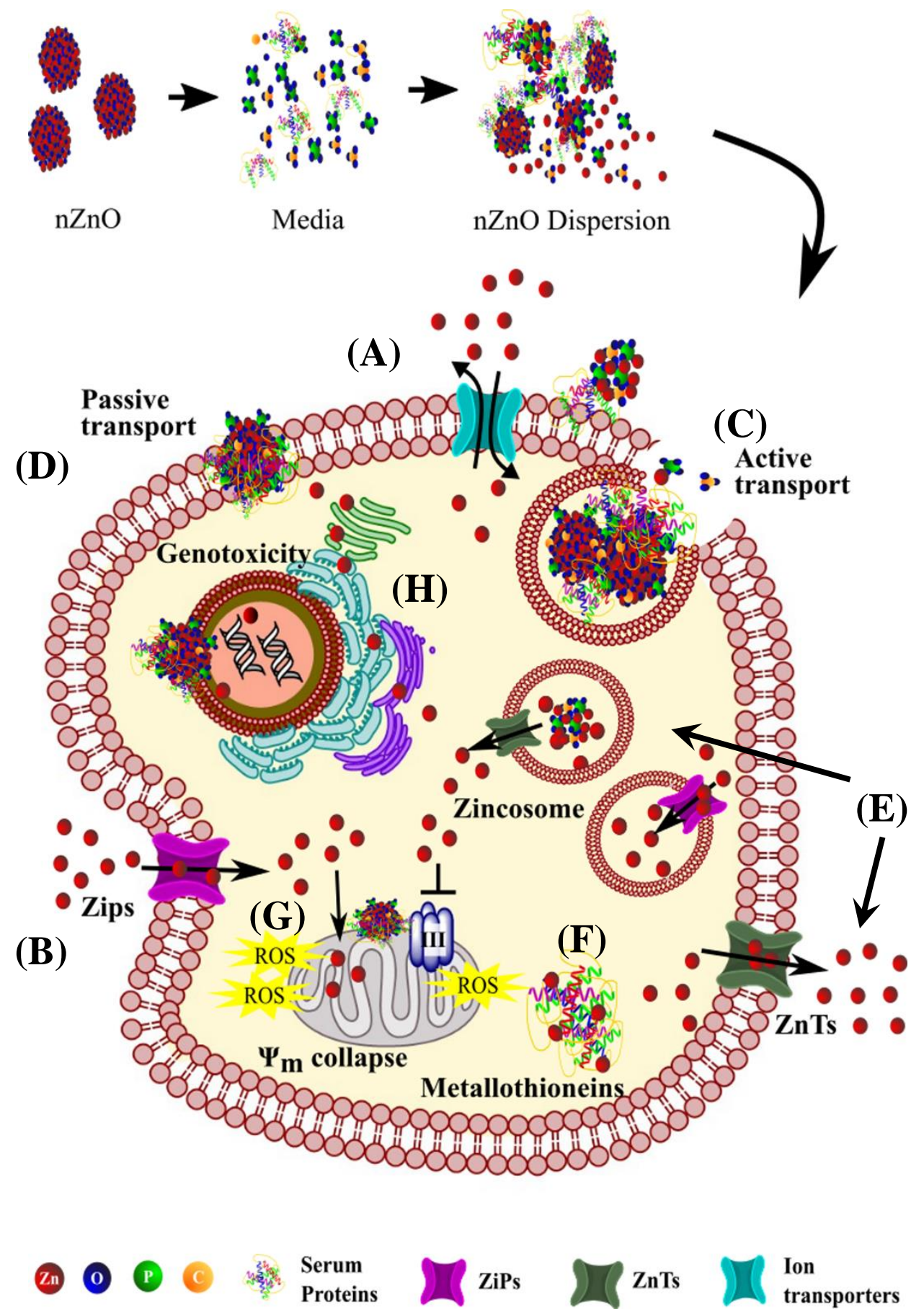

Figure 1.11 Schematic illustrating the various mechanisms by which extracellular, intracellular free, and cell-associated zinc induce cellular damage. Extracellular free zinc can enter cells through (A) non-specific ion channels, and (B) specialized zinc importers (Zips). Intact NPs or amorphous complexes may be internalized through (C) active or (D) passive transport mechanisms. Elevated intracellular free zinc concentrations are remediated through sequestration into $(E)$ zincosomes or by $(F)$ metallothioneins. Disruption of zinc homeostasis can result in (G) mitochondrial and organelle damage or $(\mathrm{H})$ genotoxicity. 


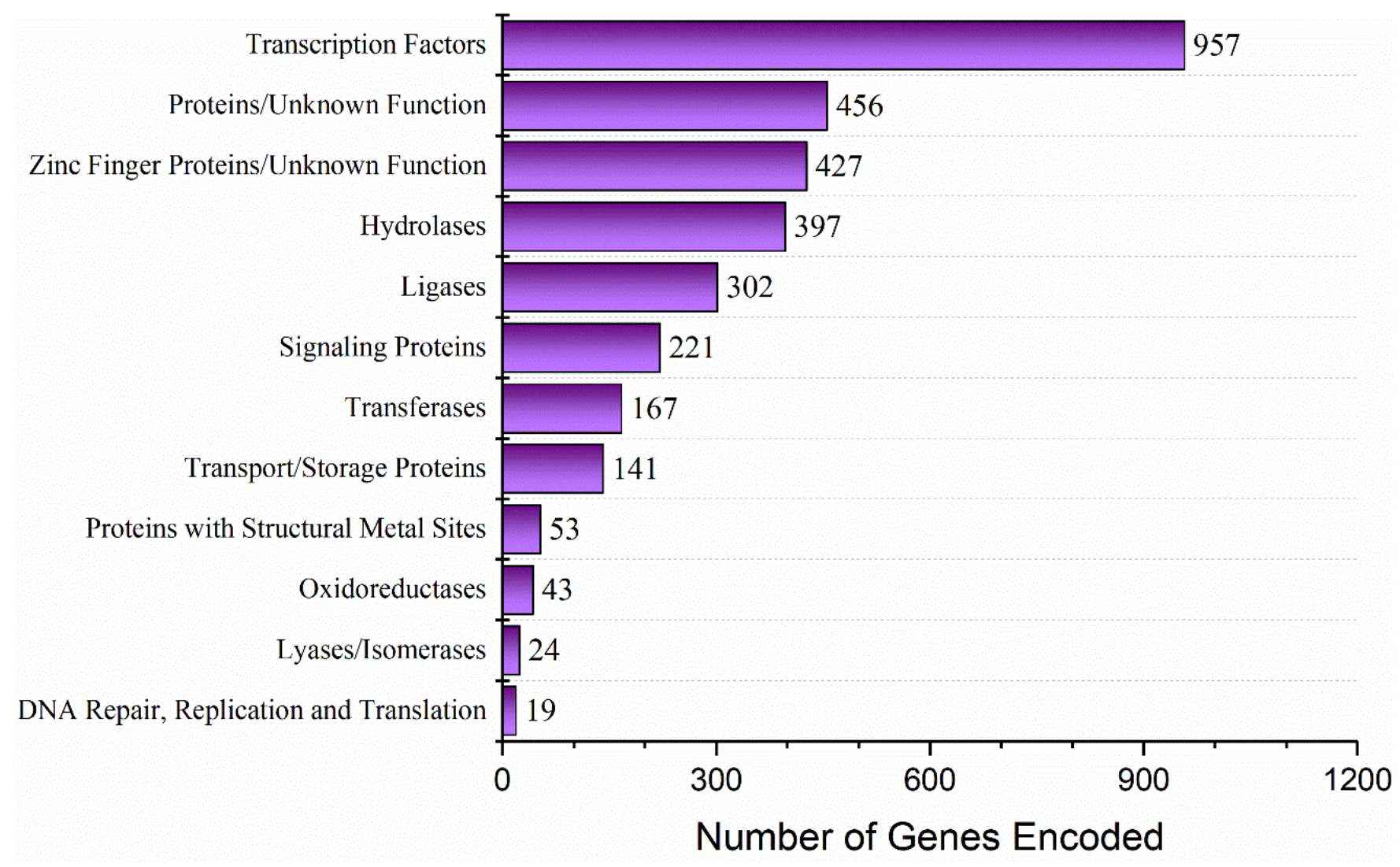

Figure 1.12 The number of human genes encoding for proteins with a zinc-binding site. 


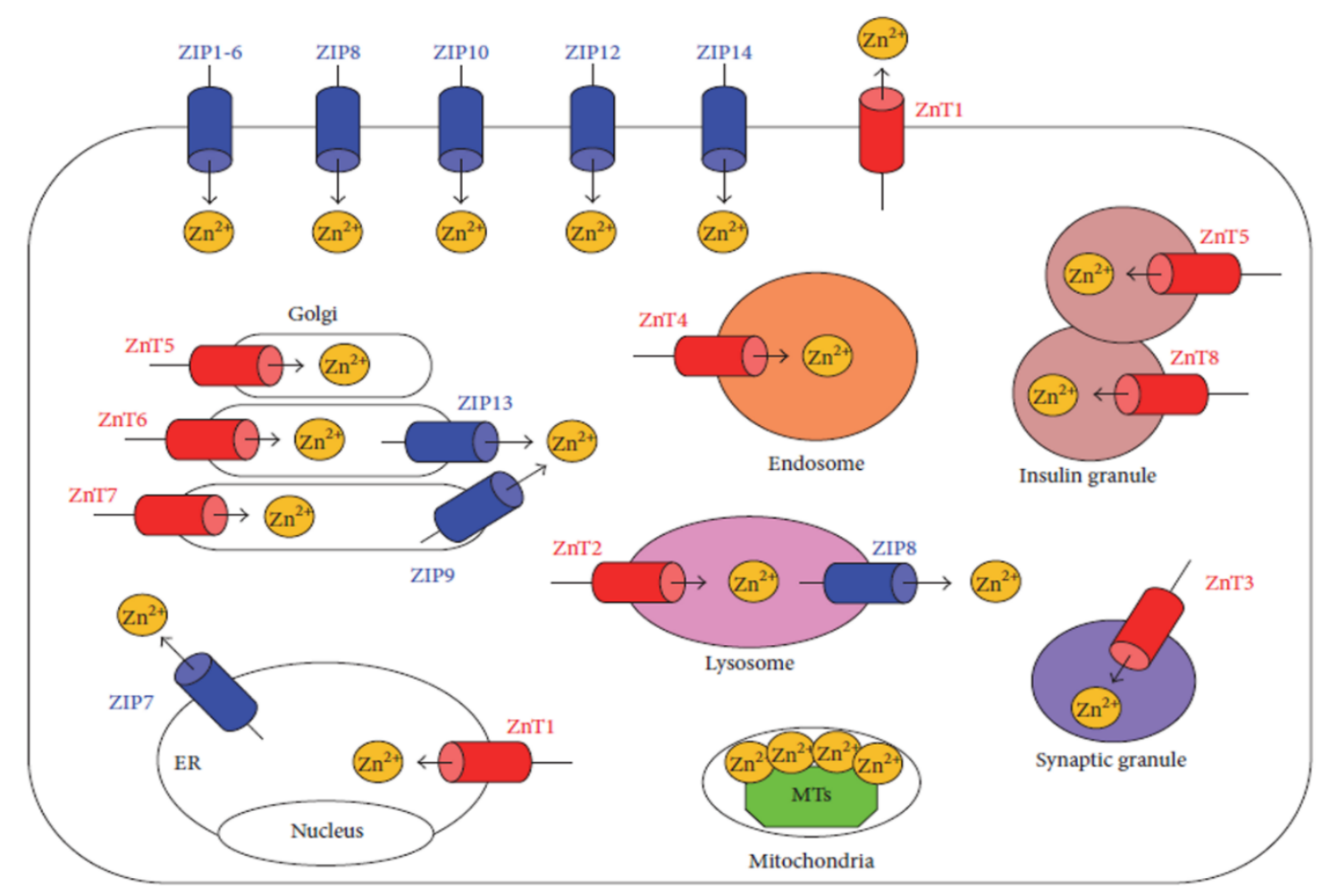

Figure 1.13 Schematic illustrating the cellular localization of the $S L C 39$ (Zips) and SLC30 (ZnTs) families of zinc transporters. Zip proteins (blue) elevate cytosolic zinc levels by importing ionic zinc from the extracellular environment or cellular compartments. ZnT transporters lower cytosolic zinc through efflux into cellular compartments or out of the cell. Metallothioneins contribute to intracellular zinc level management by sequestering zinc ions. Hojya and Fukada article Figure 2. Reprinted by permission from Hindawi Publishers: Journal of Immunology Research (Shintaro Hojyo and Toshiyuki Fukada, "Roles of Zinc Signaling in the Immune System," Journal of Immunology Research, vol. 2016, Article ID 6762343, 21 pages, 2016. doi:10.1155/2016/6762343), copyright 2016. 
Early Zinc Signaling

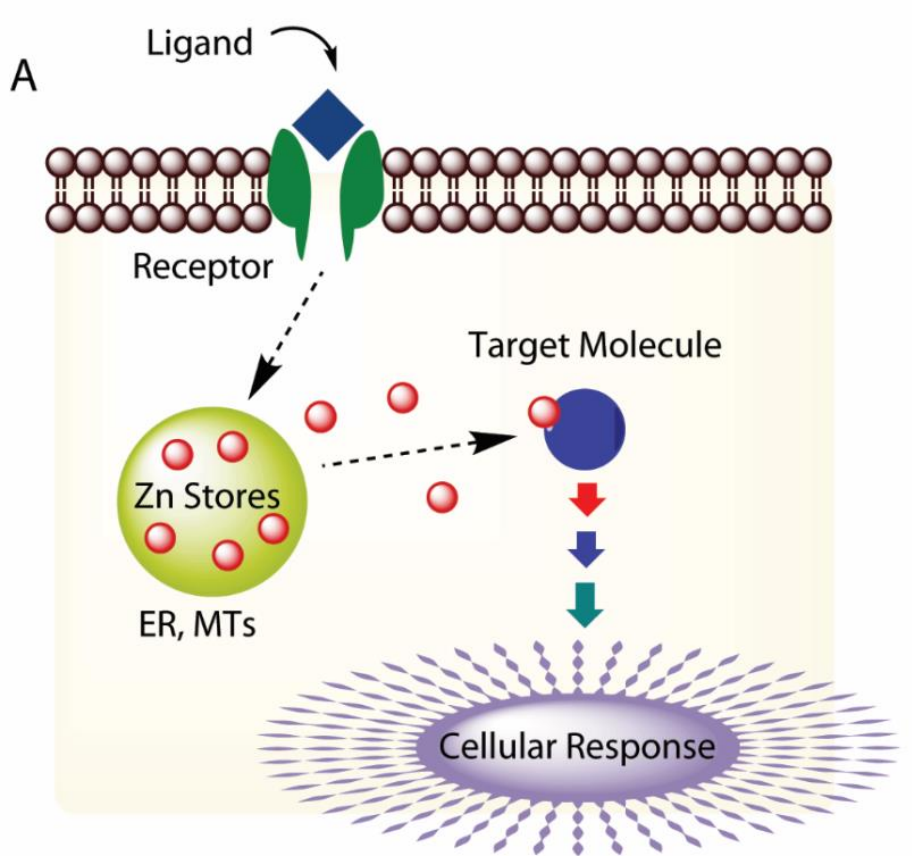

Transcription Independent
Late Zinc Signaling

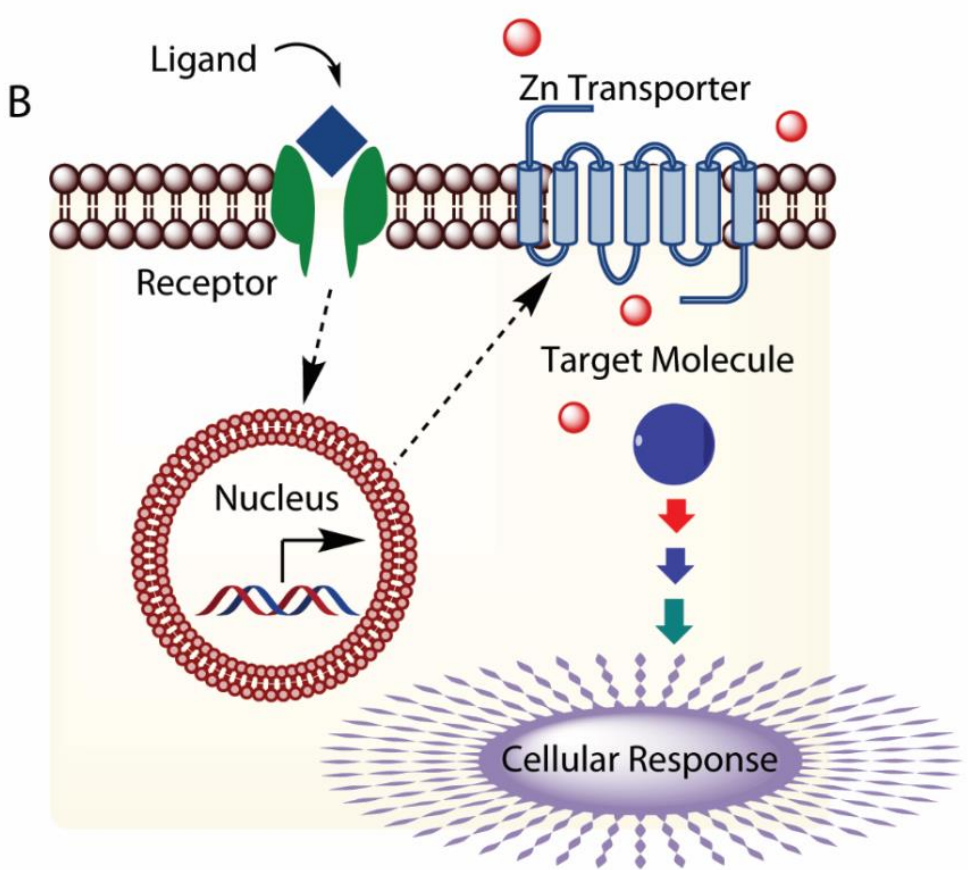

Transcription Dependent

$\mathrm{t}=$ minutes

$t=$ hours

Figure 1.14 Early (A) and late (B) zinc signaling. 


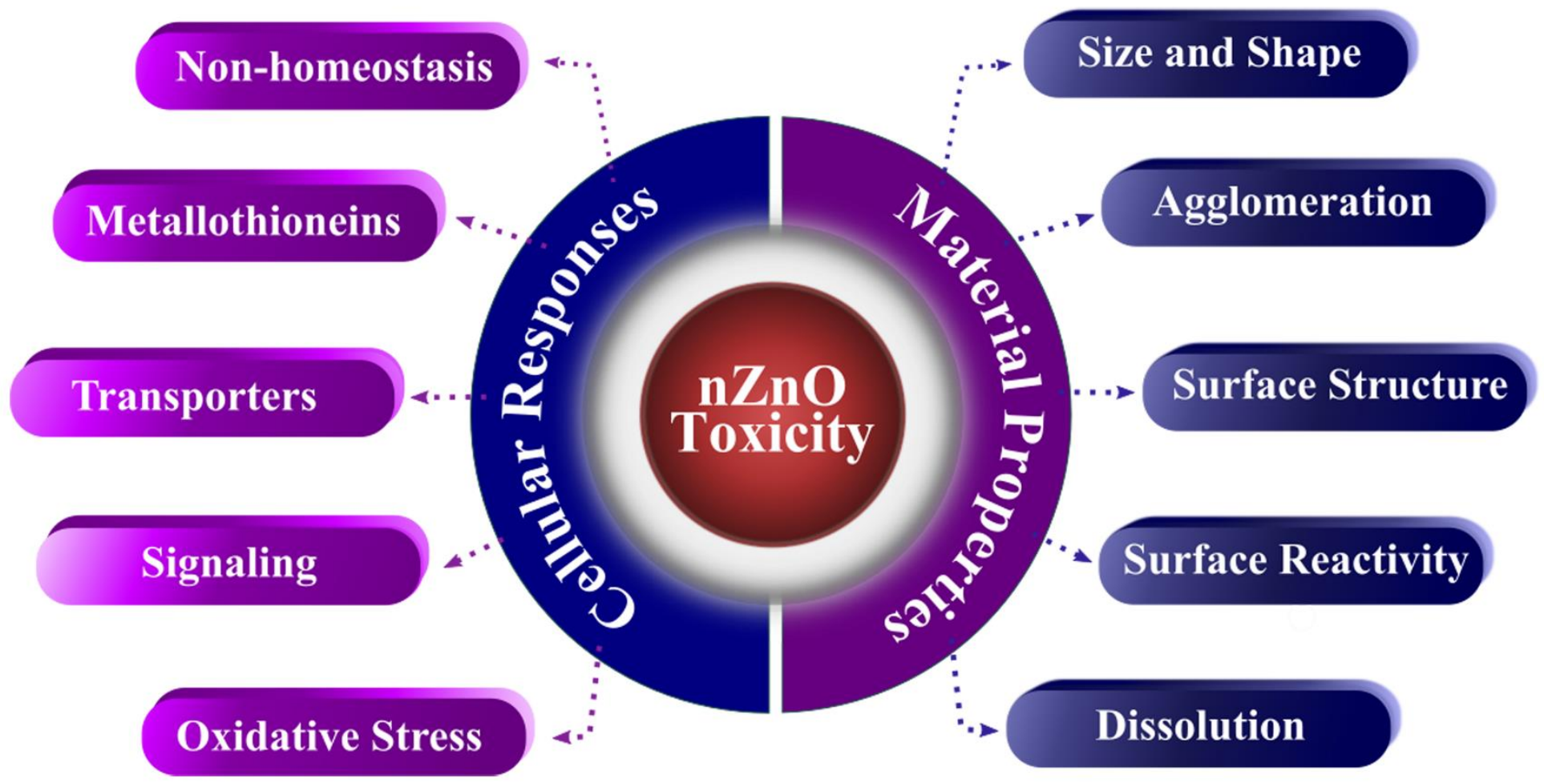

Figure 1.15 Schematic overview summarizing the toxic effect of $\mathrm{nZnO}$. The key material factors implicated in NP- induced toxicity include particle size and shape, agglomeration potential, surface structure and reactivity, and NP dissolution. The observed cellular responses include disruption of zinc homeostasis, metallothionein and zinc transport regulation, zinc related cell signaling, and oxidative stress. 


\section{CHAPTER 2 SERUM PROTEINS ENHANCE DISPERSION STABILITY}

\section{AND INFLUENCE THE CYTOTOXICITY AND DOSIMETRY OF ZNO \\ NANOPARTICLES IN SUSPENSION AND ADHERENT CANCER CELL}

\section{MODELS}

Catherine B. Anders ${ }^{1,2}$, Jordan J. Chess ${ }^{1,4}$, Denise G. Wingett ${ }^{2,3}$ and Alex Punnoose ${ }^{1,2 *}$

${ }^{1}$ Department of Physics, Boise State University, Boise, ID 83725

${ }^{2}$ Biomolecular Sciences PhD program, Boise State University, Boise, ID 83725

${ }^{3}$ Department of Biological Sciences, Boise State University, Boise, ID 83725

${ }^{4}$ Department of Physics, University of Oregon, Eugen, OR 97403

*Corresponding author, e-mail: apunnoos@boisestate.edu

Citation: Anders, C.B., Chess, J.J., Wingett, D.G. and Punnoose, A. Nanoscale Res Lett (2015) 10: 448. https://doi.org/10.1186/s11671-015-1158-y

DOI $\quad$ https://doi.org/10.1186/s11671-015-1158-y

Publisher Springer US

Print ISSN 1931-7573

Online ISSN $1556-276 \mathrm{X}$

Changes No significant changes were made to this publication. 


\begin{abstract}
Agglomeration and sedimentation of nanoparticles (NPs) within biological solutions is a major limitation in their use in many downstream applications. It has been proposed that serum proteins associate with the NP surface to form a protein corona that limits agglomeration and sedimentation. Here, we investigate the effect of fetal bovine serum (FBS) proteins on the dispersion stability, dosimetry and NP-induced cytotoxicity of cationic zinc oxide nanoparticles $(\mathrm{nZnO})$ synthesized via forced hydrolysis with a core size of $10 \mathrm{~nm}$. Two different in vitro cell culture models, suspension and adherent, were evaluated by comparing a (phosphate buffered saline PBS) nZnO dispersion (nZnO/PBS) and an FBS-stabilized PBS nZnO dispersion (nZnO - FBS/PBS). Surface interactions of FBS on $\mathrm{nZnO}$ were analyzed via spectroscopic and optical techniques. FTIR confirmed the adsorption of negatively charged protein components on the cationic $\mathrm{nZnO}$ surface through the disappearance of surfaced-adsorbed carboxyl functional groups and the subsequent detection of vibrational modes associated with the protein backbone of FBS-associated proteins. Further confirmation of these interactions was noted in the isoelectric point shift of the $\mathrm{nZnO}$ from the characteristic $\mathrm{pH}$ of 9.5 to a $\mathrm{pH}$ of 6.1 . In $\mathrm{nZnO}-\mathrm{FBS} / \mathrm{PBS}$ dispersions, the FBS reduced agglomeration and sedimentation behaviors to impart long term improvements (>24 hours) to the $\mathrm{nZnO}$ dispersion stability. Furthermore, mathematical dosimetry models indicate that $\mathrm{nZnO}-\mathrm{FBS} / \mathrm{PBS}$ dispersions had consistent NP deposition patterns over time unlike unstable $\mathrm{nZnO} / \mathrm{PBS}$ dispersions. In suspension cell models, the stable $\mathrm{nZnO}-\mathrm{FBS} / \mathrm{PBS}$ dispersion resulted in a $\sim 33 \%$ increase in the NPinduced cytotoxicity for both Jurkat leukemic and Hut-78 lymphoma cancer cells. In contrast, the $\mathrm{nZnO}-\mathrm{FBS} / \mathrm{PBS}$ dispersion resulted in $49 \%$ and $71 \%$ reductions in the
\end{abstract}


cytotoxicity observed towards the adherent breast (T-47D) and prostate (LNCaP) cancer cells lines, respectively. Presence of FBS in the NP dispersions also increased the reactive oxygen species generation. These observations indicate that the improved dispersion stability leads to increased NP bioavailability for suspension cell models and reduced NP sedimentation onto adherent cell layers resulting in more accurate in vitro toxicity assessments.

\section{Keywords}

Zinc oxide nanoparticles, dispersion stability, nanotoxicity, dosimetry

\section{Background}

When prepared in nanostructured form, many materials develop novel and unique physico-chemical properties leading to their use in nanotechnology applications such as quantum dots and MRI contrast agents for imaging [1], biosensors that use surface plasmon resonance [2], and nanomedicinal approaches that employ hyperthermia and photothermal therapy [3]. Efficient use of NPs and nanocarriers in biomedical applications, such as delivery of therapeutic and imaging agents into tumor sites and genetic materials into cells, has been successfully demonstrated in recent years [4-6]. Interestingly, cytotoxicity is a property that is inherent in many of these commonly used materials when they are synthesized with their crystallite size in the nanoscale range [7-9]. This has raised significant scientific and public concerns on the environmental and health effects of every nanomaterial before employing them in nanotechnology applications. It is therefore necessary to evaluate the cytotoxic response of nanomaterials and the underlying mechanisms of nanomaterial toxicity to accurately address the growing concerns about the impact of the unwanted cytotoxicity of nanomaterials. 
Numerous studies reported in the literature have shown that NP-induced toxicity depends on the nanomaterial properties such as crystallite size [10, 11], shape [12], charge [13], and chemical phase [14]. Some studies have also shown that the toxic response of a given nanomaterial might vary with the specific cell/organism type such as prokaryotes vs. eukaryotes [15], gram positive bacteria versus gram negative bacteria, and normal cells versus cancerous cells [16]. These observations suggest that the role of organism-specific factors such as surface structure of cells $[17,18]$ and the proliferation status $[10,16]$ also influence the toxic response. Although the cytotoxicity of nanomaterials is an unfavorable feature for many applications, it could become very useful for certain therapeutic applications, if the toxicity is cell- or organism-specific. The cytotoxicity reported for most nanomaterials does not display a cell-specific or organism-specific behavior. However, many groups $[16,19,20]$, including the authors, have demonstrated that certain $\mathrm{ZnO} \mathrm{NP}$ formulations show a strong preferential cytotoxicity to cancer cells of identical lineage. For example, while resting primary human immune cells ( $\mathrm{T}$ lymphocytes) showed no appreciable effect when treated with $\leq 5 \mathrm{mM}$ concentrations of $\mathrm{ZnO}$ NPs, cancer cells of the same lineage showed significantly increased NP-induced toxicity resulting in approximately 28-35-fold increases in NP sensitivity $[10,16]$. Thus, toxicity studies are extremely important both to assess the environmental and health impacts of NPs and to modify the nanomaterial design to make them safer, as well as to develop novel nanomedicinal approaches utilizing cell-specific cytotoxicity.

Considering the large number and types of new nanomaterials being developed and coming to the market, in vitro studies are essential in assessing the toxicity in highthroughput systems as they provide rapid and cost-effective screening. In most in vitro 
cytotoxicity experiments, cells are cultured in a culture medium to which a prepared dispersion of the NPs in a suitable solution is introduced. In contrast, in vivo toxicology studies frequently employ NPs dispersed in a suitable medium that are subsequently injected into the blood vessels or tissues of the animal model. If successful, the dispersed NPs will reach tumor sites and achieve deep penetration and entrapment in the cancerous tissue by means of the enhanced permeability and retention (EPR) effect, characterized by increased vascular permeability and compressed lymphatic networks in solid tumors [21]. However, making highly stable and efficient dispersions of nanoparticles to achieve efficient nanoparticle delivery for both in vitro and in vivo applications has been a challenging task. A major difference that NPs possess compared to the well-studied conventional toxic chemicals (which are well soluble molecules and ions) is the difficulty in dispersing them in suitable solutions and culture media, and to maintain them as stable dispersions for sufficient durations [22]. For most in vitro studies, NP suspensions should remain stable at least for 24 hours while in vivo studies require stability for much longer durations [23]. Formation of large agglomerates and their sedimentation over longer periods often prevents a significant fraction of the NPs from participating in efficient interactions with the test cells and the resulting cytotoxic response.

Nanoparticle dispersion stability is a complex interplay between the agglomerate size of NP complexes in dispersion (hydrodynamic size) and their sedimentation behavior over time [24]. Factors that affect NP stability include NP size [25, 26], surface structure $[18,27]$, capping agents [28], $\mathrm{pH}$ [29], ionic strength [29], organic and protein components $[26,27,30]$, and free ions in the solution system [31]. Although it is now known that the physicochemical properties of the nanomaterial, the specific cell/organism type and the 
membrane properties $[10,17,18]$ of the test organism play a crucial role in the resulting cytotoxic response, very few studies have focused on the important role of NP dispersion characteristics and dispersion stability and the effects of NP agglomeration and sedimentation in the resulting toxicity [32, 33]. Furthermore, Cho et al. [34] have recently shown that the cellular uptake of gold NP in breast cancer cells measured using conventional upright in vitro cell culture configuration was significantly higher than that obtained when an inverted set-up was used, suggesting the effect of NP sedimentation and diffusion in the media play a significant role in the NP-cell interaction. In addition, suspension stability has been shown to directly influence effective administered dose of NPs to adherent cells grown in vitro [22, 32, 33].

When NPs are introduced to biological fluids such as human plasma, a biomolecular corona consisting of proteins and small molecules rapidly forms on the NP surface [27, 35-37]. Since the blood circulatory system is the most probable treatment administration option for NPs into the human body, it is important to investigate how adsorption of blood proteins on NPs will affect the cellular response, and more importantly, the differential cytotoxicity of $\mathrm{ZnO}$ NPs between cancerous and normal cells. The effect of having serum in culture medium and/or adsorption of the serum proteins on some NP systems such as silica [38], carbon nanotubes [39], graphene oxide [40] and $\mathrm{ZnO}$ [41, 42] have been studied recently. In all these cases, the primary effects of serum addition included improved dispersion stability and a clear reduction of the NP-induced cytotoxicity. Interestingly, the above-mentioned studies were conducted using adherent cell models; therefore, comprehensive studies are needed to determine if similar responses are present for suspension cell models as well. Hypothetically, increases in the NP-induced 
toxicity in suspension cell models would be observed as NP sedimentation decreases, thereby improving the bioavailability of the NPs to the cells.

In this study, we investigated the use of FBS as a $\mathrm{ZnO}$ NP coating agent and the effects of this FBS coating the surface structure of the uncoated NPs, the dispersive stability of the NPs in solution and the effective dosimetry of the NPs in cellular toxicity assays. Since NP dispersion stability and dosimetry is highly dependent upon a balance of NP diffusion and sedimentation velocities within solution [32-34, 43, 44], the use of multiple suspension and adherent cell models was necessary to fully characterize any observations attributable to the addition of the FBS. A complete NP characterization profile was employed to fully interrogate NP-surface changes as a result of the FBS coating. In addition, we examined the agglomeration and sedimentation behavior of the FBS-coated $\mathrm{ZnO}$ NPs (nZnO-FBS) compared to uncoated $\mathrm{ZnO} \mathrm{NP}$ (nZnO) in biologically relevant solutions. Finally, we investigated the effects that FBS coating would have on the solubility of the $\mathrm{nZnO}$, the $\mathrm{NP}$-induced toxicity and $\mathrm{nZnO}$-induced reactive oxygen species production. As a whole, this work provides a comprehensive look at effects of FBS as a dispersion stabilizing agent in nanotoxicology studies.

\section{Methods}

\section{Synthesis and Characterization of $\mathrm{nZnO}$}

The nZnO sample used in this study was produced using the forced hydrolysis of $\mathrm{Zn}$ acetate dehydrate precursor in diethylene glycol (DEG). The solution was held at $150{ }^{\circ} \mathrm{C}$ for 90 minutes. Nanopure water was added to the solution at $80{ }^{\circ} \mathrm{C}$ to obtain the desired crystallite size. This synthesis method allows for excellent control of the crystallite size by carefully regulating the temperature parameters and the hydrolysis ratio of the reaction 
media [10]. Once cooled to room temperature, the $\mathrm{nZnO}$ were separated from solution via centrifuging at 20,000 rpm and subsequent washings with ethanol. A dried FBS-coated $\left(\mathrm{nZnO}-\mathrm{FBS}^{\text {dried }}\right)$ sample was prepared by sonicating the $\mathrm{nZnO}$ with FBS for 10 minutes. The resulting suspension was then centrifuged and the resulting pellet rinsed several times with nanopure water to remove any unbound FBS. The pellet was dried overnight at $60^{\circ} \mathrm{C}$ to create a powdered $\mathrm{nZnO}-\mathrm{FBS}^{\text {dried }}$ form for materials characterization. Both NP samples were thoroughly characterized and investigated in detail using x-ray diffraction (XRD), transmission electron microscopy (TEM), zeta potential measurements, UV-vis spectrophotometry, and Fourier transformed infrared spectroscopy (FTIR). X-ray diffraction $(\mathrm{XRD})$ spectra were recorded at room temperature on a Philips X'Pert X-ray diffractometer with a $\mathrm{Cu} \mathrm{K}_{\alpha}$ source $(\lambda=1.5418 \AA)$ in Bragg-Brentano geometry. The loose powder samples were leveled in the sample holder to ensure a smooth surface and mounted on a fixed horizontal sample plane. Lattice parameters and crystal size were analyzed with Rietveld refinement using Materials Analysis Using Diffraction (MAUD) software, corrected for instrumental broadening [45]. TEM and high-resolution TEM analysis was carried out using an FEI Tecnai and FEI Titan respectively. The operating voltage of the Tecnai microscope was $120 \mathrm{kV}$ and $300 \mathrm{kV}$ on the Titan. Image processing was carried out using the Digital Micrograph software from Gatan (Pleasant, California, USA). Roomtemperature optical spectra in the ultraviolet and visible light wavelength ranges were collected using a CARY 5000 spectrophotometer. Zeta potentials of the powdered samples of $\mathrm{nZnO}$ were measured in nanopure water as a function of $\mathrm{pH}$ with a Malvern Zetasizer NanoZS. The temperature was equilibrated to $25^{\circ} \mathrm{C}$, and the $\mathrm{pH}$ was varied in the 6 to 12 range using $1.0 \mathrm{~N} \mathrm{HCl}$ and $1.0 \mathrm{~N} \mathrm{NaOH}$ prior to collecting the data. At least six data 
collections per run were performed on three separate aliquots of the $\mathrm{ZnO}$ suspension for each sample. The Zetasizer unit was also used to measure the hydrodynamic size of nZnO aggregates dispersed in selected media. Several solution systems were used for NP stock preparation and analyzed during this experiment.

\section{$\underline{\text { NP Stock Preparation }}$}

To achieve the most stable dispersion for each solution, the critical material specific delivered sonication energy $\left(\mathrm{DSE}_{\mathrm{cr}}\right)$ for the $\mathrm{ZnO}$ NPs was predetermined for the calorimetrically calibrated JSP Super-sonic bath sonication device employed for this study. A DSE $\mathrm{cr}_{\mathrm{cr}}$ of $181 \mathrm{~J} / \mathrm{mL}$ was established through established protocols outlined by Taurozzi et al. (2011) [46] and employed in multiple dosimetry studies [33, 47]. To achieve the needed DSE $E_{c r}, \mathrm{ZnO}$ NPs were suspended at concentrations of $25 \mathrm{mM}$ and sonicated in the bath sonicator delivering a power of $1.05 \mathrm{~W}$ for a total 30 minutes. Several biologically relevant solutions were prepared per manufacturer's instructions unless otherwise defined. They include nanopure water, PBS, FBS, RPMI (RPMI 1640 buffered with sodium bicarbonate and HEPES, pH adjusted to 7.3), RPMI-based cellular media (RPMI 1640 media supplemented with 10\% FBS and $2 \mathrm{mM}$ L-glutamine, $1.5 \mathrm{~g} / 1$ sodium bicarbonate, $4.5 \mathrm{~g} / \mathrm{l}$ glucose, $10 \mathrm{mM}$ HEPES, and $1.0 \mathrm{mM}$ sodium pyruvate and penicillin-streptomycin) and DMEM-based cellular media.

In addition to single solution NP dispersions, several NP stock dispersions were prepared with FBS. Based on most of the reported protocols found in the literature and our own preliminary data, $10 \%$ FBS addition was found to provide optimal results and was used to prepare these stock dispersions. The stock dispersions involved first suspending the desired quantity of NPs in a volume of FBS equivalent to $10 \%$ of the final solution 
volume and sonicating at $1.05 \mathrm{~W}$ for 10 minutes. After sonication, the FBS suspension was diluted to the calculated volume with the desired medium ( $90 \%$ of the total volume) and re-sonicated at $1.05 \mathrm{~W}$ for an additional 20 minutes. These stock dispersions are referred to as either $\mathrm{nZnO}-\mathrm{FBS} /$ water or $\mathrm{nZnO}-\mathrm{FBS} / \mathrm{PBS}$ for stocks prepared in nanopure water or PBS, respectively. Additionally, two nZnO - FBS formulations were created by removing the excess FBS prior to re-suspension and sonication in the appropriate solution. One formulation involved centrifuging the nZnO/FBS suspension after the initial sonication period followed by several washes with nanopure water to remove any unbound FBS. The remaining pellet was then re-suspended in the desired solution to the appropriate

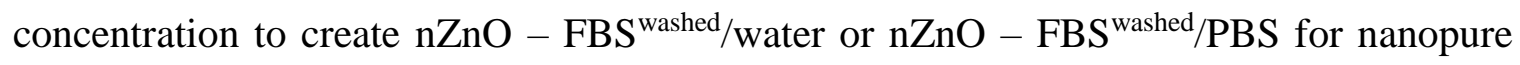
water and PBS dispersions, respectively. Finally, stock dispersions for the dried sample,

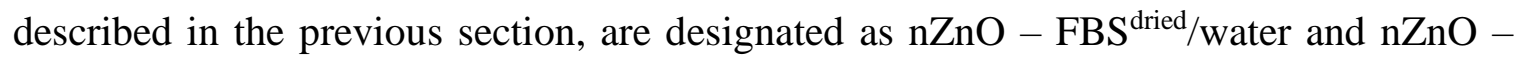

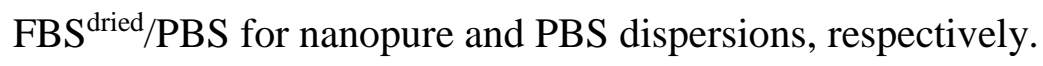

Extracellular Dissolution

For extracellular dissolution experiments, $\mathrm{nZnO}$ stock dispersions were prepared as previously described at $6 \mathrm{mM}$ concentrations. To simulate cellular assay conditions, an aliquot of each stock dispersion was added to cellular media at a final $\mathrm{nZnO}$ concentration of $0.6 \mathrm{mM}$. All dispersions were stirred continuously to prevent $\mathrm{nZnO}$ sedimentation. At desired time points, an aliquot of the sample was removed and centrifuged at 5,000 $\mathrm{x} g$ for 20 minutes using an Amicon ${ }^{\mathrm{TM}}$ Ultra-4 Centrifugal Filter Unit with a 3-Kd molecular weight cutoff ( $0.1 \mathrm{~nm}$ pore size) to remove any undissolved $\mathrm{nZnO}$ while allowing free zinc ions to pass through. Quantitative analysis of the dissolved $\mathrm{Zn}^{2+}$ ions was conducted on a Thermo X Series 2 quadrupole inductively-coupled plasma mass spectrometer (ICP-MS) 
under normal operating conditions (i.e., no CCT) with the $\mathrm{X}_{\mathrm{t}}$ cone set. Instrument performance was evaluated and optimized for each run. The instrument was calibrated using multi-element calibration standards containing $\mathrm{Zn}, \mathrm{Cr}, \mathrm{Mn}, \mathrm{Fe}, \mathrm{Co}$ and $\mathrm{Ni}$ in $2 \%$ $\mathrm{HNO}_{3}$ at concentrations of approximately 1, 10, 100 and $1000 \mathrm{ppb}$. Instrument drift was monitored and corrected using $20 \mathrm{ppb}$ indium as an internal standard introduced online.

\section{Dosimetry Analysis and Empirical Deposition Fractions}

Since NPs suspended in solution often form loosely packed agglomerates consisting of NP clusters and entrapped media, two empirical methods, the Harvard Volume Centrifugation Method (VCM) $[32,33]$ and the $\underline{i} n$ vitro sedimentation, diffusion and dosimetry (ISDD) computational model [43] were employed to determine the effective NP density of the agglomerates $\left(\rho_{e}, \mathrm{~g} / \mathrm{cm}^{3}\right)$, agglomerate porosity parameter ( $\varepsilon_{a g g}$, unitless), diffusion rate $\left(D, \mathrm{~cm}^{2} / \mathrm{s}\right)$, sedimentation velocity $(V, \mathrm{~cm} / \mathrm{s})$, deposition factor, $\left(\alpha, \mathrm{h}^{-1}\right)$, and the deposition fraction curve $\left[f_{D}(t)\right]$. Harvard VCM, which utilizes experimental methods to measure the effective volume of NP pellets $\left(V_{\text {pellet }}\right)$, was employed to estimate the $\rho_{e}$ of the NP agglomerates. To measure the $V_{\text {pellet, }}$ NP suspensions were prepared at a concentration of $100 \mu \mathrm{g} / \mathrm{mL}$ in nanopure water and sonicated with $1.05 \mathrm{~W}$ of power for 30 minutes. The resulting stock dispersions were introduced to RPMI-based cellular media at a concentration of $1.25 \mathrm{mM}$ and then centrifuged at $2,000 \times \mathrm{g}$ for one hour in TPP $($ Techno Plastic Products, Trasadingen, Switzerland) packed cell volume tubes and the resulting NP pellets were measured utilizing the TPP "easy read" measuring device by the same manufacturer.

Once the $\mathrm{V}_{\text {pellet }}$ for a NP dispersion was measured, the $\rho_{e}$ of the NP agglomerates was then calculated using the following equation (DeLoid et al.) [32]: 


$$
\rho_{e}=\rho_{\text {media }}+\left[\left(\frac{M_{Z n O}-M_{\text {ZnoSol }}}{V_{\text {pellet }} S F}\right)\left(1-\frac{\rho_{\text {media }}}{\rho_{\text {Zno }}}\right)\right]
$$

This calculation represents the $\rho_{e}$ as a function of the media density $\left(\rho_{\text {media }}\right)$ and density represented by the NP material. Since $\mathrm{ZnO}$ is partially soluble in nanopure water, the mass of solubilized $\mathrm{ZnO}\left(M_{\text {Znosol }}\right)$ determined by ICP-MS as outlined above was subtracted from the original mass of $\mathrm{ZnO}\left(M_{Z n O}\right)$. A theoretical stacking factor $(S F)$ of 0.634 to approximate random close stacking employed by many dosimetry models $[32,33]$ was utilized. The density value for $\mathrm{ZnO}$ NPs $\left(\rho_{Z n O}\right)$ of $5.606 \mathrm{~g} / \mathrm{cm}^{3}$ was used. The deposition factor, $(\alpha)$ [43], a function of the sedimentation velocity $(V)$, diffusion rate $(D)$, and height of the media in the cell well $(L)$

$$
\alpha=\frac{D}{V L}
$$

was then utilized to generate deposition fraction curves $\left[f_{D}(t)\right]$ :

$$
f_{D}(t)=1-e^{-\propto t}
$$

and time values needed to observe $90 \%$ deposition of suspended NPs in solution ( $\left.t_{90}\right)$ [47]:

$$
t=\frac{-\ln \left(1-f_{D}(t)\right)}{\propto}
$$

To model dosimetry kinetics for suspension cell models, the curves representing the fraction of available NPs $\left[f_{A}(t)\right]$ were created using the following equation:

$$
f_{A}(t)=e^{-\propto t}
$$

To determine the effective density, the in vitro sedimentation, diffusion and dosimetry (ISDD) computational model employs the unitless agglomerate porosity parameter $\left(\varepsilon_{a g g}\right)$ 


$$
\varepsilon_{a g g}=1-\left(\frac{d_{H}}{d_{X R D}}\right)^{D F-3}
$$

where $d_{H}$ represents the hydrodynamic size, $d_{X R D}$ refers to the NP crystalline size measured by XRD, and DF is the specific fractal dimension. A DF value of 2.3, often employed to model metal oxide suspensions [47], was used in this study. Once $\varepsilon_{a g g}$ had been calculated, the $\rho_{e}$ was calculated utilizing equation 7

$$
\rho_{e}=\left(1-\varepsilon_{a g g}\right) \rho_{Z n o}+\varepsilon_{a g g} \rho_{\text {media }}
$$

The $\rho_{e}$ value was then employed to calculate the sedimentation velocity $(V, \mathrm{~m} / \mathrm{s})$ via equation 8

$$
V=\frac{g\left(\rho_{\text {ZnO }}-\rho_{\text {media }}\right) d_{H}{ }^{2}}{18 \mu}
$$

with $\mathrm{g}=9.8 \mathrm{~m} / \mathrm{s}^{2}$ and $\mu$ defined as the media viscosity $(\mathrm{Pa} \cdot \mathrm{s})$. Finally, the diffusion rate $\left(D, m^{2} / s\right)$ was determined by

$$
D=\frac{R T}{3 N_{A} \pi \mu d_{H}}
$$

where $R$ is the gas constant $(L \cdot k P a / K / m o l), N_{A}$ is Avogadro's number and $T(K)$ is the temperature.

\section{Cell Culture and Cytotoxicity Studies}

For cell cytotoxicity assays, Jurkat $\mathrm{T}$ cell leukemia, Hut-78 $\mathrm{T}$ cell lymphoma, T47D epithelial mammary gland carcinoma and the LNCaP epithelial prostate cancer cell line (ATCC, Rockville, MD) were cultured in cellular medium. Additionally, the T-47D media was supplemented with 0.2 units $/ \mathrm{mL}$ bovine insulin. Cells were maintained in log phase at $37{ }^{\circ} \mathrm{C}$ and $5 \% \mathrm{CO}_{2}$, and seeded at a concentration of $5 \times 10^{5}$ cells/well in 96-well plates for Jurkat and Hut-78 cells. For the T-47D and LNCaP cells, the cells were first 
trypsinized using $0.25 \%$ trypsin/ $0.53 \mathrm{mM}$ ethylenediaminetetraacetic acid (EDTA) (MP Biomedicals, LLC; Santa Ana, CA), re-suspended in fresh media and then seeded at a concentration of $2.5 \times 10^{5}$ cells/well in 24 -well plates. The cells were then incubated overnight to allow the cells to re-adhere to the plate. Prior to treatment, the growth medium was gently aspirated from each well and replaced with $400 \mu \mathrm{L}$ of fresh medium. Cells were subsequently treated with freshly sonicated (30-min) NPs reconstituted in the desired solution. Jurkat cells and Hut-78 cells were treated with NPs immediately after plating. For example, for a $2 \mathrm{mg} / \mathrm{ml} \mathrm{ZnO} \mathrm{NP} \mathrm{stock} \mathrm{dispersion,} 3.2 \mathrm{mg}$ of NP were re-suspended in 1.58 $\mathrm{ml}$ of solution medium and sonicated at $50 \mathrm{~W}$ for 30 minutes. Then working dilutions were prepared from the NP stock dispersion and added to $200 \mu \mathrm{l}$ of cell suspensions in $96-$ well plates or $400 \mu \mathrm{l}$ of cell suspensions in 24-well plates and cultured for 24 hours. After 24 hours, the effects of the various NP stock dispersions on cell viability using two different viability assays, alamar blue and flow cytometry. The alamar blue metabolic assay was employed to assess viability based on the fluorogenic redox indicator dye resazurin being converted to the fluorescent resofurin upon reduction by mitochondrial enzymes in metabolically active cells. In this assay, cell populations were seeded into the desired plate at the concentrations indicated above, treated with NPs for 20 hours, and the incubated with alamar blue (10\% of total volume) for an additional 4 hours. Changes in fluorescence were evaluated spectrophotometrically using excitation/emission at 530/590 nm in a Biotek Synergy $\mathrm{MX}^{\circledR}$ plate reader (Winooski, VT).

Flow cytometry was employed as an alternate viability assay to verify experimental results. NP treated cells were dually stained with a fluorescein isothiocyanate-labeled antiHLA ABC antibody and $50 \mu \mathrm{g} / \mathrm{mL}$ propidium iodide (PI) to monitor losses in membrane 
integrity. Fluorescent CountBright counting beads (Invitrogen, Carlsbad, CA) were added to samples to enable determinations of absolute cell numbers, and changes in PI staining were used to quantify cell death. Nanoparticles were excluded from analysis based on absence of fluorescence signal and light forward scatter (FS) and side scatter (SSC) characteristics, and samples were analyzed using a BD FACS Caliber flow cytometer.

For $\mathrm{CD}^{+} \mathrm{T}$ cell cytotoxicity experiments, written informed consent was obtained from all blood donors and the University Institutional Review Board approved this study. Peripheral blood mononuclear cells were obtained from healthy human blood samples via Ficoll-Hypaque density centrifugation (Histopaque-1077, Sigma, St Louis, MO), and $\mathrm{CD}^{+} \mathrm{T}$ cells isolated by negative immunomagnetic selection as previously reported [16]. Purified CD4 ${ }^{+}$T cells (>95 \% purity) were cultured in RPMI/10 \% FBS and suspended at a final concentration of $1 \times 10^{6}$ cells $/ \mathrm{ml}$. CD4 ${ }^{+} \mathrm{T}$ cells were then incubated for 24 hour with increasing amounts of each of the $\mathrm{nZnO}$ suspensions $(24-800 \mu \mathrm{g} / \mathrm{mL})$ in order to establish an $\mathrm{IC}_{50} . \mathrm{CD}^{+} \mathrm{T}$ cells were then stained and viability analyzed by flow cytometry.

\section{$\underline{\text { Reactive Oxygen Species (ROS) Detection }}$}

Mitochondrial superoxide levels were determined by flow cytometry using MitoSOX Red (Invitrogen, Eugene, OR) staining. MitoSOX Red is a cell permeable fluorescent dye that becomes oxidized by interacting with mitochondrial superoxide [48]. The resulting fluorescent signal occurs upon binding of the oxidized dye to nucleic acids. As a positive control, samples were treated with $0.2 \mathrm{nM}$ of the mitochondrial electron transport inhibitor antimycin-A to ensure that the cells were capable of producing ROS. Jurkat cells were incubated with $0.4 \mathrm{mM} \mathrm{nZnO}$ or nZnO-FBS for 6,18 and 24 hours and 
then dually stained with CD3 antibody and $2.5 \mu \mathrm{M}$ MitoSOX Red for $30 \mathrm{~min}$. Mitochondrial superoxide levels were determined by flow cytometry.

$\underline{\text { Statistical Analyses }}$

Statistical analyses were performed using Jump Pro 10 software (SAS; Cary, NC). The cytotoxicity data were analyzed using repeated measures of variance with post hoc comparisons and significance levels defined as $p<0.05$ to determine statistical differences between the means and allow within-sample variation to be separated from betweensample variation. Data for Figure 2.10, Figure 2.11, and Figure 2.12 were analyzed using a two-way analysis of variance (ANOVA) to test for statistical significance of the model, and post hoc comparisons were used to test for statistically significant effects of treatment on cell viability $(p<0.05)$ with an asterisk denoting statistical significance in the indicated figures.

\section{Results and Discussion}

$\underline{\mathrm{ZnO} N a n o p a r t i c l e ~ S y n t h e s i s ~ a n d ~ C h a r a c t e r i z a t i o n ~}$

The pure $\mathrm{ZnO}$ NPs $(\mathrm{nZnO})$ and the FBS-coated formulation $\left(\mathrm{nZnO}-\mathrm{FBS}^{\text {dried }}\right)$ were thoroughly characterized prior to use. Analysis of the powder x-ray diffraction spectra of the NPs revealed the expected hexagonal wurtzite crystal structure (Figure 2.1A). The MAUD software, which employs the Rietveld method [49] showed the particles had an average crystallite size of $10.0 \pm 0.74 \mathrm{~nm}$ and lattice parameters $\mathrm{a}=3.2580 \AA$ and $\mathrm{c}=5.226$ A. The $\mathrm{nZnO}-\mathrm{FBS}^{\text {dried }}$ sample also showed similar crystallite size and lattice parameters suggesting that the FBS coating did not alter the crystalline structure of the nZnO nanoparticles. Additionally, TEM analysis confirmed that the NPs in both formulations were primarily spherical in morphology with similar size distributions (Figure 2.1B, C). 
Surface charge for $\mathrm{nZnO}$ and the $\mathrm{nZnO}-\mathrm{FBS}^{\text {dried }} \mathrm{NP}$ samples, measured in nanopure water, were found to be $43.8 \pm 0.55 \mathrm{mV}$ and $-11.8 \pm 0.39 \mathrm{mV}$, respectively. Bovine serum albumin (BSA), the predominant component of FBS has an isoelectric point (IEP) of 5.1 indicating that BSA would be positively charged at lower $\mathrm{pH}(<5.1)$ and negatively charged at higher $\mathrm{pH}$ values (>5.1) [50]. Therefore, the positive to negative shift in the zeta potential observed for the $\mathrm{nZnO}-\mathrm{FBS}^{\text {dried }}$ NPs is indicative of the absorption of negatively charged protein components on the surface of the $\mathrm{nZnO}$ [51]. To determine the IEP of each NP suspension, the $\mathrm{pH}$ was varied from 3-12 using $0.1 \mathrm{M} \mathrm{HCl}$ or $0.1 \mathrm{M}$ $\mathrm{NaOH}$ and the zeta potential was measured at several points in three independent trials (Figure 2.2A). An IEP of 9.5 was observed for $\mathrm{nZnO}$ indicating these NPs would carry a positive charge below $\mathrm{pH} 9.5$ and a negative charge at $\mathrm{pH}>9.5$. In contrast, the $\mathrm{nZnO}-$ FBS $^{\text {dried }}$ formulation had an IEP of $\mathrm{pH}$ 6.1, resulting in positively charged particles at much lower $\mathrm{pH}$ values (below 6.1) and negatively charged particles at $\mathrm{pH}>6.1$. Below $\mathrm{pH} 6.5$, partial NP dissolution was observed for $\mathrm{nZnO}$ but not for the $\mathrm{nZnO}-\mathrm{FBS}^{\text {dried }}$ sample. The decreasing zeta potential trend observed at low $\mathrm{pH}$ values for $\mathrm{nZnO}$ has been documented for $\mathrm{nZnO}$ prepared using this synthesis method [28].

The FTIR spectra of $\mathrm{nZnO}$ and $\mathrm{nZnO}-\mathrm{FBS}^{\text {dried }}$ are shown in Figure 2.2B. Both samples contain a strong band characteristic of $\mathrm{Zn}-\mathrm{O}$ vibrations [52] at $456 \mathrm{~cm}^{-1}(\mathrm{nZnO})$ and $451 \mathrm{~cm}^{-1}\left(\mathrm{nZnO}-\mathrm{FBS}^{\text {dried }}\right)$. The $\mathrm{nZnO}$ sample exhibits two bands commonly associated with the carboxyl functional group, specifically $1412 \mathrm{~cm}^{-1}\left[v_{\mathrm{s}}\left(\mathrm{COO}^{-}\right)\right]$and 1597 $\mathrm{cm}^{-1}\left[v_{\mathrm{as}}\left(\mathrm{COO}^{-}\right)\right][53,54]$. Additionally, weaker bands consistent with $\tau\left(\mathrm{CH}_{2}\right)$ and $v(\mathrm{C}-\mathrm{OH})$ at 903 and $1076 \mathrm{~cm}^{-1}$, respectively, indicate the presence of surface-adsorbed carboxyl groups originating from the zinc acetate precursor or the DEG solvent [52-54]. 
Furthermore, the absence of the $v(\mathrm{C}=\mathrm{OOH})$ band at $\sim 1720 \mathrm{~cm}^{-1}$ suggests that the carboxyl groups are in an expected deprotonated form as a result of the alkaline synthesis procedure [53]. Noticeably absent in the $\mathrm{nZnO}-\mathrm{FBS}^{\text {dried }}$ samples are the strong symmetric and asymmetric stretching modes of the carboxyl group with new bands appearing in the spectrum at $1646 \mathrm{~cm}^{-1}$ and $1533 \mathrm{~cm}^{-1}$ representing the amide I and amide II regions that arise from vibrations of the peptide bonds of the protein backbone $[55,56]$. The amide I region, often used for secondary structure prediction, exhibits a maximum at 1650- 1656 $\mathrm{cm}^{-1}$ when $\alpha$-helices predominate in the protein secondary structure [56]. At $1646 \mathrm{~cm}^{-1}$, the amide $\mathrm{I}$ band of the $\mathrm{nZnO}-\mathrm{FBS}^{\text {dried }}$ particles is close to this wavenumber maximum and suggestive of the presence of a protein with the $\alpha$-helical structure components found in BSA. The slight shift of this maximum is consistent with a more random secondary structure (1644-1648 $\left.\mathrm{cm}^{-1}\right)$ and has been observed in protein adsorption and binding studies with albumin [57]. Taken together, these findings clearly establish the displacement of adsorbed carboxyl groups and strong binding of the FBS protein components $[58,59]$ on the nanoparticle surface.

\section{$\underline{\text { Agglomeration and Sedimentation Profiles }}$}

As previously discussed, determining the best media components and dispersion conditions for the preparation of stable $\mathrm{nZnO}$ dispersions was a major goal of this study. Reported cellular toxicity studies using Jurkat and Hut-78 cells have frequently employed a variety of different biological solutions used to disperse NP powder samples prior to treatment. Considerations in choosing stock solution components include maintaining a viable environment for the cells as well as creating a stable NP dispersion for treatment conditions. Commonly used stock solutions consist of nanopure water, PBS or un- 
supplemented cellular media. In addition, various capping agents [60] or protein components are often utilized to reduce steric attractions between charged particles and solution components and reduce NP agglomeration [51, 60, 61]. Solutions such as PBS or un-supplemented media are generally preferred as they do not result in unwanted $\mathrm{pH}$ changes during the toxicity study or affect cellular osmolality. Likewise, media proteins such as BSA and FBS are frequently used as stabilizing agents.

The average hydrodynamic size of the $\mathrm{nZnO}$ sample in six different $25 \mathrm{mM}$ stock dispersions was measured every 10 minutes for a 24-hour time period using dynamic light scattering (DLS) technique (Figure 2.3A). From this data, inferences can be made about the agglomeration tendencies of the NPs over time in different biological solution systems. Two of the solutions, RPMI and PBS, exhibited unstable average hydrodynamic size profiles over time evidenced by the immediate formation of very large agglomerates of 2,031 $\mathrm{nm}$ and 3,578 $\mathrm{nm}$, respectively. The agglomerate sizes in PBS increased over time from $3,578 \mathrm{~nm}$ to $5,811 \mathrm{~nm}$ and eventually stabilized to a variable range of $2,500-4,200$ $\mathrm{nm}$ over the remaining test period. In RPMI, the $\mathrm{nZnO}$ agglomerate size increased dramatically over time from $2,031 \mathrm{~nm}$ to sizes exceeding the limits of the Zetasizer $(\sim 10,000 \mathrm{~nm})$ by the end of the time period. These data indicate that agglomeration behavior of NP systems increases with increasing ionic strength of the dispersion solution (nanopure water < PBS < RPMI). All other test dispersions exhibit stable average hydrodynamic size profiles over time with average size profiles of $306 \mathrm{~nm}$ (nanopure water), 150-200 nm (DMEM-based cellular media), $263 \mathrm{~nm}$ (RPMI-based cellular media) and $78 \mathrm{~nm}(\mathrm{FBS})$. To evaluate the effect of FBS on dispersion stability, all three $\mathrm{nZnO} / \mathrm{FBS}$ formulations, $\mathrm{nZnO}-\mathrm{FBS}^{\text {dried }}$ (dried FBS-coated $\mathrm{nZnO}$ ), $\mathrm{nZnO}-\mathrm{FBS}^{\text {washed }}$ (FBS-coated 
nZnO with excess FBS removed) and $\mathrm{nZnO}-\mathrm{FBS}$ (nZnO coated with $10 \%$ FBS per volume of desired solution), were dispersed in both PBS and nanopure water at concentrations of $25 \mathrm{mM}$. For PBS stock dispersions, the FBS-coating provided long-term hydrodynamic size stability (up to $\sim 15$ hours) regardless of the formulation employed when compared to the nZnO/PBS dispersion (Figure 2.3B). Beyond 15 hours, the $\mathrm{nZnO}-$

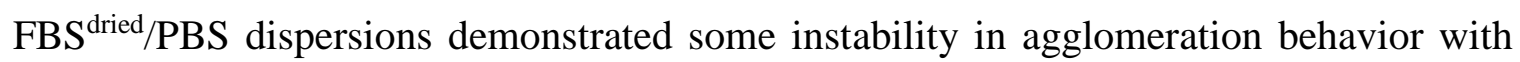
average agglomerate sizes increasing from approximately $400 \mathrm{~nm}$ to $800 \mathrm{~nm}$. The addition of FBS in nanopure water dispersions did not appreciably change the agglomerate stability of the NP suspensions. Relatively stable agglomeration profiles were observed between $\sim 200-350 \mathrm{~nm}$ for all dispersions (Figure 2.3C). Interestingly, the $\mathrm{nZnO}-\mathrm{FBS}^{\mathrm{dried}}$ formulation also displayed a mild degree of dispersion instability at $\sim 13$ hours. These findings, along with those for the PBS-based suspension, suggest that the $\mathrm{nZnO}-\mathrm{FBS}^{\mathrm{dried}}$ formulation is not the most ideal preparation for downstream applications which require long-term dispersion stability.

Although average size profiles highlight the agglomeration tendencies of NP dispersions, they fail to depict how agglomerate formation affects NP sedimentation, changes in the net $\mathrm{nZnO}$ concentration in solution over time and the ever-changing size distribution of a complex NP dispersion. To address these shortcomings, experiments evaluating the sedimentation behavior of $\mathrm{nZnO}$ dispersions were performed by measuring changes in the optical absorbance intensity at $\lambda_{\max }(\sim 378 \mathrm{~nm}$ corresponding to the band gap energy of $\mathrm{nZnO}$ ) relative to the initial absorbance intensity. Decreases in the relative absorbance intensity at $\lambda_{\max }$ as a function of time have been correlated to decreasing NP concentrations in solution as a result of NP sedimentation [23, 62]. The sedimentation 
tendencies over a two-hour time period for various stock dispersions are depicted in Figure 2.4A. As expected, nZnO in RPMI rapidly settles out of solution with $100 \%$ sedimentation noted by the one-hour time point. Other dispersions that displayed sedimentation behavior were $\mathrm{nZnO}$ dispersions in both RPMI-based cellular media (29\% at 2 hours) and PBS (49 $\%$ at 2 hours). None of the $\mathrm{nZnO}-\mathrm{FBS}$ formulations $\left(\mathrm{nZnO}-\mathrm{FBS}^{\text {dried }}, \mathrm{nZnO}-\mathrm{FBS}^{\text {washed }}\right.$ or $\mathrm{nZnO}-\mathrm{FBS}$ ) showed any appreciable sedimentation behavior over the 2-hour time period in PBS or nanopure water (Figure 2.4A, B).

Further evidence of NP dispersion stability can be observed in the hydrodynamic size distribution profiles at various time intervals (Figure 2.5, 6 and 7). Stable dispersions, such as those observed for the nanopure water (Figure 2.5A), demonstrate similar size distribution trends across the evaluated time period. Strong agglomeration and sedimentation tendencies result in broader distributions initially as a result of immediate agglomerate formation, followed by narrower distributions as large agglomerates settle out of the dispersion. This trend is readily apparent for the PBS dispersion (Figure 2.5B) and present to a lesser degree for the cellular media stock (Figure 2.5C) which reflects the improved stability of the $\mathrm{nZnO}$ in RPMI-based cellular media, containing $10 \% \mathrm{FBS}$, compared to the PBS stock. The size distribution trends observed for the $\mathrm{nZnO}-\mathrm{FBS}^{\text {dried }}$, $\mathrm{nZnO}-\mathrm{FBS}^{\text {washed }}$ and $\mathrm{nZnO}$ - FBS dispersions in PBS (Figure 2.6) and water (Figure 2.7) affirm the findings previously observed. Both the $\mathrm{nZnO}-\mathrm{FBS}^{\text {dried}} / \mathrm{PBS}$ (Figure 2.6A) and

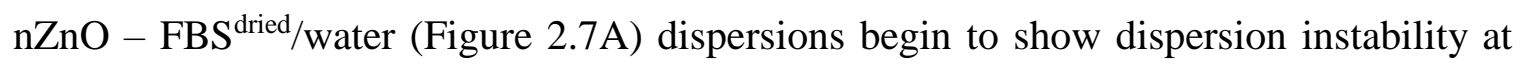
$\sim 12$ hours which is readily apparent by 24 hours. Taken together, the agglomeration profiles obtained from DLS and the sedimentation trends observed by UV-Vis 
spectrophotometry provide an accurate qualitative assessment of the NP dispersion stability.

After evaluating several stock dispersions independently, the behavior of the NPs was assessed under conditions used for cellular toxicity assays. In short, aliquots of the 25 $\mathrm{mM} \mathrm{nZnO} / \mathrm{PBS}$ and $\mathrm{nZnO}$ - FBS/PBS stock dispersions were introduced to RPMI-based cellular media at concentrations of $0.6 \mathrm{mM}$ and the agglomeration and sedimentation tendencies of the NPs were observed for 24 hours (Figure 2.8). Analysis of the data collected under in vitro experimental conditions reveal that NPs dispersed in nZnO/PBS display strong agglomeration potential in the cellular media compared to those prepared using the $\mathrm{nZnO}-\mathrm{FBS} / \mathrm{PBS}$ stock. NPs in the dispersion prepared using the PBS stock formed large agglomerates immediately (>500 nm) (Figure 2.8A, C), which precipitated rapidly from the cellular media (Figure 2.8B), reducing the percent intensity and changing the maximum in the agglomerate size distribution (Figure 2.8C). At the 12-hour time point, the hydrodynamic size profile revealed a large distribution of NP sizes present within solution with a $54 \%$ decrease in the $\mathrm{nZnO}$ absorbance. Significant decreases in the hydrodynamic size distribution can be seen by the 24-hour time point suggesting that large agglomerates had precipitated from the cellular media (Figure 2.8A and C). The sedimentation profile (Figure 2.8B) confirms that by 24 hours nearly $60 \%$ of the nZnO was no longer present in the cellular media. Comparatively, the experimental dispersion (0.6 mM) prepared from the $\mathrm{nZnO}-$ FBS/PBS stock exhibited considerably more size stability over time (Figure 2.8A and D) resulting in less sedimentation behavior (39\%) by 24 hours (Figure 2.8B). In the context of in vitro cytotoxicity studies, these results imply that using FBS as a NP stabilizing agent prior to solution dispersion results in more NPs 
being bioavailable to interact with cells cultured in suspension. Although most of the $\mathrm{ZnO}$ NPs are in the $100-250 \mathrm{~nm}$ range at the 24 -hour time point, there are some significantly large agglomerates $(3,000-6,000 \mathrm{~nm})$ in the hydrodynamic size profiles (inset in Figure 2.8D) of $\mathrm{nZnO}$ - FBS samples. However, their percent abundance is $<5 \%$ of the total number of observed aggregates at 24 hours.

\section{$\underline{\text { Dissolution Studies }}$}

Experiments were conducted to investigate if $\mathrm{Zn}^{2+}$ ions are released from the $\mathrm{ZnO}$ NP, when dispersed in stock solutions prepared at concentrations typically employed for downstream biological assays $(6 \mathrm{mM}$ of $\mathrm{nZnO}) . \mathrm{Zn}^{2+}$ dissolution was measured using a PBS stock dispersion and compared to the same after adding $10 \%$ FBS to improve dispersion stability. Figure 2.9 shows the $\mathrm{Zn}^{2+}$ dissolution data (displayed as percentage of the total $\mathrm{nZnO}$ concentration in each solution as a function of time). Among these, the $\mathrm{nZnO}-\mathrm{FBS} / \mathrm{PBS}$ stock dispersion showed the highest $\mathrm{Zn}^{2+}$ dissolution $(0.151 \pm 0.029 \%$ at 24 hours $)$ while the $\mathrm{nZnO} / \mathrm{PBS}$ stock $(0.033 \pm 0.004 \%$ at 24 hours $)$ showed significantly less $\mathrm{Zn}^{2+}$ ion release. These dissolution studies were then extended to samples prepared under actual experimental conditions $(0.6 \mathrm{mM} \mathrm{nZnO})$ used for the in vitro toxicity assay studies as previously described. This lowering of the $\mathrm{nZnO}$ concentration by 10 -fold caused significant increases in percentage $\mathrm{Zn}^{2+}$ dissolution for both dispersion combinations, as shown in Figure 2.9. Decreasing $\mathrm{nZnO}$ concentration in solution will decrease NP agglomeration tendencies and subsequently increase the interaction of the nanoparticles with the medium, resulting in more $\mathrm{Zn}^{2+}$ ion formation in the dispersion [63]. Interestingly,

the FBS/PBS sample showed lower $\mathrm{Zn}^{2+}$ dissolution $(8.668 \pm 0.247 \%$ at 24 hours) than the pure PBS sample $(10.041 \pm 2.905 \%$ at 24 hours $)$. Since FBS addition increases 
dispersion stability and reduces hydrodynamic size, an increase in the $\mathrm{Zn}^{2+}$ dissolution is expected if interaction of the NPs with the dispersed medium alone is the most important factor. The observed lower $\mathrm{Zn}^{2+}$ dissolution of the $\mathrm{nZnO}$ - FBS/PBS sample may be due to the presence of serum proteins from FBS on the $\mathrm{nZnO}$ surface, reducing direct NP-media interaction and thus protecting them from $\mathrm{Zn}^{2+}$ dissolution. Both FTIR and zeta potential measurements have shown that $10 \%$ FBS addition produces a surface layer of serum proteins on the $\mathrm{nZnO}$ surface. Thus, the lower $\mathrm{Zn}^{2+}$ dissolution of $\mathrm{nZnO}-\mathrm{FBS} / \mathrm{PBS}$ dispersion $(0.6 \mathrm{mM})$ is likely attributed to the serum coating of the $\mathrm{nZnO}$ and the resulting reduction in the NP-medium interactions. Another potential contributing factor in these systems is the propensity of free zinc ions to form insoluble zinc carbonate $\left(\mathrm{ZnCO}_{3}\right)$ or zinc phosphate $\left(\mathrm{Zn}_{3}\left(\mathrm{PO}_{4}\right)_{2}\right)$ precipitates in biologically relevant solutions [61, 64]. Precipitation of these species may explain dissolution potential differences between the prepared $\mathrm{nZnO} / \mathrm{PBS}$ and $\mathrm{nZnO}$ - FBS/PBS stock dispersions given the assumption that the FBS present in suspension may provide a protective function against $\mathrm{nZnO}$ and $\mathrm{Zn}^{2+} /$ media interactions, thus reducing the amount of insoluble complexes formed and increasing the concentration of ionic $\mathrm{Zn}^{2+}$ [65]. However, if this were the sole contributing factor, the same dissolution pattern would be expected when the stock dispersions were introduced into the cellular media. The reversal of the dissolution potential trend going into the cellular media underscores the complex nature of $\mathrm{nZnO}$ dissolution in biologically relevant solutions. While our results appear to contradict a recent study by Hsiao and Huang [41] in which a much larger $\mathrm{Zn}^{2+}$ dissolution was observed when FBS-coated $\mathrm{ZnO}$ NPs were dispersed in DMEM media, these apparent discrepancies may be attributable to solutionrelated dissolution differences. Additional studies conducted in our laboratory have shown 
that $\mathrm{nZnO}$ dispersed in the RPMI-based cellular media employed in this study exhibit significantly less dissolution potential than $\mathrm{nZnO}$ dispersed in the DMEM used by Hsiao and Huang (data not shown). The decrease in ionic zinc in the RPMI-based media compared to DMEM could be attributed to increased precipitation of insoluble $\mathrm{Zn}_{3}\left(\mathrm{PO}_{4}\right)_{2}$ in the phosphate-rich RPMI system [61, 65]. Furthermore, NP dissolution in the presence of serum proteins appears to be a competition between two processes. Serum proteins reduce agglomerate size allowing more interaction between NP surface and the dispersing medium. However, serum proteins will also attach to the nanocrystal surface effectively reducing the interaction of surface bound $\mathrm{Zn}$ ions and the medium.

\section{$\underline{\text { Reactive Oxygen Species (ROS) }}$}

The potential role of intracellular ROS generation in the cytotoxicity of $\mathrm{nZnO}$ was demonstrated in our earlier reports $[10,15,16]$ and through several other studies for a variety of cells types [66-68] by using fluorescent dyes to directly detect ROS. Since redox reactions at the NP surface are a primary driver in extracellular and intracellular ROS production, changes in the NP surface structure as a result of protein coating could change ROS generation and, in turn, also influence the cytotoxicity. To evaluate the amount of ROS produced by $\mathrm{nZnO}$ and $\mathrm{nZnO}-\mathrm{FBS}$, Jurkat cells were exposed to $0.4 \mathrm{mM} \mathrm{ZnO}$ for 6 , 18 and 24 hours and subsequently stained with MitoSOX Red. As Figure 2.10 illustrates, appreciable differences exist between the amounts of ROS produced at 6 hours (Figure 2.10A and B), as measured by the mean MitoSOX fluorescence intensity (MFI), between the $\mathrm{nZnO}$ dispersion (PBS stock; $33.9 \pm 2.66 \mathrm{MFI}$ ) versus the $\mathrm{nZnO}-\mathrm{FBS}$ dispersion (FBS/PBS stock; $49.9 \pm 4.98 \mathrm{MFI}$ ) representing a $47.2 \%$ increase in the amount of NPinduced ROS present in cells treated with the $\mathrm{nZnO}-\mathrm{FBS}$ dispersion. As the exposure time 
increases, the differences in the ROS production increase significantly. The greatest disparity, noted at 24 hours (Figure 2.10A and D), represents an $88.2 \%$ increase in the amount of ROS induced by the nZnO-FBS over the $\mathrm{nZnO}$ dispersion. Given that the agglomerates present in the nZnO-FBS dispersion are significantly smaller and more bioavailable than those in the $\mathrm{nZnO}$ dispersion, more surface area to volume interactions are possible in the more stable nZnO-FBS which could result in the significant differences observed in the ROS induction. While many surface modification, such as $\mathrm{SiO}_{2}$ coating have demonstrated protective benefits such as less cytotoxicity and cellular stress-inducing ROS production $[42,66]$, FBS-coating does not appear to impart protective benefits against ROS generation.

\section{Cellular Toxicity Studies}

It has been previously shown in our laboratory that these $\mathrm{nZnO}$ synthesized in DEG media demonstrate strong cytotoxic effects against Jurkat leukemic and Hut-78 lymphoma T cell cancer lines [16]. Experiments were performed to determine if FBS coating and the resulting improvements in NP stability and bioavailability influence the cytotoxicity of NPs in these suspension cell models. Cells were exposed to increasing concentrations of freshly prepared nZnO/PBS or nZnO - FBS/PBS stock dispersions for 24 hours. NPinduced toxicity was then determined using an alamar blue assay or through flow cytometry utilizing a T-cell specific fluorescent antibody and propidium iodide (PI) dye uptake [16]. As shown in Figure 2.11, both types of cancer cells showed a significant increase in the NP-induced cytotoxicity $(p=0.0022$ and $p=0.0488$ for Jurkat and Hut-78 cells, respectively) when $\mathrm{nZnO}$ - FBS/PBS stock treated cultures were employed, thus confirming the favorable role of $10 \%$ FBS addition in the stock dispersion preparation. 
The $\mathrm{IC}_{50}$ values for Jurkat cells decreased from $0.39 \pm 0.03 \mathrm{mM}$ for $\mathrm{nZnO} / \mathrm{PBS}$ dispersions to $0.27 \pm 0.02 \mathrm{mM}$ for the $\mathrm{nZnO}-\mathrm{FBS} / \mathrm{PBS}$ dispersion using alamar blue staining (Figure 2.11A) and from $0.38 \pm 0.03 \mathrm{mM}$ for uncoated $\mathrm{nZnO}$ to $0.25 \pm 0.004 \mathrm{mM}$ for $\mathrm{nZnO}-\mathrm{FBS}$ using flow cytometry and PI uptake (Figure 2.11B). Similarly, the $\mathrm{IC}_{50}$ value for the Hut cells (Figure 2.11C) also showed a decrease from $0.21 \pm 0.03 \mathrm{mM}$ when treated with the $\mathrm{nZnO} / \mathrm{PBS}$ dispersion to $0.14 \pm 0.02 \mathrm{mM}$ when treated with the $\mathrm{nZnO}-\mathrm{FBS} / \mathrm{PBS}$ dispersion. These results clearly demonstrate that coating nZnO with FBS proteins and the resulting improved dispersion stability increases their toxic response to cancer cells by $\sim 33 \%$ for both cell types. Additionally, FBS coatings may also provide a potential approach to reduce the $\mathrm{IC}_{50}$ values of $\mathrm{nZnO}$ against cancer cells from their current $\mathrm{mM}$ range to more suitable ranges appropriate for therapeutic applications.

Our previous work has shown that ZnO NPs display some selectivity in their toxic response against cancerous $\mathrm{T}$ cells as the $\mathrm{IC}_{50}$ values observed for primary quiescent $\mathrm{T}$ cells under similar conditions are considerably higher. Similar toxicity studies using nZnO FBS/PBS dispersions also showed stronger toxic responses $(p=0.0303)$ to resting $\mathrm{CD}^{+}$ T lymphocytes (Figure 2.11D). Here, the $\mathrm{IC}_{50}$ values decreased from $4.69 \pm 0.85 \mathrm{mM}$ when treated with $\mathrm{nZnO} / \mathrm{PBS}$ dispersion to $2.43 \pm 0.0 .24 \mathrm{mM}$ when $\mathrm{nZnO}-\mathrm{FBS} / \mathrm{PBS}$ dispersions were used. Thus, the effect of coating NP with serum proteins is evident in primary cells as well. While these results are consistent with our previously reported preferential cytotoxicity of $\mathrm{nZnO}$ to cancer cells, the therapeutic index of Hut cancer cells decreased from 22.6 for $\mathrm{nZnO} / \mathrm{PBS}$ dispersions to 17.0 for the $\mathrm{nZnO}-\mathrm{FBS} / \mathrm{PBS}$ dispersion. Even with the modest decrease noted in the therapeutic index for the $\mathrm{nZnO}$ FBS/PBS dispersion, the resulting therapeutic efficiency of the $\mathrm{nZnO}$ appears better than 
some commonly recognized traditional chemotherapeutic agents using comparable in vitro assays [16]. Thus, the protein interaction with $\mathrm{nZnO}$ increases their cytotoxicity in the suspension cell models evaluated, yet maintains some of the cancer cell selectivity previously observed.

Some published studies have reported that coating $\mathrm{nZnO}$ with organic molecules or media protein components results in decreased cytotoxicity and ROS accumulation compared to uncoated $\mathrm{nZnO}[25,38,40-42,69]$. While these studies appear to contradict our findings, it should be noted that most of these reports evaluated adherent cells while the primary $\mathrm{T}$ cells and cancerous counterparts employed here are all grow as suspension cultures. In adherent cell cultures, poor dispersion stability results in faster sedimentation of NP agglomerates onto the cell monolayer. This rapid sedimentation may artificially lead to higher increases in NP-cell interactions [34] and the observed increased toxicity [39, 41, 42]. When the dispersion stability improves and sedimentation decreases with the addition of suitable molecules such as FBS, adherent cells will be exposed to lesser amounts of deposited NPs resulting in more accurate dosimetry kinetics, measured effective administered doses and observed toxicity values, as reported by many recent studies [25, 38, 40-42, 69]. This possibility was confirmed by comparing the effects of nZnO/PBS and $\mathrm{nZnO}$ - FBS/PBS dispersions in two different adherent epithelial cancer cell lines, T-47D mammary gland carcinoma and LNCaP prostate cancer cell lines (Figure 2.12). For both adherent cell lines, the nZnO - FBS/PBS treated cells exhibited less toxicity when compared to the nZnO/PBS treated cells $(p=0.0020$ and $p=0.0008$ for the T-47D and LNCaP cells, respectively). For the T-47D (Figure 2.12A) cells, a $48.7 \%$ increase in the $\mathrm{IC}_{50}$ was observed for the $\mathrm{nZnO}-\mathrm{FBS} / \mathrm{PBS}$ dispersions over the $\mathrm{nZnO} / \mathrm{PBS}$ treated cells 
(from $0.39 \pm 0.02 \mathrm{mM}$ for $\mathrm{nZnO} / \mathrm{PBS}$ to $0.58 \pm 0.01 \mathrm{mM}$ for $\mathrm{nZnO}-\mathrm{FBS}$ ). Comparatively, the $\mathrm{IC}_{50}$ for the LNCaP cells (Figure 2.12B) increased from $0.35 \pm 0.02 \mathrm{mM} \mathrm{nZnO/PBS}$ treated cells to $0.60 \pm 0.06 \mathrm{mM}$ for $\mathrm{nZnO}-\mathrm{FBS} / \mathrm{PBS}$ treated cells representing a $71.4 \%$ decrease in the toxic response.

In comparison to the suspension cell models, FBS-coating leads to opposite effects in adherent cells. Suspension cells interact with fewer NPs in experimental conditions consisting of even minimally unstable dispersions since a portion of the original NP concentration sediments and may not be bioavailable for cellular interactions. This will skew toxicity assay results to suggest lower toxicity for suspension cells than expected. When the stability of the NP dispersion is improved by suitable molecular additives such as $10 \%$ FBS (Figure 2.11), the cytotoxicity increases significantly for both Jurkat and Hut cancer cells as well as for the primary $\mathrm{T}$ cell lymphocytes. More ZnO NPs are freely available in the stable NP dispersions to interact with more cells, thus resulting in the higher cytotoxicity observed in suspension cell models. The stability-induced differences between suspension and adherent cells underscore the importance of this work. The results from suspension cell studies alone would seem surprising considering other reports employing similar serum coatings describe decreases in NP-induced cytotoxicity in adherent cells lines suggesting that NP coating could be a potential means to control unwanted cytotoxicity [39]. One such study, Hsiao and Huang [41], demonstrated that 5-10\% FBS coating on $\mathrm{ZnO}$ NPs increased dispersion stability and $\mathrm{Zn}^{2+}$ ion release, and decreased the NP-induced cytotoxicity. They hypothesized that this reduction in cytotoxicity in the serum coated NPs was a result of serum-induced increases in cell growth and resulting decreases in $\mathrm{nZnO}$ dosage-per-cell ratios. Additionally, these authors [41] showed larger $\mathrm{Zn}^{2+}$ release 
in their experiments with FBS coated $\mathrm{ZnO}$ thus negating $\mathrm{Zn}^{2+}$ release as a primary contributing mechanism to $\mathrm{nZnO}$ cytotoxicity. In this present study, FBS coating appears to decrease the $\mathrm{Zn}^{2+}$ release while significantly increasing NP-induced cytotoxicity for the suspension cell model when compared to uncoated $\mathrm{nZnO}$. With the addition of the $10 \%$ FBS to our phosphate-rich stock dispersion, it is plausible that decreases in formation of toxic insoluble $\mathrm{Zn}_{3}\left(\mathrm{PO}_{4}\right)_{2}$ precipitates [70] could translate to perceptible decreases in the NP-induced cytotoxicity observed for $\mathrm{nZnO}-\mathrm{FBS} / \mathrm{PBS}$ treatment. However, decreases in $\mathrm{Zn}_{3}\left(\mathrm{PO}_{4}\right)_{2}$ formation would also result in measured increases in $\mathrm{Zn}^{2+}$ release which was not observed. Therefore, extracellular $\mathrm{Zn}^{2+}$ dissolution is unlikely to be a primary factor for the increased cytotoxicity noted for our nZnO - FBS NPs. It is clear from FTIR and zeta potential studies that FBS molecules bind to the $\mathrm{ZnO}$ NP surface. This modification of the NP surface influences their interaction with other NPs (and on agglomerate formation and sedimentation effects) as well as with cells.

\section{$\underline{\text { Modeling and Dosimetry Studies }}$}

Given the toxicity trend differences noted for the differing cell models, mathematical models were investigated as a potential method to confirm the observed trends and to model the dosimetry kinetics for both the investigated stock dispersions (nZnO/PBS versus nZnO - FBS/PBS) and the different cellular models. All of the investigated experimental parameters of dispersion stability can be applied to the $\underline{i n}$ vitro sedimentation, diffusion and dosimetry (ISDD) computational models, put forth by Hinderliter et al. (2010), to predict the transport properties of the NPs in various experimental conditions [43]. The NP and experimental parameters which most influence the NP transport properties include hydrodynamic size $\left(d_{h}\right)$, agglomeration state, effective 
particle density $\left(\rho_{e}\right)$ and the media height in the cell well $(L)$ [43]. Central to the effective dosimetry determination in the ISDD model is the calculation of a NP deposition factor $\alpha$ $\left(\mathrm{h}^{-1}\right)$, Equation 2 in "Methods", which interrelates the diffusion rate $(D)$, sedimentation velocity $(V)$ and media height $[32,33,43,44]$. While the diffusion rate is primarily dependent upon the hydrodynamic size, the sedimentation velocity depends upon both the hydrodynamic size and the effective particle density occupied by NP agglomerates [43, 44]. The effective particle density $\left(\rho_{e}\right)$ can be approximated in the ISDD model (Equation 7) using the hydrodynamic size-dependent agglomerate porosity parameter $\left(\varepsilon_{a g g}\right)$ [43] or can be determined experimentally (Equation 1) as a part of the Harvard Volume Centrifugation Method (VCM) [33, 43]. The Harvard VCM employs an experimental determination of NP agglomerate volumes to directly determine effective particle density. Furthermore, the VCM has been modified to incorporate mass lost due to the dissolution of soluble or partially soluble NPs such as $\mathrm{ZnO}[32,33]$.

To determine dosimetry curves for various experimental conditions, $25 \mathrm{mM}$ dispersions were prepared and dispersed in complete cellular media at a concentration of $1.25 \mathrm{mM}$ and the effective density $\left(p_{e}\right)$ determined using Harvard VCM (Table 2.1) [33]. From the calculated deposition factor, $\alpha$, dosimetry curves were constructed (Figure 2.13) as described in Cohen et al. (2014) [33]. Originally introduced as a model for adherent cell culture, the deposition fraction curve $\left[f_{D}(t)\right]$ (Equation 3; Figure 2.13A) depicts the fraction of NPs that are deposited upon an adherent cell layer as a function of time $[32,33]$. However, the same $\alpha$ values may be used to model the fraction of NPs that remain bioavailable $\left[f_{A}(t)\right]$ as a function of time for suspension cells (Equation 5; Figure 2.13B). Based on these curves, a t90 value can be calculated to make accurate determinations of 
effective NP dose either deposited on an adherent cell layer or bioavailable to suspension cells at desired time points.

The influence of FBS on the dosimetry kinetics is evident in an analysis of the experimentally stable water, FBS/water and FBS stock dispersions introduced to the RPMI-based cellular media. Given the dependence of agglomerate behavior and effective density $\left(p_{e}\right)$ on the hydrodynamic size of NPs in dispersion, the water $(182.5 \mathrm{~nm})$, FBS/water (185.1 nm) and FBS (185.5 nm) stock dispersions should exhibit similar dosimetry kinetics. However, the effective density $\left(p_{e}\right)$ of the NPs in the three dispersions decreased with increasing amounts of FBS in the stock preparation thus increasing the calculated deposition factor. These disparities are a direct result of measured increases in the effective volume of the NP pellet with increasing amounts of FBS in solution. So while the similarities in the hydrodynamic diameters of these three dispersions imply consistent NP diffusion rates (Equation 9), the differing effective volume measurements suggest different NP sedimentation velocities (Equation 8) [32]. Therefore, even though all three dispersions demonstrate dispersion stability experimentally, the effective volume occupied by the resulting protein corona in the NP agglomerates decreases the sedimentation velocities of the NPs predicted by the model, thus increasing the deposition factor and shifting the dosimetry kinetics.

Interestingly, the dose metrics obtained for the unstable stock dispersions of PBS and RPMI suggest dosimetry trends opposite to what would be expected considering the experimentally determined agglomeration and sedimentation profiles. However, the VCM dosimetry model assumes that the dispersions used to obtain the experimental $\alpha$ values are stable across the time frames defined by the model. As this is not the case with the PBS 
and RPMI stock dispersions, these obtained dosimetry curves cannot be used to accurately model the dosimetry kinetics under these conditions. To model potentially unstable dispersions, it is necessary to calculate $\alpha$ values using experimentally determined stability parameters such as hydrodynamic size at multiple time points. For this, we employed the ISDD model. This model approximates effective particle density utilizing the hydrodynamic size-dependent parameter $\varepsilon_{\text {agg. }}$. While the approximation of the $\rho_{e}$ employed in this model lacks the experimental accuracy achieved for $\rho_{e}$ measurements afforded by VCM, the ISDD model does allow for time-resolved approximation of NP transport parameters within unstable dispersions. Employing the ISDD model, $\alpha$ values were obtained using time-resolved $d_{h}$ values and the dosimetry curves for both the nZnO/PBS and $\mathrm{nZnO}$ - FBS/PBS stock dispersions in RPMI-based cellular media were modeled for several time points from 0 to 24 hours. These time-resolved points were compiled to generate ISDD curves which were then fit to both the $f_{D}(t)$ and $f_{A}(t)$ mathematic models to obtain $\alpha$ values for each representative graph (Figure 2.13C and D). For the unstable $\mathrm{nZnO} / \mathrm{PBS}$ stock, the ISDD $\alpha$ values of 0.290 for adherent cell model conditions and 0.120 for the suspension cell models were significantly higher (1037\% and $1279 \%$ increases for adherent and suspension cell models, respectively) than those obtained using the Harvard VCM (Table 2.1). The ISDD modeled $t_{90}$ values of 7.94 (adherent cells) and 19.12 (suspension cells), were more consistent with the experimental stability data discussed earlier. Applying the same model to the stable nZnO - FBS/PBS stock dispersion yielded $\alpha$ values ( 0.331 and 0.107 for adherent and suspension cell models, respectively) more closely aligned to the VCM determined values (Table 2.1) with percent increases of $107 \%$ and $97 \%$ for adherent and suspension cell models, respectively. Surprisingly, the ISDD 
modeled curves for the nZnO/PBS (unstable) and $\mathrm{nZnO}$ - FBS/PBS (stable) dispersions imply similar dosimetry kinetics for both cellular models and, if considered without experimentally derived stability profiles (e.g. average hydrodynamic size profiles, size distribution histograms and sedimentation analysis), would not reflect the true dosimetry for unstable stock dispersions. While the ISDD derived $f_{D}(t)$ curve for the unstable nZnO/PBS stock dispersion (Figure 2.13C) at face value implies stable dosimetry kinetics, the individual ISDD curves at each time point reflect the unstable nature of the $\mathrm{nZnO} / \mathrm{PBS}$ dispersion (Figure 2.14A). As a result, using either mathematical model to predict the effective dose for this dispersion would not be advisable. For the stable nZnO - FBS/PBS dispersion, both the VCM and ISDD models (Figure 2.13 and Figure 2.14B) depict stable dosimetry kinetics for both suspension and adherent cell models. For adherent cells, the t90 value of 14.5 hours (Table 2.1; VCM) suggests $90 \%$ of the NPs in suspension will come in contact with and potentially interact with the cell layer by the 24-hour time point indicating that the experimentally observed toxicity values are an accurate assessment of the in vitro toxicity for adherent cell types. Mathematical models for stable dispersions in suspension cells may provide less definitive information regarding effective dose. While these models can accurately predict the number of NPs remaining in dispersion as a function of time $\left[f_{A}(t)\right]$, the models fail to account for NP-cellular interactions that will occur within the suspension culture before sedimentation processes remove NPs from the dispersion. Better mathematical models are needed to predict the fate and transport of NPs in suspension cell models that include NP sedimentation rates as well as time-resolved NPcellular interactions. Taken together, these observations underscore the importance of time- 
dependent stability studies for NP stock dispersions prior to the implementation of fate and transport dosimetry models and downstream experimental applications.

\section{Conclusions}

Studies reported in this work show that $\mathrm{ZnO}$ nanoparticles form very large agglomerates with hydrodynamic sizes in the 300 to $20,000 \mathrm{~nm}$ range which depend primarily on the solution composition used. Presence of such agglomerates leads to sedimentation of a significant portion of the nanoparticles which increases in time. For adherent cells, this will increase the effective NP concentration interacting with the cells at very early time points and may cause unusually large and potentially inaccurate in vitro toxicity measurements. This was demonstrated using two adherent epithelial cell lines (T-47D and LNCaP) with both cell types experiencing significant decreases in NPmediated cell death with the more stable NP stock dispersions. Likewise, this sedimentation process will reduce the effective NP concentration remaining in the solution medium. Subsequent investigations using suspension cells showed that this phenomenon results in an opposite trend due to the reduction in the number of nanocrystals interacting with the suspension cells and consequently, a lower than actual toxic response is obtained. Using a $10 \%$ FBS/PBS-stock to disperse the same $\mathrm{nZnO}$, the $\mathrm{IC}_{50}$ values for Jurkat (Hut) cancer cells decreased from $0.39 \mathrm{mM}(0.21 \mathrm{mM})$ for $\mathrm{nZnO} / \mathrm{PBS}$ treated cells to $0.27 \mathrm{mM}$ $(0.14 \mathrm{mM})$ for the $\mathrm{nZnO}-\mathrm{FBS} / \mathrm{PBS}$ dispersion. Their normal counterparts $\left(\mathrm{CD} 4^{+} \mathrm{T}^{-}\right.$ lymphocytes) also showed a reduction in $\mathrm{IC}_{50}$ with $10 \% \mathrm{FBS}$ addition. $\mathrm{Its} \mathrm{IC}_{50}$ decreased from $4.69 \mathrm{mM}$ for $\mathrm{nZnO} / \mathrm{PBS}$ dispersions to $2.43 \mathrm{mM}$ when $\mathrm{nZnO}-\mathrm{FBS} / \mathrm{PBS}$ stock was used. On the other hand, treating the adherent cells T-47D and LNCaP using the $\mathrm{nZnO}-$ FBS/PBS dispersion resulted in $48.7 \%$ and $71.4 \%$ reductions, respectively, in the NP- 
induced toxic response compared to $\mathrm{nZnO} / \mathrm{PBS}$ dispersion. Surface modification of these $\mathrm{nZnO}$ using serum proteins seems to be a suitable approach to obtain stable dispersions and accurate cytotoxicity values, thus improving their utility in therapeutic applications.

This study has shown the important role of serum proteins in modifying the $\mathrm{ZnO}$ nanocrystal surface resulting in the formation of considerably smaller agglomerates and stable NP dispersions. Furthermore, these FBS surface coatings influenced $\mathrm{Zn}^{2+}$ release from the nanocrystals and induced changes in the mitochondrial function of the cells as evidenced by the increased ROS production in the $\mathrm{nZnO}-$ FBS/PBS treated cells. Given these findings, it is readily apparent that the addition of serum proteins adds a level of complexity to our experimental understanding of NP-cell interactions. Further studies are needed to examine how FBS-coatings change NP-cellular surface interactions, influence cellular uptake mechanisms and modify molecular interactions with other extracellular matrix components. Additionally, these studies should be extended to include newer 3D cellular culture models which often employ complex biomolecular solution systems or molecular scaffolds to better mimic in vivo-like environments for mechanistic biological assays $[71,72]$. These complex culture systems will influence the formation of the NP biomolecular corona and change the diffusion and sedimentation rates for NP dispersions especially those containing additional serum proteins. Furthermore, 3D culture systems alter cellular growth patterns, metabolism, genetic expression and phenotype organization changing NP-induced cellular responses which could be further influenced by the addition of NP-coating materials $[72,73]$. Finally, these studies should be extended into other cell types. Molecular coatings are often considered as a means to achieve safer $\mathrm{nZnO}$ structures, 
yet little is known about how these molecular coatings will influence NP-cellular interactions with endothelial cells $[18,27,74]$ and other primary immune system cells [10].

\section{Competing Interests}

The authors declare that they have no competing interests.

\section{Authors' Contributions}

CBA designed the experiment, synthesized and characterized the NP samples, performed the stability measurements and biological assays. JJC assisted in the nanoparticle synthesis, dispersion stability assays and performed the TEM measurements. CBA and AP wrote the manuscript. AP and DGW participated in the experimental design process and coordination of the study. All authors read and approved the final manuscript.

\section{Acknowledgements}

This work was supported in part by NSF CBET 1134468, NSF EAGER DMR1137419, ARO W911NF-09-1-0051, and NIH 1R15CA141358 grants. The authors thank Dr. Marion Lytle and the Biotrace Lab at Boise State University for the ICP-MS use. Additionally, we thank Prof. Ben McMorran and CAMCOR for TEM images, CAMCOR's Titan and Tecnai are supported by W.M Keck Foundation, ONAMI, the Air Force Research Laboratory (agreement number FA8650-05-1-5041), and University of Oregon Office of Research. 


\section{References}

1] Guo, W. S.; Yang, W. T.; Wang, Y.; Sun, X. L.; Liu, Z. Y.; Zhang, B. B.; Chang, J.; Chen, X. Y. Color-tunable gd-zn-cu-in-s/zns quantum dots for dual modality magnetic resonance and fluorescence imaging. Nano Research 2014, 7, 1581-1591.

2] Pang, Y. F.; Rong, Z.; Wang, J. F.; Xiao, R.; Wang, S. Q. A fluorescent aptasensor for h5n1 influenza virus detection based-on the core-shell nanoparticles metalenhanced fluorescence (mef). Biosensors \& Bioelectronics 2015, 66, 527-532.

3] Andreu, I.; Natividad, E.; Solozabal, L.; Roubeau, O. Nano-objects for addressing the control of nanoparticle arrangement and performance in magnetic hyperthermia. ACS Nano 2015, 9, 1408-1419.

4] Nazli, C.; Demirer, G. S.; Yar, Y.; Acar, H. Y.; Kizilel, S. Targeted delivery of doxorubicin into tumor cells via mmp-sensitive peg hydrogel-coated magnetic iron oxide nanoparticles (mionps). Colloids and Surfaces B-Biointerfaces 2014, 122, 674-683.

5] Luo, C.; Sun, J.; Sun, B. J.; He, Z. G. Prodrug-based nanoparticulate drug delivery strategies for cancer therapy. Trends in Pharmacological Sciences 2014, 35, 12-22.

6] Huang, X.; Wang, Z.; Dai, H.; Wang, C. M.; Xia, B.; Chen, L.; Pan, J. Docetaxel grafted magnetic nanoparticles as dual-therapeutic agentia for targeting perfusion therapy of urethral carcinoma. Journal of Nanoparticle Research 2014, 16, 1-11.

7] Zhang, H. Y.; Ji, Z. X.; Xia, T.; Meng, H.; Low-Kam, C.; Liu, R.; Pokhrel, S.; Lin, S. J.; Wang, X.; Liao, Y. P. et al. Use of metal oxide nanoparticle band gap to develop a predictive paradigm for oxidative stress and acute pulmonary inflammation. ACS Nano 2012, 6, 4349-4368.

8] Horie, M.; Kato, H.; Fujita, K.; Endoh, S.; Iwahashi, H. In vitro evaluation of cellular response induced by manufactured nanoparticles. Chemical Research in Toxicology 2012, 25, 605-619.

9] Cronholm, P.; Karlsson, H. L.; Hedberg, J.; Lowe, T. A.; Winnberg, L.; Elihn, K.; Wallinder, I. O.; Moller, L. Intracellular uptake and toxicity of ag and cuo nanoparticles: A comparison between nanoparticles and their corresponding metal ions. Small 2013, 9, 970-982. 
10] Hanley, C.; Thurber, A.; Hanna, C.; Punnoose, A.; Zhang, J. H.; Wingett, D. G. The influences of cell type and zno nanoparticle size on immune cell cytotoxicity and cytokine induction. Nanoscale Research Letters 2009, 4, 1409-1420.

11] Xu, M.; Fujita, D.; Kajiwara, S.; Minowa, T.; Li, X.; Takemura, T.; Iwai, H.; Hanagata, N. Contribution of physicochemical characteristics of nano-oxides to cytotoxicity. Biomaterials 2010, 31, 8022-8031.

12] Heng, B. C.; Zhao, X.; Tan, E. C.; Khamis, N.; Assodani, A.; Xiong, S.; Ruedl, C.; $\mathrm{Ng}, \mathrm{K}$. W.; Loo, J. S. Evaluation of the cytotoxic and inflammatory potential of differentially shaped zinc oxide nanoparticles. Arch Toxicol 2011, 85, 1517-28.

13] Zhang, J. H.; Dong, G. J.; Thurber, A.; Hou, Y. Y.; Gu, M.; Tenne, D. A.; Hanna, C. B.; Punnoose, A. Tuning the properties of zno, hematite, and ag nanoparticles by adjusting the surface charge. Advanced Materials 2012, 24, 1232-1237.

14] Hsiao, I. L.; Huang, Y. J. Effects of various physicochemical characteristics on the toxicities of zno and tio2 nanoparticles toward human lung epithelial cells. Sci. Total Environ. 2011, 409, 1219-1228.

15] Reddy, K. M.; Feris, K.; Bell, J.; Wingett, D. G.; Hanley, C.; Punnoose, A. Selective toxicity of zinc oxide nanoparticles to prokaryotic and eukaryotic systems. Applied Physics Letters 2007, 90, 1-8.

16] Hanley, C.; Layne, J.; Punnoose, A.; Reddy, K. M.; Coombs, I.; Coombs, A.; Feris, K.; Wingett, D. Preferential killing of cancer cells and activated human $\mathrm{t}$ cells using zno nanoparticles. Nanotechnology 2008, 19, 1-10.

17] Feris, K.; Otto, C.; Tinker, J.; Wingett, D.; Punnoose, A.; Thurber, A.; Kongara, M.; Sabetian, M.; Quinn, B.; Hanna, C. et al. Electrostatic interactions affect nanoparticle-mediated toxicity to gram-negative bacterium pseudomonas aeruginosa pao1. Langmuir 2010, 26, 4429-4436.

18] Setyawati, M. I.; Tay, C. Y.; Chia, S. L.; Goh, S. L.; Fang, W.; Neo, M. J.; Chong, H. C.; Tan, S. M.; Loo, S. C. J.; Ng, K. W. et al. Titanium dioxide nanomaterials cause endothelial cell leakiness by disrupting the homophilic interaction of vecadherin. Nature Communications 2013, 4, 1-12.

19] Sasidharan, A.; Chandran, P.; Menon, D.; Raman, S.; Nair, S.; Koyakutty, M. Rapid dissolution of zno nanocrystals in acidic cancer microenvironment leading to preferential apoptosis. Nanoscale Research Letters 2011, 3, 3657-3669. 
20] Akhtar, M. J.; Ahamed, M.; Kumar, S.; Khan, M. M.; Ahmad, J.; Alrokayan, S. A. Zinc oxide nanoparticles selectively induce apoptosis in human cancer cells through reactive oxygen species. Int J Nanomedicine 2012, 7, 845-857.

21] Wong, A. D.; Ye, M.; Ulmschneider, M. B.; Searson, P. C. Quantitative analysis of the enhanced permeation and retention (epr) effect. PloS one 2015, 10, 1-13.

22] Liu, H. H.; Surawanvijit, S.; Rallo, R.; Orkoulas, G.; Cohen, Y. Analysis of nanoparticle agglomeration in aqueous suspensions via constant-number monte carlo simulation. Environmental Science \& Technology 2011, 45, 9284-9292.

23] Keller, A. A.; Wang, H.; Zhou, D.; Lenihan, H. S.; Cherr, G.; Cardinale, B. J.; Miller, R.; Ji, Z. Stability and aggregation of metal oxide nanoparticles in natural aqueous matrices. Environmental Science \& Technology 2010, 44, 1962-1967.

24] Jassby, D.; Farner Budarz, J.; Wiesner, M. Impact of aggregate size and structure on the photocatalytic properties of tio2 and zno nanoparticles. Environ Sci Technol 2012, 46, 6934-41.

25] Horie, M.; Nishio, K.; Fujita, K.; Kato, H.; Endoh, S.; Suzuki, M.; Nakamura, A.; Miyauchi, A.; Kinugasa, S.; Yamamoto, K. et al. Cellular responses by stable and uniform ultrafine titanium dioxide particles in culture-medium dispersions when secondary particle size was $100 \mathrm{~nm}$ or less. Toxicol. Vitro 2010, 24, 1629-1638.

26] Maiorano, G.; Sabella, S.; Sorce, B.; Brunetti, V.; Malvindi, M. A.; Cingolani, R.; Pompa, P. P. Effects of cell culture media on the dynamic formation of proteinnanoparticle complexes and influence on the cellular response. Acs Nano 2010, 4, 7481-7491.

27] Setyawati, M. I.; Tay, C. Y.; Docter, D.; Stauber, R. H.; Leong, D. T. Understanding and exploiting nanoparticles' intimacy with the blood vessel and blood. Chemical Society Reviews 2015, 8174-8199.

28] Brayner, R.; Dahoumane, S. A.; Yepremian, C.; Djediat, C.; Meyer, M.; Coute, A.; Fievet, F. Zno nanoparticles: Synthesis, characterization, and ecotoxicological studies. Langmuir 2010, 26, 6522-6528.

29] Sabuncu, A. C.; Grubbs, J.; Qian, S. Z.; Abdel-Fattah, T. M.; Stacey, M. W.; Beskok, A. Probing nanoparticle interactions in cell culture media. Colloids and Surfaces B-Biointerfaces 2012, 95, 96-102.

30] Wells, M. A.; Abid, A.; Kennedy, I. M.; Barakat, A. I. Serum proteins prevent aggregation of fe2o3 and zno nanoparticles. Nanotoxicology 2012, 6, 837-846. 
31] Xu, M.; Li, J.; Iwai, H.; Mei, Q.; Fujita, D.; Su, H.; Chen, H.; Hanagata, N. Formation of nano-bio-complex as nanomaterials dispersed in a biological solution for understanding nanobiological interactions. Sci Rep 2012, 2, 1-6.

32] DeLoid, G.; Cohen, J. M.; Darrah, T.; Derk, R.; Rojanasakul, L.; Pyrgiotakis, G.; Wohlleben, W.; Demokritou, P. Estimating the effective density of engineered nanomaterials for in vitro dosimetry. Nature Communications 2014, 5.

33] Cohen, J. M.; Teeguarden, J. G.; Demokritou, P. An integrated approach for the in vitro dosimetry of engineered nanomaterials. Part. Fibre Toxicol. 2014, 11.

34] Cho, E. C.; Zhang, Q.; Xia, Y. The effect of sedimentation and diffusion on cellular uptake of gold nanoparticles. Nature Nanotechnology 2011, 6, 385-391.

35] Xia, X. R.; Monteiro-Riviere, N. A.; Riviere, J. E. An index for characterization of nanomaterials in biological systems. Nature Nanotechnology 2010, 5, 671-675.

36] Monopoli, M. P.; Aberg, C.; Salvati, A.; Dawson, K. A. Biomolecular coronas provide the biological identity of nanosized materials. Nature Nanotechnology 2012, 7, 779-786.

37] Tenzer, S.; Docter, D.; Kuharev, J.; Musyanovych, A.; Fetz, V.; Hecht, R.; Schlenk, F.; Fischer, D.; Kiouptsi, K.; Reinhardt, C. et al. Rapid formation of plasma protein corona critically affects nanoparticle pathophysiology. Nature Nanotechnology 2013, 8, 772-781.

38] Lesniak, A.; Fenaroli, F.; Monopoli, M. R.; Aberg, C.; Dawson, K. A.; Salvati, A. Effects of the presence or absence of a protein corona on silica nanoparticle uptake and impact on cells. Acs Nano 2012, 6, 5845-5857.

39] Ge, C. C.; Du, J. F.; Zhao, L. N.; Wang, L. M.; Liu, Y.; Li, D. H.; Yang, Y. L.; Zhou, R. H.; Zhao, Y. L.; Chai, Z. F. et al. Binding of blood proteins to carbon nanotubes reduces cytotoxicity. Proceedings of the National Academy of Sciences of the United States of America 2011, 108, 16968-16973.

40] Hu, W. B.; Peng, C.; Lv, M.; Li, X. M.; Zhang, Y. J.; Chen, N.; Fan, C. H.; Huang, $\mathrm{Q}$. Protein corona-mediated mitigation of cytotoxicity of graphene oxide. Acs Nano 2011, 5, 3693-3700.

41] Hsiao, I. L.; Huang, Y. J. Effects of serum on cytotoxicity of nano- and micro-sized zno particles. Journal of Nanoparticle Research 2013, 15, 1-16. 
42] Yin, H.; Casey, P. S.; McCall, M. J.; Fenech, M. Effects of surface chemistry on cytotoxicity, genotoxicity, and the generation of reactive oxygen species induced by zno nanoparticles. Langmuir 2010, 26, 15399-408.

43] Hinderliter, P. M.; Minard, K. R.; Orr, G.; Chrisler, W. B.; Thrall, B. D.; Pounds, J. G.; Teeguarden, J. G. Isdd: A computational model of particle sedimentation, diffusion and target cell dosimetry for in vitro toxicity studies. Part. Fibre Toxicol. 2010, 7.

44] Sterling, M. C.; Bonner, J. S.; Ernest, A. N. S.; Page, C. A.; Autenrieth, R. L. Application of fractal flocculation and vertical transport model to aquatic solsediment systems. Water Research 2005, 39, 1818-1830.

45] LUTTEROTTI, L.; SCARDI, P.; MAISTRELLI, P. Ls1 - a computer-program for simultaneous refinement of material structure and microstructure. Journal of Applied Crystallography 1992, 25, 459-462.

46] Taurozzi, J. S.; Hackley, V. A.; Wiesner, M. R. Ultrasonic dispersion of nanoparticles for environmental, health and safety assessment - issues and recommendations. Nanotoxicology 2011, 5, 711-729.

47] Cohen, J.; DeLoid, G.; Pyrgiotakis, G.; Demokritou, P. Interactions of engineered nanomaterials in physiological media and implications for in vitro dosimetry. Nanotoxicology 2013, 7, 417-431.

48] Mukhopadhyay, P.; Rajesh, M.; Haskó, G.; Hawkins, B. J.; Madesh, M.; Pacher, P. Simultaneous detection of apoptosis and mitochondrial superoxide production in live cells by flow cytometry and confocal microscopy. Nature protocols $\mathbf{2 0 0 7 , 2 ,}$ 2295-2301.

49] Xie, Y. X.; Lutterotti, L.; Wenk, H. R.; Kovacs, F. Texture analysis of ancient coins with tof neutron diffraction. Journal of Materials Science 2004, 39, 3329-3337.

50] Fukuzaki, S.; Urano, H.; Nagata, K. Adsorption of bovine serum albumin onto metal oxide surfaces. Journal of Fermentation and Bioengineering 1996, 81, 163167.

51] Joshi, S.; Ghosh, I.; Pokhrel, S.; Madler, L.; Nau, W. M. Interactions of amino acids and polypeptides with metal oxide nanoparticles probed by fluorescent indicator adsorption and displacement. Acs Nano 2012, 6, 5668-5679. 
52] Farbun, I. A.; Romanova, I. V.; Terikovskaya, T. E.; Dzanashvili, D. I.; Kirillov, S. A. Complex formation in the course of synthesis of zinc oxide from citrate solutions. Russian Journal of Applied Chemistry 2007, 80, 1798-1803.

53] Max, J. J.; Chapados, C. Infrared spectroscopy of aqueous carboxylic acids: Comparison between different acids and their salts. Journal of Physical Chemistry A 2004, 108, 3324-3337.

54] Max, J. J.; Chapados, C. Infrared spectroscopy of aqueous carboxylic acids: Malic acid. Journal of Physical Chemistry A 2002, 106, 6452-6461.

55] Roach, P.; Farrar, D.; Perry, C. C. Interpretation of protein adsorption: Surfaceinduced conformational changes. Journal of the American Chemical Society 2005, $127,8168-8173$.

56] Saha, B.; Das, G. Malachite nanoparticle: A new basic hydrophilic surface for phcontrolled adsorption of bovine serum albumin with a high loading capacity. $J$. Phys. Chem. C 2009, 113, 15667-15675.

57] Chittur, K. K. Ftir/atr for protein adsorption to biomaterial surfaces. Biomaterials 1998, 19, 357-369.

58] Bian, S.-W.; Mudunkotuwa, I. A.; Rupasinghe, T.; Grassian, V. H. Aggregation and dissolution of $4 \mathrm{~nm}$ zno nanoparticles in aqueous environments: Influence of ph, ionic strength, size, and adsorption of humic acid. Langmuir 2011, 27, 60596068.

59] Horie, M.; Nishio, K.; Fujita, K.; Endoh, S.; Miyauchi, A.; Saito, Y.; Iwahashi, H.; Yamamoto, K.; Murayama, H.; Nakano, H. et al. Protein adsorption of ultrafine metal oxide and its influence on cytotoxicity toward cultured cells. Chemical Research in Toxicology 2009, 22, 543-553.

60] Chakraborti, S.; Joshi, P.; Chakravarty, D.; Shanker, V.; Ansari, Z. A.; Singh, S. P.; Chakrabarti, P. Interaction of polyethyleneimine-functionalized zno nanoparticles with bovine serum albumin. Langmuir 2012, 28, 11142-52.

61] Lv, J. T.; Zhang, S. Z.; Luo, L.; Han, W.; Zhang, J.; Yang, K.; Christie, P. Dissolution and microstructural transformation of zno nanoparticles under the influence of phosphate. Environmental Science \& Technology 2012, 46, 72157221.

62] Tantra, R.; Jing, S. H.; Pichaimuthu, S. K.; Walker, N.; Noble, J.; Hackley, V. A. Dispersion stability of nanoparticles in ecotoxicological investigations: The need 
for adequate measurement tools. Journal of Nanoparticle Research 2011, 13, 37653780 .

63] Tripathy, N.; Hong, T. K.; Ha, K. T.; Jeong, H. S.; Hahn, Y. B. Effect of zno nanoparticles aggregation on the toxicity in raw 264.7 murine macrophage. Journal of hazardous materials 2014, 270, 110-117.

64] Mu, Q.; David, C. A.; Galceran, J.; Rey-Castro, C.; Krzeminski, L.; Wallace, R.; Bamiduro, F.; Milne, S. J.; Hondow, N. S.; Brydson, R. et al. Systematic investigation of the physicochemical factors that contribute to the toxicity of zno nanoparticles. Chemical Research in Toxicology 2014, 27, 558-567.

65] Reed, R. B.; Ladner, D. A.; Higgins, C. P.; Westerhoff, P.; Ranville, J. F. Solubility of nano-zinc oxide in environmentally and biologically important matrices. Environmental Toxicology and Chemistry 2012, 31, 93-99.

66] Ramasamy, M.; Das, M.; An, S. S. A.; Yi, D. K. Role of surface modification in zinc oxide nanoparticles and its toxicity assessment toward human dermal fibroblast cells. International journal of nanomedicine 2014, 9, 3707-3718.

67] Shen, C. C.; James, S. A.; de Jonge, M. D.; Turney, T. W.; Wright, P. F. A.; Feltis, B. N. Relating cytotoxicity, zinc ions, and reactive oxygen in zno nanoparticleexposed human immune cells. Toxicological Sciences 2013, 136, 120130.

68] Yu, K. N.; Yoon, T. J.; Minai-Tehrani, A.; Kim, J. E.; Park, S. J.; Jeong, M. S.; Ha, S. W.; Lee, J. K.; Kim, J. S.; Cho, M. H. Zinc oxide nanoparticle induced autophagic cell death and mitochondrial damage via reactive oxygen species generation. Toxicology in Vitro 2013, 27, 1187-1195.

69] Osmond-McLeod, M. J.; Osmond, R. I. W.; Oytam, Y.; McCall, M. J.; Feltis, B.; Mackay-Sim, A.; Wood, S. A.; Cook, A. L. Surface coatings of zno nanoparticles mitigate differentially a host of transcriptional, protein and signalling responses in primary human olfactory cells. Part. Fibre Toxicol. 2013, 10.

70] Everett, W. N.; Chern, C.; Sun, D.; McMahon, R. E.; Zhang, X.; Chen, W.-J. A.; Hahn, M. S.; Sue, H. J. Phosphate-enhanced cytotoxicity of zinc oxide nanoparticles and agglomerates. Toxicology Letters 2014, 225, 177-184.

71] Hutmacher, D. W. Biomaterials offer cancer research the third dimension. Nature Materials 2010, 9, 90-93. 
72] Leong, D. T.; Ng, K. W. Probing the relevance of $3 \mathrm{~d}$ cancer models in nanomedicine research. Adv. Drug Deliv. Rev. 2014, 79-80, 95-106.

73] Chia, S. L.; Tay, C. Y.; Setyawati, M. I.; Leong, D. T. Biomimicry 3d gastrointestinal spheroid platform for the assessment of toxicity and inflammatory effects of zinc oxide nanoparticles. Small 2015, 11, 702-712.

74] Tay, C. Y.; Setyawati, M. I.; Xie, J.; Parak, W. J.; Leong, D. T. Back to basics: Exploiting the innate physico-chemical characteristics of nanomaterials for biomedical applications. Advanced Functional Materials 2014, 24, 5936-5955. 
Tables and Figures

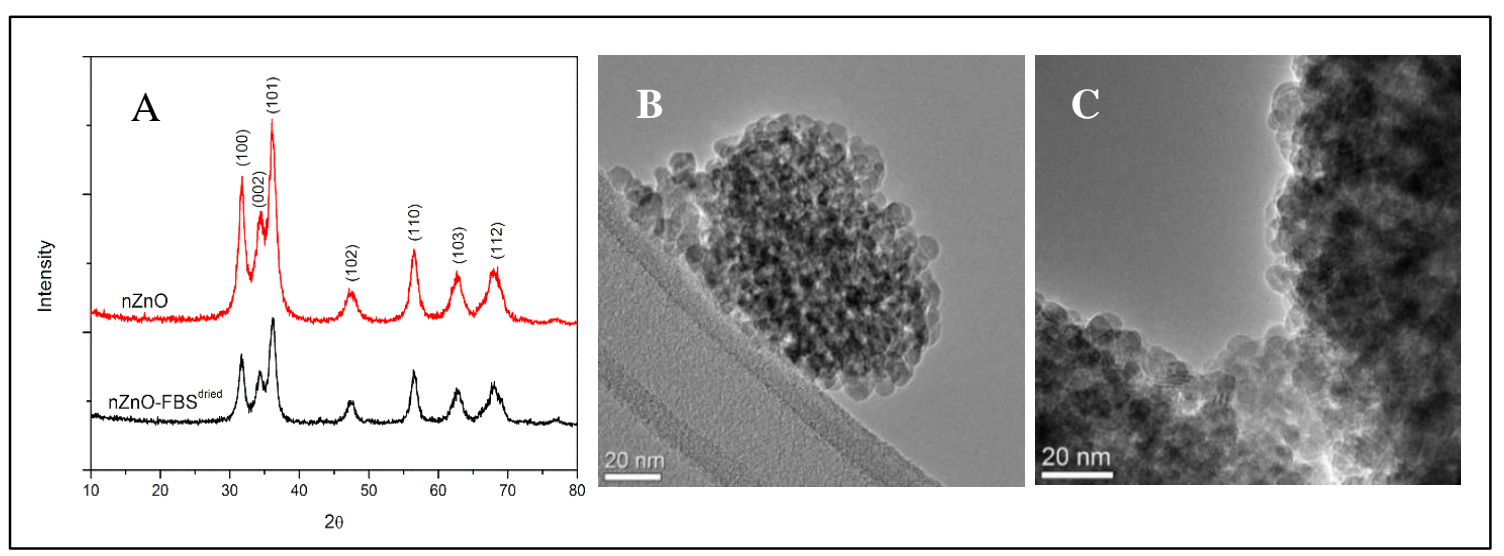

Figure 2.1 Crystal phase composition, size and morphology characterization for nZnO and nZnO - FBS ${ }^{\text {dried }}$ NPs. A) XRD spectra and TEM images for B) $n Z n O$ and C) $\mathbf{n Z n O}-$ FBS $^{\text {dried }}$. 


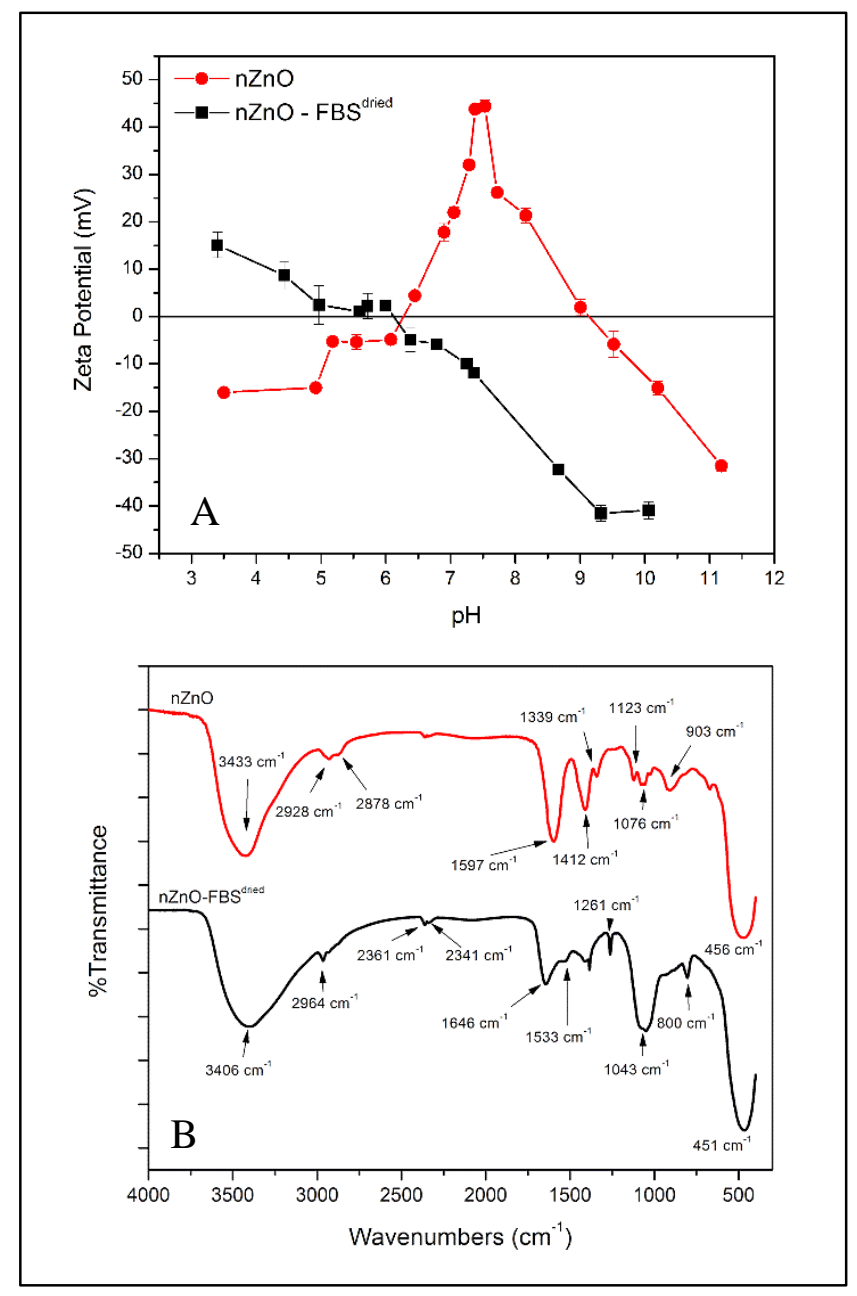

Figure 2.2 Surface property analysis for $\mathrm{nZnO}$ and $\mathrm{nZnO}-\mathrm{FBS}^{\text {dried }}$. A) Zeta potentials measured as a function of $\mathrm{pH}$ and B) FTIR spectra with the wavenumbers of the main peaks marked. 


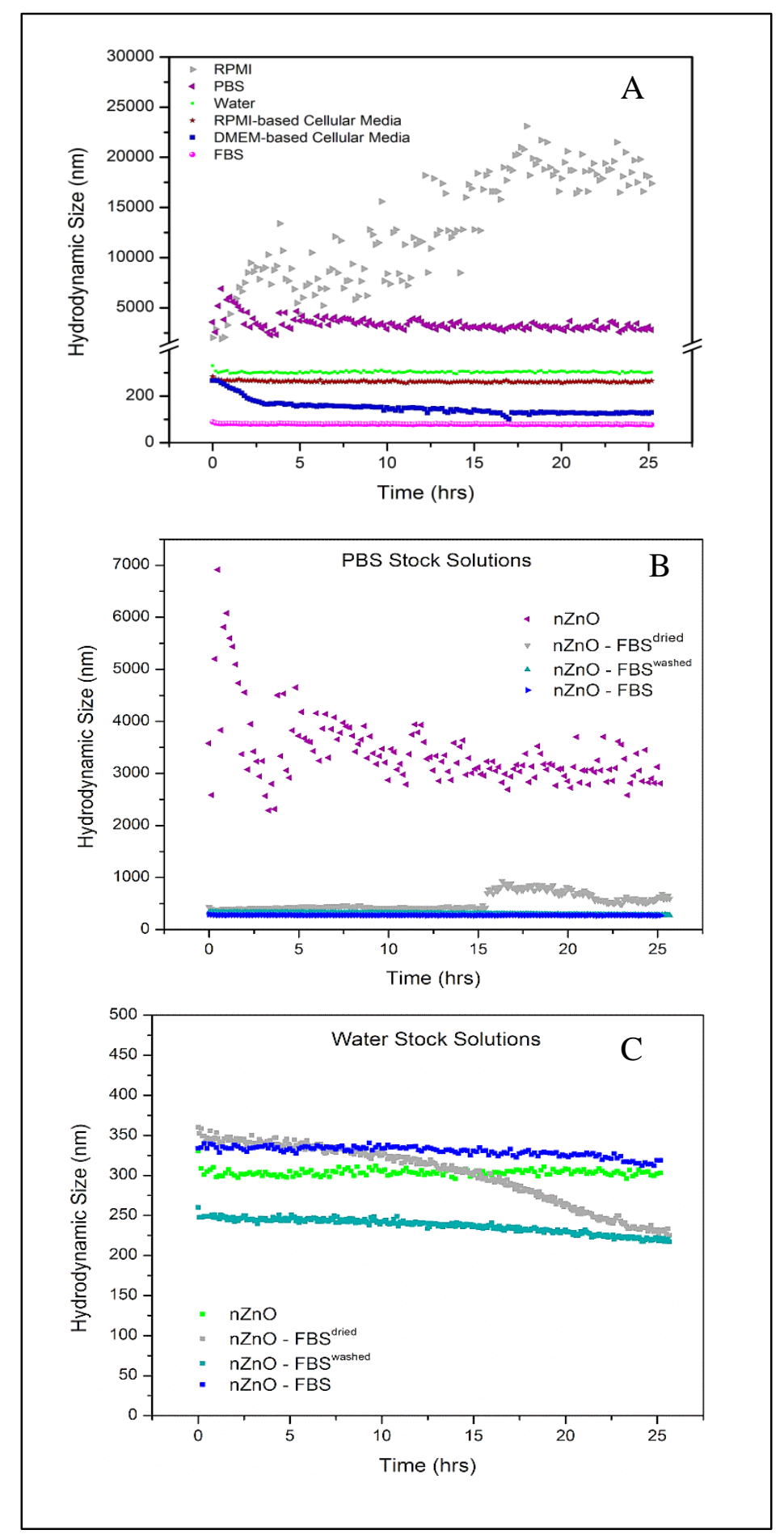

Figure 2.3 Aggregation profile evaluation for NP stock solutions. Average hydrodynamic size profiles of $25 \mathrm{mM} \mathrm{ZnO}$ stock dispersions $(\mathrm{pH}=7.3)$ for a 24-hour time period. A) $\mathrm{nZnO}$ in various biologically relevant solutions. $\mathrm{B}$ ) $\mathrm{nZnO}, \mathrm{nZnO}-$ FBS $^{\text {dried }}$, nZnO - FBS ${ }^{\text {washed }}$ and $\mathbf{n Z n O}$ - FBS stock dispersions in PBS and C) $\mathbf{n Z n O}$, nZnO - FBS ${ }^{\text {dried }}$, nZnO - FBS $^{\text {washed }}$ and nZnO - FBS stock dispersions in nanopure water. 

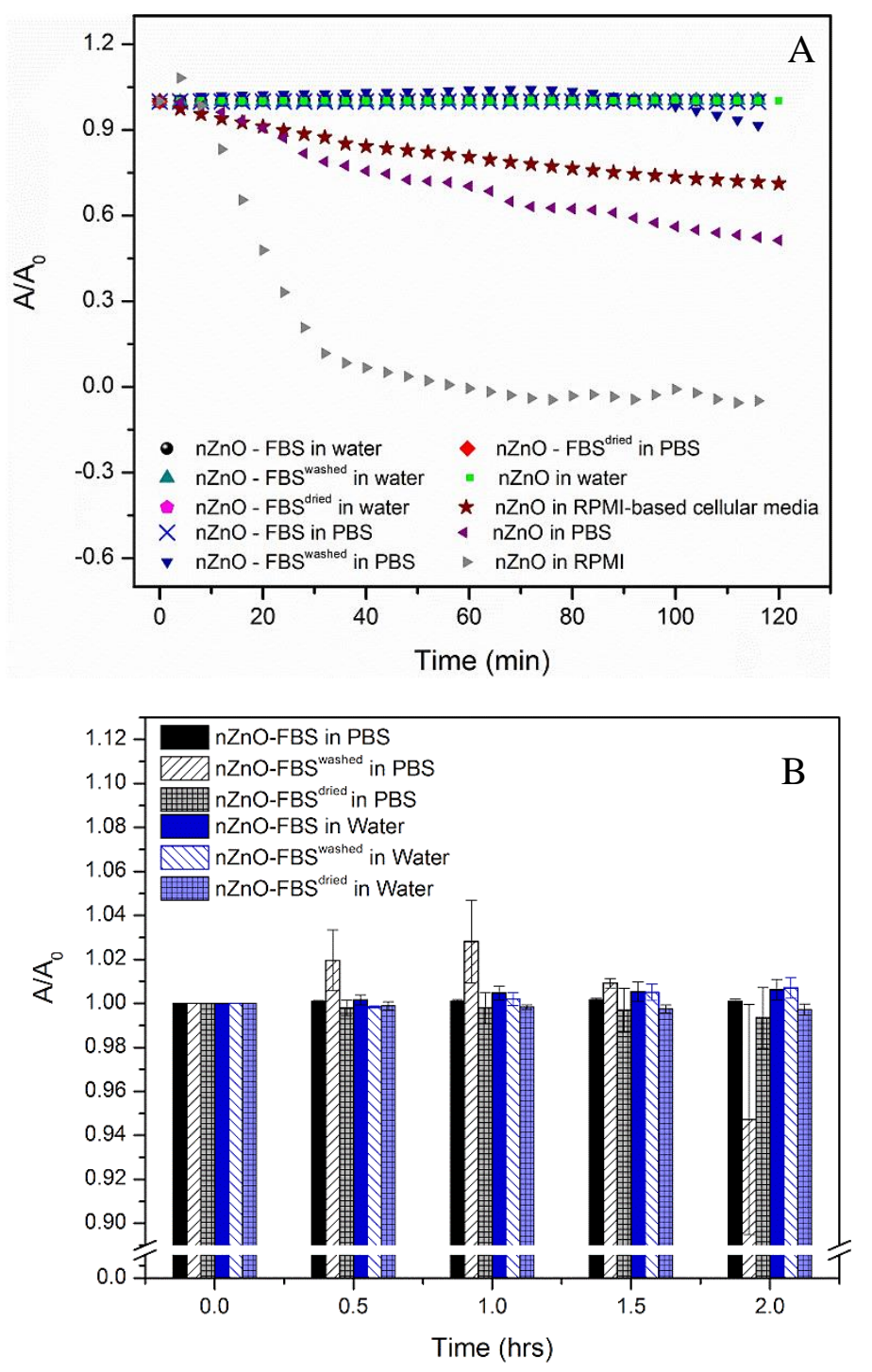

Figure 2.4 Sedimentation profile evaluation for NP stock dispersions. Sedimentation profiles of $25 \mathrm{mM} \mathrm{ZnO}$ stock dispersions $(\mathrm{pH}=7.3$ ) for a 2-hour time period. A) nZnO, nZnO - FBS ${ }^{\text {dried, }}$ nZnO - FBS ${ }^{\text {washed }}$ and $\mathbf{n Z n O}$ - FBS stock dispersions in various biologically relevant solutions. B) Sedimentation histograms for $0,0.5,1.0,1.5$ and 2.0 hour time points for $\mathrm{nZnO}-\mathrm{FBS}^{\text {dried }}, \mathrm{nZnO}-\mathrm{FBS}^{\text {washed }}$ and nZnO - FBS stock dispersions in PBS and nanopure water. 


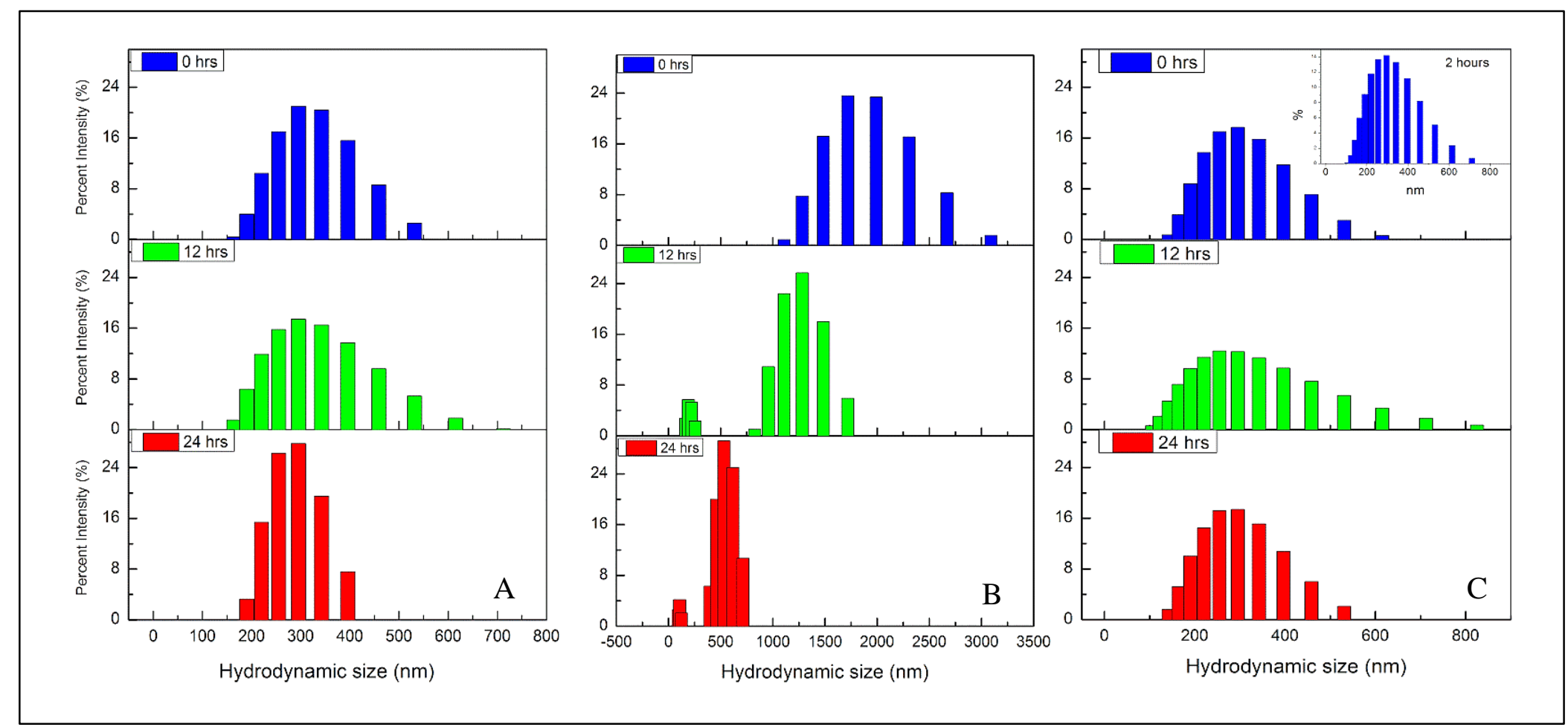

Figure 2.5 Hydrodynamic size distribution profiles at 0, 12 and 24 hours. Histograms represent $25 \mathrm{mM}$ stock dispersions prepared in A) nanopure water B) PBS and C) RPMI-based cellular media. 


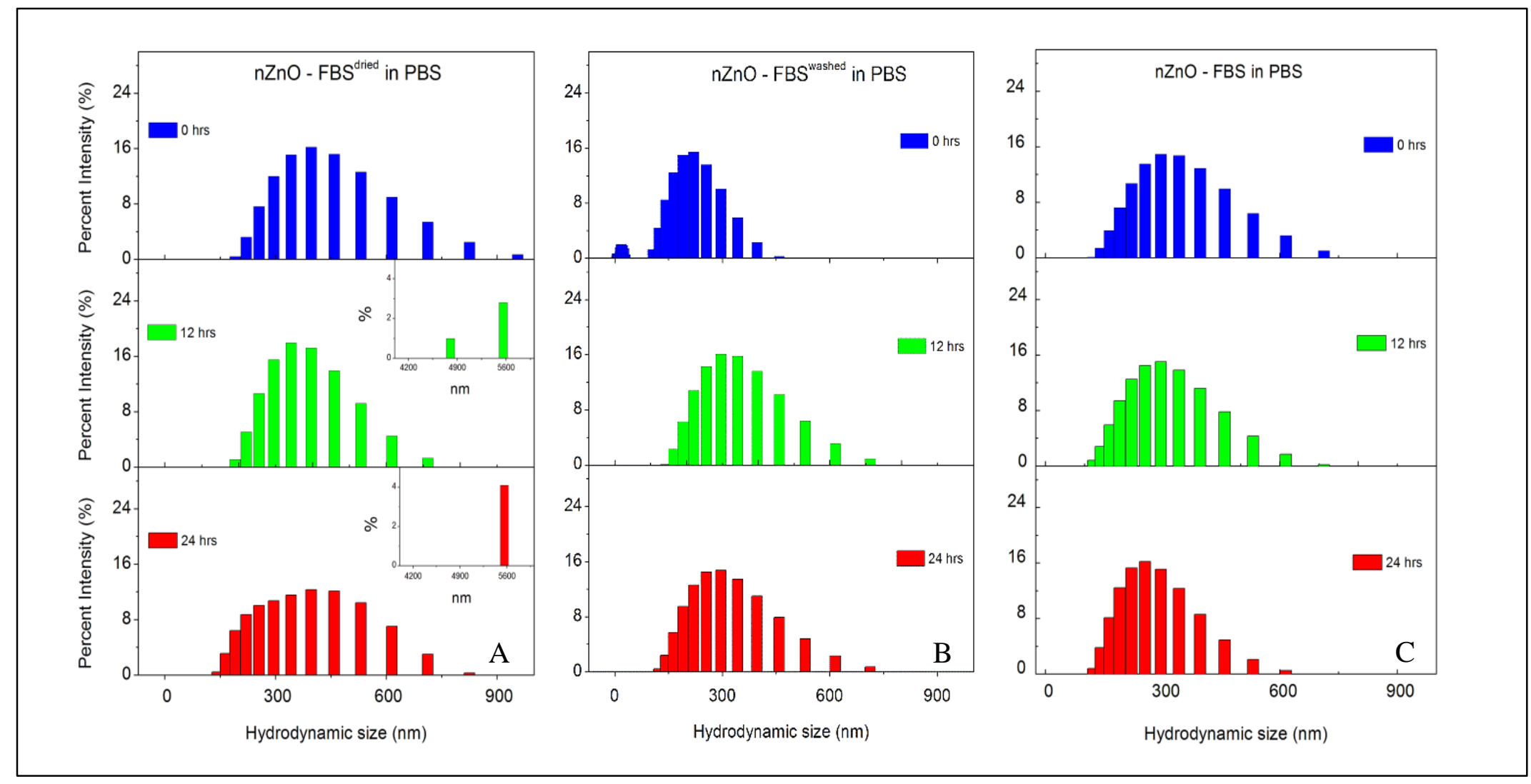

Figure 2.6 Hydrodynamic size distribution profiles at 0,12 and 24 hours. Histograms represent $25 \mathrm{mM}$ stock dispersions prepared in PBS for A) nZnO - FBS ${ }^{\text {dried }}$, B) nZnO - FBS ${ }^{\text {washed }}$ and C) nZnO - FBS. 


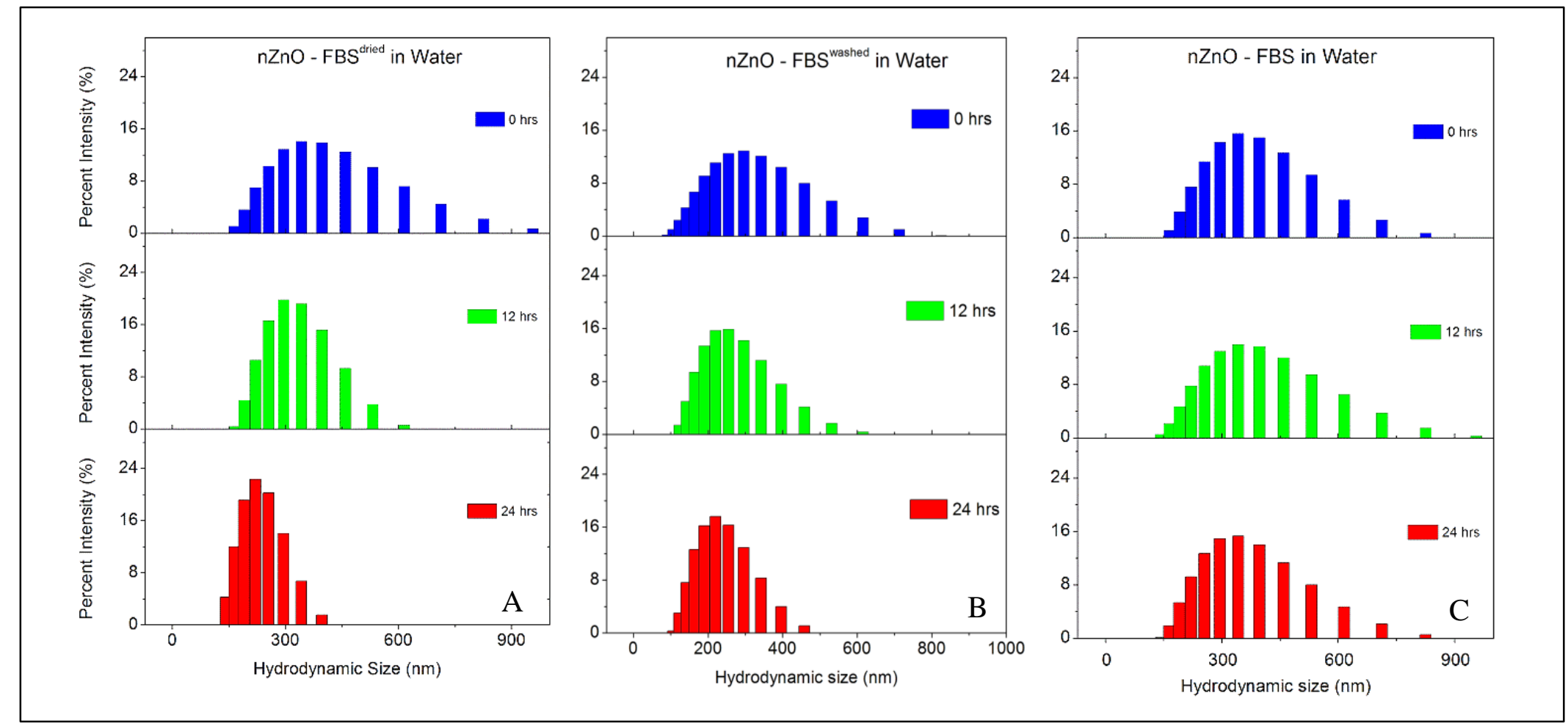

Figure 2.7 Hydrodynamic size distribution profiles at 0,12 and 24 hours. Histograms represent $25 \mathrm{mM}$ stock dispersions prepared in water for $\mathrm{A}$ ) $\mathrm{nZnO}$ - FBS ${ }^{\text {dried }}$, B) $\mathrm{nZnO}$ - FBS ${ }^{\text {washed }}$ and C) $\mathrm{nZnO}$ - FBS. 


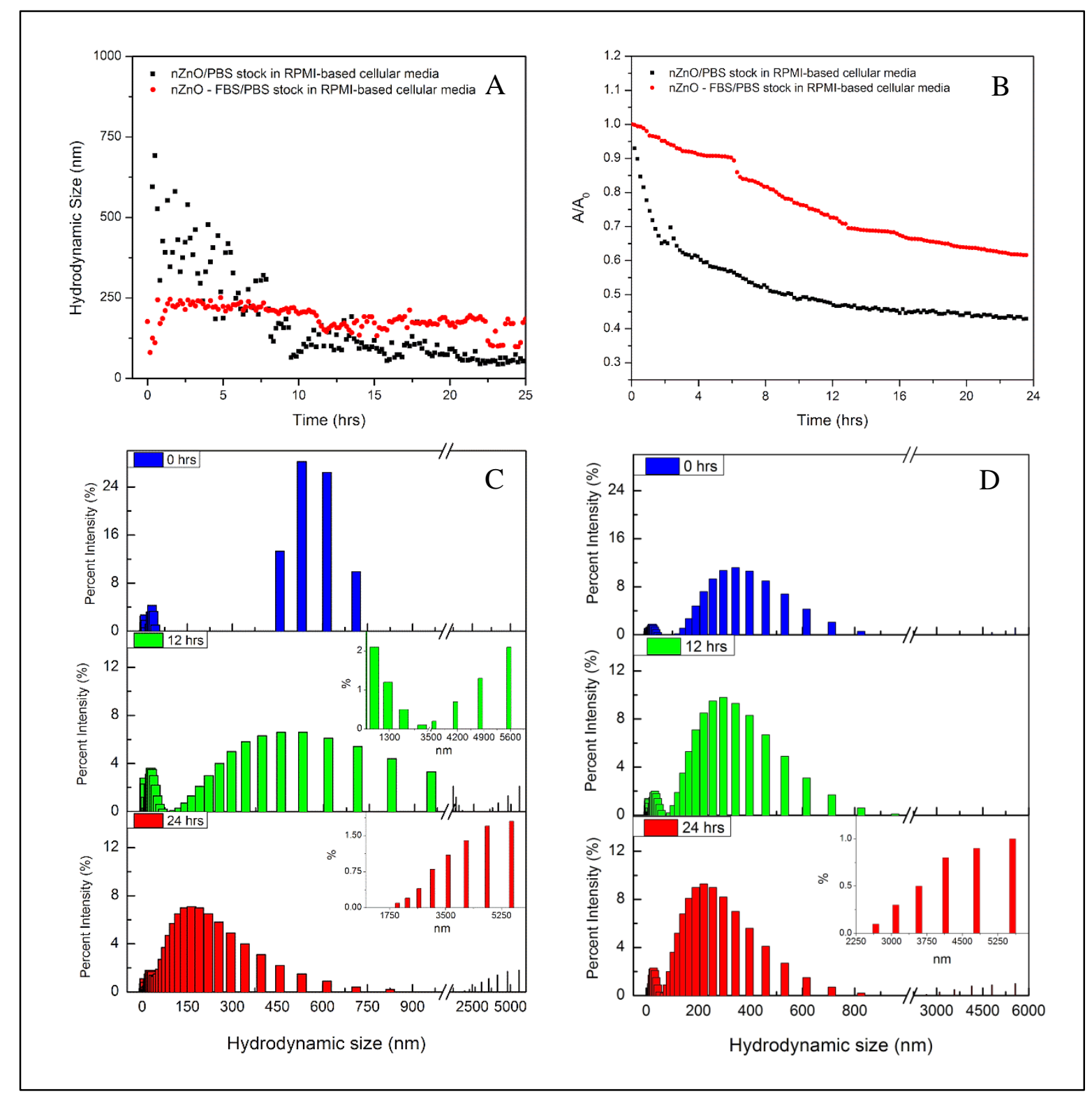

Figure 2.8 Dispersion stability analysis for $\mathrm{nZnO/PBS}$ and $\mathrm{nZnO}$ - FBS/PBS stock solutions introduced to RPMI-based cellular media at a concentration of $0.6 \mathrm{mM}$. A) Average hydrodynamic size profiles for a 24-hour time period. B) Sedimentation profiles for a 24-hour time period. Hydrodynamic size distribution profiles at 0,12 and 24 hours for $\mathrm{C}$ ) $\mathrm{nZnO} / \mathrm{PBS}$ stock dispersion in RPMI-based cellular media and D) nZnO - FBS/PBS stock dispersion in RPMI-based cellular media. 


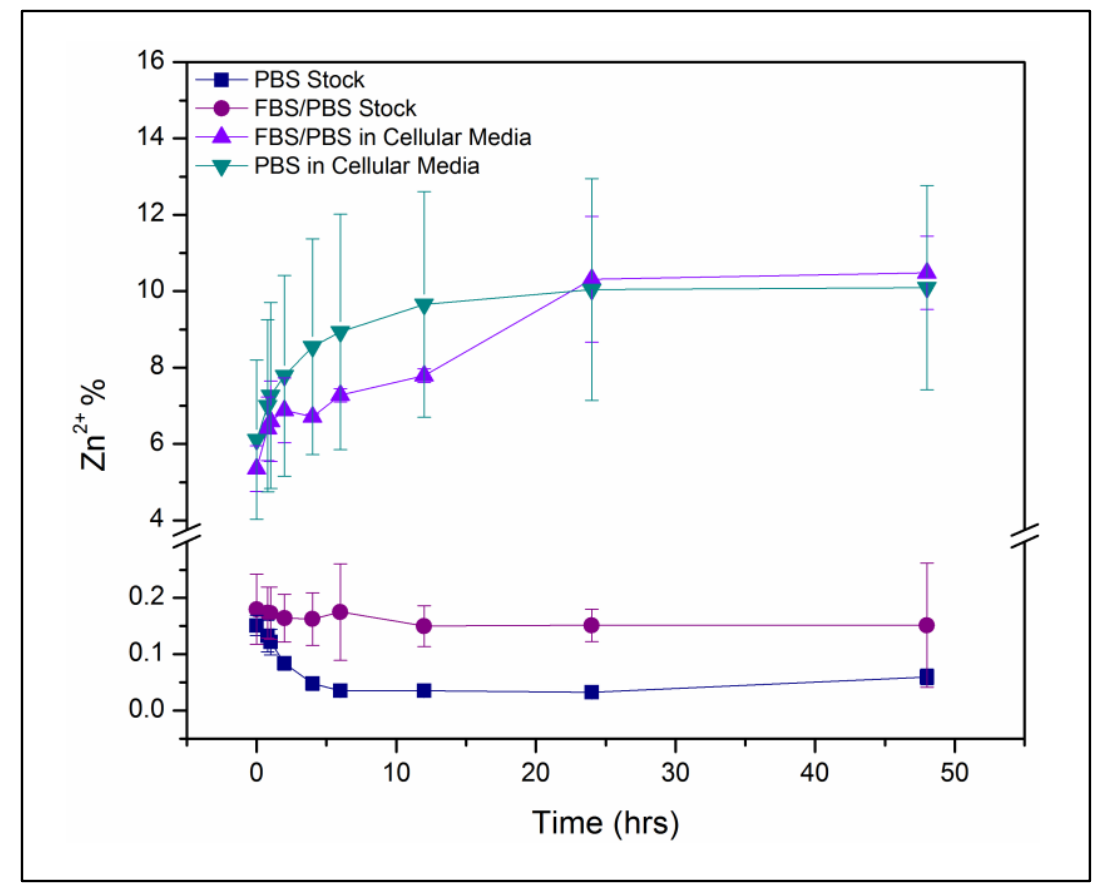

Figure 2.9 Extracellular dissolution of $6 \mathrm{mM} \mathrm{nZnO/PBS}$ and $\mathrm{nZnO}-$ FBS/PBS stock dispersions and $0.6 \mathrm{mM}$ of those respective $\mathrm{nZnO}$ stock dispersions in RPMIbased cellular media. $\mathrm{Zn}^{2+}$ concentration is expressed as $\% \mathrm{Zn}^{2+}$ (total dissolved $\mathrm{Zn}^{2+}$ measured by ICP-MS / total $\mathrm{Zn}^{2+}$ in sample) to normalize for concentration. 


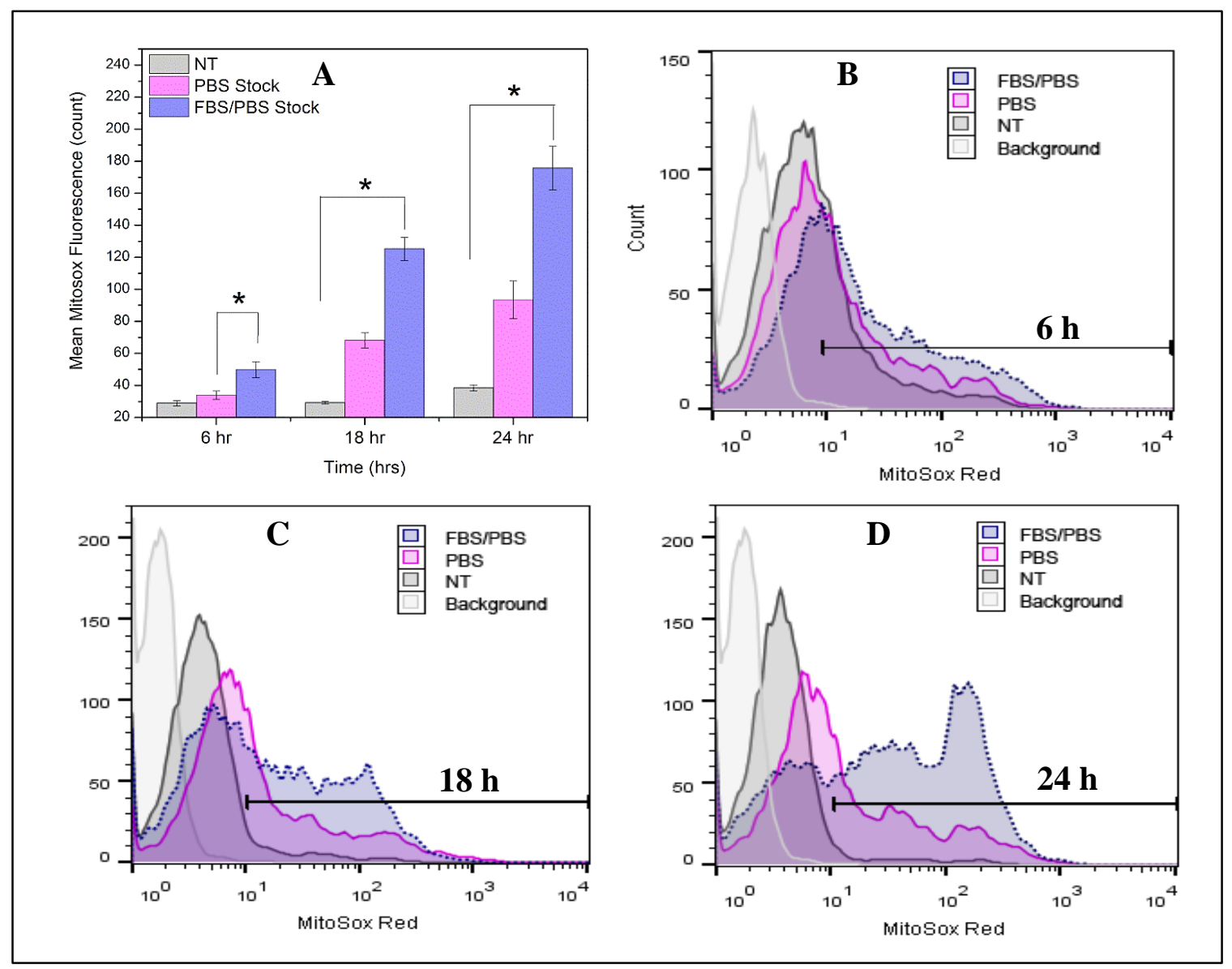

Figure 2.10 Cellular production of ROS following ZnO NP exposure. ROS generation was evaluated in Jurkat cells at 6,18 and 24 hours following treatment with nZnO/PBS (PBS) and nZnO - FBS/PBS (FBS/PBS) stock dispersions using MitoSOX red and flow cytometry (means \pm standard error, minimum of $n=3$ ). Cells were treated with $0.4 \mathrm{mM} \mathrm{nZnO}$ and stained with MitoSOX red and CD3 antibody after the desired time. A) Mean MitoSOX fluorescence intensity for a non-treated control (NT) and $\mathrm{nZnO}$ and $\mathrm{nZnO}$-FBS exposure. To assess relative increases in ROS following NP treatment, a marker (M1) was set so that background fluorescent in control samples (MitoSOX loaded/no NP) was between 1 and $3.5 \%$. Histograms represent B) 6 hours, C) 18 hours and D) 24 hours. 

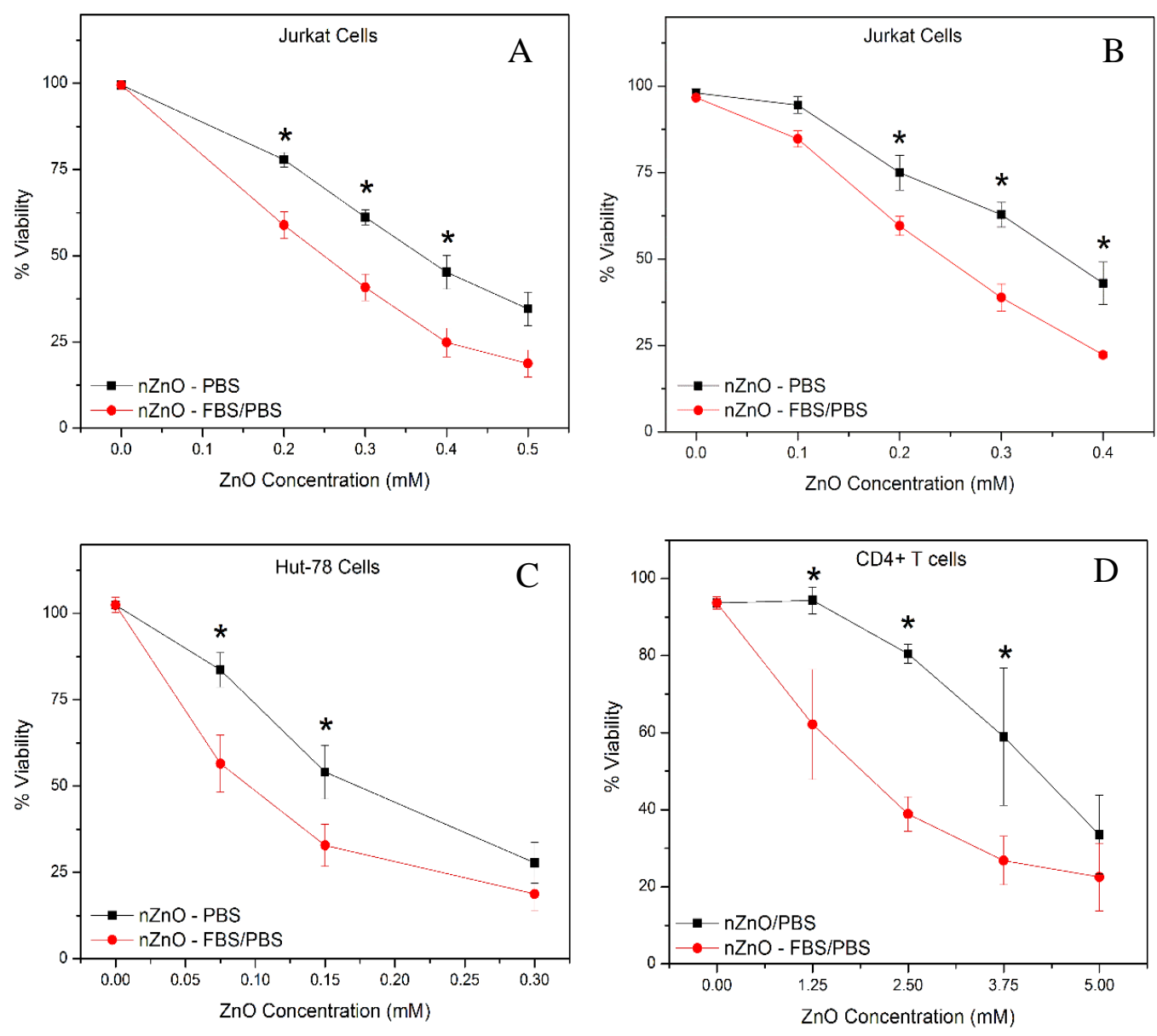

Figure 2.11 ZnO NP toxicity using nZnO/PBS and nZnO-FBS/PBS stock dispersions for 24 hours on various suspension cell types. Cultures were treated concurrently with varying concentrations of $\mathrm{ZnO}$ NP for 24 hours and cell viability was evaluated (means \pm standard error, minimum of $n=3$ ). Statistical analysis was performed using repeated measures analysis of variance and model-based means post hoc test $(p<0.05)$ with an asterisk denoting statistical significance. Jurkat cell viability was assessed using both (A) alamar blue staining or (B) flow cytometry and PI uptake to evaluate between assay variability and repeatability. (C) Hut-78 cell viability using alamar blue staining and (D) $\mathrm{CD4}^{+} \mathrm{T}$ Cell viability using flow cytometry and PI uptake. 

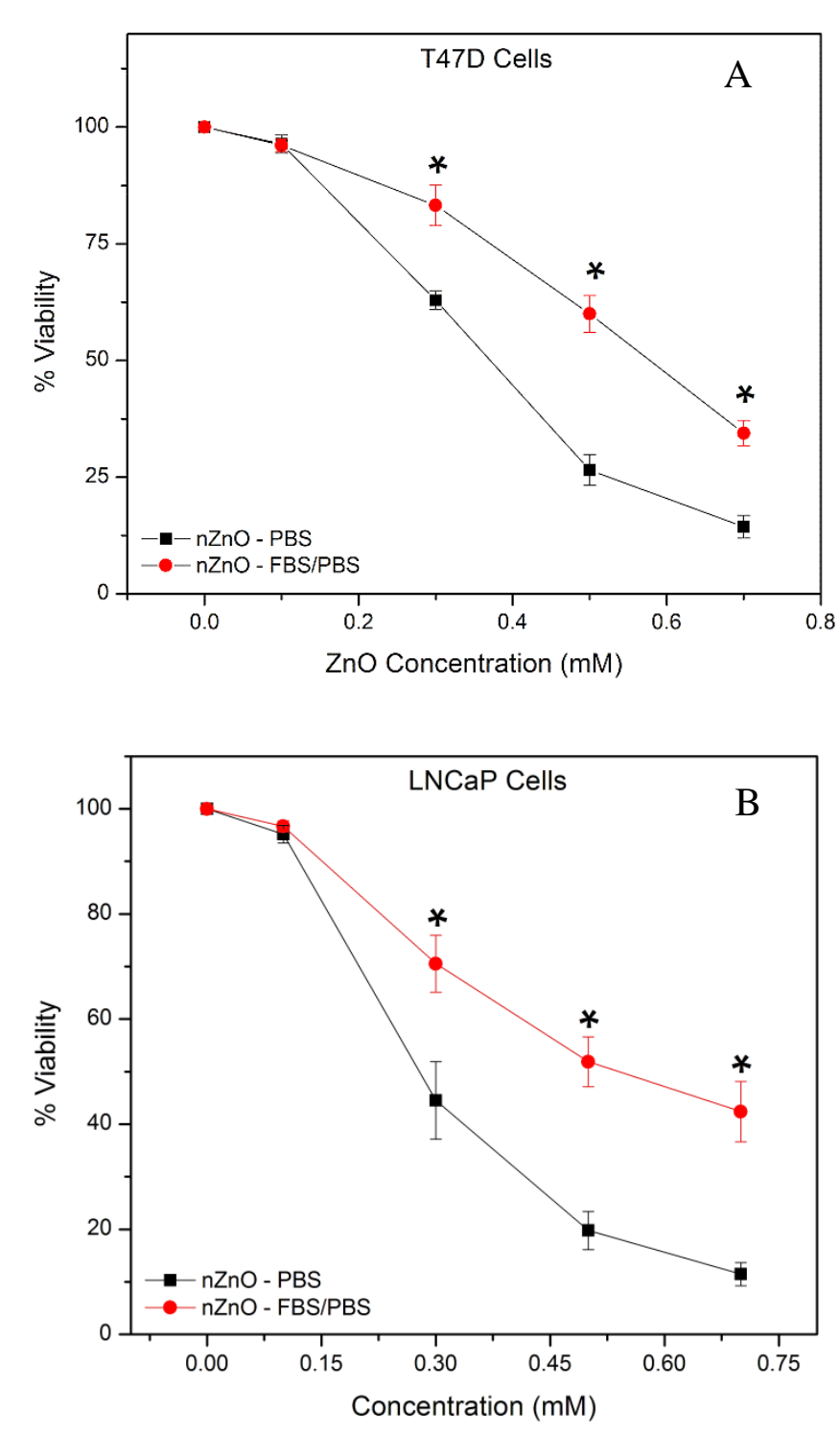

Figure 2.12 ZnO NP toxicity using varying concentrations of $\mathrm{nZnO} / \mathrm{PBS}$ and $\mathrm{nZnO}$ - FBS/PBS stock dispersions for 24 hours on adherent cell types. (A) T-47D epithelial mammary gland carcinoma and (B) LNCaP epithelial prostate cancer cells. Cultures were treated concurrently and cell viability was evaluated (means \pm standard error, minimum of $n=3$ ). Statistical analysis was performed using repeated measures analysis of variance and model-based means post hoc test $(p<0.05)$ with an asterisk denoting statistical significance. 


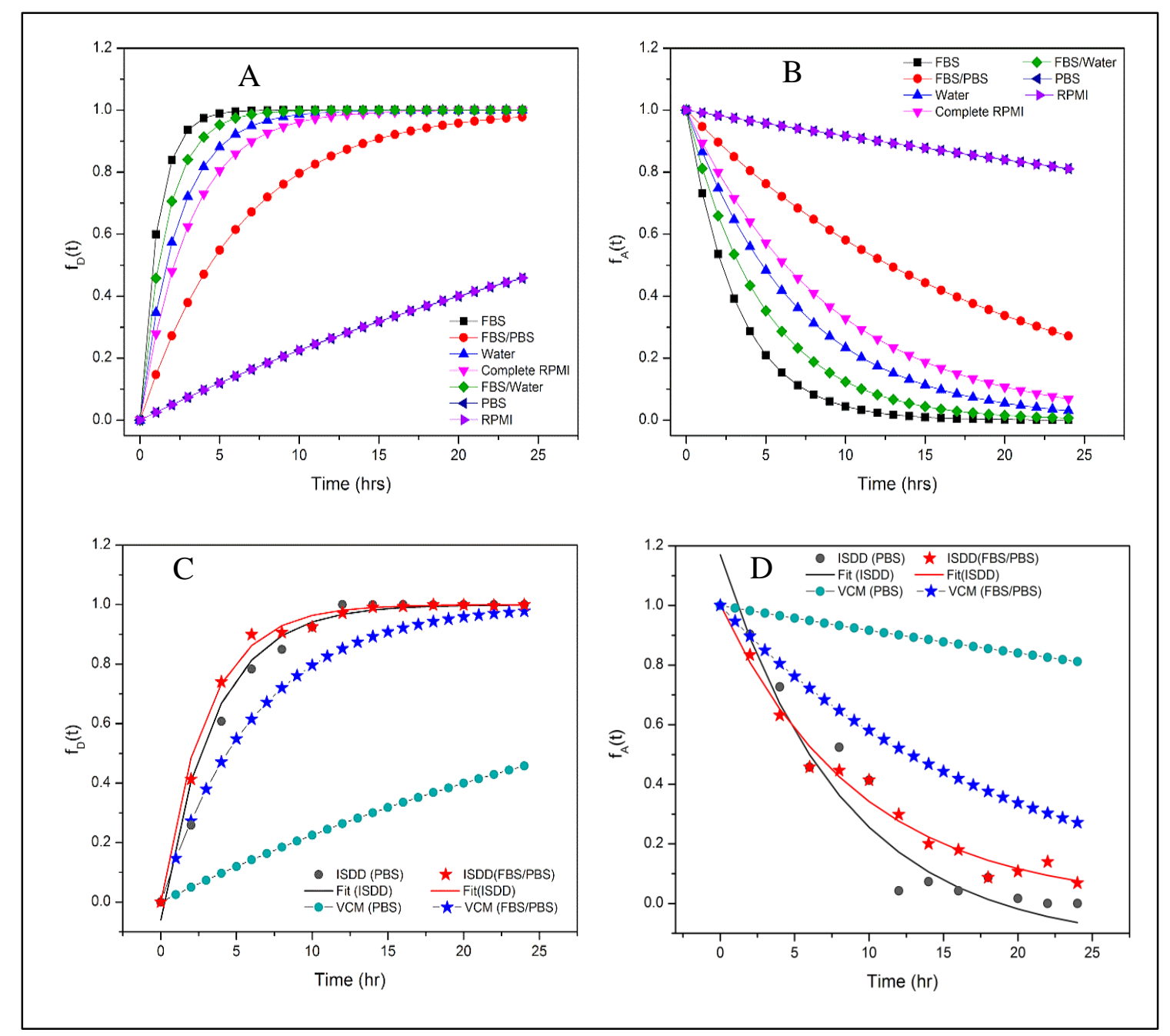

Figure 2.13 Dosimetry curves for various stock dispersions introduced to RPMIbased cellular media at a concentration of $1.25 \mathrm{mM}$. A) Curves constructed using $\alpha$ values calculated for 24-well plates and representing the fraction of NP deposited $\left[f_{D}(t)\right]$ to an adherent cell as a function of time. B) Curves constructed using $\alpha$ values calculated for 96-well plates and representing the fraction of NPs available $\left[f_{A}(t)\right]$ to cells grown in suspension as a function of time. Dosimetry curves constructed using both the Harvard VCM and ISDD models for both the nZnO/PBS and nZnO FBS/PBS stocks representing C) NP deposition $\left[f_{D}(t)\right]$ and D) NP availability $\left[f_{A}(t)\right]$. 


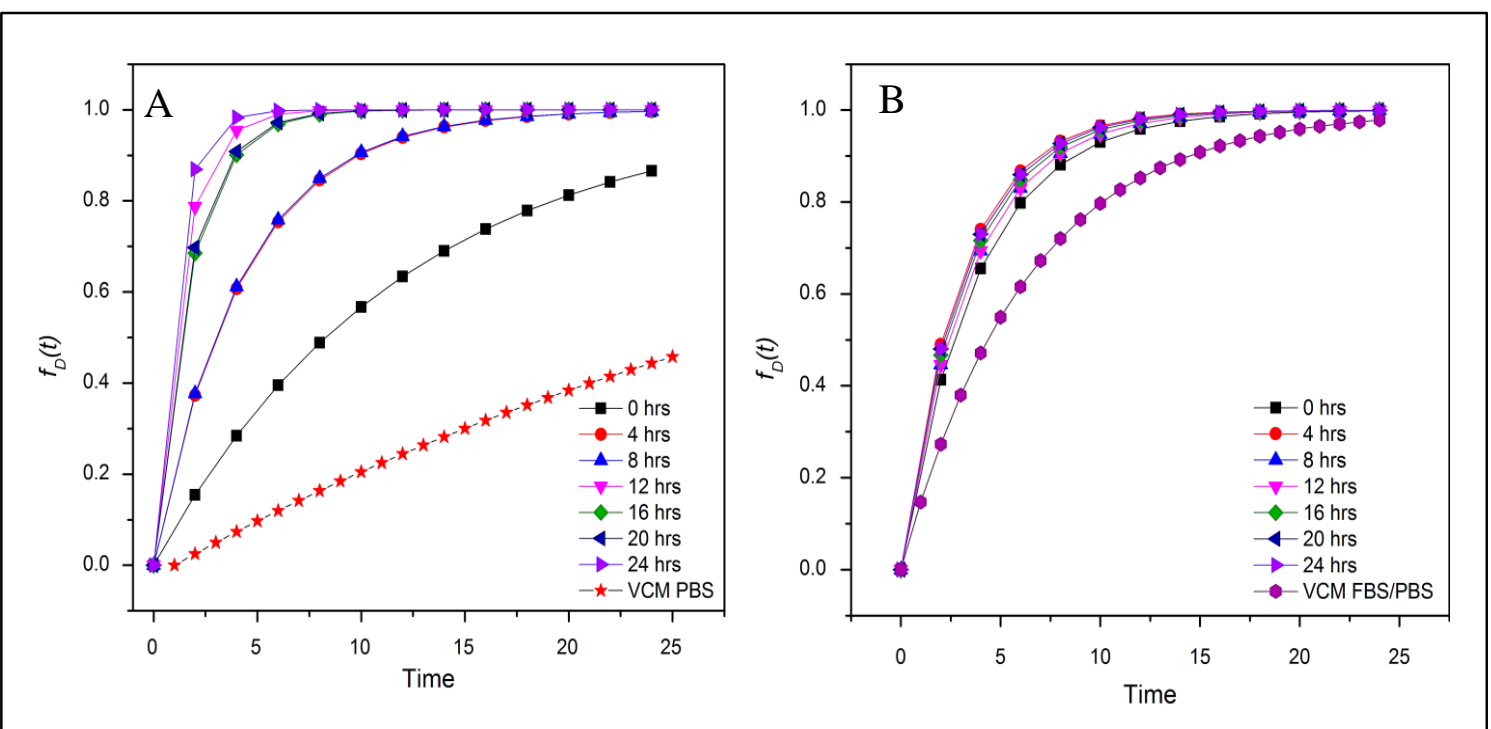

Figure 2.14 Dosimetry curves constructed using both the Harvard VCM and ISDD models for nZnO/PBS and $\mathrm{nZnO}$ - FBS/PBS stock dispersions introduced to RPMIbased cellular media at a concentration of $1.25 \mathrm{mM}$. Curves were constructed using $\alpha$ values calculated for 24 -well plates at various time points (ISDD) and Harvard VCM $\alpha$ values (Table 1). These curves represent the fraction of NPs deposited $\left[f_{D}(t)\right]$ to an adherent cell layer as a function of time for A) $\mathrm{nZnO}$ - PBS stock dispersions and $\mathrm{B}$ ) $\mathrm{nZnO}$ - FBS/PBS stock dispersions. 
Table 2.1 Delivered Dose Metrics.

\begin{tabular}{|c|c|c|c|c|c|c|c|}
\hline \multirow[t]{2}{*}{$\begin{array}{l}\text { Stock Dispersion in } \\
\text { complete RPMI }\end{array}$} & \multirow[t]{2}{*}{$\begin{array}{l}\text { Zeta Potential } \\
\qquad(\mathrm{mV})\end{array}$} & \multirow[t]{2}{*}{$d_{H}(\mathrm{~nm})$} & \multirow[t]{2}{*}{$p_{e}\left(\mathrm{~g} / \mathrm{cm}^{3}\right)$} & \multicolumn{2}{|c|}{$\begin{array}{l}\text { 96-well plate } \\
(\mathrm{L}=6.6 \mathrm{~mm})\end{array}$} & \multicolumn{2}{|c|}{$\begin{array}{l}\text { 24-well plate } \\
(\mathrm{L}=2.25 \mathrm{~mm})\end{array}$} \\
\hline & & & & $\alpha$ & $\mathrm{t}_{90}(\mathrm{~h})$ & $\alpha$ & $\mathrm{t}_{90}(\mathrm{~h})$ \\
\hline Water & $-16.1 \pm 1.45$ & 182.5 & 2.021 & 0.1453 & 15.6 & 0.4261 & 5.4 \\
\hline FBS/Water & $-15.1 \pm 0.81$ & 185.1 & 1.692 & 0.2085 & 11.1 & 0.6116 & 3.8 \\
\hline FBS & $-13.9 \pm 0.40$ & 185.5 & 1.464 & 0.3121 & 7.4 & 0.9154 & 2.5 \\
\hline PBS & $-8.1 \pm 1.93$ & 663 & 1.879 & 0.0087 & 264.9 & 0.0255 & 90.3 \\
\hline FBS/PBS & $-9.8 \pm 2.08$ & 322 & 1.743 & 0.0543 & 42.4 & 0.1593 & 14.5 \\
\hline RPMI & $-14.7 \pm 1.46$ & 770.5 & 1.623 & 0.0087 & 263.8 & 0.0256 & 89.9 \\
\hline Complete RPMI & $-13.6 \pm 2.63$ & 244.4 & 1.682 & 0.1116 & 20.6 & 0.3273 & 7.1 \\
\hline
\end{tabular}

Delivered dose metrics: $p_{e}$ : agglomerate effective density $\left(\mathrm{g} / \mathrm{cm}^{3}\right) ; d_{H}$ : Hydrodynamic diameter (nm); L: media height; $\alpha$ : deposition fraction; t90: Time required for delivery of $90 \%$ of administered dose $(\mathrm{h}) ; \alpha$ and $\mathrm{t}_{90}(\mathrm{~h})$ values are designated for each well plate employed (96-well plate for suspension cells and 24-well plate for adherent cells). 


\section{CHAPTER 3 ZNO NANOPARTICLE PREPARATION ROUTE INFLUENCES SURFACE REACTIVITY, DISSOLUTION AND CYTOTOXICITY}

Catherine B. Anders, ${ }^{a}$ Josh E. Eixenberger, ${ }^{a}$ Nevil A. Franco, ${ }^{\text {b }}$ Rebecca J. Hermann, ${ }^{\text {a }}$ Katherine D. Rainey, ${ }^{\mathrm{b}}$ Jordan J. Chess, ${ }^{\mathrm{d}}$ Alex Punnoose, ${ }^{\mathrm{b}}$ and Denise G. Wingett *ac

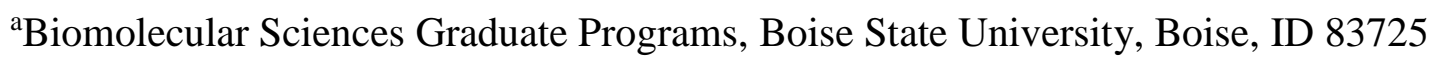

${ }^{\mathrm{b}}$ Department of Physics, Boise State University, Boise, ID 83725

${ }^{\mathrm{c}}$ Department of Biological Sciences, Boise State University, Boise, ID 83725

${ }^{d}$ Department of Physics, University of Oregon, Eugene, OR 97403

*Address correspondence to Denise G. Wingett, email; denisewingett@ boisestate.edu

Citation: Anders, C.B., Eixenberger, J.E., Franco, N.A., Hermann, R.J., Rainey, K.D., Chess, J.J., Punnoose, A., and Wingett, D.G. ZnO Nanoparticle Preparation Route Influences Surface Reactivity, Dissolution and Cytotoxicity. Environmental Science: Nano, 2018, DOI: 10.1039/C7EN00888K.

Publisher Springer US

Accepted: January 5, 2018

Changes No significant changes were made to this publication. 


\begin{abstract}
$\mathrm{ZnO}$ nanoparticles $(\mathrm{nZnO})$ are commonly used in nanotechnology applications despite their demonstrated cytotoxicity against multiple cell types. This underscores the significant need to determine the physicochemical properties that influence $\mathrm{nZnO}$ cytotoxicity. In this study, we analyzed six similarly sized nZnO formulations, along with $\mathrm{SiO}_{2}$-coated $\mathrm{nZnO}$, bulk $\mathrm{ZnO}$ and $\mathrm{ZnSO}_{4}$ as controls. Four of the $\mathrm{nZnO}$ samples were synthesized using various wet chemical methods, while three employed high-temperature flame spray pyrolysis (FSP) techniques. X-ray diffraction and optical analysis demonstrated the lattice parameters and electron band gap of the seven $\mathrm{nZnO}$ formulations were similar. However, zeta potential measures, hydrodynamic size, photocatalytic rate constants, dissolution potential, reactive oxygen species (ROS) production and, more importantly, the cytotoxicity of the variously synthesized nZnO towards Jurkat leukemic and primary $\mathrm{CD}^{+} \mathrm{T}$ cells displayed major differences. Surface structure analysis using FTIR, X-ray photoelectron spectroscopies (XPS) and dynamic light scattering (DLS) revealed significant differences in the surface-bound chemical groups and the agglomeration tendencies of the samples. The wet chemical $\mathrm{nZnO}$, with higher cationic surface charge, faster photocatalytic rates, increased extracellular dissolution and ROS generation demonstrated greater cytotoxicity towards both cell types than those made with FSP techniques. Furthermore, principal component analysis (PCA) suggests that the synthesis procedure employed influences which physicochemical properties contribute more to the cytotoxic response. These results suggest that the synthesis approach results in unique surface chemistries and can be a determinant of cellular cytotoxicity and oxidative stress responses.
\end{abstract}




\section{Introduction}

Zinc oxide nanoparticles $(\mathrm{nZnO})$ are widely used in industrial processes, electronics and consumer products due to their unique optical, electronic, and structural properties that are absent in bulk $\mathrm{ZnO}$ (around one micron in size) [1-3]. Given this, considerable research has centred around the inherent toxicity that $\mathrm{nZnO}$ demonstrates towards various organisms [4, 5], as well as prokaryotic [6-10] and eukaryotic cells $[9,11$ 19]. While many researchers have worked to engineer safer-by-design nanomaterials through material properties modification [20-22], others have sought to exploit this differential toxicity for potential use in nZnO-based therapeutics $[9,11,12]$. Central to both of these long-term objectives are a comprehensive examination of nZnO-specific physicochemical properties and how material property modifications influence oxidative stress and nanoparticle (NP)-induced toxicity. Despite the wealth of available research, however, clear mechanistic determinants of these responses remain elusive. Among the proposed mechanisms is that intrinsic ROS production originating from redox reactions on the NP surface contributes directly to cellular membrane, organelle, or DNA damage [2325]. Alternatively, perturbations in intracellular zinc homeostasis from NP dissolution, proinflammatory responses and mitochondrial damage leading to ROS production from the cell death process have also been implicated [26-32]. Regardless, controlling reactivity at the NP-surface and $\mathrm{nZnO}$ dissolution is critical to influencing biological outcomes.

Most studies investigate changes in NP-induced toxic response as a function of a single material property modification in a specific $\mathrm{nZnO}$ formulation. For example, minor changes within the same synthesis method can alter both the crystal size and shape [25]. Likewise, surface modification via surface coating, chemical modification, or heat 
treatment has demonstrated efficacy in changing surface charge, surface structure and NP solubility [23-25, 33, 34]. While this formulation controlled approach has been instrumental in identifying how specific physicochemical modifications influence cellular responses, it fails to explain how comparable studies using similar NP modifications and/or mammalian cell types, but differently synthesized nZnO samples, often result in differing cytotoxicity. These discrepancies have resulted in uncertainty as to how changes in material properties, within a very diverse set of $\mathrm{nZnO}$ formulations, influence the toxicity of $\mathrm{nZnO}$.

Studies in bacterial models routinely employ step-wise synthesis method changes to improve $\mathrm{nZnO}$ photocatalytic activity and antimicrobial properties [6-8, 10, 35-40]. However, similar studies in mammalian cells remain an underrepresented area in $\mathrm{nZnO}$ research. In previous work, our lab compared two spherical and similarly sized $(\sim 9 \mathrm{~nm})$ pristine $\mathrm{nZnO}$ formulations fabricated using different wet-chemical methods [25]. Despite these similarities, the two formulations exhibited different (i) hydrodynamic size, (ii) electrophoretic mobility, (iii) isoelectric point (IEP), (iv) surface-adsorbed groups, (v) photocatalytic activity, and (vi) remarkably different cytotoxicity. This work illustrates the importance of investigating how changes to the synthesis method can dramatically alter the surface reactivity, and thus, NP-induced toxicity.

Additionally, there is still much debate as to the roles that extracellular $[13,30]$, intracellular free $[29,31]$, and total cell-associated zinc $[13,31]$ play in the mechanistic process of NP-induced toxicity. Once introduced into cell culture, $\mathrm{nZnO}$ will begin to dissolve into ionic zinc (shown as extracellular zinc in Scheme 3.1). Inevitably, some of this ionic $\mathrm{Zn}^{2+}$ will be taken up by cells to become intracellular free $\mathrm{Zn}^{2+}$ within the cytosol or endocytotic vesicles $[32,41,42]$. Additionally, $\mathrm{Zn}^{2+}$ ions will interact with carbonate 
and phosphate anions to produce zinc carbonate or phosphate species [43]. While these species show negligible toxicity towards mammalian cells $[44,45]$, they do interact with large agglomerates of intact $\mathrm{nZnO}$ (i.e., non-ionic zinc) to form amorphous complexes of encapsulated crystalline zinc that freely attach to cellular membranes or enter cells through various uptake mechanisms (designated as cell-associated $\mathrm{Zn}^{2+}$ in Scheme 3.1) $[17,31,41$, 45]. Consequently, a thorough examination of these processes is critical in NP-toxicology studies.

In this work, we sought to expand upon our previous studies by evaluating a more diverse panel of $\mathrm{nZnO}$ formulations with differential toxicity to include four wet-chemical samples, two FSP prepared samples, and three control samples, specifically $\mathrm{ZnSO}_{4}$, bulk $\mathrm{ZnO}$, and $\mathrm{a} \mathrm{SiO}_{2}$-coated $\mathrm{nZnO}$ formulation. Careful consideration was used in choosing the $\mathrm{nZnO}$ samples for investigation. Wet chemical methods are ubiquitously used in research studies as they are easily synthesized using inexpensive, common laboratory equipment and chemicals, and can be readily modified with simple experimental changes. The four wet chemical methods chosen are among the most popular approaches utilized. All employ a forced hydrolysis synthesis reaction with variable precursors and solvent systems. FSP synthesis is favored in industrial settings because of its simplistic methodology with fewer process steps, scalability, easy collection systems, high yield, and purity of the final product [46-51]. Additionally, FSP methods employ high temperatures that well exceed any temperatures employed by most wet chemical methods. The specific FSP methods were selected based on differing precursor solutions and carrier gas mixtures. The use of nanomaterials bridging both research application and consumer product use should provide NP representation across multiple areas of nZnO usage. All the nanoscale samples were 
found to have average sizes in at least one dimension between $16-45 \mathrm{~nm}$ with similar bandgaps centred near $3.3 \mathrm{eV}$. Extensive characterizations were performed to evaluate the physicochemical differences present in the surface properties of the samples, and explore the roles that extracellular zinc, free intracellular zinc, and cell-associated zinc play in cellular toxicity. By looking at a broad panel of pristine $\mathrm{nZnO}$ with variable toxic potential, we have identified how NP-induced toxicity is influenced by both the dissolution potential and the surface reactivity of $\mathrm{nZnO}$. This understanding could provide critical insight into previously unexplained discrepancies in the wider body of research as well as define which material property interactions have the greatest potential to affect cellular toxicity.

\section{Experimental}

\section{Synthesis of $\mathrm{nZnO}$}

Seven nZnO samples were used in this study. Four of the samples were synthesized using wet chemical methods. The DEG nZnO sample was produced using the forced hydrolysis of a zinc acetate dihydrate precursor in diethylene glycol (DEG). The solution was held at $150{ }^{\circ} \mathrm{C}$ for 90 minutes. Nanopure water was added to the solution at $80{ }^{\circ} \mathrm{C}$ to obtain the desired crystallite size [12]. Once cooled to room temperature, the $\mathrm{nZnO}$ were separated from solution via centrifugation and subsequent washings with ethanol.

The second wet chemical method, EtOH nZnO, was synthesized using a similar hydrolysis method with the same precursor, but denatured ethanol was used as the reaction solvent along with a strong base, $\mathrm{LiOH}$, to maintain appropriate $\mathrm{pH}$ [25]. The solution was held at $80{ }^{\circ} \mathrm{C}$ for 90 minutes, cooled to room temperature, and aged with n-heptane for several hours. The NPs were removed from suspension via centrifugation, followed by 
subsequent washings with nanopure water and ethanol. After washings, both the DEG and $\mathrm{EtOH} \mathrm{nZnO}$ precipitates were dried in an oven at $65^{\circ} \mathrm{C}$ for $>12$ hours.

The third sample, EG nZnO, was synthesized using a wet chemical method followed by a thermal decomposition. Zinc acetate dehydrate was used as the precursor with polyvinylpyrrolidone (PVP) as a binding agent. The reaction was carried out by suspending the precursor and PVP in ethylene glycol (EG), and held at $180{ }^{\circ} \mathrm{C}$ for 90 minutes. After cooling to room temperature, the particles were collected by centrifugation, followed by ethanol washings. The sample pellet was dried overnight at $65{ }^{\circ} \mathrm{C}$ and then annealed at $350{ }^{\circ} \mathrm{C}$ for 2 hours to remove any residual organics.

The final wet chemical sample, $\mathrm{NaOH} \mathrm{nZnO}$, was synthesized by introducing a 0.5 $\mathrm{M}$ zinc nitrate $\left[\mathrm{Zn}\left(\mathrm{NO}_{3}\right)_{2} \cdot 6 \mathrm{H}_{2} \mathrm{O}\right]$ precursor solution dropwise to an equal volume of $1.0 \mathrm{M}$ $\mathrm{NaOH}$ heated to $80{ }^{\circ} \mathrm{C}$ [52]. This solution was continuously stirred for two hours. The resulting precipitate was removed from solution via centrifugation and repeatedly washed with ethanol and nanopure water, and dried at $65^{\circ} \mathrm{C}$ for $>12$ hours.

The three remaining $\mathrm{nZnO}$ samples were prepared using high-temperature flame spray pyrolysis (FSP). Rod-shaped FSP NPs (FSPR) and a silica-coated formulation of the FSPR sample $\left(\mathrm{SiO}_{2}\right.$-FSPR) were prepared by collaborators at the Department of Environmental Health, Harvard University. Both samples employed a precursor solution of $0.5 \mathrm{M}$ zinc naphthenate dissolved in ethanol for the formation of the $\mathrm{nZnO}$ core with the addition of hexamethyldisiloxane (HMDSO) utilized to form the silica coating. Specific synthesis details have been previously reported [21, 22]. A spherical FSP sample (FSPS) was synthesized by the Nanoscience Research Laboratory at Chiang Mai University in Thailand as outlined in Liewhiran et al. (2007) [53]. For this formulation, zinc naphthenate 
was dissolved in a toluene /acetonitrile mixture with a ratio of $80 / 20 \mathrm{vol} \%$ at a precursor concentration of $0.5 \mathrm{M} \mathrm{ZnO}$. Lastly, a bulk $\mathrm{ZnO}$ sample was obtained by annealing a commercially available $\mathrm{ZnO}$ powder (Sigma Aldridge; St. Louis, MO) at $500{ }^{\circ} \mathrm{C}$ for 5 hours to ensure all nanosized particles less than $200 \mathrm{~nm}$ were removed from the sample.

$\underline{\text { Characterization }}$

X-ray diffraction (XRD), transmission electron microscopy (TEM), dynamic light scattering (DLS), zeta potential, UV-vis spectrophotometry, and Fourier transform infrared spectroscopy (FTIR) were employed to evaluate chemical composition, crystal size, morphology, electrophoretic mobility and surface structure. Philips X'Pert and Rigaku Miniflex 600 X-ray diffractometers with a $\mathrm{Cu} \mathrm{K}_{\mathrm{a}}$ source $(1=1.5418 \AA$ ) $)$ in Bragg-Brentano geometry were used to record room temperature XRD spectra. Rietveld refinement using Materials Analysis Using Diffraction (MAUD) software corrected for instrumental broadening was used to determine crystal size and lattice parameters [54]. An FEI Tecnai transmission electron microscope operating at $120 \mathrm{kV}$ was employed to collect TEM images. High-resolution TEM analysis was performed on an FEI Titan microscope operating at $300 \mathrm{kV}$. Image processing was carried out using the Digital Micrograph software from Gatan (Pleasant, CA). The average particle size and the standard deviation of the nanopowders was determined by measuring 100 plus individual NPs within Image J software (NIH, Bethesda, MD) [55].

The specific surface areas of the samples were determined via nitrogen gas physiosorption at $77 \mathrm{~K}$ using a Quantachrome NOVA 2200e analyzer. Prior to the analysis, all samples were vacuum degassed at $250{ }^{\circ} \mathrm{C}$ for 3.5 hours. The linear portion 
$\left(P / P_{\mathrm{o}}=0.05-0.3\right)$ of the Brunauer-Emmett-Teller (BET) model was used for the calculation of specific surface area with NovaWin Version 11.04 software.

Room-temperature (RT) ultraviolet and visible light optical absorbance spectra were obtained with a CARY 5000 spectrophotometer. RT diffuse reflectance UV-vis spectra were collected for band gap determination, and RT UV-vis absorbance spectra were obtained for photocatalytic rate analysis. Electrophoretic mobility and hydrodynamic sizes of $\mathrm{nZnO}$ dispersions created in nanopure water and cellular media were collected with a Malvern Zetasizer® NanoZS. Each formulation was measured three times using freshly prepared NP dispersions and a minimum of six replicates were collected for each measurement. FTIR spectra were measured using a Bruker Tensor 27 spectrometer.

\section{Cell culture and cytotoxicity studies.}

Cells were cultured at $37{ }^{\circ} \mathrm{C}$ and $5 \% \mathrm{CO}_{2}$. For cell cytotoxicity assays, Jurkat leukemia T cells were cultured in supplemented Roswell Park Memorial Institute (RPMI)1640 media (10 \% FBS, 2 mM L-glutamine, 1.5 g/L sodium bicarbonate, 4.5 g/L glucose, $10 \mathrm{mM}$ HEPES, $1.0 \mathrm{mM}$ sodium pyruvate, and $1 \%$ penicillin-streptomycin) and maintained in log phase prior to treatment. Stock solutions for the $\mathrm{nZnO}$ samples were created by suspending the nanomaterial in nanopure water and sonicating for 30 minutes using a power of $1.05 \mathrm{~W}$. These sonication parameters deliver a determined sonication energy [56] of $181 \mathrm{~J} / \mathrm{mL}$ needed to obtain stable $\mathrm{nZnO}$ dispersions [57]. Working stock dilutions were prepared by introducing the NP stock to cellular media and vortex mixing for 30 seconds. The NPs were then added to cells ( $5 \times 10^{5}$ cells $\left./ \mathrm{mL}\right)$ and cultured for 24 hours. $\mathrm{ZnSO}_{4}$ was utilized as a positive ionic zinc control. Cell viability was determined using a metabolic assay in which alamar blue (10\% of total volume) was added to cells 
and incubated for an additional 4 hours. Fluorescence changes were measured with a Biotek Synergy MX ${ }^{\circledR}$ plate reader (Winooski, VT) using an excitation of $530 \mathrm{~nm}$ and emission collection at $590 \mathrm{~nm}$.

Additional studies were performed using human primary $\mathrm{CD}^{+} \mathrm{T}$ cells. Written informed consent was obtained from all blood donors and the study was approved by the University Institutional Review Board. Ficoll-Hypaque density centrifugation (Histopaque-1077, Sigma Aldridge; St. Louis, MO) was used to isolate peripheral blood mononuclear cells from normal human blood samples followed by negative immunomagnetic selection (Stem Cell Technologies; Vancouver, BC, Canada) to obtain $\mathrm{CD}^{+} \mathrm{T}$ cells $[11]$. Purified $\mathrm{CD}^{+}{ }^{+} \mathrm{T}$ cells $(>95 \%$ purity) were cultured at a concentration of $1 \times 10^{6}$ cells $/ \mathrm{mL}$ in RPMI with $10 \%$ FBS. Cells $\left(1 \times 10^{6}\right.$ cells $\left./ \mathrm{ml}\right)$ were treated with NPs for 24 hours. Cell were then stained with a fluorescein isothiocyanate (FITC)-labelled mouse anti-human HLA ABC antibody (Clone: G46-2.6; BD Bioscience; San Jose, CA) $0.04 \mu \mathrm{g} / \mathrm{mL}$ propidium iodide (PI) at $4{ }^{\circ} \mathrm{C}$ for 30 minutes. A BD FACSCalibur flow cytometer (BD Biosciences; San Jose, CA) was used to monitor the loss of cell membrane integrity, and light forward scatter (FSC) and side scatter (SSC) parameters were used to exclude any NPs from the analysis. Additionally, each sample was treated with $10 \mu \mathrm{L}$ of fluorescent CountBright counting beads (Invitrogen; Carlsbad, CA) to enable determinations of absolute cell numbers.

\section{$\underline{\text { Photocatalytic Studies }}$}

The photocatalysis method employed was adapted with changes from Punnoose, et al. (2015) [25]. Using the model fluorescent dye sulfo-Rhodamine B, $\mathrm{nZnO} /$ dye solutions were prepared by adding $2 \mathrm{mg}$ of nanomaterial in $1 \mathrm{~mL}$ of nanopure 
water, sonicating for 15 minutes, and then introduced into $20 \mathrm{~mL}$ of 2.5 x 10-5 M solution of sulfo-Rhodamine B dye (excitation/emission maxima 565/586 nm) The nZnO/dye solution was equilibrated in the dark at room temperature with continuous stirring for 15 minutes. After reaching equilibrium, an aliquot was removed for UV-Vis analysis. The $\mathrm{nZnO/dye} \mathrm{solution} \mathrm{was} \mathrm{then} \mathrm{placed} \mathrm{in} \mathrm{a} \mathrm{Rayonet} \mathrm{RPR-100} \mathrm{UV} \mathrm{reactor} \mathrm{equipped} \mathrm{with} 2537$ A UV lamps to deliver approximately $3.2 \mathrm{~mW} / \mathrm{cm} 2$. Once the lamps were turned on, another sample was immediately extracted for analysis for the zero-minute time point. Aliquots were then removed every 15 minutes for a period of 120 minutes or until complete dye degradation. All liquid aliquots were centrifuged at $13,000 \times \mathrm{g}$ for 90 seconds to separate NPs from the solution. The supernatant was then transferred to a quartz cuvette, and optical absorbance was measured using a Cary-5000 UV-vis spectrophotometer. The photocatalytic rate constant $\mathrm{k}$ is given by $\mathrm{kt}=\ln \left(\mathrm{C}_{0} / \mathrm{C}\right)$, where $\mathrm{t}=$ time (minutes), $\mathrm{C}_{0}=$ initial concentration of fluorescent dye molecules, and $\mathrm{C}=$ concentration of the fluorescent dye molecules following its illumination with the NP sample. As a control, the selfdegradation of sulfo-Rhodamine B under UV irradiation without the presence of ZnO NPs was measured prior to $\mathrm{nZnO}$ studies and was found to be negligible.

$\underline{\text { Extracellular dissolution of } \mathrm{nZnO} \text { stock solutions }}$

To simulate cellular assay conditions, aliquots of NP stocks were added to cellular media. These suspensions were placed in $25 \mathrm{~mL}$ culture flasks at a fluid depth of $6.6 \mathrm{~mm}$ to mimic the dispersion dynamics experienced by $\mathrm{nZnO}$ in the 96 well cellular assays and were kept in an incubator. Two $\mathrm{mL}$ aliquots were removed at 4 and 24 hours and centrifuged at 5,000 x g for 20 minutes using an Amicon ${ }^{\mathrm{TM}}$ Ultra-4 Centrifugal Filter Unit with a $3-\mathrm{Kd}$ molecular weight cutoff $(0.1 \mathrm{~nm}$ pore size $)$ to remove any undissolved $\mathrm{nZnO}$ 
while allowing free zinc ions to pass through. Control experiments were performed to demonstrate that $99 \%$ NP-derived zinc ions are recoverable using this methodology [34]. Quantitative analysis of the dissolved $\mathrm{Zn}^{2+}$ ions was conducted on a Thermo $\mathrm{X}$ Series 2 quadrupole inductively-coupled plasma mass spectrometer (ICP-MS) under normal operating conditions (i.e., no CCT) with the $\mathrm{X}_{\mathrm{t}}$ cone set. Instrument performance was evaluated and optimized for each run. The instrument was calibrated using multi-element calibration standards containing $\mathrm{Zn}, \mathrm{Cr}, \mathrm{Mn}, \mathrm{Fe}, \mathrm{Co}$ and $\mathrm{Ni}$ in $2 \% \mathrm{HNO} 3$ at concentrations of approximately 1, 10, 100 and 1000 ppb. Instrument drift was monitored and corrected using $20 \mathrm{ppb}$ indium as an internal standard introduced online.

$\underline{\text { Intracellular } \mathrm{Zn}^{2+} \text { determination }}$

The amount of intracellular $\mathrm{Zn}^{2+}$ present in Jurkat cells post $\mathrm{nZnO}$ treatment was determined using the zinc specific dye FluoZin 3AM (Invitrogen; Eugene, OR). Jurkat cells were plated at $5 \times 10^{5}$ in 96-well plates and cultured with nZnO. At 4 and 24-hour time points, cells were harvested, rinsed twice with phosphate-buffered saline (PBS) and centrifuged at $500 \mathrm{x}$ g for $10 \mathrm{~min}$. The cell pellets were re-suspended in phosphate-free Dulbecco's Modified Eagle's medium (DMEM), stained with 3.25 $\mu$ M FluoZin 3AM dye and incubated at RT for 30 minutes in the dark. Cells were then rinsed with FACS buffer (PBS supplemented with $3 \%$ FBS and $0.02 \%$ sodium azide), centrifuged at $500 \mathrm{x}$ g for 7 min, re-suspended in FACS buffer, and stained with a FITC-labelled anti-HLA ABC antibody at RT for $30 \mathrm{~min}$ in the dark. Samples were then analyzed using a BD FACSCalibur flow cytometer with unbound NPs excluded from analysis based on the absence of fluorescence signal and light forward scatter (FSC) and side scatter (SSC) characteristics. 


\section{Cell-associated $\mathrm{Zn}^{2+}$}

Cell-associated $\mathrm{Zn}^{2+}$ was determined via inductively coupled plasma mass spectrometry (ICP-MS). Jurkat cells $\left(\begin{array}{llll}1 & \text { x } & 10^{5} & \text { cells } / \mathrm{ml})\end{array}\right)$ were treated with $\mathrm{nZnO}$ and incubated. At 4 and 24-hour time points, cells were washed twice in PBS, centrifuged at $500 \mathrm{x}$ g for $10 \mathrm{~min}$, re-suspended in FACS buffer, stained with $10 \mu \mathrm{L}$ of a FITC-labelled mouse anti-human HLA ABC antibody, and separated from any remaining extracellular nZnO via cell-sorting with a BD Influx cell sorter using Spigot software (version 6.1.4). Recovered live and dead intact cells were counted ( $>5$ times) using a hemocytometer and a Luna - II ${ }^{\mathrm{TM}}$ automated cell counter (Logos Biosystems; Annandale, VA). Sample aliquots were resuspended in a $10 \% \mathrm{HNO}_{3}$ (double distilled $15.8 \mathrm{~N}$ trace metal-grade) solution $(\mathrm{pH}$ 3.0) containing $5 \times 10^{5}$ cells and were mechanically lysed with a probe-tip sonicator at 50W for 2 minutes using 30 seconds on/10 second off pulse cycles. The acidified, lysed aliquots were stored in acid-rinsed eppendorf tubes overnight to ensure complete dissolution of any undissolved $\mathrm{nZnO}$. The lysed aliquots were then filtered using a $10 \mathrm{kDa}$ MW cut-off Eppendorf filter, Sartorius Vivaspin® 500 (Sartorius AG; Goettingen, Germany), to remove cellular debris prior to analysis. Control experiments were performed to demonstrate that $99 \%$ NP-derived zinc ions are recoverable using this methodology [34]. NP-only control samples demonstrated that the sorting procedure did not significantly affect the ionic zinc levels in the experimental samples. Quantitative analyses of the solutions were performed via ICP-MS.

\section{Dosimetry}

Two empirical methods, the Harvard Volume Centrifugation Method (VCM) [58, 59] and the in vitro sedimentation, diffusion and dosimetry (ISDD) computational model 
[60] were employed to determine the effective NP density of the agglomerates $\left(\rho_{e}, \mathrm{~g} / \mathrm{cm}^{3}\right)$ and the amount of nanomaterial deposited within a cell culture scenario, respectively. The effective volume of NP pellets $\left(V_{\text {pellet }}\right)$, was obtained by first creating a $\mathrm{nZnO}$ dispersion in cellular media as previously described [57]. The resulting dispersions were then centrifuged at $2,000 \times \mathrm{g}$ for one hour in TPP (Techno Plastic Products, Trasadingen, Switzerland) packed cell volume tubes and the resulting NP pellets were measured utilizing the TPP "easy read" measuring device by the same manufacturer. The $\rho_{e}$ of the NP agglomerates was calculated using the following equation (DeLoid et al.) [59].

$$
\rho_{e}=\rho_{\text {media }}+\left[\left(\frac{M_{Z n O}-M_{Z n O S o l}}{V_{\text {pellet }} S F}\right)\left(1-\frac{\rho_{\text {media }}}{\rho_{\text {Zno }}}\right)\right]
$$

The $\rho_{e}$ is determined as a function of the media density ( $\left.\rho_{\text {media }} \rho_{\text {media }}\right)$ and the material density of $\mathrm{ZnO}$ NPs $\left(\rho_{\mathrm{ZnO}}\right)$ of $5.606 \mathrm{~g} / \mathrm{cm}^{3}$. To account for the solubility of $\mathrm{ZnO}$, the mass of solubilized $\mathrm{ZnO}\left(M_{\mathrm{ZnOS} o l}\right)$ determined by ICP-MS (see "Extracellular Dissolution" section) was subtracted from the original mass of $\mathrm{ZnO}\left(\mathrm{M}_{\mathrm{ZnO}}\right)$. A theoretical stacking factor $(S F)$ of 0.634 was utilized to approximate random close stacking employed by many dosimetry models $[58,59]$. The ISDD model was provided by Justin G Teeguarden from the Pacific Northwest National Laboratory and is outlined in this paper [61].

\section{Zinc speciation determination}

The molecular speciation into amorphous $\mathrm{nZnO}$, zinc phosphate and zinc carbonate under cellular assay conditions was evaluated through FTIR, X-ray photoelectron spectroscopy (XPS) and TEM. For this assay, samples containing $32.4 \mu \mathrm{g} / \mathrm{mL}$ nanopure water-based $\mathrm{nZnO}$ stock solution were introduced to RPMI media and incubated. At 4 and 24 hours, the entire volume of one of the samples was centrifuged at $5000 \mathrm{x} \mathrm{g}$ for 30 
minutes to pellet any suspended $\mathrm{nZnO}$ or amorphous zinc complexes. The resulting pellet was rinsed with nanopure water, centrifuged and dried overnight at $60{ }^{\circ} \mathrm{C}$. FTIR pellets were made by combining $0.2 \mathrm{~g}$ of anhydrous $\mathrm{KBr}$ with $1.5 \mathrm{mg}$ of dried precipitate and then grinding into a fine powder. The powder was compressed with 8 tons of pressure for 4 minutes. Vibrational spectra were obtained with a Bruker Tensor 27 spectrometer and peak deconvolution performed using Opus 7.0.129 software. A Physics Electronics Versaprobe system outfitted with an $\mathrm{Al} \mathrm{K}_{\alpha} \mathrm{X}$-ray source delivering $25 \mathrm{~W}$ of power with a beam diameter of approximately $100 \mu \mathrm{m}$ was used to collect XPS spectra. Pass energy values of 117.5 eV and $23.65 \mathrm{eV}$ were used to obtain survey and high-resolution core level scans, respectively. Peak deconvolution and integrated peak analysis were performed with OriginLabPro 2017 software. TEM samples were obtained by dispersing a $\sim 0.5 \mathrm{mg}$ of sample in $\mathrm{EtOH}$ followed by sonication for 10 minutes and deposition onto carbon grids. Image collection was performed using a JEOL JEM-2100 HR analytical TEM.

\section{$\underline{\text { Reactive Oxygen Species (ROS) Detection }}$}

To assess cellular ROS levels, Jurkat cells ( 1 x $10^{5}$ cells/ml) were incubated with $\mathrm{nZnO}$ for 24 hours and then dually stained with $10 \mu \mathrm{L}$ of an anti-HLA-ABC PE antibody and $2.5 \mu \mathrm{M}$ of MitoSOX ${ }^{\mathrm{TM}}$ Red (Invitrogen; Eugene, OR). In the presence of mitochondrial superoxide, MitoSOX Red becomes oxidized and a fluorescent signal appears upon binding of the oxidized dye to nucleic acids [62]. For a positive control, cells were treated with $0.2 \mathrm{nM}$ antimycin-A to ensure that the cells were capable of producing ROS. Flow cytometry was used to measure mitochondrial superoxide levels with the percent positive MitoSOX cells designated as gated lymphocytes staining positive for both HLA-ABC and MitoSOX red. 


\section{$\underline{\text { Statistical analyses }}$}

Statistical analyses were performed using JMP Pro 12 software (SAS; Cary, NC). The cytotoxicity data were analysed using repeated measures of variance with post hoc comparisons and significance levels defined as $\mathrm{p}<0.05$ to determine statistical differences between the means and allow within-sample variation to be separated from betweensample variation. Data for Fig. 3.1-3.5 were analyzed using a two-way analysis of variance (ANOVA) to test for statistical significance of the model, and post hoc comparisons were used to test for statistically significant effects of treatment on the experimental outcome $(\mathrm{p}<0.05)$ with lowercase letters denoting statistical significance in the indicated figures. Linear least squares regression was employed to determine statistical significance between individual synthesis and control sample groups (Fig. 3.1-3.5). A linear regression was employed to determine the Pearson correlation coefficient $\left(\mathrm{R}^{2}\right)$ value for Fig. S3.3.

Principal component analysis (PCA) was used to reduce dimensionality of our multivariate data set to obtain canonical variables that are statistically independent of each other yet still representative of the experimental data [63]. Using the correlation method, PCA was performed with JMP Pro 12 software followed by common factor analysis using squared multiple correlation communality estimates and a varimax rotation method to obtain rotated principal components (PCs). A standard least squares regression model was used to evaluate the linearity of the measured $\mathrm{IC}_{50}$ values of the Jurkat cells against the obtained PCs to obtain a predictive model for NP-induced toxicity (Table S3.1 in the electronic supplementary information (ESI)). 


\section{Results}

$\underline{\text { X-ray Diffraction, XPS and TEM Analysis for Crystal Structure, Composition, and }}$ Morphology

A diverse panel of $\mathrm{nZnO}$ was synthesized by both wet chemical and high temperature flame spray hydrolysis. Four of the formulations (DEG, EtOH, EG and $\mathrm{NaOH}$ ) were produced using wet chemical forced hydrolysis synthesis (designated green in Table 3.1), while two nZnO samples, a spherical FSP (FSPS) and a rod-shaped FSP (FSPR), were prepared via flame spray pyrolysis (FSP; blue in Table 3.1). Finally, a nanosized silicacoated version of the FSPR sample ( $\mathrm{SiO}_{2}$-FSPR) and bulk $\mathrm{ZnO}$ (bulk) were employed as control samples (shown in dark grey in Table 3.1) given their reported tendencies to be biologically safer than most nanoscale $\mathrm{ZnO}$ formulations [21, 22]. Prior to the implementation of biological assays, all formulations were thoroughly characterized. The crystal structure, phase purity and compositional purity of the formulation panel were evaluated using XRD analysis and confirmed that all formulations were $\mathrm{nZnO}$ and displayed a wurtzite crystal phase without alternate crystal phases present (Fig. S3.1(a-b) in ESI) and XPS (Fig. S3.2). TEM (Fig. S3.1(c-j)) revealed that the nanoscale samples had average particle sizes in at least one dimension between $16-45 \mathrm{~nm}$. The bulk $\mathrm{ZnO}$ sample was found to contain very large particles (mostly $>500 \mathrm{~nm}$ ) of mixed morphology (Fig. S3.1j). TEM images showed that the $\mathrm{nZnO}$ samples were spherical in morphology except for the $\mathrm{NaOH}, \mathrm{FSPR}$ and $\mathrm{SiO}_{2}$-FSPR samples which exhibited rod-like morphology (Fig. S3.1(f, h \& i)). 


\section{$\underline{\text { Cellular toxicity and oxidative stress responses }}$}

To evaluate the toxicity and oxidative stress induced by the $\mathrm{nZnO}$ formulations, Jurkat leukemia T cells (Fig. 3.1(a)) and primary non-transformed CD4 ${ }^{+} \mathrm{T}$ cells (Fig. 3.1(b)) were treated with increasing concentrations of $\mathrm{nZnO}$ for 24 hours (see Table S3.1 for millimolar concentration values and therapeutic indices). The cytotoxic response of both cell types to the different NP formulations can be roughly divided into three groups Nanoparticles prepared with wet chemical methods (DEG, EG, EtOH and $\mathrm{NaOH}$ ) showed the greatest level of toxicity, followed by the FSP samples. Interestingly, the rod-shaped $\mathrm{nZnO}$ appeared less cytotoxic in both the wet chemical method group $(\mathrm{NaOH})$ and the FSP group (FSPR) than the spherical NPs synthesized in a similar fashion (EtOH and FSPS, respectively); however, these differences were more pronounced in the primary cells. Consistent with the literature, the $\mathrm{SiO}_{2}$-FSPR and bulk control samples were significantly less toxic than the $\mathrm{nZnO}$ formulations, whereas the positive ionic zinc $\mathrm{ZnSO}_{4}$ control exhibited the most toxicity. Additionally, the $\mathrm{IC}_{50}$ value obtained for $\mathrm{SiO}_{2}-\mathrm{FSPR}$ is in line with those values obtained for silica NP of comparable size indicating an efficient and hermetic coating $[64,65]$. Linear contrast analysis found the differences observed between three $\mathrm{ZnO}$ groupings, wet chemical, FSP and control samples to be statistically significant.

Intracellular ROS generation is considered a primary contributor to NP-induced toxicity in mammalian cells $[11,12,16,66]$. ROS produced by structural or functional damage to mitochondria can be assessed through the measurement of mitochondrial superoxide $[12,66,67]$. Figure 3.2 illustrates that the $\mathrm{nZnO}$ samples generated ROS in a formulation-dependent manner. Specifically, the most toxic formulation, DEG nZnO (Jurkat $\mathrm{IC}_{50}=20.7 \pm 3.1 \mu \mathrm{g} / \mathrm{mL}$ ), produced the highest percentage of ROS positive cells at 
$39.1 \pm 2.5 \%$, while treatment with the least toxic nanoscaled sample, FSPR $(86.4 \pm 4.7$ $\mu \mathrm{g} / \mathrm{mL})$, resulted in only $12.1 \pm 0.6 \%$ ROS producing cells. These findings directly correlated increases in NP-induced toxicity to corresponding increases in mitochondrial superoxide generation. The one exception, the $\mathrm{SiO}_{2}-\mathrm{FSPR} \mathrm{nZnO}$, produced only $6.1 \pm 0.2 \%$ of ROS positive cells despite showing significantly more toxicity than bulk $\mathrm{ZnO}$, possibly due to complexation or shielding of the electron donor or acceptor sites needed for mitochondrial damage $[23,68]$.

\section{$\underline{\text { Surface characterization and reactivity }}$}

As different synthesis methods are expected to result in unique surface chemistry, several characterizations were performed to elucidate these differences. Surface bound species and hydroxylation were identified using FTIR analysis. Present in all formulations was the characteristic broad Zn-O vibrational mode (Fig. 3.3) centered between $451 \mathrm{~cm}^{-1}$ and $475 \mathrm{~cm}^{-1}$, and the broad FTIR peak associated with surface adsorbed $\mathrm{OH}$ groups at $3410 \mathrm{~cm}^{-1}$ [69-72]. Notable differences present only in the wet chemically synthesized samples (Fig. 3.3(a)) included the O-H bending mode and the $v_{s}\left(\mathrm{COO}^{-}\right)$of carboxylic acid at $910 \mathrm{~cm}^{-1}$ at $1412 \mathrm{~cm}^{-1}$, respectively [71, 72]. Additionally, the DEG sample displayed a $v(\mathrm{C}-\mathrm{OH})$ mode at $1076 \mathrm{~cm}^{-1}$ and the $v_{a s}\left(\mathrm{COO}^{-}\right)$band at $1591 \mathrm{~cm}^{-1}$ [70-72]. The above mentioned peaks can be attributed to function group remnants of the synthesis precursor or the reaction solvent [25]. Finally, the $\mathrm{SiO}_{2}$ coating was confirmed through the presence of the Si-O-Si bending and Si-O-Si stretching modes found at 800 and $1081 \mathrm{~cm}^{-1}$, respectively [69].

The FTIR data confirmed that the NP formulations differed in the type and quantity of surface adsorbed functional groups and charged ions. These modifications, along with 
intrinsic surface defects are often conferred by the synthesis procedure, and alter the surface charge density and ionization potential of the nanocrystals resulting in the generation of an enhanced surface charge [25]. Zeta potential ( $\zeta$-potential) measurements (Table 3.1) verified these synthesis method-dependent differences. The positive $\zeta$-potential values of the uncoated samples were higher for samples with more surface-adsorbed functional groups and hydroxylation. When the $\mathrm{IC}_{50}$ in Jurkat cells was plotted as a function of $\zeta$-potential (Fig. S3.3 in ESI), a linear correlation $\left(\mathrm{R}^{2}=0.72 ; \mathrm{p}<0.0001\right)$ was observed for the uncoated $\mathrm{nZnO}$ samples. This correlation is interesting considering that all samples experienced similar electrophoretic mobility shifts when suspended in cellular media (Table 3.1). While this normalization of the NP surface charge in cellular media may be due in part to protein corona formation, these findings may also be skewed by free protein remaining in the media. Indeed, control experiments using a dried protein-coated DEG $\mathrm{nZnO}$ sample revealed a $\zeta$-potential measurement of $-11.8 \pm 0.39 \mathrm{mV}$ as compared to the value of $-6.80 \pm 0.85$ obtained for the wet sample [57]. This commonly observed surface charge normalization has prompted speculation that $\zeta$-potential values measured in aqueous solutions contribute little to NP-induced toxicity [23], however, our results clearly do not fit with this speculation. A possible explanation for this is intrinsic surface defects that contribute to the $\mathrm{nZnO}$ surface charge, may still be present in the interior of the $\mathrm{nZnO}$ crystals and contribute to their toxic response when internalized into cells. Additionally, the variably charged surfaces noted for the different formulations could result in differential attractive forces to organic and biological molecules present in the extracellular milieu and/or cellular environment. Indeed, Han et.al (2014), observed that differently charged $\mathrm{nZnO}$ experienced variable adsorption of humic acid at circumneutral $\mathrm{pH}$. In their 
study, a positively charged $\mathrm{nZnO}$ sample adsorbed higher amounts of the negatively charged humic acid along with concentration-dependent shifts in the measured zeta potential when compared to a negatively charged nZnO sample [73].

In addition to their ability to influence surface charge, intrinsic defects such as oxygen vacancies or interstitial $\mathrm{Zn}$ ions, increase the number of possible electron donor or receptor sites available to participate in abiotic redox reactions at the NP surface $[25,74$, 75]. Seen as an indirect measurement of redox potential, photocatalytic activity has been connected to synthesis modification and resulting biological outcomes [25]. The highly reactive valance band holes $\left(h_{v b}^{+}\right)$and conduction band electrons $\left(e_{c b}^{-}\right)$produced during $\mathrm{UV}$ irradiation participate in redox reactions at the NP surface to produce multiple ROS species, resulting in sulfo-rhodamine B fluorescent dye degradation [25, 74, 75]. Figure 3.3(c), demonstrates this time-dependent degradation of the dye for the EG formulation. The average catalytic rate constants $\left(k, \mathrm{~min}^{-1}\right)$ for the uncoated $\mathrm{nZnO}$ formulations varied from $0.076 \mathrm{~min}^{-1}$ (DEG) to $0.036 \mathrm{~min}^{-1}$ (FSPR) (Fig. 3.3(d)), while the $\mathrm{SiO}_{2}-\mathrm{FSPR}$ sample did not exhibit any significant photocatalytic activity. All reported $k$ values are consistent with current literature $[25,36,74]$. Additionally, rod-shaped NPs exhibited lower $k$ values compared to spherical $\mathrm{nZnO}$ samples demonstrating a strong dependence of $k$ values on NP shape [36]. The $k$ values for the spherical nZnO plus the bulk control, (Inset; Fig. 3.3(d)), demonstrated a positive correlation with the observed cytotoxicity for both the cell types, and is consistent with our previous findings [25]. $\mathrm{ZnSO}_{4}$ treatment did not result in any appreciable dye degradation with or without UV illumination thus eliminating ionic zinc as a contributor to the photocatalytic process. 


\section{Dissolution potential and zinc speciation}

$\mathrm{ZnO}$ NPs are known to release $\mathrm{Zn}^{2+}$ in many biologically relevant solutions [76] and induce cellular damage both extracellularly and intracellularly [13, 29-31]. To determine if a relationship existed between these two dissolution measures, extracellular (bars in Fig. 3.4) and intracellular free $\mathrm{Zn}^{2+}$ (line graph in Fig. 3.4) dissolution assays were performed concurrently. As seen in Fig. 3.4, a clear trend exists between extracellular zinc release and the measured intracellular free $\mathrm{Zn}^{2+}$ for all samples. Additionally, the wet chemically synthesized NPs exhibited higher extracellular and intracellular free $\mathrm{Zn}^{2+}$ levels than the FSP or control samples with two notable exceptions. First, the EtOH sample was less soluble than the FSP nZnO despite being a wet chemical formulation and having a larger specific surface area (SSA) of $67.1 \mathrm{~m}^{2} / \mathrm{g}$. However, the increased hydrodynamic size of this sample (Table 3.1) reduces its surface area-to-volume ratio thereby decreasing its dissolution potential. Secondly, the $\mathrm{SiO}_{2}$-FSPR released relatively low amounts of $\mathrm{Zn}^{2+}$, despite its small hydrodynamic size, due to the protective benefits of the silica coating [22]. The same general trend was apparent for all samples at both time points, with modest increases between 1.5 and $3.9 \%$ in the amount of $\mathrm{Zn}^{2+}$ released by 24 hours. Overall, these results agree with dissolution trends noted in the literature and are consistent with the kinetic profiles observed for these $\mathrm{nZnO}$ formulations in nanopure water (Fig. S3.4 in ESI).

One limitation of the extracellular dissolution and intracellular free $\mathrm{Zn}^{2+}$ assays discussed above is that they do not address zinc associated with cells as non-ionic complexed forms of $\mathrm{nZnO}[17,31,41,45]$. To address this, an assay was developed to isolate intact cells and subsequently measure the total cell associated zinc including nanomaterial attached to the outer membrane and all forms of intracellular zinc. Following 
$\mathrm{nZnO}$ treatment, Jurkat cells were isolated from unbound $\mathrm{nZnO}$ by FACS sorting, and equivalent numbers of cells mechanically lysed and chemically digested to ensure that any zinc associated with the cells would be in an ionic form. Following filtration to remove cellular debris, zinc concentrations were determined by ICP-MS. Additionally, control experiments verified that the cell sorting procedure did not capture any un-associated nanomaterial. At 4 hours, the cell-associated zinc varied with no significant trends apparent amongst the synthesis methods or particle morphology (Fig. 3.5). By 24 hours, a few patterns emerged. First, within the wet chemical synthesis group, the amount of cellassociated zinc follows the inverse trend observed for cytotoxicity. Specifically, the most toxic NPs (DEG) contained the least cell-associated zinc while the least toxic formulation in that group $(\mathrm{NaOH})$ had the most. This pattern was mirrored in $\mathrm{nZnO}$ samples created using high temperature conditions $\left(>1000{ }^{\circ} \mathrm{C}\right)$ including the FSPS, FSPR, $\mathrm{SiO}_{2}-\mathrm{FSPR}$ and bulk samples. Of interest are the observed inconsistencies between the intracellular free zinc data (Fig. 3.4) and cell-associated zinc experiments (Fig. 3.5). One might expect that NP formulations with higher amounts of intracellular free zinc would also contain higher amounts of cell-associated zinc; however, the opposite was found to be true.

It is evident from the extracellular, intracellular and cell-associated $\mathrm{Zn}^{2+}$ measurements, that there are other zinc species contributing to the cell-associated levels other than intracellular free zinc. Since $\mathrm{nZnO}$ readily aggregates into amorphous precipitates containing $\mathrm{nZnO}$, mixed phosphate/carbonate phases and serum proteins within the extracellular environment [77-79], it is probable that large amounts of zinc will attach to or enter cells in non-ionic zinc forms. To characterize the nature and composition of these forms, FTIR and XPS analysis was employed. FTIR analysis of agglomerates 
isolated from cellular media demonstrated the time-dependent evolution of these amorphous precipitates (Fig. S3.5 in ESI and Fig. 3.6(a)) through the disappearance of peaks associated with the surface adsorbed groups and the appearance of the amide I vibrational mode at $1651 \mathrm{~cm}^{-1}$ indicative of bound serum proteins or crystalline water [8082]. Furthermore, the development of zinc phosphate is evidenced by the broad band convolution of phosphate bending and stretching modes at $1031 \mathrm{~cm}^{-1}$ and phosphate shoulder peaks between 502 and $637 \mathrm{~cm}^{-1}[44,80,83,84]$, whereas complexed carbonate phases are evidenced by the $v_{\mathrm{s}}\left(\mathrm{CO}_{3}{ }^{2-}\right)$ anion and the $\mathrm{C}-\mathrm{O}$ stretching modes at $1405 \mathrm{~cm}^{-1}$ and $1535 \mathrm{~cm}^{-1}$, respectively $[43,85]$. XPS confirmed these compositional changes with the atomic additions of nitrogen and phosphorous and increases in the relative intensity of carbon and oxygen observed in the survey spectra (Fig. S3.7 in ESI). In addition, the single $\mathrm{Zn} 2 \mathrm{P}_{3 / 2}$ peak present at $1021.6 \mathrm{eV}$ in the as-prepared samples was replaced by two peaks due to a chemical shift consistent with a $\mathrm{Zn}-\mathrm{P}$ peak overlapping with the $\mathrm{Zn}-\mathrm{O}$ peak with an approximate difference of $+2-e V($ Fig. 3.7(d)).

To quantify the intensity variations observed between the different formulations, the ratio of crystalline $\mathrm{nZnO}$ to the amount of phosphate in the precipitates $\left(\mathrm{ZnO} / \mathrm{PO}_{4}{ }^{3-}\right)$ was determined by calculating the integrated area ratio of the $\mathrm{nZnO}$ peak to the broad phosphate band at $1031 \mathrm{~cm}^{-1}$ (Fig. 3.6). Since the broad peak from $\sim 350-700 \mathrm{~cm}^{-1}$ is no longer solely representative of pure $\mathrm{ZnO}$ vibrational modes, peak deconvolution was employed to separate the integrated area associated with phosphate species from that of pure $\mathrm{nZnO}$ vibrational modes (Fig. 3.6(b \& c), Fig. S3.6 and Table S3.3 in ESI). Interestingly, the area ratios (black spheres in Fig. 3.7(a \& b)) increased as the amount of cell-associated zinc increased and mirrored the geometric trend established with the cell- 
associated zinc levels. As corroboration, calculated Zn/P ratios obtained from XPS survey spectra atomic concentrations, (white triangles in Fig. 3.7(a \& b)) were generally consistent with the $\mathrm{ZnO} / \mathrm{PO}_{4}{ }^{3-}$ values obtained from the FTIR experiment. A notable exception was the $\mathrm{Zn} / \mathrm{P}$ ratio obtained for $\mathrm{SiO}_{2}-\mathrm{FSPR} \mathrm{nZnO}$ sample which was negligible in comparison to the $\mathrm{ZnO} / \mathrm{PO}_{4}{ }^{3-}$ ratio. Considering the slow dissolution kinetics of the $\mathrm{SiO}_{2}-\mathrm{FSPR} \mathrm{nZnO}$ in cellular media, any agglomerates formed will likely have high protein content with lower zinc phosphate/carbonate formation thus resulting in the observed zinc and phosphate values below the detection limit $(<0.1 \%)$.

Qualitative evidence of the FTIR and XPS results can be seen in the high resolution TEM images collected on the precipitates (Fig. S3.8 - Fig. S3.15 in ESI). Agglomerates of crystalline $\mathrm{nZnO}$, confirmed by the lattice fringes observed in the high-resolution images, can be seen embedded in an organic matrix within all the $\mathrm{nZnO}$ and control samples. Furthermore, the more soluble $\mathrm{nZnO}$ formulations (DEG and EG) contained smaller, more porous $\mathrm{nZnO}$ aggregates with larger amounts of visible amorphous material compared to the denser $\mathrm{ZnO}$ agglomerates observed in less soluble samples. Additionally, the FSPR and $\mathrm{SiO}_{2}-\mathrm{FSPR}$ formulations retained much of their rod-shaped morphology even up to 24 hours. Taken with the $\mathrm{ZnO} / \mathrm{PO}_{4}{ }^{3-}$ and $\mathrm{Zn} / \mathrm{P}$ calculations, these observations suggest that large increases in cell-associated zinc can be attributed to non-ionic zinc complexes.

\section{Dosimetry}

Increasing $\mathrm{nZnO}$ agglomerate size results in many downstream effects including decreased dissolution, increased sedimentation, and changes in the overall dosimetry of the NPs [86]. The in vitro sedimentation, diffusion and dosimetry (ISDD) computational model [61] was employed to determine the fraction of nanomaterial (in $\mu \mathrm{g}$ ) that is deposited 
onto an adherent cell layer for a given time frame. Figure 3.8(a) demonstrates that formulation-specific differences exist in the amount of $\mathrm{nZnO}$ deposited over 30 hours with differences likely due to stronger sedimentation effects exerted by $\mathrm{nZnO}$ with large hydrodynamic diameters (Table 3.1 and Fig. 3.8(b)). The deposition differences at 24 hours when plotted with the hydrodynamic diameters of the NPs illustrated this connection (Fig. 3.8(b)). It is noteworthy that the geometric trend observed for these measurements was nearly identical to that observed for the cell-associated trend (Fig, 3.5 and Fig. 3.7). The SSA measurements obtained by BET were also plotted in Fig $3.8 \mathrm{~b}$ to evaluate potential connection between dosimetry, hydrodynamic diameter and SSA. No clear patterns, however, were observed between these measures. While hydrodynamic diameter appears to correlate strongly to both dosimetry and cell-associated zinc in this study, there are cautions that need to be considered with the discussion of hydrodynamic size. One common artefact is the possible presence of very large agglomerates which may mask the detection of smaller agglomerates in the dispersion thus skewing the average hydrodynamic diameter. Additionally, agglomerates with a loosely packed open structure, allowing for exposed NP surface area within the agglomerates, may alter NP dissolution kinetics or mass transport in unpredictable ways. Despite these potential artefacts, hydrodynamic diameter appears to be a significant variable in NP-induced toxicity, but should be considered as part of a broader characterization protocol including BET, TEM and dosimetry measurements.

\section{Principal Component Analysis}

Given the highly correlated and complex nature of our multivariate data set, 2D principal component analysis (PCA) was used to determine if the individual formulations 
could be quantitatively separated based on their material properties. The $2 \mathrm{D}$ analysis identified two statistically independent principal components (PCs) that accounted for $74 \%$ of the observed experimental variability. From the table (Fig. 3.9(a)), the individual experimental factors appear to be grouped by their contribution to either oxidative stress responses $(\mathrm{PC} 1 ; 51 \%)$ or agglomeration potential (PC2; $23 \%)$. At first glance, the inclusion of photocatalysis with PC2 appears anomalous; however, increasing aggregation behavior decreases photon absorption leading to fewer electron-hole pairs and less ROS generation [87]. Qualitative groupings based on similar component scores (colored ellipses in Fig. 3.9(b)), revealed some interesting patterns. Not surprisingly, the bulk sample (purple ellipse) scored high for PC1 given its large hydrodynamic sizes and cell-associated zinc values. Whereas the $\mathrm{SiO}_{2}$-FSPR formulation (pink ellipse) associated negatively with both PCs due to decreased dissolution potential, ROS production, aggregation potential and photocatalytic activity. Amongst the uncoated nanosized samples, the most toxic samples, DEG and EG (yellow ellipse), scored positively for both PCs and is likely a consequence of high intracellular $\mathrm{Zn}^{2+}$ levels, stronger surface reactivity and faster photocatalytic activity. In contrast, the other samples (blue ellipse), with lower toxicity, demonstrate less surface reactivity thus lowering their oxidative stress contributions.

\section{Discussion}

This work sought to expand upon previous results demonstrating the importance of synthesis method in modulating surface reactivity, NP-induced toxicity and oxidative stress [25]. The goal of this research was two-fold: 1) to thoroughly characterize the surface reactivity, and dissolution potential of the $\mathrm{nZnO}$ panel and 2) identify the relative contributions of these material properties in NP-induced toxicity and oxidative stress. Upon 
assessing the toxicity of the $\mathrm{nZnO}$ panel against Jurkat leukemic and primary $\mathrm{CD}^{+}{ }^{+} \mathrm{T}$ cells, it was observed that NPs synthesized through wet chemical methods imparted greater toxicity to both cell types than those synthesized using FSP methods (Fig. 3.1). Several possible scenarios may explain these discrepancies.

First, the dissolution kinetics are remarkably different amongst the nZnO formulations. The more toxic samples demonstrate faster dissolution kinetics within the extracellular environment (Fig. 3.4). Consequently, these nZnO exhibit decreased agglomeration potential (Table 3.1) and increased intracellular zinc levels (Fig. 3.4). In comparison, as the toxicity of the $\mathrm{nZnO}$ formulations decreased, the kinetic balance shifted from extracellular dissolution towards more agglomerate formation and the cellular adherence of large amounts of non-ionic zinc as evidenced by the cell-associated zinc (Fig. 3.5 and 3.7) and dosimetry (Fig. 3.8) data. Ultimately, both extracellular and cellassociated zinc contribute to increased levels of intracellular free zinc and disruption of the zinc homeostasis of the cell $[13,28,29,66]$. However, identifying the source of intracellular free zinc levels can be difficult. One proposed mechanism is that large pools of extracellular ionic zinc that are liberated from the $\mathrm{nZnO}$ are taken up by the cell $[32,41$, 42]. This hypothesis is consistent with our extracellular/intracellular zinc data. While these results suggest that rapid increases in extracellular free zinc give rise to corresponding increases in intracellular free zinc, our experiments cannot definitively verify this correlation. Indeed, it is highly probable that some of the intracellular zinc levels observed may result from dissolution of non-ionic zinc intracellularly [17, 18, 28, 29, 31, 88], which would occur more rapidly for smaller $\mathrm{nZnO}$ agglomerates. Alternatively, studies have also shown that increased intracellular zinc in cells treated with highly toxic $\mathrm{nZnO}[13,28,89$ - 
91] results from disruption of cellular membranes by lipid peroxidation or by $\mathrm{nZnO}$ translocation into the cell [13, 28, 80-82].

Secondly, the variable synthesis methods resulted in differing surface reactivity. The hydrolysis based synthesis methods used here are known to produce NPs with multiple defects such as oxygen vacancies and interstitial zinc and oxygen atoms [92]. Additionally, singly and doubly ionized oxygen vacancies give rise to higher overall positive charge through increased $\cdot \mathrm{OH}$ surface absorption and excess $\mathrm{Zn}^{2+}[25,93]$. The presence of these synthesis method induced defects is validated through the high positive surface charge (Table 3.1 and Fig. S3.3) and faster photocatalytic rates (Fig. 3.3) observed for the wet chemical formulations and the direct correlation of this surface reactivity to the toxicity and ROS findings. (Fig. 3.1 and 3.2).

Dissolution kinetics and surface reactivity are often viewed as competing mechanisms for NP-induced toxicity. We contend, however, that these processes work concurrently as suggested by the principal component analysis. The PCA reduced the complexity of this data into two statistically independent principal components (PC1 and PC2) (Fig. 3.9). The first PC represents the material properties that are direct contributors of oxidative stress; specifically, increased $\zeta$-potentials, extracellular and intracellular dissolution, and cellular mitochondrial superoxide production. The second PC was more representative of agglomeration potential, with strong contributions coming from hydrodynamic size and cell-associated zinc. Regression analysis of the $\mathrm{IC}_{50}$ values for Jurkat cells as a linear function of PC1 and PC2 revealed that both PCs contribute significantly to the model ( $\mathrm{p}<0.05$; Table S3.2 in ESI). 
As the model suggests, PC1 (oxidative stress contributors) increases the NPinduced toxicity; while PC2 (agglomeration potential) works to decrease these effects. Additionally, PC score associations also appear to be related to the synthesis method employed, as two of our most toxic wet-chemically synthesized formulations (yellow ellipse in Fig. 3.9(b)), aligned strongly with PC1 when compared to less toxic samples. The following mechanistic scenario described below highlights the synergistic contribution from the two PCs. In the more toxic samples (higher PC1 vs PC2 scores), it is possible that the higher positive surface charges (aligned with PC1) for the wet chemical samples results in increased electrostatic interactions with both anionic lipids and protein domains, potentially leading to increased $\mathrm{nZnO}$ uptake and increases in the total intracellular zinc. Additionally, $\mathrm{nZnO}$ with intrinsic surface defects have higher ROS generating capability abiotically allowing for increased lipid peroxidation and passive cellular uptake. Once internalized, interactions between a more reactive formulation of $\mathrm{nZnO}$ and various cellular components would lead to heightened ROS generation and increased oxidation events within the cell. As for PC2 contributions, the smaller agglomerate sizes increase the extracellular dissolution potential and decrease non-ionic complex formation. A combination of zinc transporters and fluid phase pinocytosis pulling in larger amounts of free zinc, can elevate intracellular free zinc levels directly from liberated free zinc within the media [28-30,94]. This scenario demonstrates that while these PCs are statistically independent, they are not mutually exclusive. Even the most toxic of the $\mathrm{nZnO}$ formulations exhibited agglomeration and sedimentation potential and measurable cellassociated zinc (PC2), however those variables associated with PC1 were more influential in the toxic response. 


\section{Conclusions}

Identifying the physicochemical properties that most influence NP-induced toxicity has been a challenging endeavour in nanotoxicology. This investigation sought to resolve this question by evaluating a diverse panel of pristine $\mathrm{nZnO}$ samples. Through extensive analysis and mathematical modelling, two consistent sets of properties emerged as strong contributors to cytotoxicity. The first set of variables relating to surface reactivity, extracellular and intracellular $\mathrm{Zn}^{2+}$ dissolution, and oxidative stress responses has long been suggested as the primary mechanistic contributor to NP-induced toxicity. The second set (agglomeration tendencies, non-ionic zinc complexes and cell associated zinc levels) has also been implicated, but to a lesser degree. Collectively, our experimental results and the PCA model suggest that both sets of experimental variables contribute to $\mathrm{nZnO}$ cytotoxicity. Additionally, these findings suggest that the NP fabrication method employed results in differential material properties and resulting cellular responses. Wet chemically synthesized nanomaterials demonstrate increased surface reactivity and dissolution potential and decreased agglomeration potentially leading to the uptake of large amounts of non-ionic zinc complexes. Given that disruption of cellular zinc homeostasis and cell death will occur at a critical level of internalized zinc regardless of the NP formulation employed, other toxic mechanisms, such as surface reactivity, are likely responsible for the differential toxicity observed in our samples. This underscores the need for additional studies to evaluate how changes to specific NP physicochemical properties alter cellular uptake mechanisms, genotoxicity, apoptosis, and ROS-mediation pathways.

\section{Conflicts of interest}

There are no conflicts to declare. 


\section{Acknowledgements}

This research was supported in part by NSF-MRI awards (\#032233, \#0722699, \#0521315), NSF-RUI (DMR-0840227) and NIH (1R15CA141358-01). We also acknowledge support from the Biomolecular Research Center at Boise State University with funding from the NSF (\#0619793, \#0923535), the MJ Murdock Charitable Trust, and the Idaho State Board of Education. The authors thank Dr. Marion Lytle and the Biotrace Lab at Boise State University for the ICP-MS use and the members of the Advanced Materials Lab at Boise State University for the BET measurements. We gratefully acknowledge the use of CAMCOR TEM facilities at the University of Oregon, which have been purchased with a combination of federal and state funding. Additionally, we thank Dr. Georgios Sotiriou and Dr. Philip Demokritou at Harvard University for the generous donation of the FSPR and $\mathrm{SiO}_{2}$-FSPR FSP nanoparticle samples and Dr. Sukon Phanichphant at the Nanoscience Research Laboratory in Chiang Mai University, Chiang Mai, Thailand for the synthesis of the FSPS nanoparticles. 


\section{References}

1] Zvyagin, A. V.; Zhao, X.; Gierden, A.; Sanchez, W.; Ross, J. A.; Roberts, M. S. Imaging of zinc oxide nanoparticle penetration in human skin in vitro and in vivo. Journal of biomedical optics 2008, 13, 0640311-0640319.

2] Becheri, A.; Durr, M.; Lo Nostro, P.; Baglioni, P. Synthesis and characterization of zinc oxide nanoparticles: Application to textiles as uv-absorbers. Journal of Nanoparticle Research 2008, 10, 679-689.

3] Smijs, T. G. M.; Bouwstra, J. A. Focus on skin as a possible port of entry for solid nanoparticles and the toxicological impact. Journal of Biomedical Nanotechnology 2010, 6, 469-484.

4] Wehmas, L. C.; Anders, C.; Chess, J.; Punnoose, A.; Pereira, C. B.; Greenwood, J. A.; Tanguay, R. L. Comparative metal oxide nanoparticle toxicity using embryonic zebrafish. Toxicology reports 2015, 2, 702-715.

5] Xia, T.; Zhao, Y.; Sager, T.; George, S.; Pokhrel, S.; Li, N.; Schoenfeld, D.; Meng, H.; Lin, S.; Wang, X. et al. Decreased dissolution of zno by iron doping yields nanoparticles with reduced toxicity in the rodent lung and zebrafish embryos. ACS Nano 2011, 5, 1223-1235.

6] Espitia, P. J. P.; Soares, N. d. F. F.; dos Reis Coimbra, J. S.; de Andrade, N. J.; Cruz, R. S.; Medeiros, E. A. A. Zinc oxide nanoparticles: Synthesis, antimicrobial activity and food packaging applications. Food and Bioprocess Technology 2012, $5,1447-1464$.

7] Gunalan, S.; Sivaraj, R.; Rajendran, V. Green synthesized zno nanoparticles against bacterial and fungal pathogens. Progress in Natural Science: Materials International 2012, 22, 693-700.

8] Nair, S.; Sasidharan, A.; Rani, V. D.; Menon, D.; Nair, S.; Manzoor, K.; Raina, S. Role of size scale of zno nanoparticles and microparticles on toxicity toward bacteria and osteoblast cancer cells. Journal of Materials Science: Materials in Medicine 2009, 20, S235-S241.

9] Reddy, K. M.; Feris, K.; Bell, J.; Wingett, D. G.; Hanley, C.; Punnoose, A. Selective toxicity of zinc oxide nanoparticles to prokaryotic and eukaryotic systems. Applied Physics Letters 2007, 90, 1-8. 
10] Talebian, N.; Amininezhad, S. M.; Doudi, M. Controllable synthesis of zno nanoparticles and their morphology-dependent antibacterial and optical properties. Journal of Photochemistry and Photobiology B: Biology 2013, 120, 66-73.

11] Hanley, C.; Layne, J.; Punnoose, A.; Reddy, K. M.; Coombs, I.; Coombs, A.; Feris, K.; Wingett, D. Preferential killing of cancer cells and activated human $\mathrm{t}$ cells using zno nanoparticles. Nanotechnology 2008, 19, 1-10.

12] Hanley, C.; Thurber, A.; Hanna, C.; Punnoose, A.; Zhang, J. H.; Wingett, D. G. The influences of cell type and zno nanoparticle size on immune cell cytotoxicity and cytokine induction. Nanoscale Research Letters 2009, 4, 1409-1420.

13] Sharma, V.; Anderson, D.; Dhawan, A. Zinc oxide nanoparticles induce oxidative DNA damage and ros-triggered mitochondria mediated apoptosis in human liver cells (hepg2). Apoptosis 2012, 17, 852-870.

14] Bhattacharya, D.; Santra, C. R.; Ghosh, A. N.; Karmakar, P. Differential toxicity of rod and spherical zinc oxide nanoparticles on human peripheral blood mononuclear cells. Journal of Biomedical Nanotechnology 2014, 10, 707-716.

15] Gilbert, B.; Fakra, S. C.; Xia, T.; Pokhrel, S.; Madler, L.; Nel, A. E. The fate of zno nanoparticles administered to human bronchial epithelial cells. ACS Nano 2012, 6, 4921-4930.

16] Huang, C.-C.; Aronstam, R. S.; Chen, D.-R.; Huang, Y.-W. Oxidative stress, calcium homeostasis, and altered gene expression in human lung epithelial cells exposed to zno nanoparticles. Toxicology in Vitro 2010, 24, 45-55.

17] Mu, Q. S.; David, C. A.; Galceran, J.; Rey-Castro, C.; Krzeminski, L.; Wallace, R.; Bamiduro, F.; Milne, S. J.; Hondow, N. S.; Brydson, R. et al. Systematic investigation of the physicochemical factors that contribute to the toxicity of zno nanoparticles. Chemical Research in Toxicology 2014, 27, 558-567.

18] Muller, K. H.; Kulkarni, J.; Motskin, M.; Goode, A.; Winship, P.; Skepper, J. N.; Ryan, M. P.; Porter, A. E. Ph-dependent toxicity of high aspect ratio zno nanowires in macrophages due to intracellular dissolution. ACS Nano 2010, 4, 6767-6779.

19] Schaudien, D.; Knebel, J.; Creutzenberg, O. In vitro study revealed different size behavior of different nanoparticles. Journal of Nanoparticle Research 2012, 14, 19.

20] George, S.; Pokhrel, S.; Xia, T.; Gilbert, B.; Ji, Z. X.; Schowalter, M.; Rosenauer, A.; Damoiseaux, R.; Bradley, K. A.; Madler, L. et al. Use of a rapid cytotoxicity 
screening approach to engineer a safer zinc oxide nanoparticle through iron doping. ACS Nano 2010, 4, 15-29.

21] Gass, S.; Cohen, J. M.; Pyrgiotakis, G.; Sotiriou, G. A.; Pratsinis, S. E.; Demokritou, P. Safer formulation concept for flame-generated engineered nanomaterials. Acs Sustainable Chemistry \& Engineering 2013, 1, 843-857.

22] Sotiriou, G. A.; Watson, C.; Murdaugh, K. M.; Darrah, T. H.; Pyrgiotakis, G.; Elder, A.; Brain, J. D.; Demokritou, P. Engineering safer-by-design silica-coated zno nanorods with reduced DNA damage potential. Environmental Science-Nano 2014, $1,144-153$.

23] Yin, H.; Casey, P. S.; McCall, M. J.; Fenech, M. Effects of surface chemistry on cytotoxicity, genotoxicity, and the generation of reactive oxygen species induced by zno nanoparticles. Langmuir 2010, 26, 15399-408.

24] Park, S. J.; Park, Y. C.; Lee, S. W.; Jeong, M. S.; Yu, K.-N.; Jung, H.; Lee, J.-K.; Kim, J. S.; Cho, M.-H. Comparing the toxic mechanism of synthesized zinc oxide nanomaterials by physicochemical characterization and reactive oxygen species properties. Toxicology Letters 2011, 207, 197-203.

25] Punnoose, A.; Dodge, K.; Rasmussen, J. W.; Chess, J.; Wingett, D.; Anders, C. Cytotoxicity of zno nanoparticles can be tailored by modifying their surface structure: A green chemistry approach for safer nanomaterials. Acs Sustainable Chemistry \& Engineering 2014, 2, 1666-1673.

26] Bozym, R. A.; Chimienti, F.; Giblin, L. J.; Gross, G. W.; Korichneva, I.; Li, Y. A.; Libert, S.; Maret, W.; Parviz, M.; Frederickson, C. J. et al. Free zinc ions outside a narrow concentration range are toxic to a variety of cells in vitro. Experimental Biology and Medicine 2010, 235, 741-750.

27] Hsiao, I. L.; Huang, Y. J. Effects of various physicochemical characteristics on the toxicities of zno and tio2 nanoparticles toward human lung epithelial cells. Sci. Total Environ. 2011, 409, 1219-1228.

28] Kao, Y. Y.; Chen, Y. C.; Cheng, T. J.; Chiung, Y. M.; Liu, P. S. Zinc oxide nanoparticles interfere with zinc ion homeostasis to cause cytotoxicity. Toxicological Sciences 2012, 125, 462-472.

29] Othman, B. A.; Greenwood, C.; Abuelela, A. F.; Bharath, A. A.; Chen, S.; Theodorou, I.; Douglas, T.; Uchida, M.; Ryan, M.; Merzaban, J. S. et al. Correlative light-electron microscopy shows rgd-targeted zno nanoparticles dissolve in the intracellular environment of triple negative breast cancer cells and cause apoptosis 
with intratumor heterogeneity. Advanced Healthcare Materials 2016, 5, 13101325.

30] Sasidharan, A.; Chandran, P.; Menon, D.; Raman, S.; Nair, S.; Koyakutty, M. Rapid dissolution of zno nanocrystals in acidic cancer microenvironment leading to preferential apoptosis. Nanoscale 2011, 3, 3657-3669.

31] Shen, C. C.; James, S. A.; de Jonge, M. D.; Turney, T. W.; Wright, P. F. A.; Feltis, B. N. Relating cytotoxicity, zinc ions, and reactive oxygen in zno nanoparticleexposed human immune cells. Toxicological Sciences 2013, 136, 120130.

32] Song, W. H.; Zhang, J. Y.; Guo, J.; Zhang, J. H.; Ding, F.; Li, L. Y.; Sun, Z. T. Role of the dissolved zinc ion and reactive oxygen species in cytotoxicity of zno nanoparticles. Toxicology Letters 2010, 199, 389-397.

33] Yu, J.; Baek, M.; Chung, H. E.; Choi, S. J. Effects of physicochemical properties of zinc oxide nanoparticles on cellular uptake. Journal of Physics: Conference Series 2011, 304, 1-6.

34] Wingett, D.; Louka, P.; Anders, C. B.; Zhang, J. H.; Punnoose, A. A role of zno nanoparticle electrostatic properties in cancer cell cytotoxicity. Nanotechnology Science and Applications 2016, 9, 29-45.

35] Khan, M. F.; Ansari, A. H.; Hameedullah, M.; Ahmad, E.; Husain, F. M.; Zia, Q.; Baig, U.; Zaheer, M. R.; Alam, M. M.; Khan, A. M. et al. Sol-gel synthesis of thornlike zno nanoparticles endorsing mechanical stirring effect and their antimicrobial activities: Potential role as nano-antibiotics. Scientific Reports 2016, 6, 1-11.

36] McLaren, A.; Valdes-Solis, T.; Li, G. Q.; Tsang, S. C. Shape and size effects of zno nanocrystals on photocatalytic activity. Journal of the American Chemical Society 2009, 131, 12540-12541.

37] Becker, J.; Raghupathi, K. R.; St. Pierre, J.; Zhao, D.; Koodali, R. T. Tuning of the crystallite and particle sizes of zno nanocrystalline materials in solvothermal synthesis and their photocatalytic activity for dye degradation. The Journal of Physical Chemistry C 2011, 115, 13844-13850.

38] Jang, Y. J.; Simer, C.; Ohm, T. Comparison of zinc oxide nanoparticles and its nano-crystalline particles on the photocatalytic degradation of methylene blue. Materials Research Bulletin 2006, 41, 67-77. 
39] Li, D.; Haneda, H. Morphologies of zinc oxide particles and their effects on photocatalysis. Chemosphere 2003, 51, 129-137.

40] Li, M. H.; Pokhrel, S.; Jin, X.; Madler, L.; Damoiseaux, R.; Hoek, E. M. V. Stability, bioavailability, and bacterial toxicity of zno and iron-doped zno nanoparticles in aquatic media. Environmental Science \& Technology 2011, 45, 755-761.

41] Turney, T. W.; Duriska, M. B.; Jayaratne, V.; Elbaz, A.; O'Keefe, S. J.; Hastings, A. S.; Piva, T. J.; Wright, P. F. A.; Feltis, B. N. Formation of zinc-containing nanoparticles from $\mathrm{zn} 2+$ ions in cell culture media: Implications for the nanotoxicology of zno. Chemical Research in Toxicology 2012, 25, 2057-2066.

42] Amara, S.; Ben Slama, I.; Mrad, I.; Rihane, N.; Khemissi, W.; El Mir, L.; Ben Rhouma, K.; Abdelmelek, H.; Sakly, M. Effects of zinc oxide nanoparticles and/or zinc chloride on biochemical parameters and mineral levels in rat liver and kidney. Human \& Experimental Toxicology 2014, 33, 1150-1157.

43] Wahab, R.; Ansari, S. G.; Kim, Y. S.; Dar, M. A.; Shin, H. S. Synthesis and characterization of hydrozincite and its conversion into zinc oxide nanoparticles. Journal of Alloys and Compounds 2008, 461, 66-71.

44] Jung, S. H.; Oh, E.; Shim, D.; Park, D. H.; Cho, S.; Lee, B. R.; Jeong, Y. U.; Lee, K. H.; Jeong, S. H. Sonochemical synthesis of amorphous zinc phosphate nanospheres. Bull. Korean Chem. Soc. 2009, 30, 2280-2282.

45] Lv, J.; Zhang, S.; Luo, L.; Han, W.; Zhang, J.; Yang, K.; Christie, P. Dissolution and microstructural transformation of zno nanoparticles under the influence of phosphate. Environmental Science \& Technology 2012, 46, 7215-7221.

46] Demokritou, P.; Buchel, R.; Molina, R. M.; Deloid, G. M.; Brain, J. D.; Pratsinis, S. E. Development and characterization of a versatile engineered nanomaterial generation system (venges) suitable for toxicological studies. Inhalation Toxicology 2010, 22, 107-116.

47] Mueller, R.; Madler, L.; Pratsinis, S. E. Nanoparticle synthesis at high production rates by flame spray pyrolysis. Chemical Engineering Science 2003, 58, 19691976.

48] Pratsinis, S. E. Flame aerosol synthesis of ceramic powders. Progress in Energy and Combustion Science 1998, 24, 197-219. 
49] Pratsinis, S. E. Aerosol-based technologies in nanoscale manufacturing: From functional materials to devices through core chemical engineering. Aiche Journal 2010, 56, 3028-3035.

50] Pratsinis, S. E.; Vemury, S. Particle formation in gases: A review. Powder Technology 1996, 88, 267-273.

51] Wegner, K.; Pratsinis, S. E. Scale-up of nanoparticle synthesis in diffusion flame reactors. Chemical Engineering Science 2003, 58, 4581-4589.

52] Wu, C.; Qiao, X. H.; Chen, J. G.; Wang, H. S.; Tan, F. T.; Li, S. T. A novel chemical route to prepare zno nanoparticles. Materials Letters 2006, 60, 1828-1832.

53] Liewhiran, C.; Phanichphant, S. Influence of thickness on ethanol sensing characteristics of doctor-bladed thick film from flame-made zno nanoparticles. Sensors 2007, 7, 185-201.

54] Lutterotti, L.; Scardi, P.; Maistrelli, P. Ls1 - a computer-program for simultaneous refinement of material structure and microstructure. Journal of Applied Crystallography 1992, 25, 459-462.

55] Schneider, C. A.; Rasband, W. S.; Eliceiri, K. W. Nih image to imagej: 25 years of image analysis. Nature Methods 2012, 9, 671-675.

56] Taurozzi, J. S.; Hackley, V. A.; Wiesner, M. R. Ultrasonic dispersion of nanoparticles for environmental, health and safety assessment - issues and recommendations. Nanotoxicology 2011, 5, 711-729.

57] Anders, C. B.; Chess, J. J.; Wingett, D. G.; Punnoose, A. Serum proteins enhance dispersion stability and influence the cytotoxicity and dosimetry of zno nanoparticles in suspension and adherent cancer cell models. Nanoscale Research Letters 2015, 10, 1-22.

58] Cohen, J. M.; Teeguarden, J. G.; Demokritou, P. An integrated approach for the in vitro dosimetry of engineered nanomaterials. Part. Fibre Toxicol. 2014, 11.

59] DeLoid, G.; Cohen, J. M.; Darrah, T.; Derk, R.; Rojanasakul, L.; Pyrgiotakis, G.; Wohlleben, W.; Demokritou, P. Estimating the effective density of engineered nanomaterials for in vitro dosimetry. Nature Communications 2014, 5.

60] Hinderliter, P. M.; Minard, K. R.; Orr, G.; Chrisler, W. B.; Thrall, B. D.; Pounds, J. G.; Teeguarden, J. G. Isdd: A computational model of particle sedimentation, 
diffusion and target cell dosimetry for in vitro toxicity studies. Part. Fibre Toxicol. 2010, 7.

61] Hinderliter, P. M.; Minard, K. R.; Orr, G.; Chrisler, W. B.; Thrall, B. D.; Pounds, J. G.; Teeguarden, J. G. Isdd: A computational model of particle sedimentation, diffusion and target cell dosimetry for in vitro toxicity studies. Particle and Fibre Toxicology 2010, 7, 1-20.

62] Mukhopadhyay, P.; Rajesh, M.; Haskó, G.; Hawkins, B. J.; Madesh, M.; Pacher, P. Simultaneous detection of apoptosis and mitochondrial superoxide production in live cells by flow cytometry and confocal microscopy. Nature protocols $\mathbf{2 0 0 7 , 2 ,}$ 2295-2301.

63] Wang, X. Z.; Yang, Y.; Li, R. F.; McGuinnes, C.; Adamson, J.; Megson, I. L.; Donaldson, K. Principal component and causal analysis of structural and acute in vitro toxicity data for nanoparticles. Nanotoxicology 2014, 8, 465-476.

64] Napierska, D.; Thomassen, L. C. J.; Rabolli, V.; Lison, D.; Gonzalez, L.; KirschVolders, M.; Martens, J. A.; Hoet, P. H. Size-dependent cytotoxicity of monodisperse silica nanoparticles in human endothelial cells. Small 2009, 5, 846853.

65] Malugin, A.; Herd, H.; Ghandehari, H. Differential toxicity of amorphous silica nanoparticles toward phagocytic and epithelial cells. Journal of Nanoparticle Research 2011, 13, 5381-5396.

66] Xia, T.; Kovochich, M.; Liong, M.; Mädler, L.; Gilbert, B.; Shi, H.; Yeh, J.; Zink, J.; Nel, A. Comparison of the mechanism of toxicity of zinc oxide and cerium oxide nanoparticles based on dissolution and oxidative stress properties. ACS Nano $\mathbf{2 0 0 8 ,}$ 2, 2121-2134.

67] Rasmussen, J. W.; Martinez, E.; Louka, P.; Wingettt, D. G. Zinc oxide nanoparticles for selective destruction of tumor cells and potential for drug delivery applications. Expert Opinion on Drug Delivery 2010, 7, 1063-1077.

68] Ramasamy, M.; Das, M.; An, S. S. A.; Yi, D. K. Role of surface modification in zinc oxide nanoparticles and its toxicity assessment toward human dermal fibroblast cells. International Journal of Nanomedicine 2014, 9, 3707-3718.

69] Xia, H.-L.; Tang, F.-Q. Surface synthesis of zinc oxide nanoparticles on silica spheres: Preparation and characterization. The Journal of Physical Chemistry B 2003, 107, 9175-9178. 
70] Farbun, I. A.; Romanova, I. V.; Terikovskaya, T. E.; Dzanashvili, D. I.; Kirillov, S. A. Complex formation in the course of synthesis of zinc oxide from citrate solutions. Russian Journal of Applied Chemistry 2007, 80, 1798-1803.

71] Max, J. J.; Chapados, C. Infrared spectroscopy of aqueous carboxylic acids: Malic acid. Journal of Physical Chemistry A 2002, 106, 6452-6461.

72] Max, J. J.; Chapados, C. Infrared spectroscopy of aqueous carboxylic acids: Comparison between different acids and their salts. Journal of Physical Chemistry A 2004, 108, 3324-3337.

73] Han, Y.; Kim, D.; Hwang, G.; Lee, B.; Eom, I.; Kim, P. J.; Tong, M. P.; Kim, H. Aggregation and dissolution of zno nanoparticles synthesized by different methods: Influence of ionic strength and humic acid. Colloids and Surfaces aPhysicochemical and Engineering Aspects 2014, 451, 7-15.

74] Gupta, J.; Barick, K. C.; Bahadur, D. Defect mediated photocatalytic activity in shape-controlled zno nanostructures. Journal of Alloys and Compounds 2011, 509, 6725-6730.

75] Saliani, M.; Jalal, R.; Goharshadi, E. K. Mechanism of oxidative stress involved in the toxicity of zno nanoparticles against eukaryotic cells. Nanomedicine Journal 2016, 3, 1-14.

76] Eixenberger, J.; Anders, C.; Hermann, R.; Brown, R.; Reddy, K. M.; Punnoose, A.; Wingett, D. Rapid dissolution of zno nanoparticles induced by biological buffers significantly impacts cytotoxicity. Chemical Research in Toxicology 2017, 30, 1641-1651.

77] Lesniak, A.; Fenaroli, F.; Monopoli, M. R.; Aberg, C.; Dawson, K. A.; Salvati, A. Effects of the presence or absence of a protein corona on silica nanoparticle uptake and impact on cells. ACS Nano 2012, 6, 5845-5857.

78] Maiorano, G.; Sabella, S.; Sorce, B.; Brunetti, V.; Malvindi, M. A.; Cingolani, R.; Pompa, P. P. Effects of cell culture media on the dynamic formation of proteinnanoparticle complexes and influence on the cellular response. ACS Nano 2010, 4, 7481-7491.

79] Monopoli, M. P.; Aberg, C.; Salvati, A.; Dawson, K. A. Biomolecular coronas provide the biological identity of nanosized materials. Nature Nanotechnology 2012, 7, 779-786. 
80] Yuan, A. Q.; Liao, S.; Tong, Z. F.; Wu, J.; Huang, Z. Y. Synthesis of nanoparticle zinc phosphate dihydrate by solid state reaction at room temperature and its thermochemical study. Materials Letters 2006, 60, 2110-2114.

81] Wang, J. D.; Li, D.; Liu, J. K.; Yang, X. H.; He, J. L.; Lu, Y. One-step preparation and characterization of zinc phosphate nanocrystals with modified surface. Soft Nanoscience Letters 2011, Vol.01No.03, 81-85

82] Barth, A. Infrared spectroscopy of proteins. Biochimica Et Biophysica ActaBioenergetics 2007, 1767, 1073-1101.

83] Wang, Y.; Wöll, C. Chemical reactions on metal oxide surfaces investigated by vibrational spectroscopy. Surface Science 2009, 603, 1589-1599.

84] Pawlig, O.; Trettin, R. Synthesis and characterization of alpha-hopeite, zn-3(po4)(2)center dot 4h(2)o. Materials Research Bulletin 1999, 34, 1959-1966.

85] Hales, M. C.; Frost, R. L. Synthesis and vibrational spectroscopic characterisation of synthetic hydrozincite and smithsonite. Polyhedron 2007, 26, 4955-4962.

86] David, C. A.; Galceran, J.; Rey-Castro, C.; Puy, J.; Companys, E.; Salvador, J.; Monné, J.; Wallace, R.; Vakourov, A. Dissolution kinetics and solubility of zno nanoparticles followed by agnes. The Journal of Physical Chemistry C 2012, 116, 11758-11767.

87] Jassby, D.; Farner Budarz, J.; Wiesner, M. Impact of aggregate size and structure on the photocatalytic properties of tio2 and zno nanoparticles. Environ Sci Technol 2012, 46, 6934-6941.

88] Wang, B.; Zhang, Y. Y.; Mao, Z. W.; Yu, D. H.; Gao, C. Y. Toxicity of zno nanoparticles to macrophages due to cell uptake and intracellular release of zinc ions. Journal of nanoscience and nanotechnology 2014, 14, 5688-5696.

89] Lin, J. Q.; Zhang, H. W.; Chen, Z.; Zheng, Y. G. Penetration of lipid membranes by gold nanoparticles: Insights into cellular uptake, cytotoxicity, and their relationship. ACS Nano 2010, 4, 5421-5429.

90] Moghadam, B. Y.; Hou, W. C.; Corredor, C.; Westerhoff, P.; Posner, J. D. Role of nanoparticle surface functionality in the disruption of model cell membranes. Langmuir 2012, 28, 16318-16326. 
91] Yang, H.; Liu, C.; Yang, D.; Zhang, H.; Xi, Z. Comparative study of cytotoxicity, oxidative stress and genotoxicity induced by four typical nanomaterials: The role of particle size, shape and composition. J. Appl. Toxicol. 2009, 29, 69-78.

92] Zheng, Y.; Chen, C.; Zhan, Y.; Lin, X.; Zheng, Q.; Wei, K.; Zhu, J.; Zhu, Y. Luminescence and photocatalytic activity of zno nanocrystals: Correlation between structure and property. Inorganic Chemistry 2007, 46, 6675-6682.

93] Yang, Q. B.; Lin, T. S.; Burton, C.; Park, S. H.; Ma, Y. F. Physicochemical insights of irradiation-enhanced hydroxyl radical generation from zno nanoparticles. Toxicology Research 2016, 5, 482-491.

94] Condello, M.; De Berardis, B.; Ammendolia, M. G.; Barone, F.; Condello, G.; Degan, P.; Meschini, S. Zno nanoparticle tracking from uptake to genotoxic damage in human colon carcinoma cells. Toxicology in Vitro 2016, 35, 169-179. 
Tables and Figures

\section{nZnO-induced Toxicity}

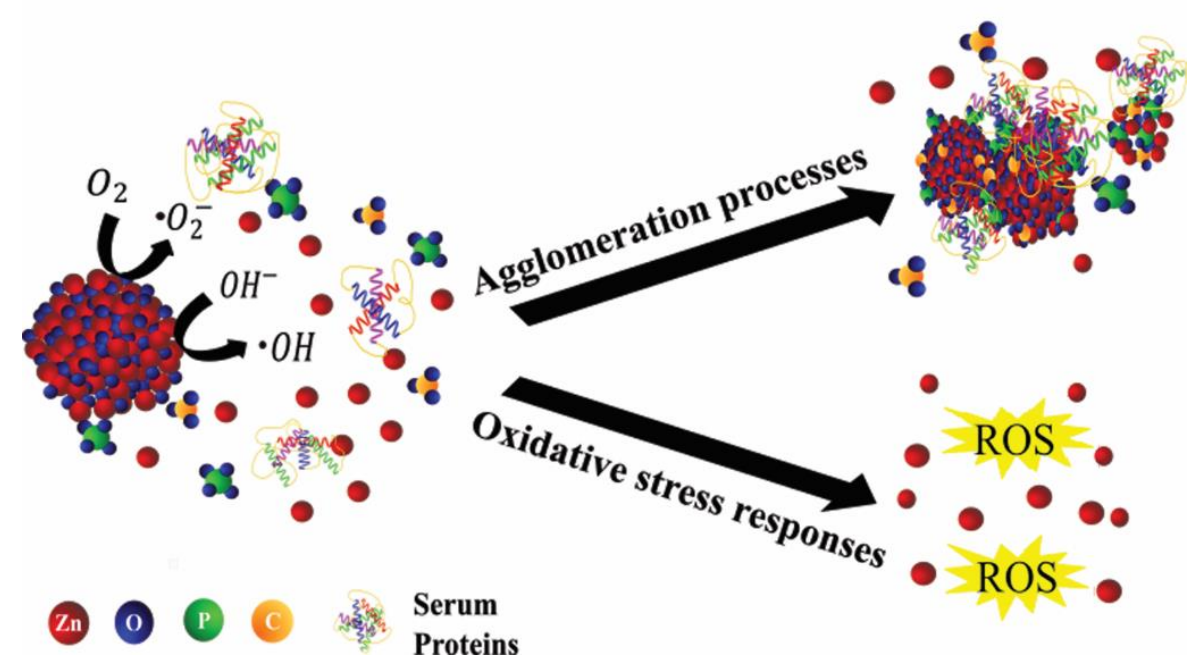

TOC 3.1 nZnO synthesis approach results in unique surface chemistries which influence agglomeration tendencies, dissolution potential, oxidative stress responses and NP-induced toxicity. 


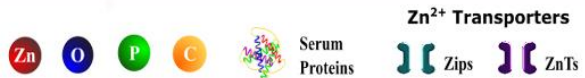

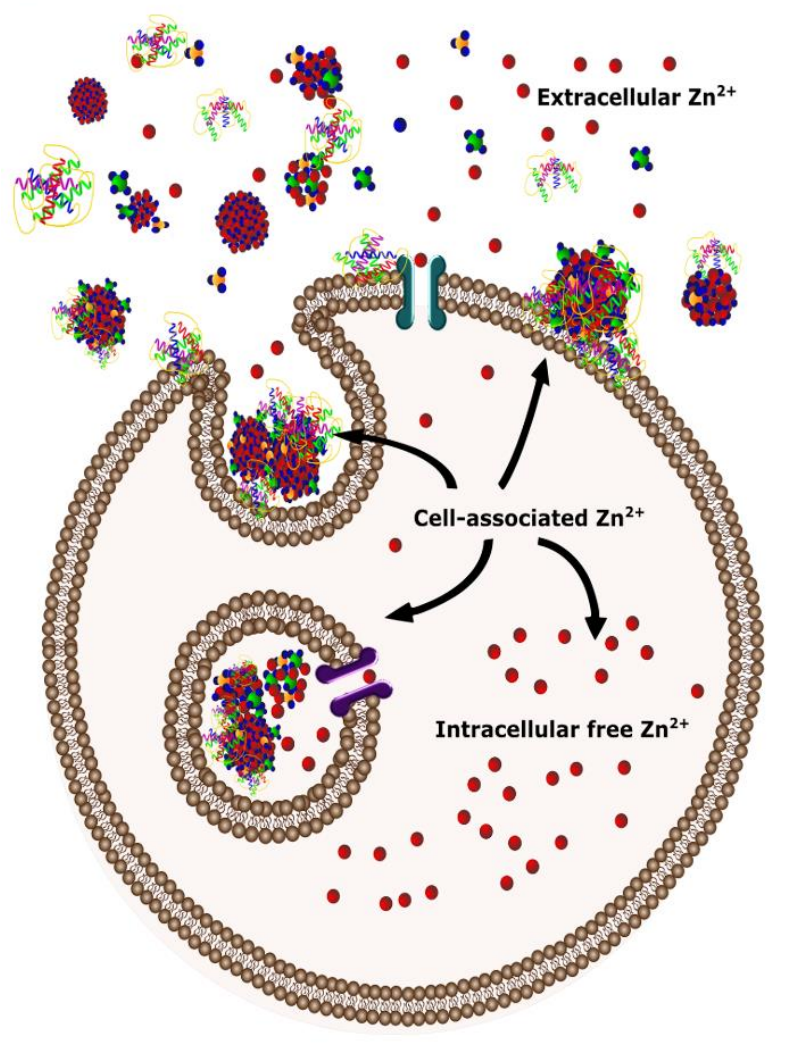

Schematic 3.1

Illustration depicting the $\mathrm{nZnO}$ dissolution processes and interactions with cells after NP treatment. 
Table 3.1 Physical properties of $\mathrm{ZnO}$ formulations measured via TEM, BET, UV-Vis, and DLS. Wet chemically and FSP synthesized formulations are shaded green and blue, respectively, while the control samples are indicated with the dark grey. The average size values are based on a minimum of 100 measured NPs on TEM images. The hydrodynamic diameters were determined by measuring the number of particles within each size bin in the histogram distribution.

\begin{tabular}{|c|c|c|c|c|c|}
\hline \multirow{2}{*}{$\begin{array}{c}\text { Formulation } \\
\text { (Band Gap; eV) }\end{array}$} & \multirow{2}{*}{$\begin{array}{l}\text { Average } \\
\text { Size } \\
(\mathrm{nm})\end{array}$} & \multirow{2}{*}{$\begin{array}{c}\text { Specific } \\
\text { Surface } \\
\text { Area }\left(\mathrm{m}^{2} / \mathrm{g}\right)\end{array}$} & \multicolumn{2}{|c|}{$\zeta$-Potential $(\mathrm{mV})^{\mathrm{a}}$} & \multirow{2}{*}{$\begin{array}{l}\text { Hydrodynamic } \\
\text { Diameter } \\
\text { (Media) } \\
\text { (nm) }\end{array}$} \\
\hline & & & Water & $\begin{array}{r}\text { Media (4 h) } \\
(24 \mathrm{~h})\end{array}$ & \\
\hline $\begin{array}{l}\text { DEG } \\
(3.36)\end{array}$ & $33 \pm 7$ & 34.5 & $29.7 \pm 0.19$ & $\begin{array}{l}-5.71 \pm 0.49 \\
-6.80 \pm 0.85\end{array}$ & $221.7 \pm 14.16$ \\
\hline $\begin{array}{c}\text { EG } \\
(3.36)\end{array}$ & $29 \pm 5$ & 22.5 & $27.6 \pm 0.37$ & $\begin{array}{l}-5.58 \pm 0.85 \\
-6.68 \pm 0.46\end{array}$ & $544.9 \pm 120$ \\
\hline $\begin{array}{l}\text { EtOH } \\
(3.36)\end{array}$ & $16 \pm 2$ & 67.1 & $23.0 \pm 0.25$ & $\begin{array}{l}-5.86 \pm 0.90 \\
-6.69 \pm 0.48\end{array}$ & $630.4 \pm 32.5$ \\
\hline $\begin{array}{l}\mathrm{NaOH} \\
(3.31)\end{array}$ & $\begin{array}{l}\mathrm{L}: 79 \pm 25 \\
\mathrm{~W}: 21 \pm 5\end{array}$ & 38 & $18.9 \pm 0.18$ & $\begin{array}{l}-6.28 \pm 0.34 \\
-6.89 \pm 0.11\end{array}$ & $623.2 \pm 128$ \\
\hline $\begin{array}{l}\text { FSPS } \\
(3.38)\end{array}$ & $43 \pm 10$ & 25 & $18.9 \pm 0.18$ & $\begin{array}{l}-6.11 \pm 0.72 \\
-6.53 \pm 0.65\end{array}$ & $227.2 \pm 15.6$ \\
\hline $\begin{array}{l}\text { FSPR } \\
(3.38)\end{array}$ & $\begin{array}{l}\mathrm{L}: 94 \pm 24 \\
\mathrm{~W}: 26 \pm 5\end{array}$ & 41.0 & $17.8 \pm 0.12$ & $\begin{array}{l}-6.29 \pm 066 \\
-6.36 \pm 0.70\end{array}$ & $669.7 \pm 119$ \\
\hline $\begin{array}{c}\mathrm{SiO}_{2}-\mathrm{FSPR} \\
(3.39)\end{array}$ & 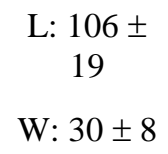 & 55.0 & $-44.1 \pm 0.23$ & $\begin{array}{l}-1.45 \pm 0.46 \\
-6.18 \pm 0.50\end{array}$ & $148.0 \pm 1.26$ \\
\hline $\begin{array}{l}\text { Bulk } \\
(3.39)\end{array}$ & $852 \pm 350$ & 1.25 & $7.23 \pm 2.84$ & $\begin{array}{l}-6.67 \pm 0.39 \\
-6.30 \pm 0.23\end{array}$ & $2039.5 \pm 137.7$ \\
\hline
\end{tabular}

${ }^{\mathrm{a}} \mathrm{The} \mathrm{pH}$ values of the $\mathrm{nZnO}$ dispersions varied from $7.5-7.7$ for all dispersions used for $\zeta$-Potential measurements. 
(a)

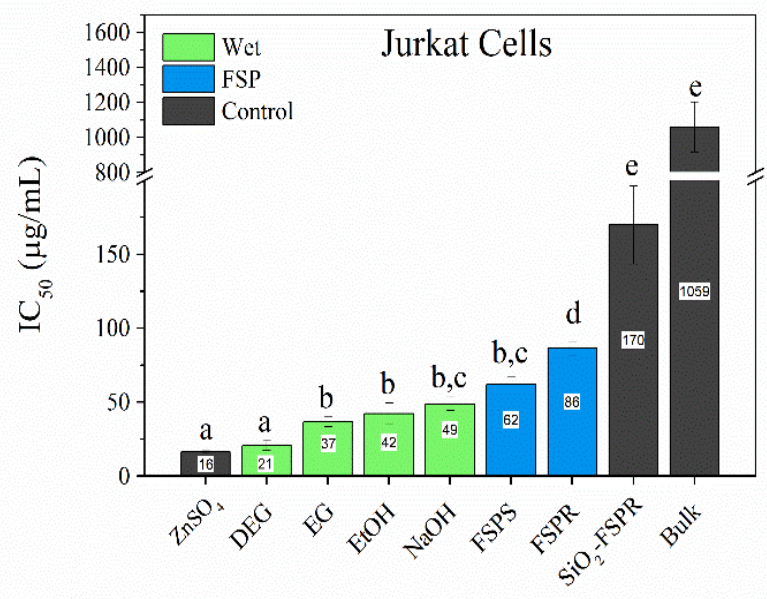

nZnO Formulation (b)

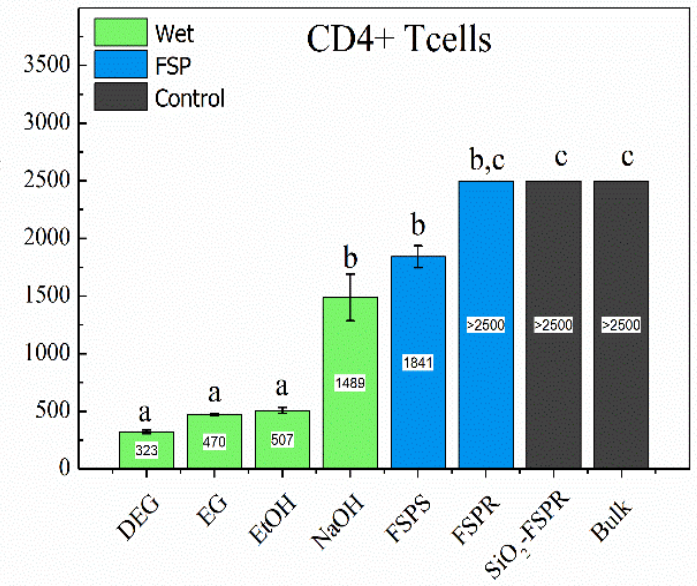

nZnO Formulation

Figure 3.1 NP-induced toxicity values for (a) Jurkat leukemic cell and (b) normal primary $\mathrm{CD}^{+} \mathrm{T}$ cell viability at 24 hours after treatment with the wet chemical method (green bars; wet), flame spray pyrolysis (blue bars; FSP) nZnO formulations and the $\mathrm{SiO}_{2}$-FSPR and bulk controls (black bars; control). The white labels on the histogram bars depict the IC50values obtained for the indicated sample. The histogram bars were ordered from lowest to highest $\mathrm{IC}_{50}$ for both cell types to depict the synthesis method trends observed for the NP-induced toxicity. Cultures were treated concurrently with varying concentrations of $\mathrm{nZnO}$ dispersed in nanopure water/RPMI for 24 hours and cell viability was evaluated (means \pm standard error, minimum of $n=3$ ) using Alamar blue staining (Jurkat Cells) or flow cytometry with PI staining $\left(\mathrm{CD4}^{+} \mathrm{T}\right.$ cells). Statistical analysis was performed using repeated measures analysis of variance and model-based means post hoc test $(p<0.05)$ with differing letters denoting statistical significance. Linear contrast models were used to determine statistical significance between the wet chemical, flame spray pyrolysis and control samples. 


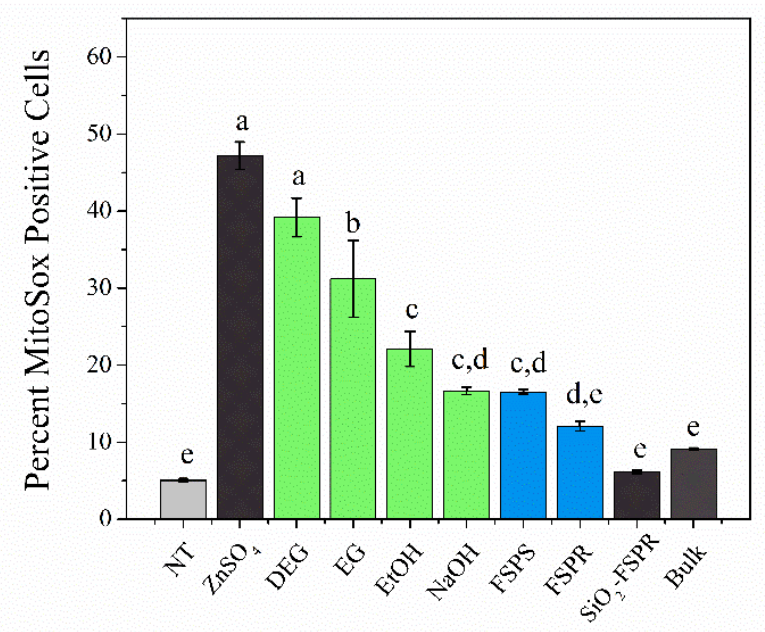

Formulations

Figure 3.2 nZnO samples generated ROS in a formulation-dependent manner. Mitochondrial superoxide generation by all $\mathrm{nZnO}$ at 24-hour post treatment with 32.4 $\mu \mathrm{g} / \mathrm{mL} \mathrm{ZnO}$ using flow cytometry and MitoSox ${ }^{\mathrm{TM}}$ Red staining. Statistical analysis was performed using repeated measures analysis of variance and model-based means post hoc test $(p<0.05)$ with differing letters denoting statistical significance. 
(a)

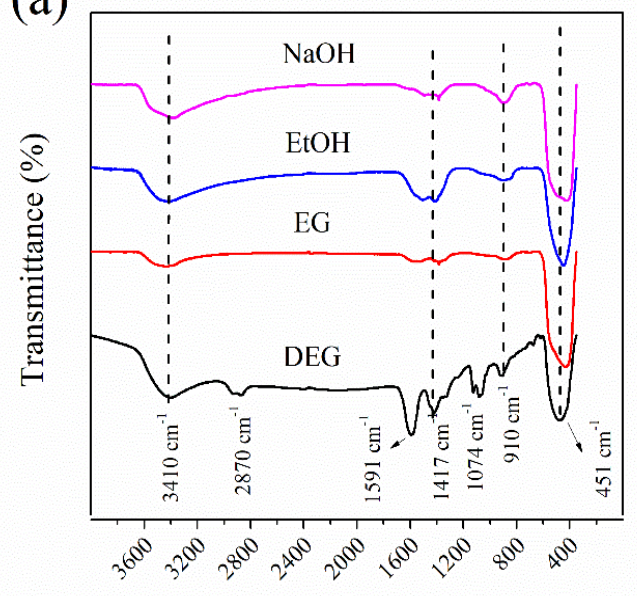

Wavenumber $\left(\mathrm{cm}^{-1}\right)$

(c)

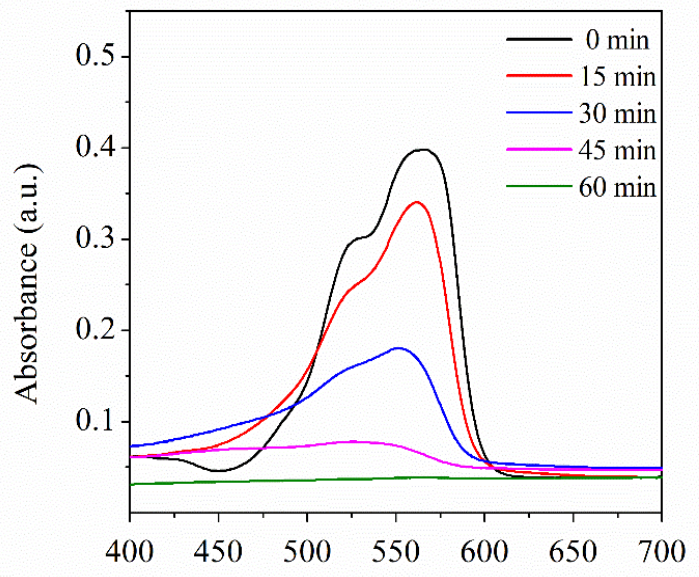

Wavelength (nm) (b)

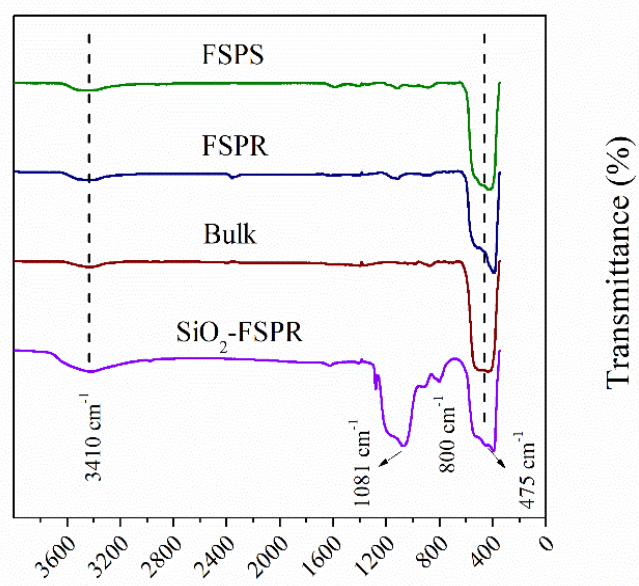

Wavenumber $\left(\mathrm{cm}^{-1}\right)$

(d)

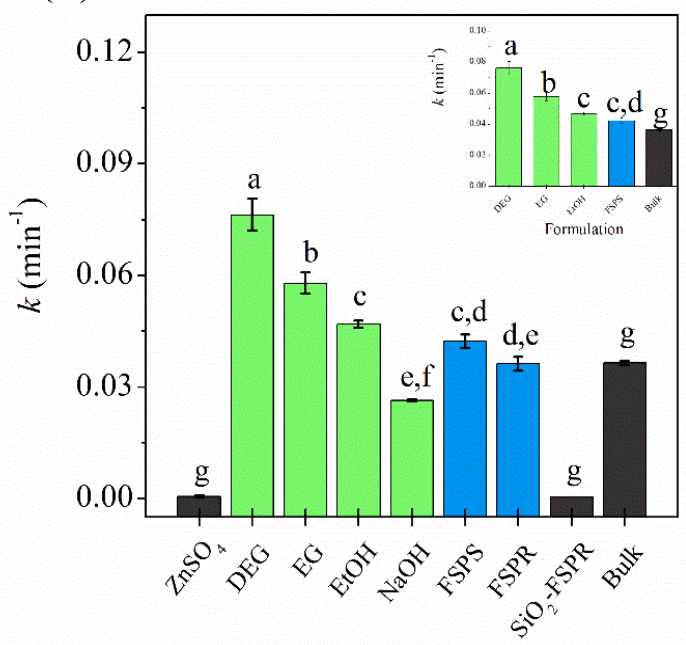

Formulation

Figure 3.3 Surface property characterization for the powered samples ((a) and (b)). and catalytic activity plots ((c) and (d)) depicting the UV/Vis monitored fluorescence of the model sulfo-Rhodamine $B$ dye and the average dye degradation kinetic values obtained for the evaluated treatment conditions. FTIR spectra for (a) wet chemical synthesis methods, and (b) heat treatment methods illustrate the peaks observed and the corresponding wavenumber values. The graph in (c) is representative of the time-dependent plots obtained and demonstrate the photocatalytic decomposition of sulfo-Rhodamine $B$ dye in nanopure water after treatment with EG NPs. The histogram in (d) depicts the average catalytic rate constants $\left(\mathrm{k}, \mathrm{min}^{-1}\right)$ for the $\mathrm{nZnO}$ and control samples. Statistical analysis in (d) was performed using repeated measures analysis of variance and model-based means post hoc test $(p<0.05)$ with differing letters denoting statistical significance. Histogram bars represent $n=3$ replicates with error bars indicating s.e. 


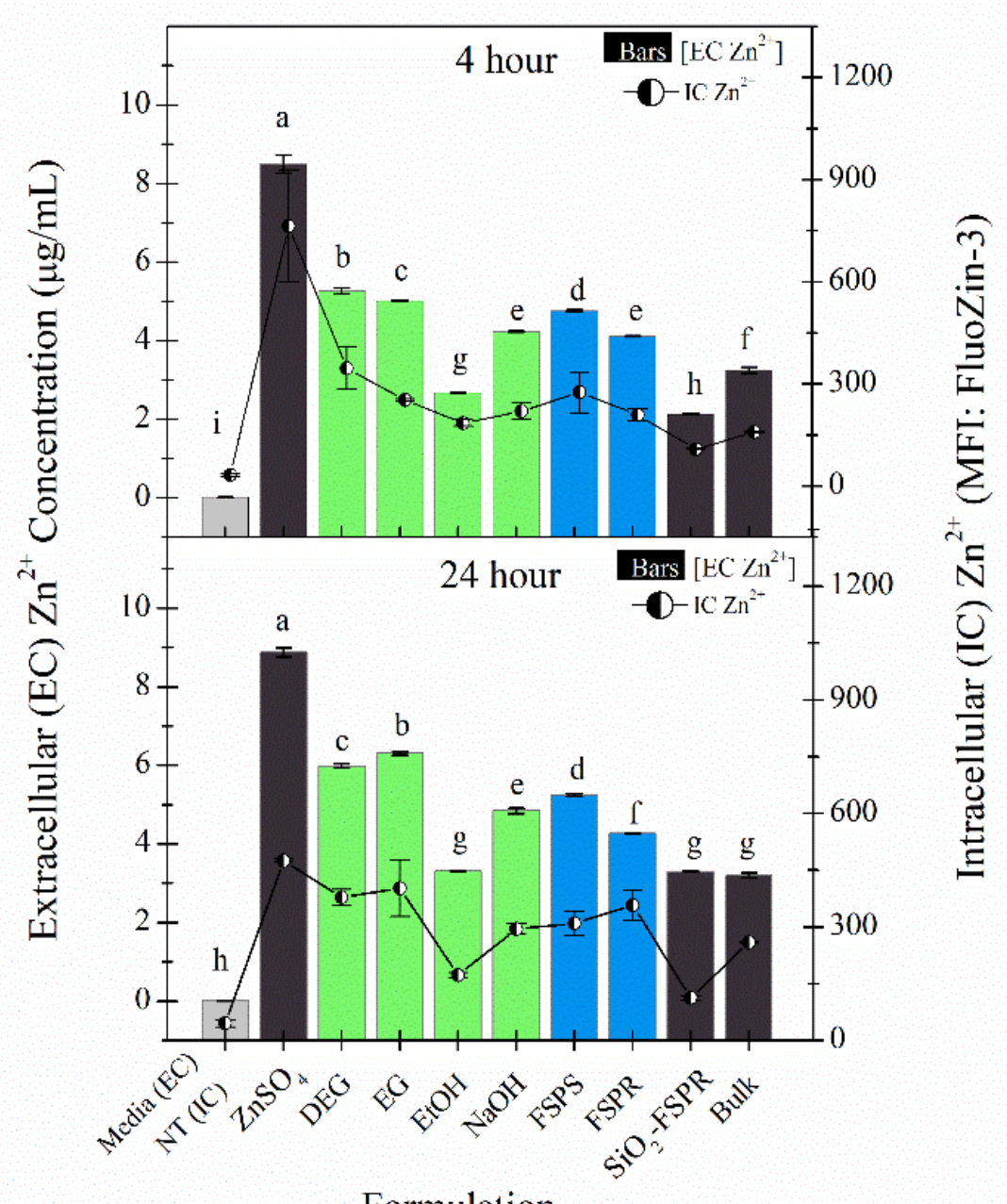

Formulation

Figure 3.4 nZnO formulations display similar dissolution kinetic trends in cellular media at 4 and 24 hours. Extracellular (EC) $\mathrm{Zn}^{2+}$ concentrations $(\mu \mathrm{g} / \mathrm{mL})$ measured via ICP-MS (bars and left $y$-axis) and intracellular $\mathrm{Zn}^{2+}$ concentrations measured via flow cytometry and expressed as mean fluorescence intensity (MFI) of the zinc specific dye FluoZin-3 AM (line graphs and right $y$-axis) evaluated at 4 hours (top graph) and 24 hours (bottom graph). Control samples, designated as the left most bar or line graph symbol were RPMI-based cellular media (EC assay) and NT cells (Intracellular (IC) assay). Statistical analysis was performed for the extracellular zinc measurements (histogram bars) using repeated measures analysis of variance and model-based means post hoc test $(p<0.05)$ with differing letters denoting statistical significance. Histogram bars or line graph circles represent the average of $n=4$ replicates with error bars depicting s.e. 


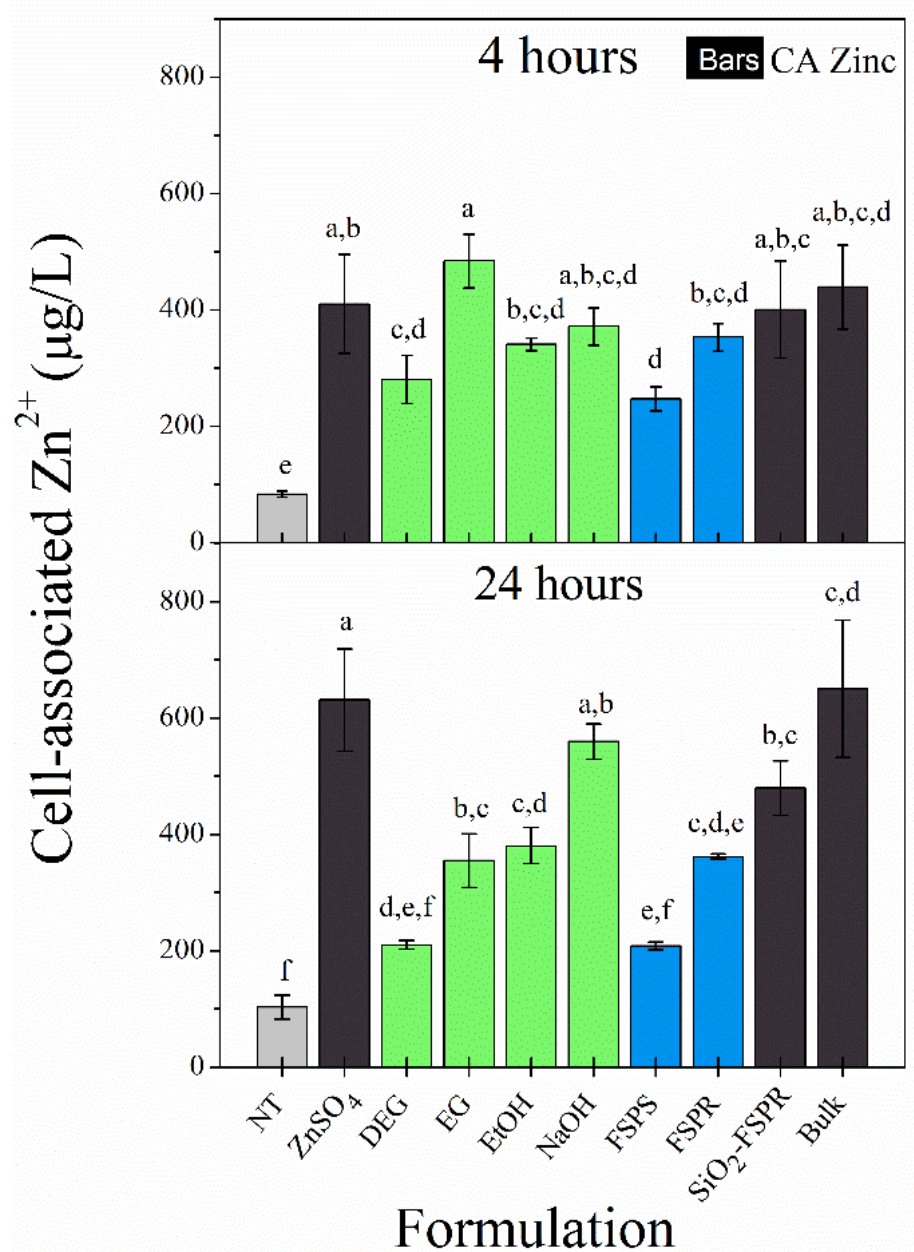

Figure 3.5 Cell-associated (CA) $\mathrm{Zn}^{2+}$ concentrations ( $\mu \mathrm{g} / \mathrm{L}$ ) measured at 4 and 24 hours via ICP-MS. A control sample designated as the left most grey bar, were nontreated cells grown in cellular media. Statistical analysis was performed using repeated measures analysis of variance and model-based means post hoc test $(p<0.05)$ with differing letters denoting statistical significance. Histogram bars represent the average of $n=4$ replicates with s.e. error bars. 

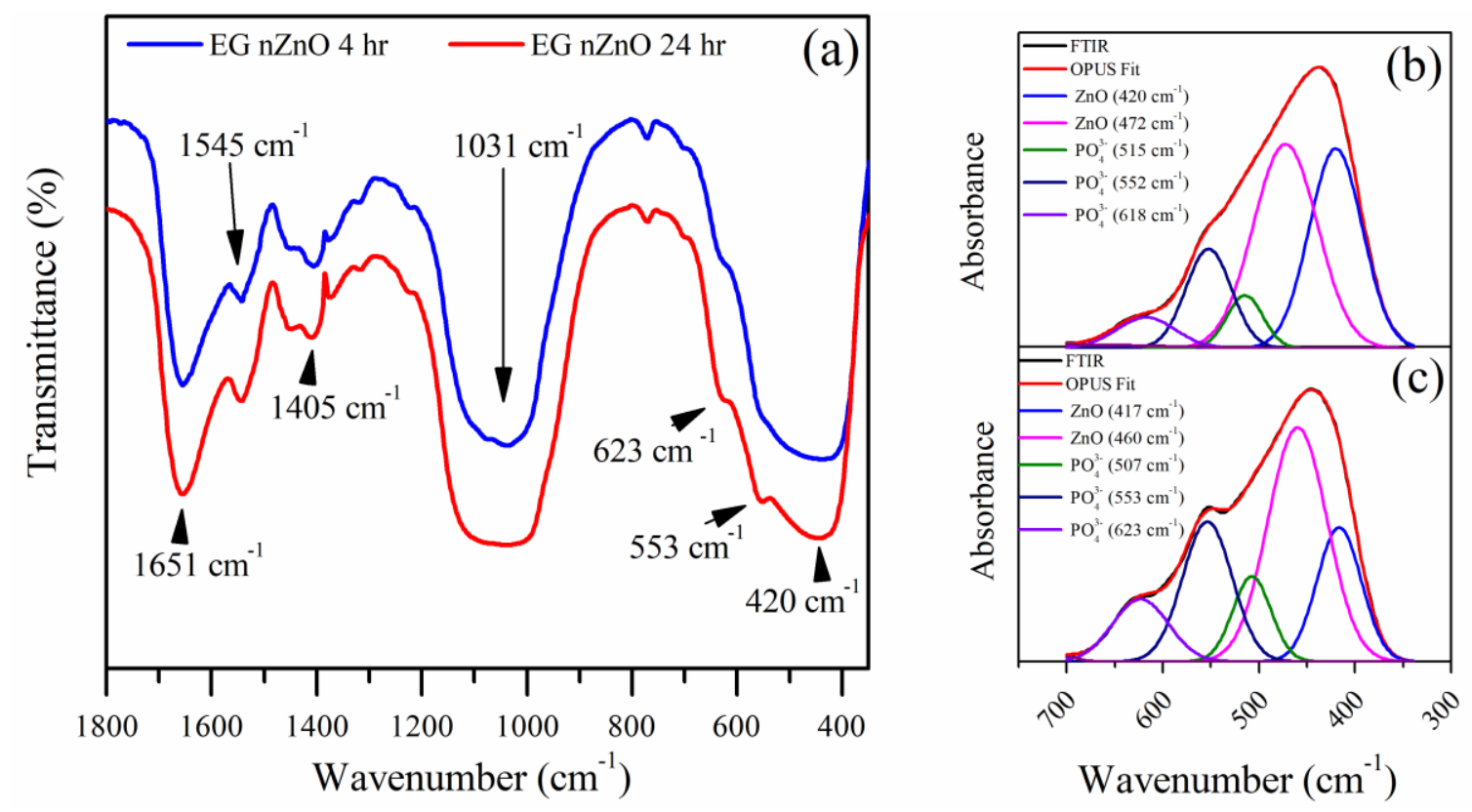

Figure 3.6 Representative samples highlighting the FTIR region from 1800 to 350 $\mathrm{cm}^{-1}$ and the $\mathrm{ZnO}$ peak deconvolution. The figure includes (a) FTIR spectra from 1800 to $350 \mathrm{~cm}^{-1}$ for the EG nZnO sample at 4 hours (blue) and 24 hours (red) and peak deconvolution of the broad FTIR band from $750-350 \mathrm{~cm}^{-1}$ for the $\mathrm{EG} \mathrm{nZnO}$ sample at (b) 4-hour and (c) 24-hour time points. Samples were introduced to cellular media at a concentration of $32 \mu \mathrm{g} / \mathrm{mL}$ and incubated for the indicated time points. After incubation, the dispersions were centrifuged and the precipitate retained and dried overnight at $60^{\circ} \mathrm{C}$. 

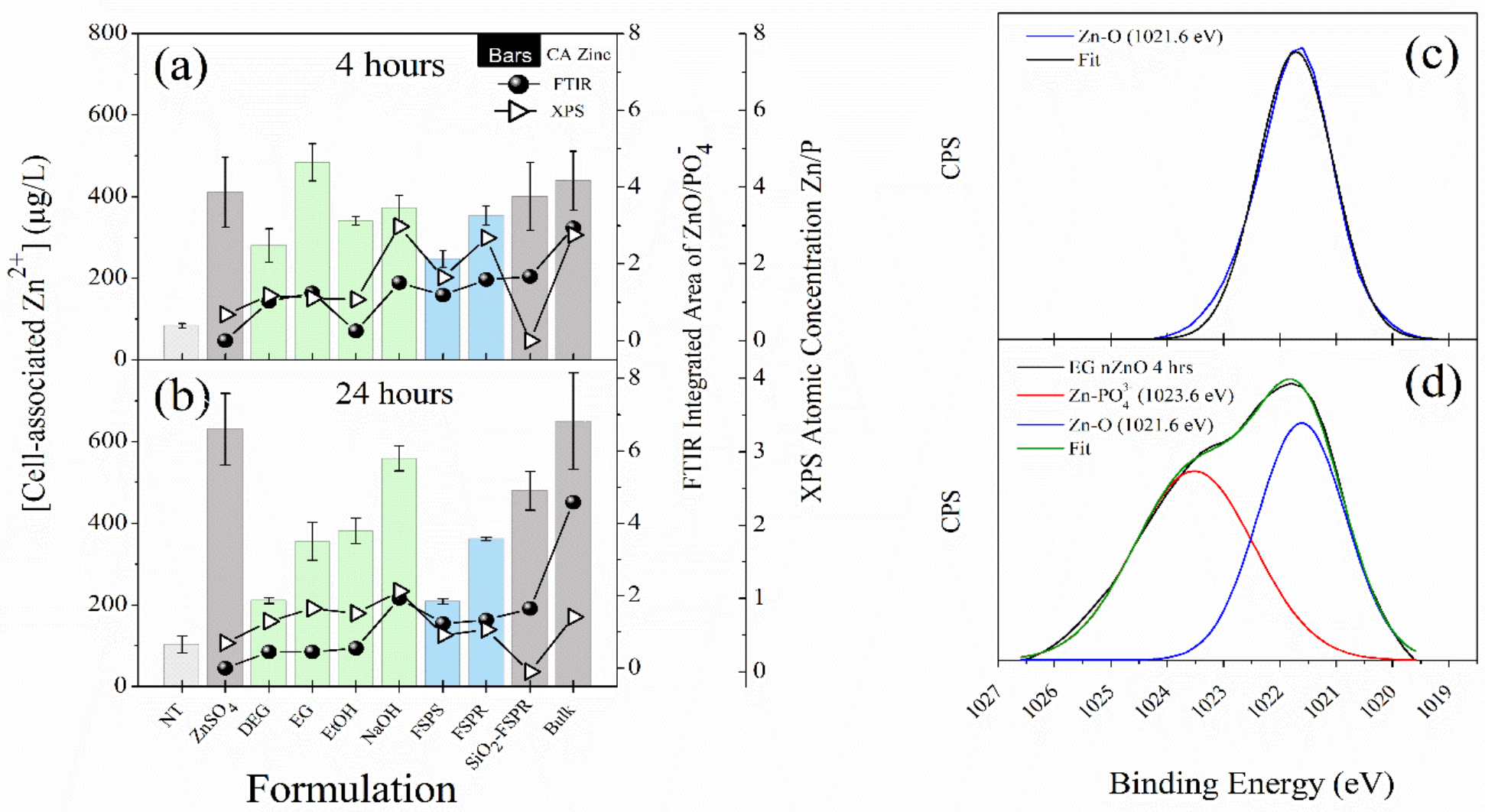

Figure 3.7 The integrated area ratio of $\mathrm{ZnO}$ to PO43- from the FTIR spectra and the atomic concentration ratio of $\mathrm{Zn} / \mathrm{P}$ from the XPS survey spectra of the insoluble zinc amorphous precipitates isolated from nZnO dispersions in cellular media post incubated for 4 and 24 hours. The left-hand side of the figure represents the integrated area ratio of $\mathrm{ZnO}$ to $\mathrm{PO}_{4}{ }^{3-}$ from the FTIR spectra (line graphs with circles and first right $\mathrm{y}$-axis) and the atomic concentration ratio of $\mathrm{Zn} / \mathrm{P}$ from the XPS survey spectra (line graphs with tringles and second right y-axis) evaluated at (a) 4 hours and (b) 24 hours. The faded histogram bars represented the $\mathrm{CA}$ zinc results presented in Fig. 3.5 and are included for reference. The right-hand side of the figure represents the XPS spectra from $1027 \mathrm{eV}$ to $1018.5 \mathrm{eV}$ illustrating the deconvolution of the $\mathrm{Zn} 2 \mathrm{p} 3 / 2$ peak for the (c) EG nZnO as prepared sample and the (d) EG nZnO in cellular media at the 4-hour time point. 

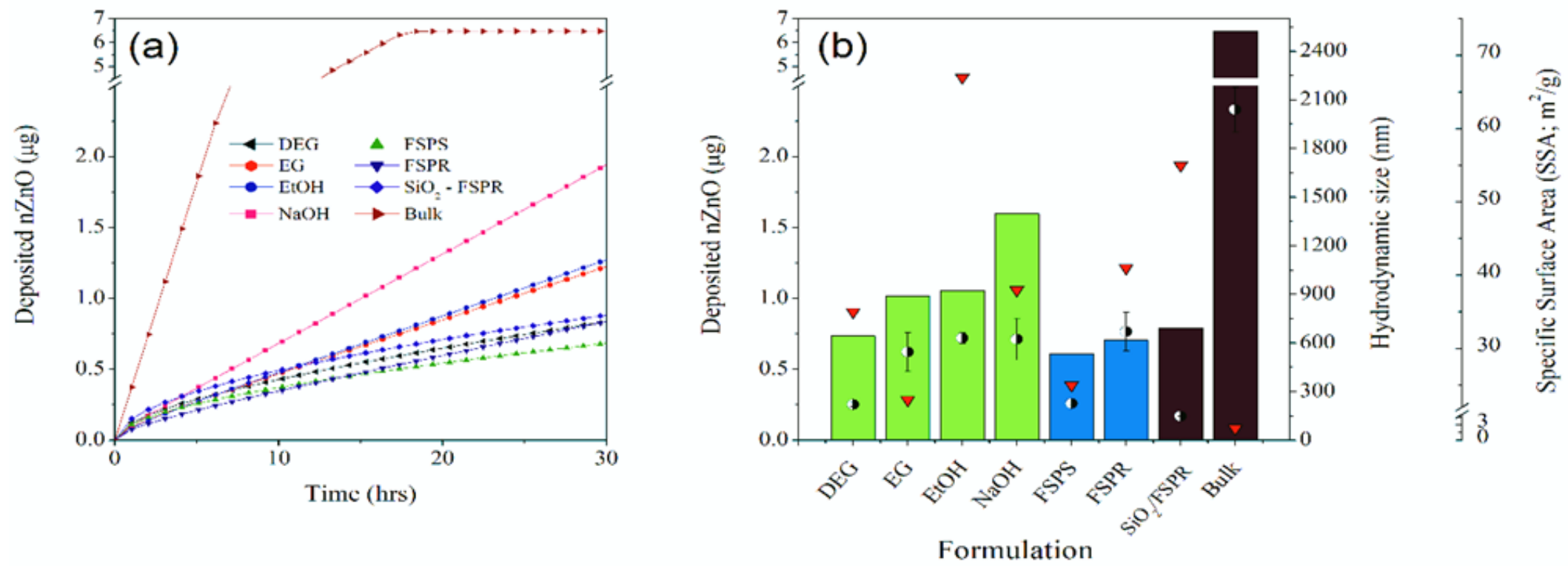

Figure 3.8 The time-dependent deposition $\mathrm{nZnO}$ onto the bottom of a well in a 96-well culture plate as determined by ISDD dosimetry modelling for the individual $\mathrm{nZnO}$ formulations. The total amount of $\mathrm{nZnO}$ introduced to the cellular media was 6.48 $\mu \mathrm{g}(32.4 \mu \mathrm{g} / \mathrm{mL}$ introduced into $0.2 \mathrm{~mL}$ of $\mathrm{RPMI}-b a s e d$ cellular media). The curves in (a) represent the calculated $\mathrm{nZnO}$ deposition over a 30-hour period. The histogram bars (left $y$-axis) in (b) demonstrate the modelled concentration of $\mathrm{nZnO}$ deposited at the 24-hour time point with the half white/half black circles (firs right y-axis) correlating to hydrodynamic size and the red triangles (second right y-axis) representing the specific surface area (SSA) as measured by BET. Error bars in (b) represent s.e. with $n=3$ replicates. 
(a)

\begin{tabular}{ccc}
\hline \multirow{2}{*}{ Variable } & \multicolumn{2}{c}{ Rotated Loadings } \\
\cline { 2 - 3 }$\left[\mathrm{IC} \mathrm{Zn}^{2+}\right]$ at 24 hours & 90 & $\mathrm{PC} 2$ \\
\cline { 2 - 3 }$\left[\mathrm{EC} \mathrm{Zn}^{2+}\right]$ at 24 hours & 90 & \\
ROS \% positive cells & 86 & \\
Zeta Potential & 74 & \\
{$\left[\mathrm{IC} \mathrm{Zn}^{2+}\right]$ at 4 hours } & 66 & -56 \\
Hydrosize & -55 & 84 \\
$\mathrm{Photocatalysis}_{\left[\mathrm{CA} \mathrm{Zn}^{2+}\right] \text { at } 24 \text { hours }}$ & & 54 \\
{$\left[\mathrm{CA} \mathrm{Zn}^{2+}\right]$ at 4 hours } & & 57 \\
\hline Eigenvalues & 4.50 & 2.05 \\
\% of Variation & 51 & 23 \\
\hline
\end{tabular}

(b)

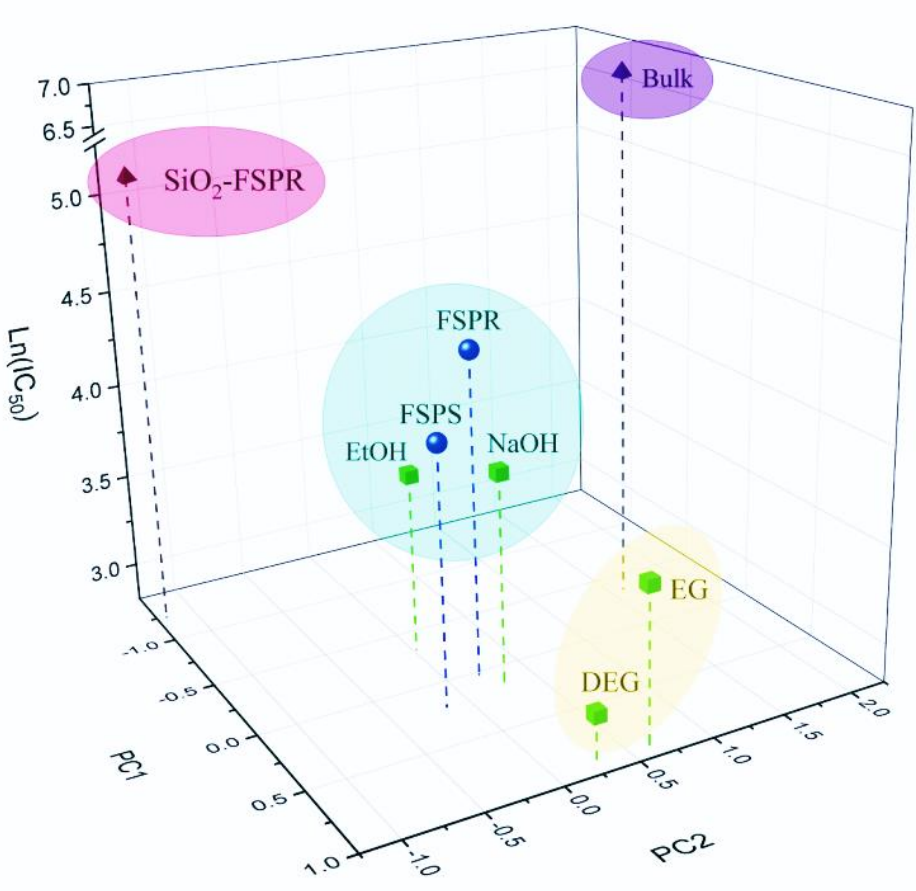

Figure 3.9 PCA model-generated values for both PC1 and PC2 and a graphical representation of the PC scores and Jurkat cells $I_{C_{50}}$ values for each evaluated sample. The table in (a) represents the loading values $(x \mathbf{1 0 0})$ for each measured variable, eigenvalues and the percent variation explained for each PC. The indicated abbreviations refer to hydrodynamic size (hydrosize), intracellular zinc [IC $\mathrm{Zn}^{2+}$ ], cell-associated-zinc [CA $\mathrm{Zn}^{2+}$ ], and extracellular zinc [EC $\mathrm{Zn}^{2+}$ ] concentrations. The green cubes (wet chemical methods), blue spheres (FSP method) and black tetrahedrons (controls samples) depicted in the 3D graph in (b) represent PC1 scores versus IC $_{50}$ values versus PC2 scores. The colored ellipses denote sample grouping based on similar PC1 and/or PC2 scores. 


\section{Electronic Supplementary Information}

\section{$\underline{\text { XRD, XPS and TEM Images }}$}

XRD (Fig. S3.1(a) and (b)), TEM (Fig. S3.1(c) through Fig. S3.1(j)) and XPS (Fig. S3.2) and were performed to verify the crystal structure, chemical composition, average size and morphology of the NP samples.

\section{Cellular Toxicity}

Table S3.1 includes $\mathrm{IC}_{50}$ values for the Jurkat leukemic cells and the primary CD4 ${ }^{+}$ T cells (see Fig. 3.1) expressed in millimolar concentration units. Also included is the calculated therapeutic index for each $\mathrm{nZnO}$ formulation.

Dissolution Kinetics for Nanopure Water Stock Solutions

Prior to conducting experiments with cells, we first investigated the kinetic behavior of the panel of $\mathrm{nZnO}$ formulations in nanopure water at concentrations identical to the NP stock solutions used in biological assays. Nanopure water dispersions containing $486 \mu \mathrm{g} / \mathrm{mL}$ of each $\mathrm{ZnO}$ formulation were prepared and stirred continuously at room temperature for a total of 24 hours. At several time points, individual aliquots were extracted and prepared for analysis with ICP-MS. The results of this analysis can be seen in Fig. S3.4. All the samples exhibit similar kinetic behavior throughout most of the experimental time frame. The kinetic pattern generally suggests that the dissolution rate of the NPs is faster from 0.5 to 6 hours and then decreases gradually as the process approaches a dynamic equilibrium. Additionally, this data directly correlates with the measured hydrodynamic sizes of the NP agglomerates in water $\left(\mathrm{R}^{2}=0.88\right)$ validating experimental evidence for the dependence of measured $\mathrm{Zn}^{2+}$ concentration on hydrodynamic size. While these results cannot be used to make inferences as to dissolution kinetics of the $\mathrm{nZnO}$ under 
biological assay conditions, these results confirm that at early time points, dissolution of all $\mathrm{nZnO}$ samples demonstrate similar kinetic behavior.

\section{$\underline{\text { FTIR and FTIR Peak Deconvolution }}$}

For this assay, the evaluated samples were incubated in RPMI-based cellular media at a concentration of $32.4 \mu \mathrm{g} / \mathrm{mL}$ and sampled at 4 and 24 hours. Post incubation, the resulting precipitate was isolated and dried overnight. Figure S3.5 illustrates the vibrational modes present for all samples at 4- and 24-hours. The graphs in Fig. S3.6 represent the deconvolution of the broad $\mathrm{ZnO}$ band present at approximately $350-700 \mathrm{~cm}^{-1}$ for all samples at the 24-hour time point. To eliminate zinc phosphate peak overlap with zinc oxide normal modes (Table S3.3), only those peaks identified below $500 \mathrm{~cm}^{-1}$ were included in the ratio of crystalline $\mathrm{ZnO}$ to phosphate $\left(\mathrm{ZnO} / \mathrm{PO}_{4}{ }^{3-}\right)$ calculations. Likewise, only the integrated area of the broad phosphate band at $1031 \mathrm{~cm}^{-1}$ was considered in the ratio to eliminate any interference from $\mathrm{ZnO}$ in the phosphate determinations.

\section{$\underline{\text { XPS Spectra of Samples in Cellular Media }}$}

XPS was used to confirm the chemical compostion for all samples post incubation in cellular media for 4 and 24 hours (Fig. S3.7). Survey spectra illustrated the presence of zinc, oxygen, nitrogen, carbon and phosphorous in all samples. The $\mathrm{SiO}_{2}-\mathrm{FSPR}$ sample also contains silica as expected.

$\underline{\text { TEM }}$

The following figures, (Fig. S3.8 through Fig. S3.15), represent low and high resolution TEM images obtained for the samples described above in the FTIR section. Low resolution images revealed that large $\mathrm{nZnO}$ agglomerates (darker areas) were surrounded by an organic matrix. The higher resolution scans provided confirmation that the darker 
regions of the TEM images were composed of primarily crystalline $\mathrm{ZnO}$ due to the presence of observed lattice fringes representing the individual planes of the ordered $\mathrm{ZnO}$. $\underline{\text { Principal Component Analysis }}$

Table S3.2 describes the results of the linear contrast model predictions for Jurkat cells $\mathrm{IC}_{50}$ as a function of PC1 and PC2. 
Tables and Figures - Electronic Supplementary Material
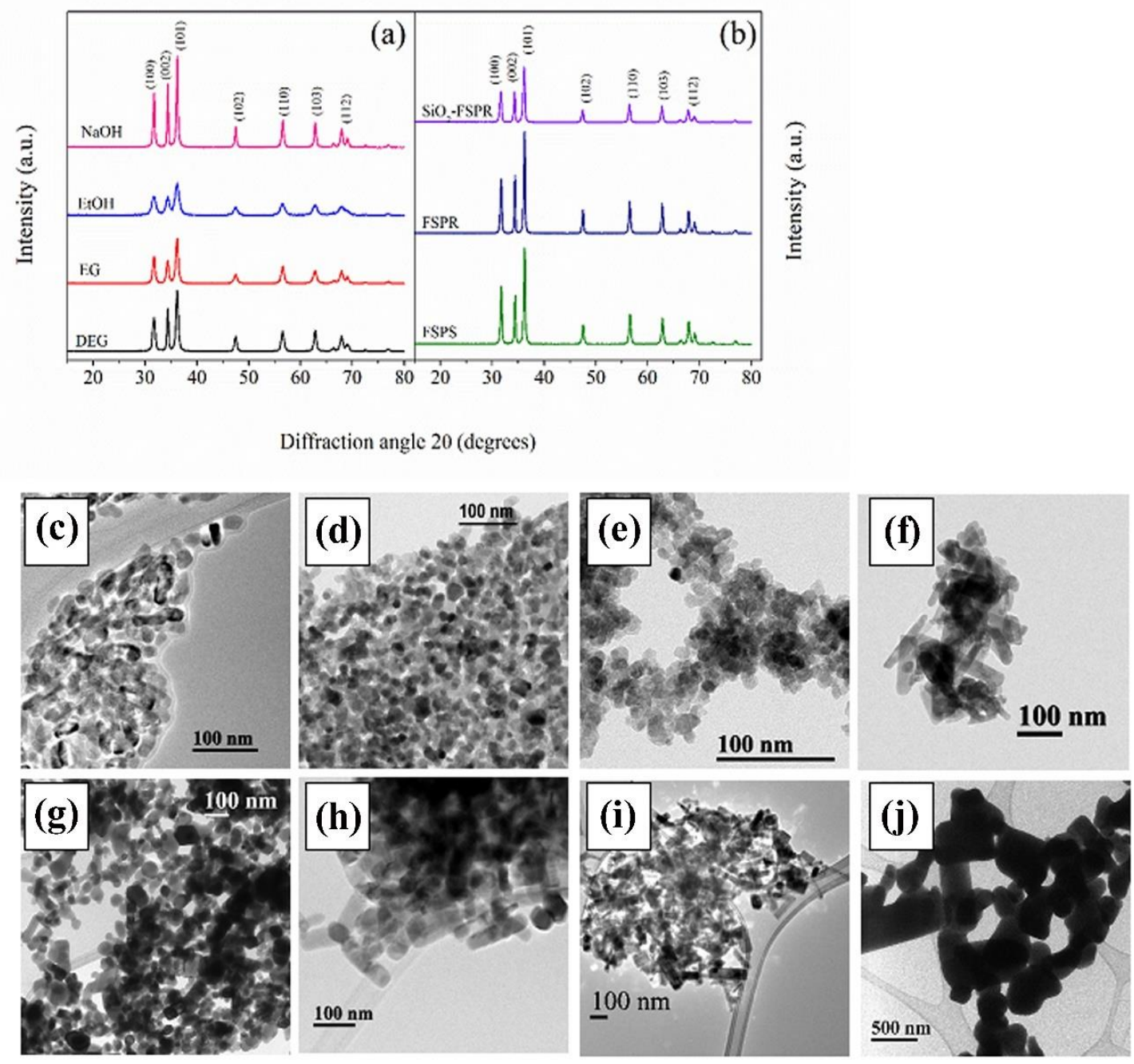

Figure S3.1 Crystal phase composition, size and morphology characterization for nZnO NPs. XRD spectra for (a) nZnO synthesized through wet chemical synthesis methods and (b) nZnO synthesized through flame spray pyrolysis (FSP) synthesis methods. TEM images for (c) DEG, (d) EG (e) EtOH, (f) NaOH, (g) FSPS, (h) FSPR, (i) $\mathrm{SiO}_{2}$-FSPR and (j) bulk samples were used to identify morphology and average NP size and distribution. 
Table S3.1 The Jurkat and primary $\mathrm{CD4}^{+} \mathrm{T}$ cell $\mathrm{IC}_{50}$ values converted to millimolar (mM) concentrations and the calculated therapeutic index.

\begin{tabular}{|c|c|c|c|}
\hline \hline Formulation & $\begin{array}{c}\text { Jurkat Cell } \\
\text { IC50 }(\mathrm{mM})\end{array}$ & $\begin{array}{c}\text { Primary T Cell } \\
\text { IC50 }(\mathrm{mM})\end{array}$ & $\begin{array}{c}\text { Therapeutic Index } \\
\left(\frac{T \text { cell } \text { C }_{50}}{\text { Jurkat cell IC }}\right)\end{array}$ \\
\hline DEG & 0.26 & 4.0 & 16 \\
\hline EG & 0.45 & 5.8 & 13 \\
\hline EtOH & 0.52 & 6.3 & 12 \\
\hline NaOH & 0.60 & 18.4 & 30 \\
\hline FSPS & 0.77 & 22.7 & 30 \\
\hline FSPR & 1.07 & 30.9 & 29 \\
\hline SiO2-FSPR & 2.10 & 30.9 & 15 \\
\hline Bulk & 13.07 & 30.9 & 2 \\
\hline \hline
\end{tabular}




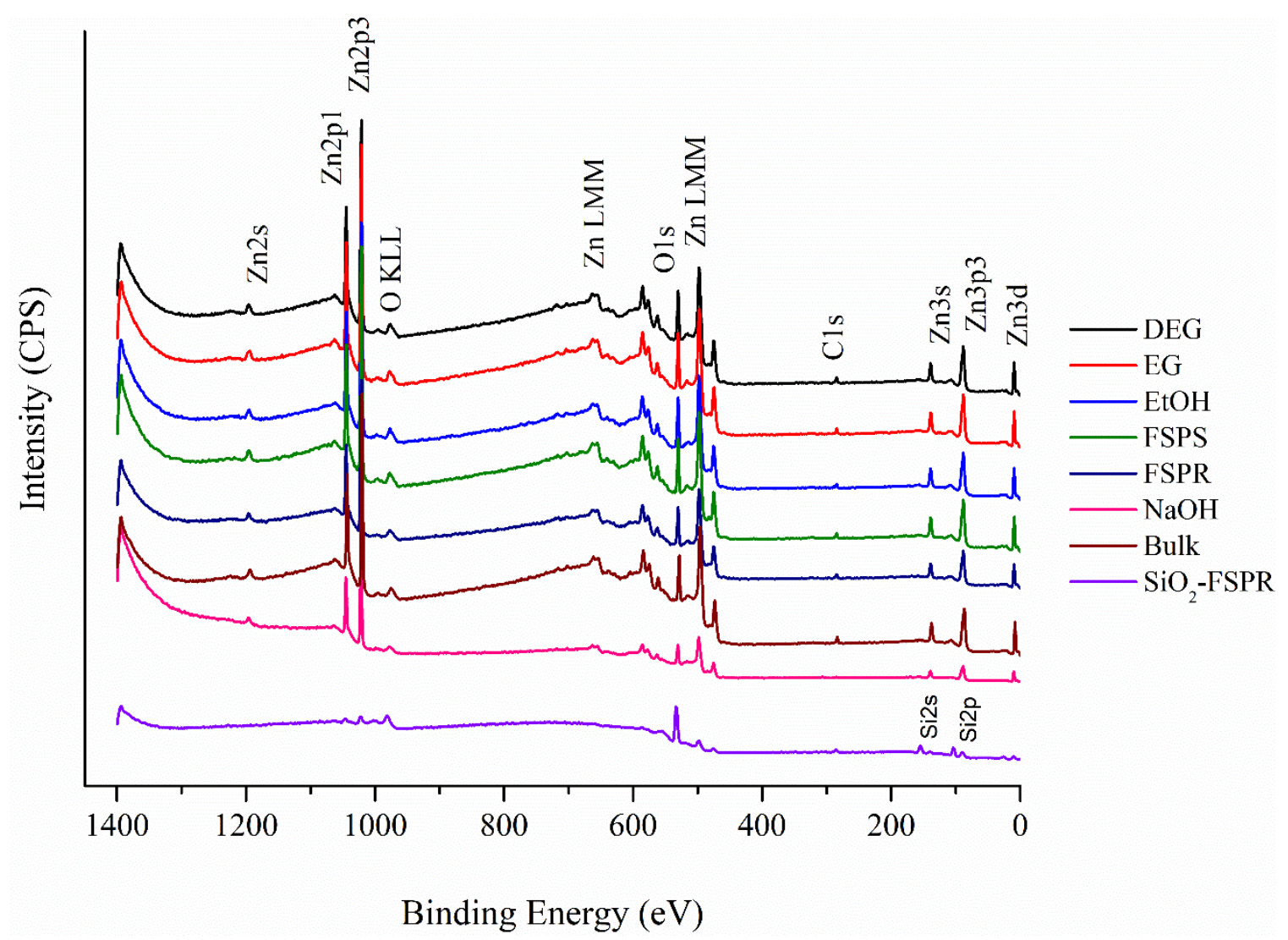

Figure S3.2 XPS survey scans for all tested powder samples 


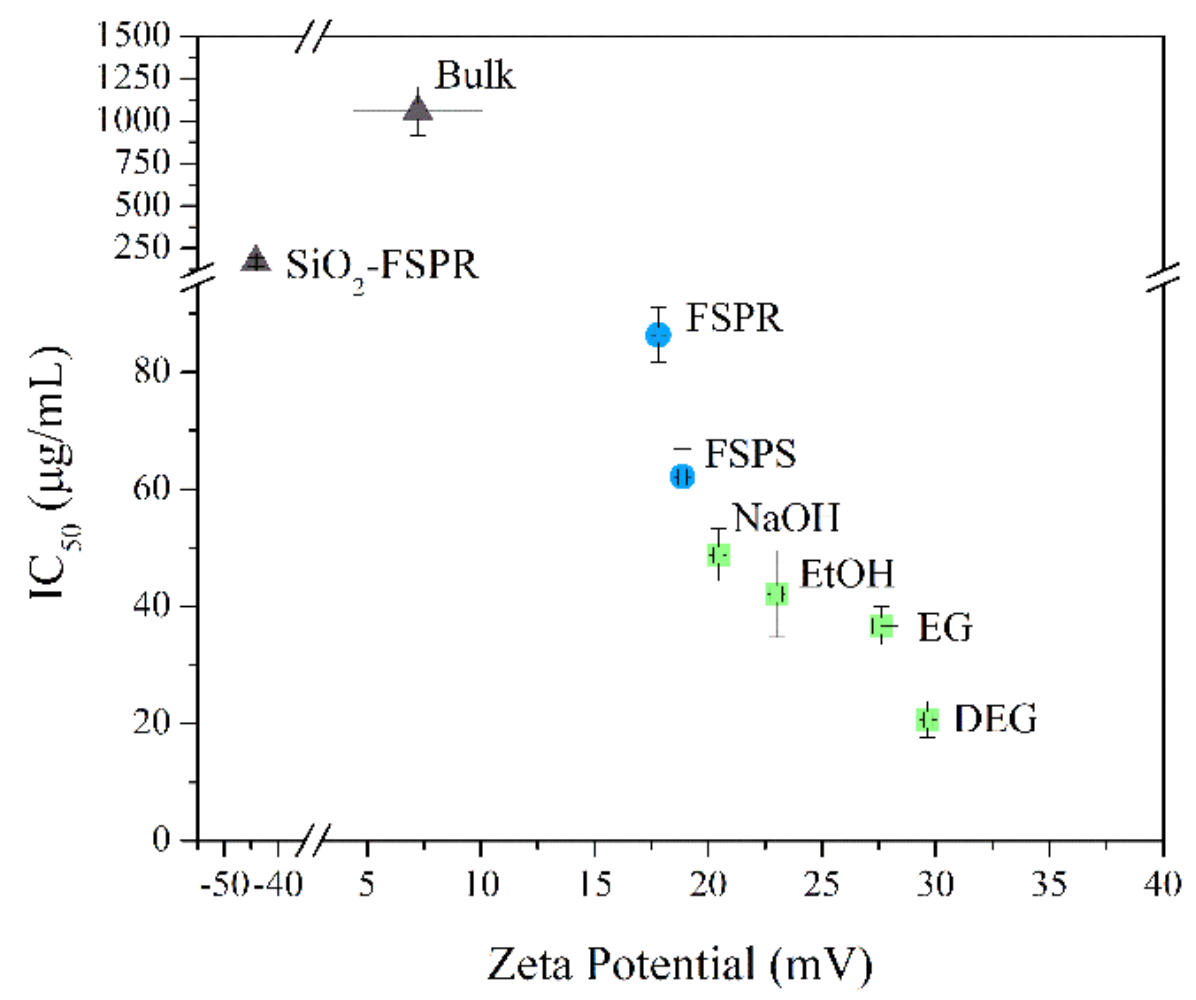

Figure S3.3 IC $\mathrm{IC}_{50}(\mu \mathrm{g} / \mathrm{mL})$ values plotted as a function of the $\mathrm{nZnO}$ zeta potential $(\mathrm{mV})$ in aqueous conditions depicting the linear correlation between the two measures variables for wet chemical and FSP synthesized nZnO. 


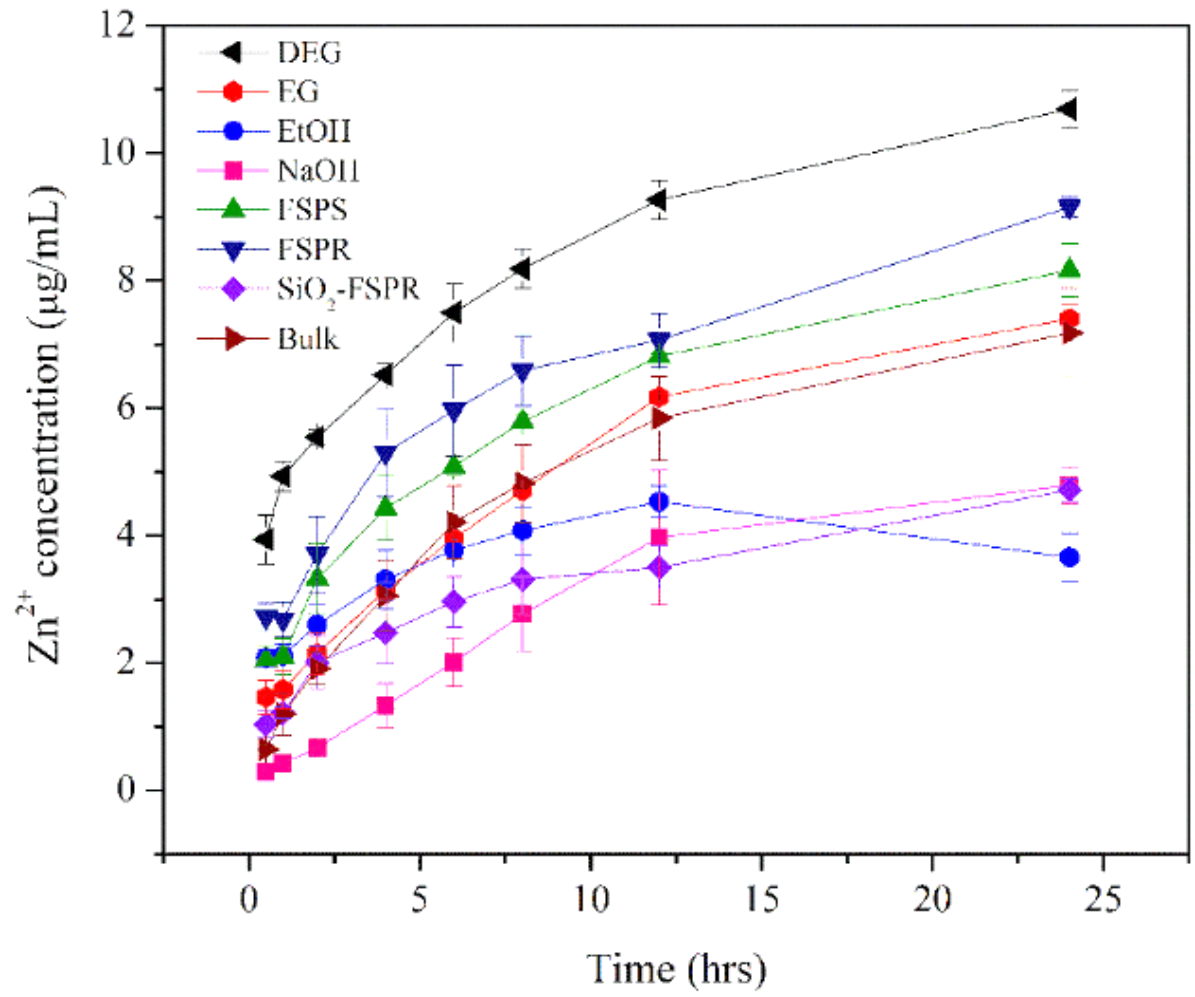

Figure S3.4 Dissolution kinetics for nanopure water dispersions for all tested formulations 


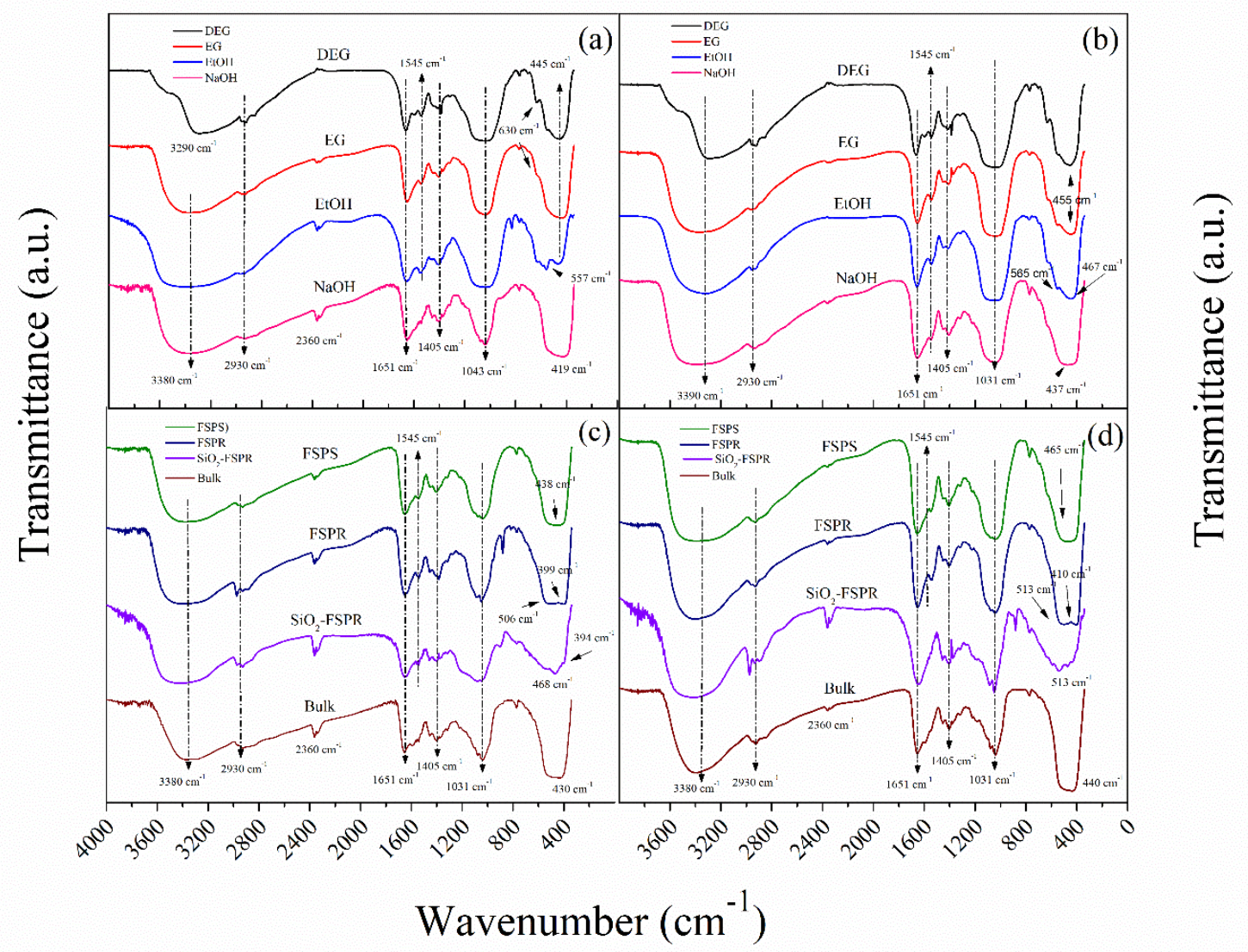

Figure S3.5 FTIR spectra obtained for the amorphous precipitate material retained at the 4-hour [(a) and (c)] and 24-hour [(b) and (d)] time points. Samples were introduced to cellular media at a concentration of $32 \mu \mathrm{g} / \mathrm{mL}$ and incubated for the indicated time points. After incubation, the dispersions were centrifuged and the precipitate retained and dried overnight at $60{ }^{\circ} \mathrm{C}$. 

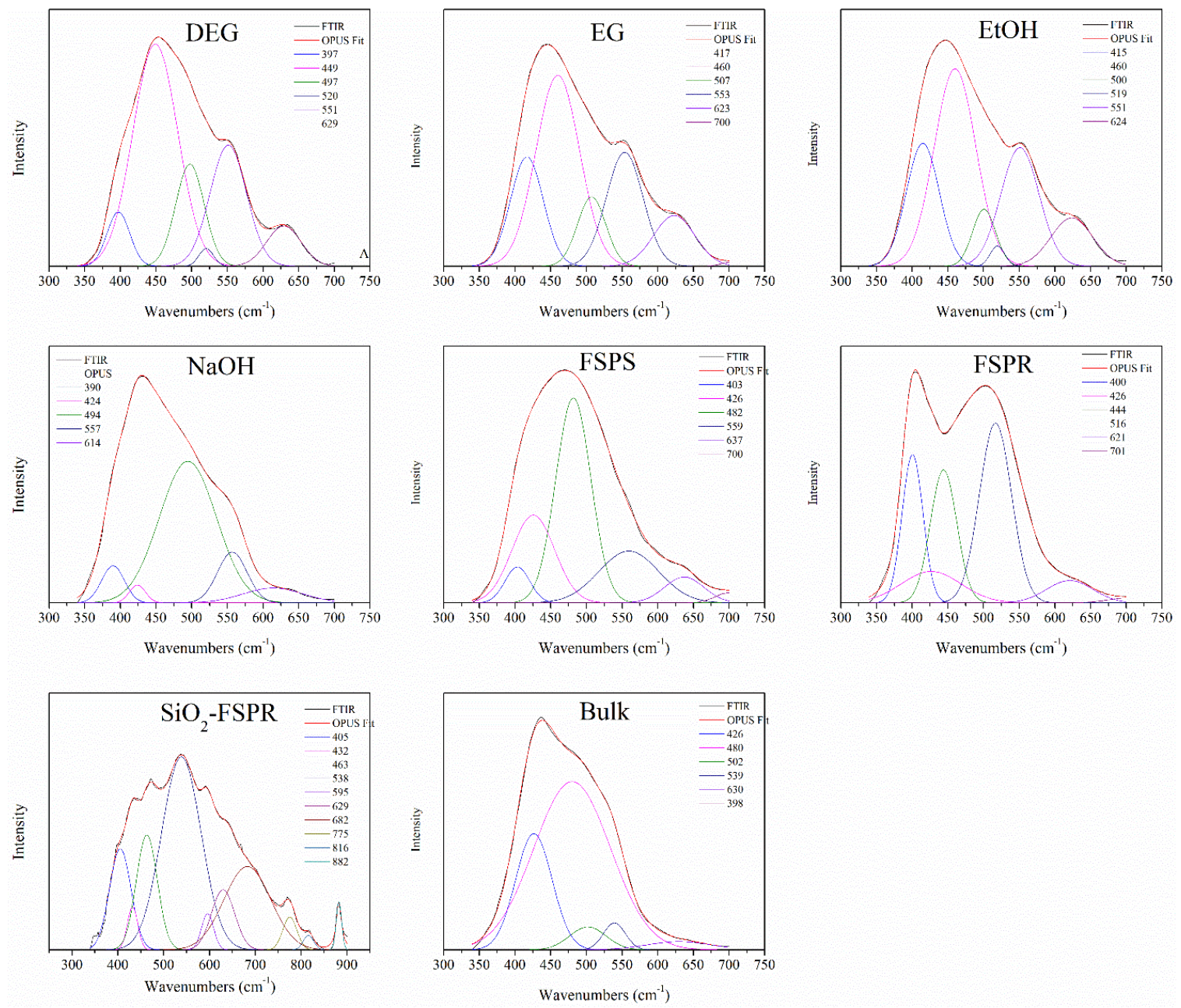

Figure S3.6 FTIR peak deconvolution results for all powdered samples post incubation in cellular media for 24 hours. Each graph is labeled with the sample name with legend numbers corresponding to the peak position (in $\mathbf{n m}$ ) for each identified peak during the deconvolution process. 

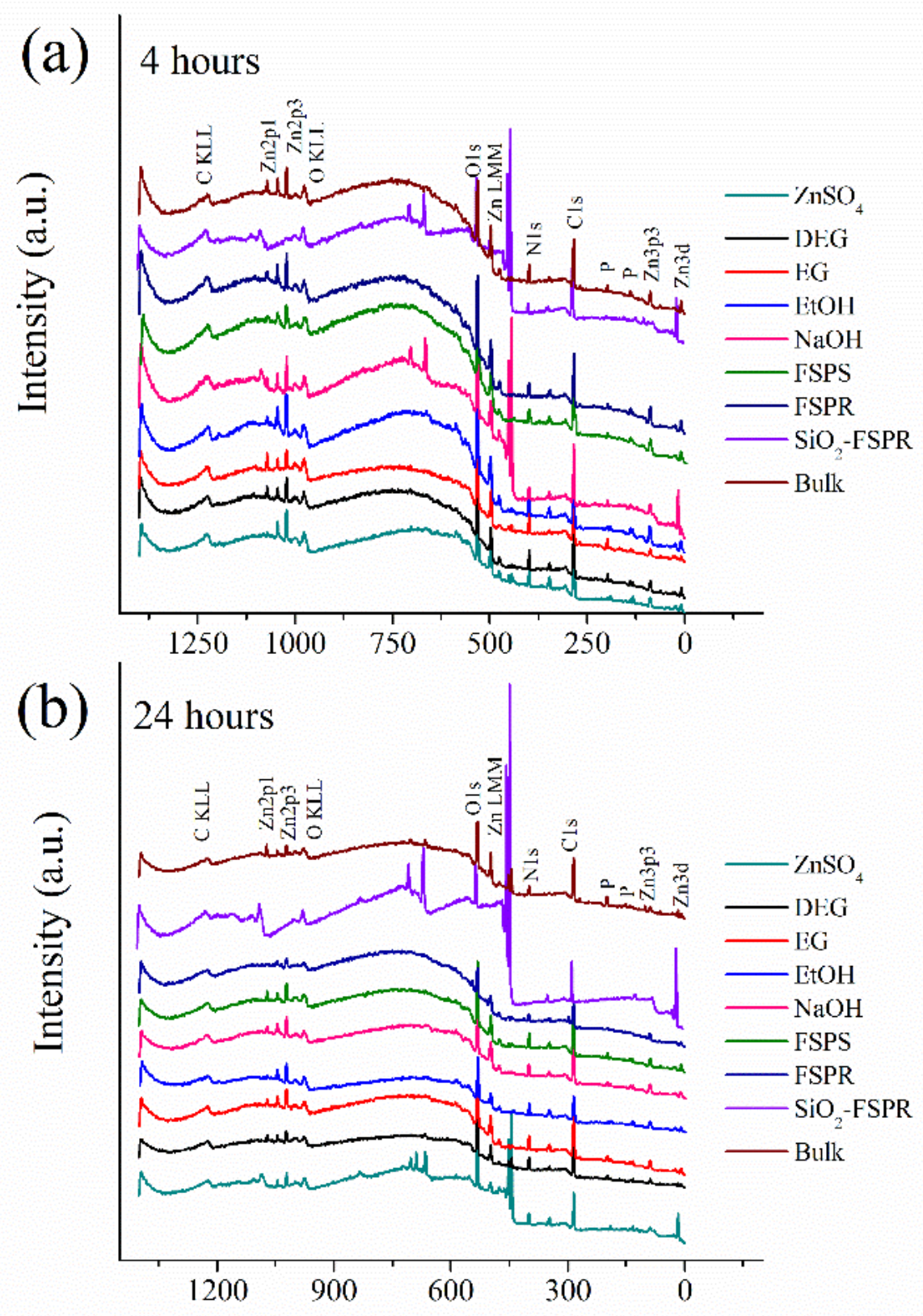

Binding energy $(\mathrm{eV})$

Figure S3.7 XPS survey scans for all tested samples incubated in cellular media for (a) 4 hours and (b) 24 hours. 

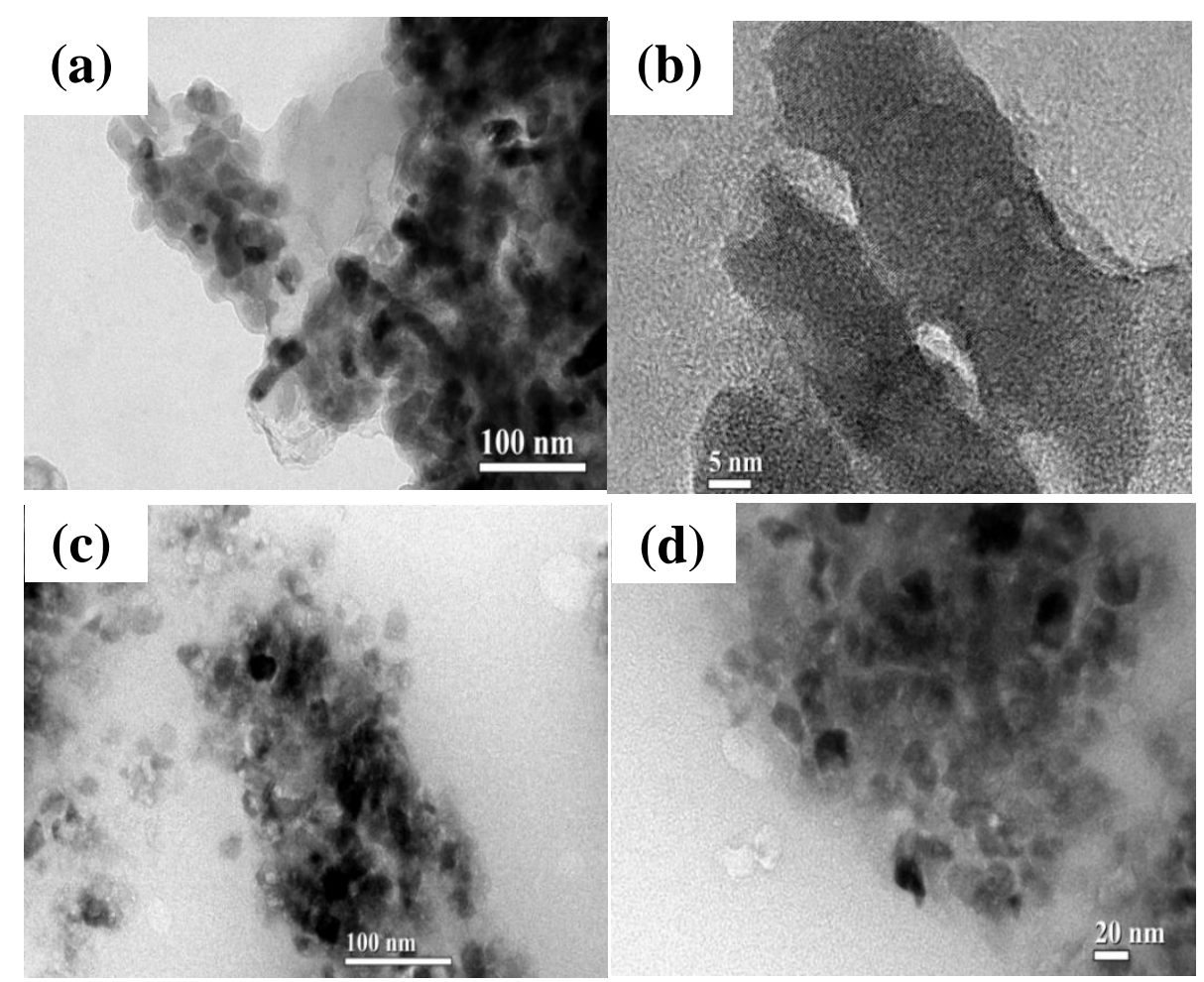

Figure S3.8 Low (a) and (c) and high (b) and (d) resolution TEM images for the DEG sample precipitates isolated from RPMI-based cellular media at (a) and (b) 4hour and (c) and (d) 24-hour time points. 


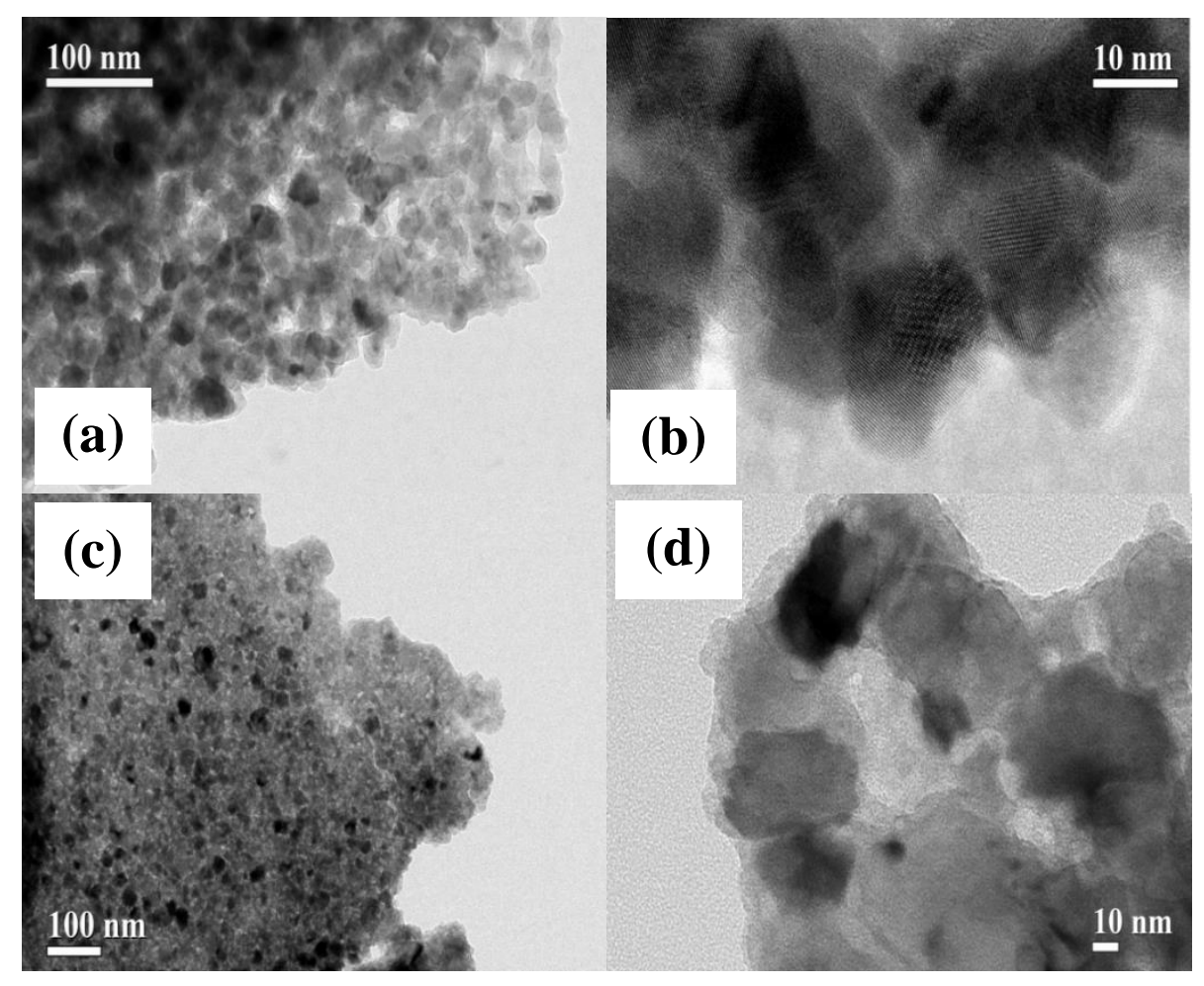

Figure S3.9 Low (a) and (c) and high (b) and (d) resolution TEM images for the EG sample precipitates isolated from RPMI-based cellular media at (a) and (b) 4-hour and (c) and (d) 24-hour time points. 

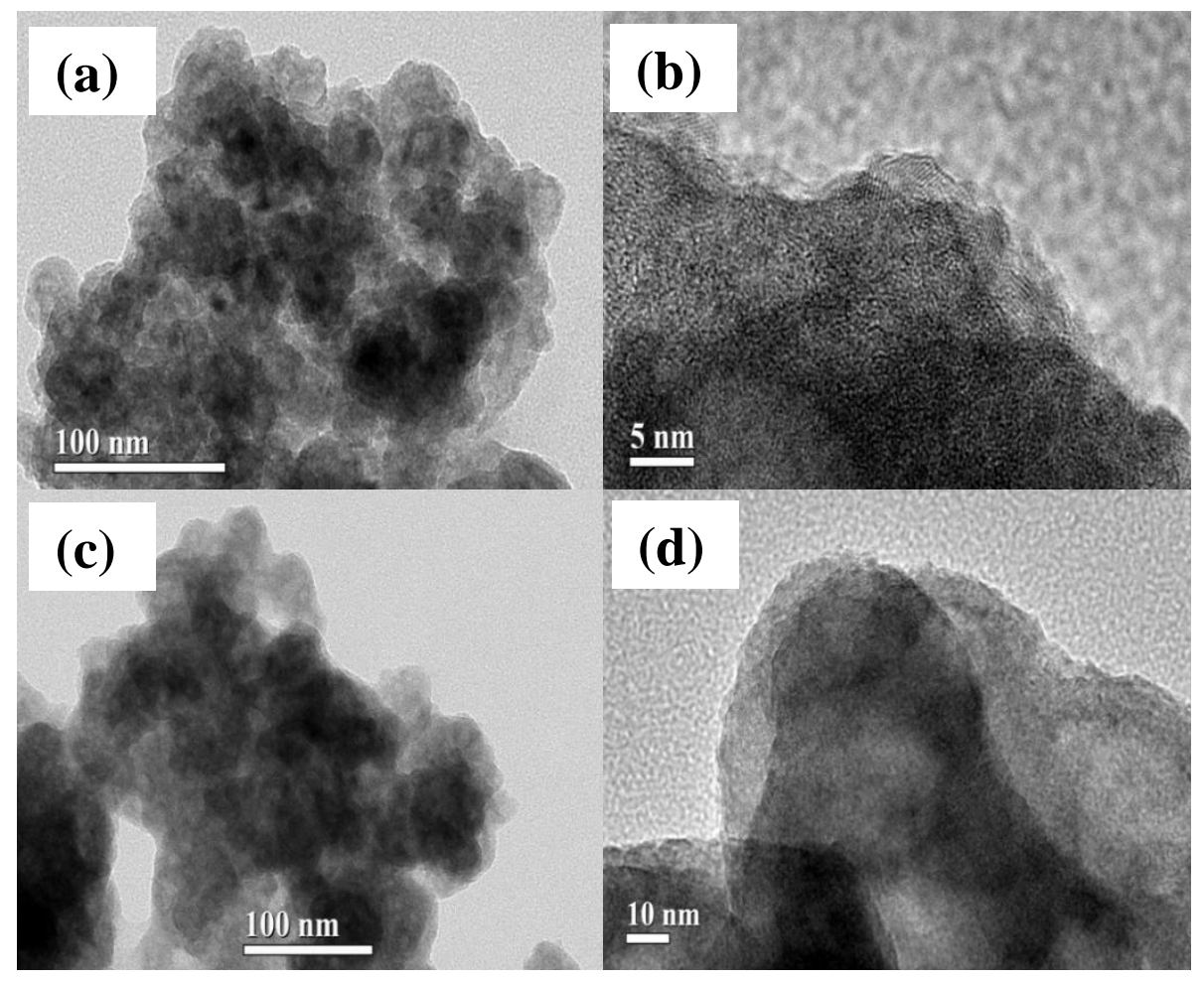

Figure S3.10 Low (a) and (c) and high (b) and (d) resolution TEM images for the EtOH sample precipitates isolated from RPMI-based cellular media at (a) and (b) 4hour and (c) and (d) 24-hour time points. 


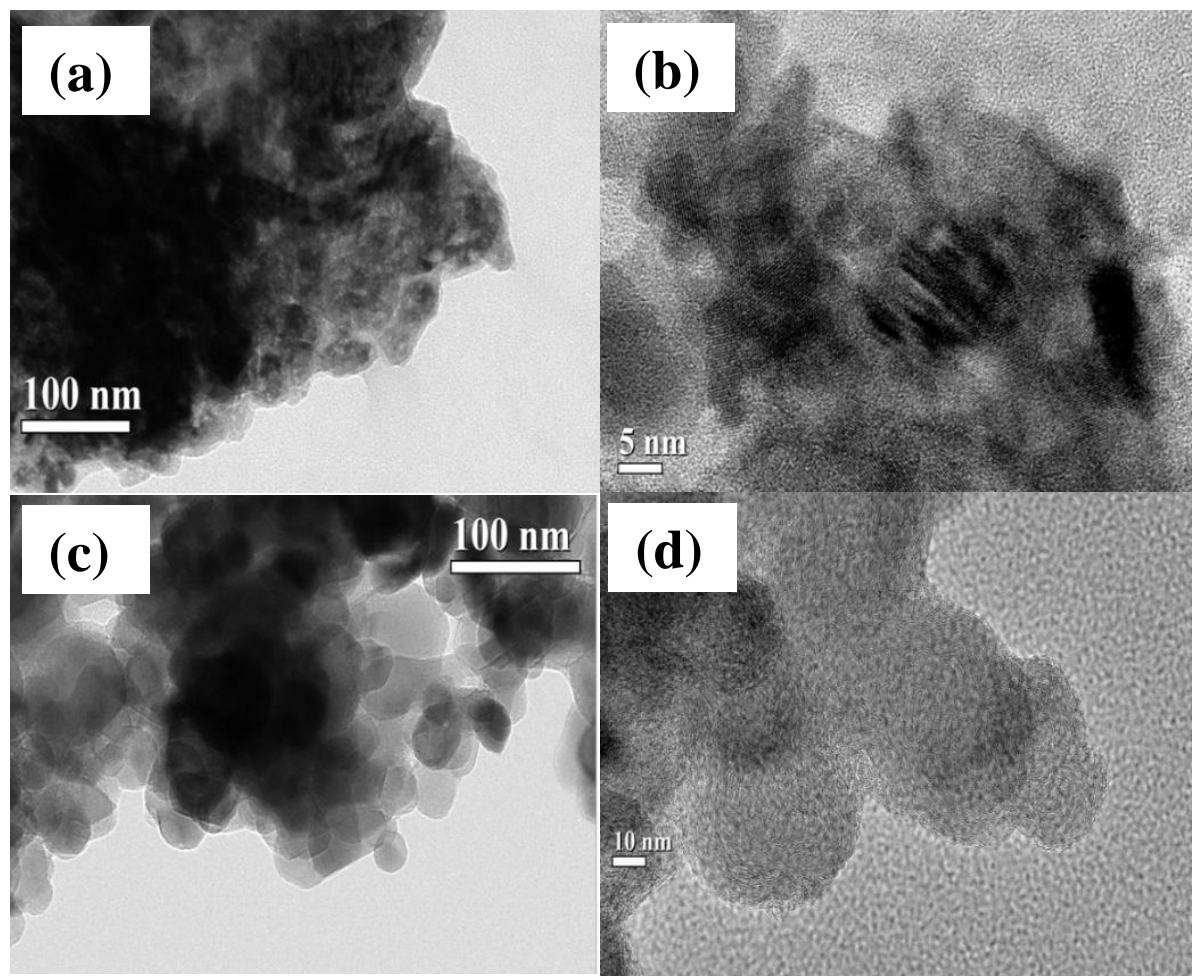

Figure S3.11 Low (a) and (c) and high (b) and (d) resolution TEM images for the NaOH sample precipitates isolated from RPMI-based cellular media at (a) and (b) 4hour and (c) and (d) 24-hour time points. 


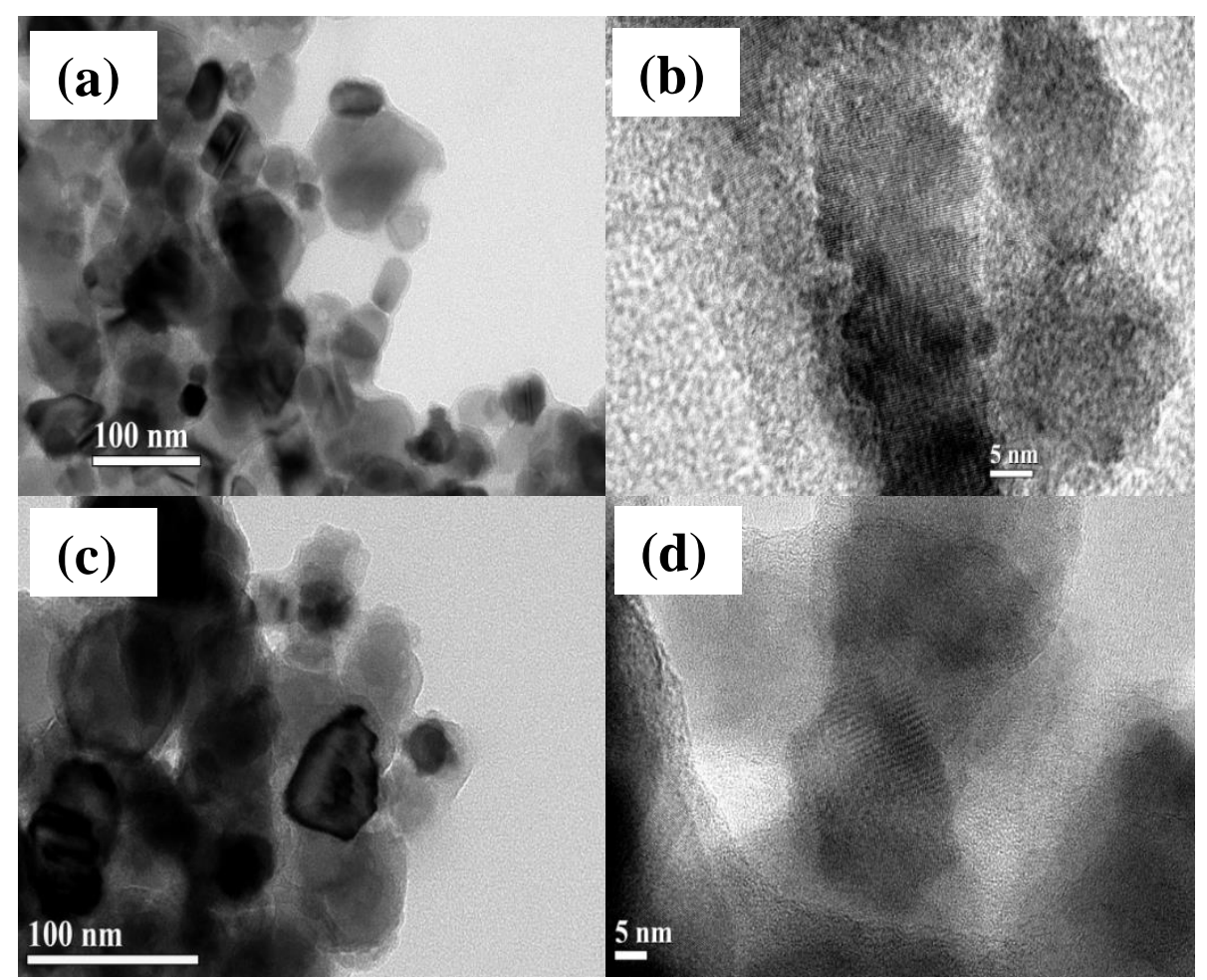

Figure S3.12 Low (a) and (c) and high (b) and (d) resolution TEM images for the FSPS sample precipitates isolated from RPMI-based cellular media at (a) and (b) 4hour and (c) and (d) 24-hour time points. 


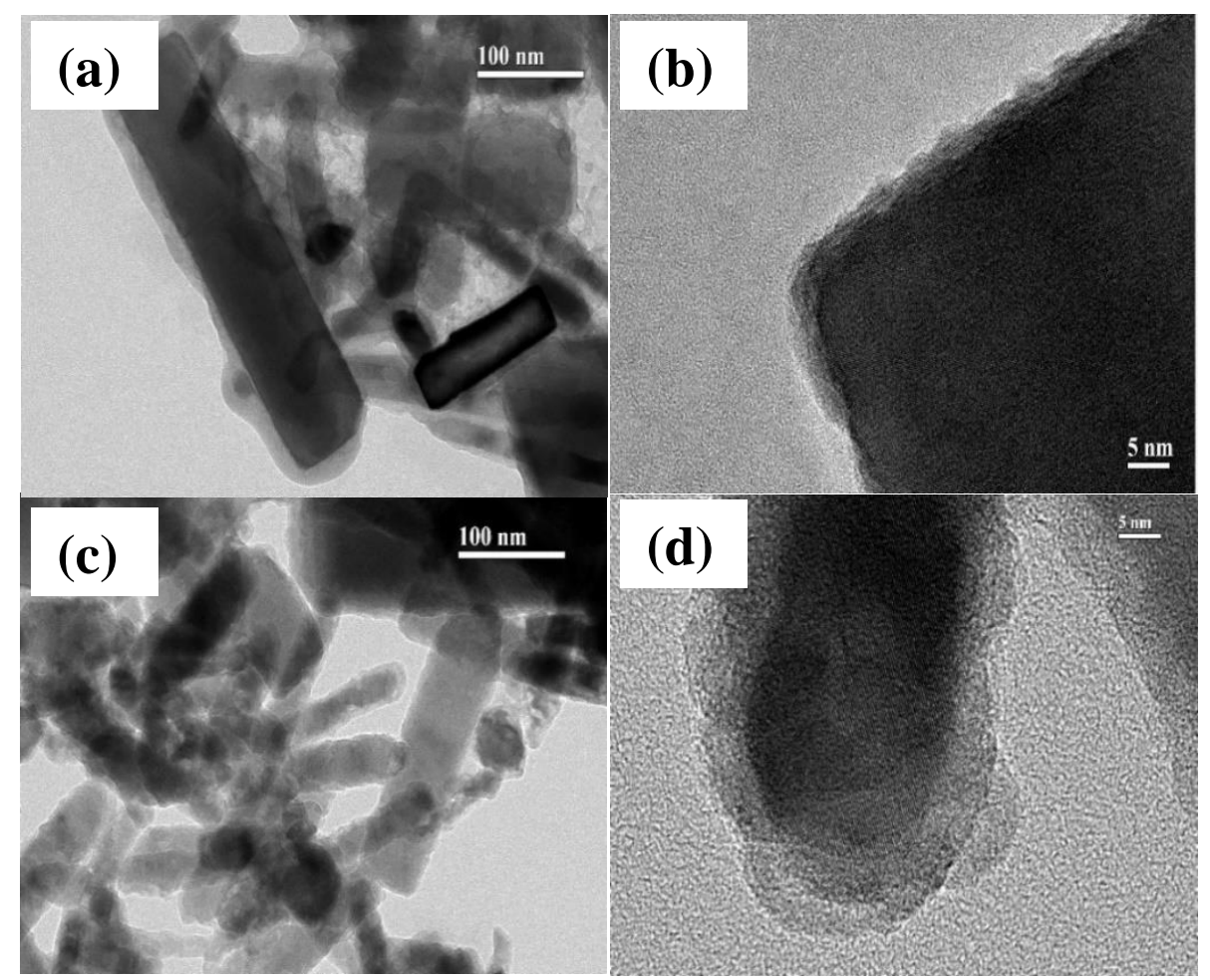

Figure S3.13 Low (a) and (c) and high (b) and (d) resolution TEM images for the FSPR sample precipitates isolated from RPMI-based cellular media at (a) and (b) 4hour and (c) and (d) 24-hour time points. 


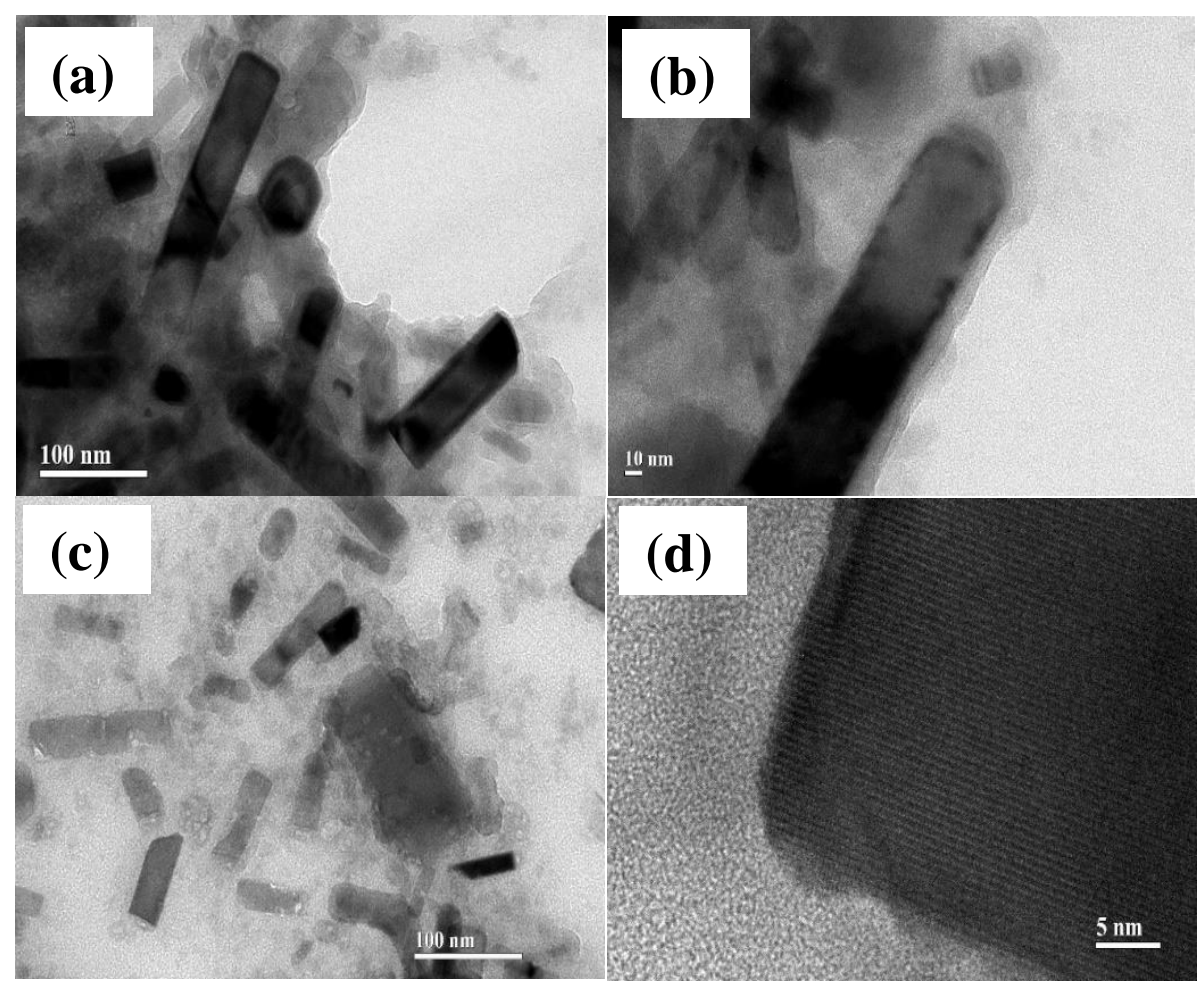

Figure S3.14 Low (a) and (c) and high (b) and (d) resolution TEM images for the $\mathrm{SiO}_{2}$ - FSPR sample precipitates isolated from RPMI-based cellular media at (a) and (b) 4-hour and (c) and (d) 24-hour time points. 


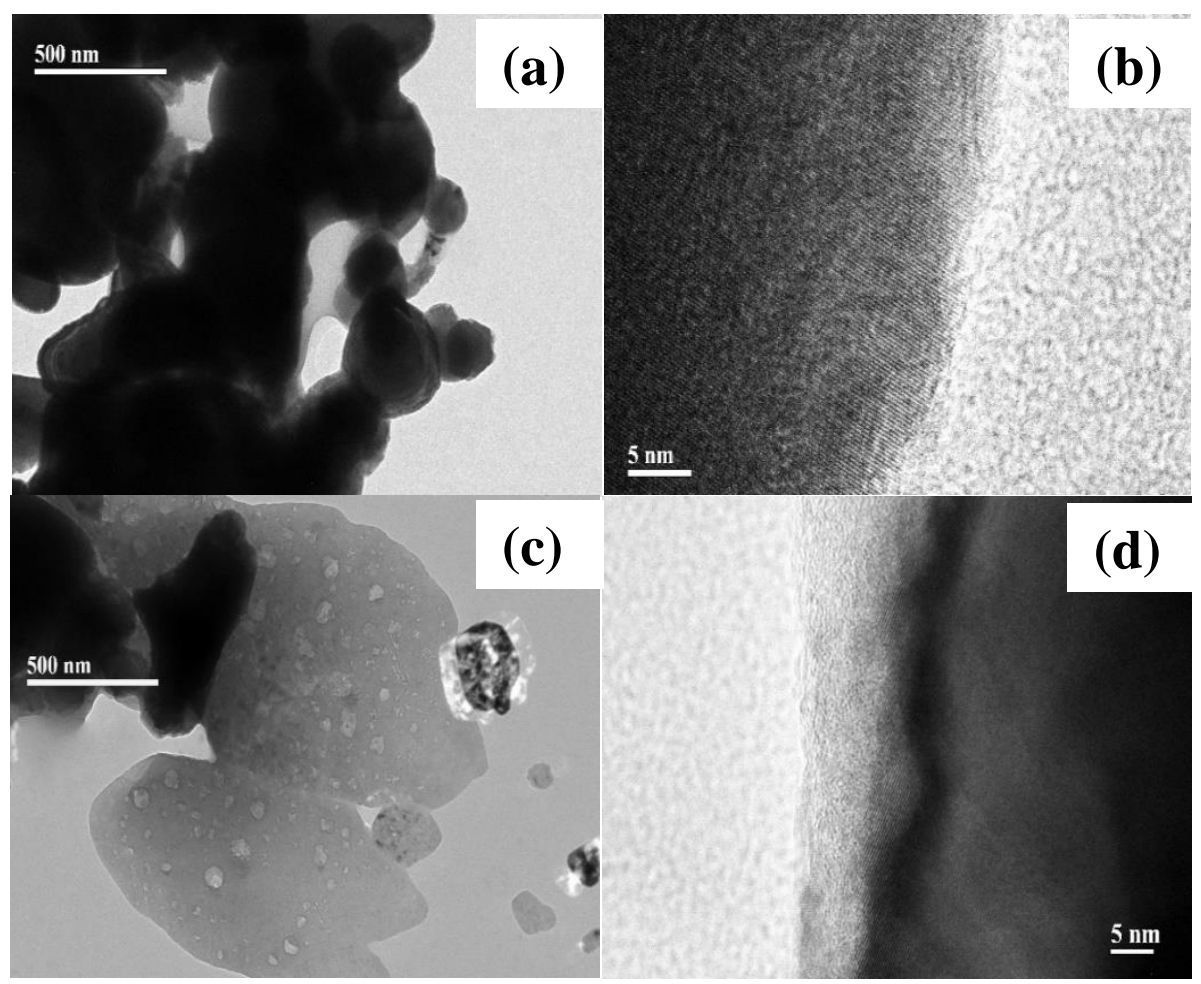

Figure S3.15 Low (a) and (c) and high (b) and (d) resolution TEM images for the Bulk sample precipitates isolated from RPMI-based cellular media at (a) and (b) 4hour and (c) and (d) 24-hour time points. 
Table S3.2 Linear regression model for $\mathrm{IC}_{50}$ as a linear function of PC1 and PC2.

\begin{tabular}{cc}
\hline Term & Estimate (S.E.) $^{\mathbf{2}}$ \\
\hline Intercept & $-0.11(0.05)$ \\
PC1 & $-0.64(0.08)$ \\
PC2 & $0.48(0.07)$
\end{tabular}

${ }^{1}$ Overall model for $\mathrm{IC}_{50}$ as a linear function of $\mathrm{PC} 1$ and $\mathrm{PC} 2$ is statistically significant $\left(F_{2,21}=39.91, p<0.0001\right)$ with 24 observations and is presented as:

$$
\text { Jurkat IC } C_{50}=-0.11-0.64(P C 1)+0.48(P C 2)
$$

${ }^{2}$ All statistically significant at $\mathbf{p}<0.05$ 
Table S3.3 Identified FTIR peaks for the 24-hour precipitate analysis after deconvolution of the broad $\mathrm{ZnO}$ band (350-750 $\mathrm{cm}^{-1}$ ) including the possible peak assignment and reference

\begin{tabular}{|c|c|c|c|c|c|c|c|c|c|}
\hline DEG & EG & $\mathrm{EtOH}$ & $\mathrm{NaOH}$ & FSPS & FSPR & $\mathrm{SiO}_{2}-\mathrm{FSPR}$ & Bulk & Peak Assignment & Reference \\
\hline \multirow[t]{2}{*}{397} & & & 390 & 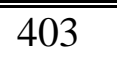 & 400 & 405 & 398 & A1(TO) & [11-3] \\
\hline & 417 & 415 & 424 & 426 & 426 & 432 & 426 & $\mathrm{E} 1(\mathrm{TO})$ & {$[1-6]$} \\
\hline \multirow[t]{2}{*}{449} & & & & & 444 & & & E2 high (Raman) & {$[2,3,6]$} \\
\hline & 460 & 460 & & 482 & & 463 & 480 & Lower surface phonon & {$[4,6]$} \\
\hline 497 & & 500 & 494 & & & & & $\mathrm{Zn}-\mathrm{O}$ vibrational mode & [7-11] \\
\hline 520 & 507 & 519 & & & 516 & & 502 & $\mathrm{v}_{4}$ phosphate mode & [12] \\
\hline \multirow[t]{2}{*}{551} & 553 & 551 & 557 & 559 & & 538 & 539 & V4 phosphate mode & {$[12]$} \\
\hline & & & & & & 595 & & $\mathrm{v}_{4}$ phosphate mode & {$[12]$} \\
\hline \multirow[t]{6}{*}{629} & 623 & 624 & 614 & 637 & 621 & 629 & 630 & V4 phosphate mode & [12] \\
\hline & 700 & & & 700 & 701 & & & $\mathrm{C}-\mathrm{H}$ bending & \\
\hline & & & & & & 682 & & $\mathrm{Si}-\mathrm{H}$ & {$[13]$} \\
\hline & & & & & & 775 & & O-C rocking & [14] \\
\hline & & & & & & 816 & & Si-O-Si bending & [15] \\
\hline & & & & & & 882 & & $\mathrm{Si}_{2} \mathrm{O}_{3}$ & {$[16,17]$} \\
\hline
\end{tabular}




\section{References - Electronic Supplementary Information}

1] Decremps, F.; Pellicer-Porres, J.; Saitta, A. M.; Chervin, J. C.; Polian, A. Highpressure raman spectroscopy study of wurtzite zno. Physical Review B 2002, 65, 0921011-0921014.

2] Gao, S. Y.; Zhang, H. J.; Wang, X. M.; Deng, R. P.; Sun, D. H.; Zheng, G. L. Znobased hollow microspheres: Biopolymer-assisted assemblies from zno nanorods. $J$. Phys. Chem. B 2006, 110, 15847-15852.

3] Wang, R. P.; Xu, G.; Jin, P. Size dependence of electron-phonon coupling in zno nanowires. Physical Review B 2004, 69, 1-4.

4] Munoz-Hernandez, G.; Escobedo-Morales, A.; Pal, U. Thermolytic growth of zno nanocrystals: Morphology control and optical properties. Crystal Growth \& Design 2009, 9, 297-300.

5] Verges, M. A.; Mifsud, A.; Serna, C. J. Formation of rod-like zinc-oxide microcrystals in homogeneous solutions. Journal of the Chemical Society-Faraday Transactions 1990, 86, 959-963.

6] Gupta, V.; Bhattacharya, P.; Yuzuk, Y. I.; Sreenivas, K.; Katiyar, R. S. Optical phonon modes in zno nanorods on si prepared by pulsed laser deposition. Journal of Crystal Growth 2006, 287, 39-43.

7] Farooqi, M. M. H.; Srivastava, R. K. Structural, optical and photoconductivity study of zno nanoparticles synthesized by annealing of zns nanoparticles. Journal of Alloys and Compounds 2017, 691, 275-286.

8] He, Y.; Sang, W. B.; Wang, J.; Wu, R. F.; Min, J. H. Polymer-assisted complexing controlled orientation growth of zno nanorods. Journal of Nanoparticle Research 2005, 7, 307-311.

9] Khan, M. F.; Ansari, A. H.; Hameedullah, M.; Ahmad, E.; Husain, F. M.; Zia, Q.; Baig, U.; Zaheer, M. R.; Alam, M. M.; Khan, A. M. et al. Sol-gel synthesis of thornlike zno nanoparticles endorsing mechanical stirring effect and their antimicrobial activities: Potential role as nano-antibiotics. Scientific Reports 2016, 6, 1-11.

10] Sahai, A.; Goswami, N. Structural and vibrational properties of zno nanoparticles synthesized by the chemical precipitation method. Physica E-Low-Dimensional Systems \& Nanostructures 2014, 58, 130-137. 
11] Vijayaprasath, G.; Murugan, R.; Hayakawa, Y.; Ravi, G. Optical and magnetic studies on gd doped zno nanoparticles synthesized by co-precipitation method. Journal of Luminescence 2016, 178, 375-383.

12] Pawlig, O.; Trettin, R. Synthesis and characterization of alpha-hopeite, zn-3(po4)(2)center dot 4h(2)o. Materials Research Bulletin 1999, 34, 1959-1966.

13] Tsai, C.; Li, K. H.; Sarathy, J.; Shih, S.; Campbell, J. C.; Hance, B. K.; White, J. M. Thermal treatment studies of the photoluminescence intensity of porous silicon. Applied Physics Letters 1991, 59, 2814-2816.

14] Babayan, S. E.; Jeong, J. Y.; Tu, V. J.; Park, J.; Selwyn, G. S.; Hicks, R. F. Deposition of silicon dioxide films with an atmospheric-pressure plasma jet. Plasma Sources Science and Technology 1998, 7, 286-288.

15] Xia, H.-L.; Tang, F.-Q. Surface synthesis of zinc oxide nanoparticles on silica spheres: Preparation and characterization. The Journal of Physical Chemistry B 2003, 107, 9175-9178.

16] Boyd, I. W.; Wilson, J. I. B. A study of thin silicon dioxide films using infrared absorption techniques. Journal of Applied Physics 1982, 53, 4166-4172.

17] Nakamura, M.; Mochizuki, Y.; Usami, K.; Itoh, Y.; Nozaki, T. Infrared absorption spectra and compositions of evaporated silicon oxides (siox). Solid State Communications 1984, 50, 1079-1081. 


\section{CHAPTER 4 CELLULAR TRANSCRIPTOME RESPONSES IN JURKAT LEUKEMIC AND PRIMARY CD4+ T CELLS FOLLOWING ZNO NANOPARTICLE TREATMENT}

\section{Introduction}

Cancer, known as a condition of uncontrolled cell differentiation, has major impact globally. In 2016, it was estimated that 1.6 million new cancer diagnoses would be made in the United States alone with approximately 600,000 mortalities [1]. For decades, preferred treatments have included chemotherapy, radiation and surgery [2]. Significant progress has been made in the past 25 years towards the development of effective chemotherapeutic agents $[2,3]$. Many challenges remain in controlling collateral damage to normal healthy cells while selectively killing cancerous cells, such as limiting acquired chemotherapy resistance in cancer cells and reducing undesirable side effects $[4,5]$. Metalbased nanoparticles (NPs) have emerged as promising drug-delivery vectors to improve drug solubility [4], increase drug payload at targeted tumor cells [6, 7], enhance bioimaging [8-11] and mediate hyperthermia-induced toxicity [12-14]. Although many NP systems are considered safe to biological systems [4], $\mathrm{nZnO}$ possesses an inherent natural toxicity related to its material properties [15-17]. As such, considerable attention in $\mathrm{nZnO}$ research over the past decade has focused on exploiting this inherent toxicity as a potential chemotherapeutic treatment. 
Several research studies in the past decade have investigated the potential selective toxicity of $\mathrm{nZnO}$ towards mammalian cancer cells when compared to their un-transformed primary cell counterparts including glioma, breast, prostate and immune system cell types [18-23]. Among these studies, zinc oxide NPs ( $\mathrm{ZnO} \mathrm{NP}), 6-8 \mathrm{~nm}$ in size, demonstrated a preferential toxicity $\left(\mathrm{IC}_{50}\right.$ of $\sim 0.17-0.21 \mathrm{mM}$ ) towards cancerous $\mathrm{T}$ cells $(28-35$ fold increase) while leaving normal quiescent $T$ cells essentially unaffected [18, 23]. In contrast, this NP-mediated selectivity towards immune system cancers appears to exceed ex vivo therapeutic indices $(<10)$ reported for chemotherapeutic agents, such as doxorubicin and cisplatin, commonly used for the treatment of acute myeloid leukemia and non-Hodgkin's lymphoma [24].

While multiple studies have demonstrated that nZnO NPs show preferential cytotoxicity towards cancer cells, the exact mechanism of the selectivity remains undetermined. Many potential hypotheses have been put forth to including both specific nanomaterial properties associated with the $\mathrm{nZnO}$ core or surface composition, and nonspecific interactions relating to NP size and surface area-to-volume ratios [25]. The surface structure of $\mathrm{nZnO}$ is strongly dependent upon the formation of an adsorbed neutral hydroxide layer which influences the surface charge of the $\mathrm{nZnO}$ at neutral $\mathrm{pH}$ and the formation of the biomolecular corona [26, 27]. $\mathrm{nZnO}$ has an isoelectric point at a $\mathrm{pH}$ of 9.5 which leads to a cationic surface potential at neutral $\mathrm{pH}$ which would increase electrostatic interactions between the $\mathrm{nZnO}$ and the anionic cellular membranes of cancer cells [28]. Additionally, the biomolecular corona formation partially determines the biological character of NP agglomerates, adhesion to cellular membranes and potential cellular uptake mechanisms [29-31]. Furthermore, these surface changes influence the 
solubility of $\mathrm{nZnO}$ agglomerates in both the extracellular environment and intracellular compartments. Internalized extracellular zinc or the release of soluble zinc ions within the cell contribute to signaling cascades that may contribute to the toxic response [22, 32]. Additionally, it has been shown that $\mathrm{nZnO}$ undergoes rapid preferential dissolution in acidic cancer microenvironment leading to oxidative stress, mitochondrial damage and cell cycle arrest proceeding cell death [22].

In addition to the differential effects imparted by $\mathrm{nZnO}$ material properties, specific cellular mechanisms have also been suggested as contributing factors in this selective toxicity. One proposed cellular mechanism implicates the differential reactive oxygen species (ROS) production observed in proliferating cancerous cells compared to primary cells $[18,19,33,34]$. Since cancerous cells are known to express higher amounts of ROS and ROS-associated signaling proteins due to their increased metabolism [35], exposure to low concentrations of $\mathrm{nZnO}$ significantly increases cellular ROS production inducing rapid cell death [32]. On the other hand, in normal cells basal levels of ROS are much lower with fewer available signaling molecules available to ramp up ROS production in response to external stressors. Thus, the level of ROS induced by $\mathrm{nZnO}$ treatment in primary cells may be insufficient to cause cell death [35].

The goal of this study was to investigate whether the preferential $\mathrm{nZnO}$ toxicity observed in Jurkat leukemic and primary $\mathrm{CD}^{+} \mathrm{T}$ cells results from differential transcriptional regulation of cellular processes. Since zinc is a necessary component of many critical cellular processes, excess zinc could have deleterious effects on cellular function. Towards this end, the gene expression of Jurkat leukemic and primary $\mathrm{CD} 4^{+} \mathrm{T}$ cells was evaluated using Illumina's Human HT-12 BeadChip arrays microarray to 
determine how $\mathrm{nZnO}$ treatment differentially modulates the transcriptome in a manner that could explain the discordant cytotoxicity observed between these cells. Using Ingenuity Pathway Analysis (IPA), differential gene and signaling pathway regulation were evaluated to discern possible transcriptome contributions to the differential toxicity. Microarray platforms facilitate the analysis of numerous genes simultaneously and the identification of potential transcriptional regulators of NP-induced toxicity. To date, our results have identified several key groups of gene transcripts, including proteins responsible for zinc sequestration and transport, oxidative stress and cell death signaling molecules, inflammatory and stress response cytokines, metabolism proteins, and cell cycle regulators that may be potentially involved in the cancer cell selectivity. Future studies are needed to validate these preliminary findings including reverse transcription polymerase chain reaction (RT-PCR) to verify mRNA expression levels, western blotting to assess proteome changes, and ultimately studies involving gain or loss of function.

\section{Materials and Methods}

\section{Zinc Oxide Synthesis and Characterization}

$\mathrm{nZnO}$ was fabricated using forced hydrolysis method. Briefly, a zinc acetate precursor was added to a DEG solvent and the resulting mixture was heated while stirring to approximately $80{ }^{\circ} \mathrm{C}$. A desired amount of nanopure water was introduced and the temperature increased to $150{ }^{\circ} \mathrm{C}$ for 90 minutes. The resulting colloid was then cooled to room temperature and the NPs collected through centrifugation at 21,000 rpm, washed with ethanol, and the resulting precipitate dried overnight at $60{ }^{\circ} \mathrm{C}$. Prior to use in downstream biological assays, the NPs were thoroughly characterized. A Rigaku Miniflex 600 X-ray diffractometer using Rigaku PDXL analysis software version 1.8.0.3 was employed to 
collect XRD spectra and determine crystal structure and phase, lattice parameters, and average crystalline size. Average particle size and morphology were obtained using a JEOL JEM-2100 HR analytical transmission electron microscope. FTIR using a Bruker Tensor 27 spectrometer was used to evaluate surface structure and room-temperature optical spectra in the ultraviolet and visible light wavelengths were collected using a CARY 5000 spectrophotometer to evaluate band gap and NP sedimentation. A Malvern Zetasizer NanoZS was used to determine $\zeta$-potential and hydrodynamic size, respectively. To determine the isoelectric point (IEP), zeta potentials of the $\mathrm{nZnO}$ were measured in nanopure water as a function of $\mathrm{pH}$. The $\mathrm{pH}$ of the room temperature NP dispersion was varied from a $\mathrm{pH}$ of 6 to a $\mathrm{pH}$ value of 12 using $1.0 \mathrm{~N} \mathrm{HCl}$ and $1.0 \mathrm{~N} \mathrm{NaOH}$ prior to collecting the data. At least six data collections per run were performed on three separate aliquots of the $\mathrm{ZnO}$ suspension for each sample.

\section{Extracellular Dissolution}

For extracellular dissolution experiments, $\mathrm{nZnO}$ stock dispersions were prepared as previously described at $6 \mathrm{mM}$ concentrations. To simulate cellular assay conditions, an aliquot of each stock dispersion was added to cellular media at a final $\mathrm{nZnO}$ concentration of $0.6 \mathrm{mM}$. All dispersions were stirred continuously to prevent $\mathrm{nZnO}$ sedimentation. At desired time points, an aliquot of the sample was removed and centrifuged at 5,000 $\mathrm{xg}$ for 20 minutes using an Amicon ${ }^{\mathrm{TM}}$ Ultra-4 Centrifugal Filter Unit with a 3-Kd molecular weight cutoff $(0.1 \mathrm{~nm}$ pore size $)$ to remove any undissolved $\mathrm{nZnO}$ while allowing free zinc ions to pass through. Quantitative analysis of the dissolved $\mathrm{Zn}^{2+}$ ions was conducted on a Thermo X Series 2 quadrupole inductively-coupled plasma mass spectrometer (ICP-MS) under normal operating conditions (i.e., no CCT) with the $X_{t}$ cone set. Instrument 
performance was evaluated and optimized for each run. The instrument was calibrated using multi-element calibration standards containing $\mathrm{Zn}, \mathrm{Cr}, \mathrm{Mn}, \mathrm{Fe}, \mathrm{Co}$ and $\mathrm{Ni}$ in $2 \%$ $\mathrm{HNO}_{3}$ at concentrations of approximately 1, 10, 100 and $1000 \mathrm{ppb}$. Instrument drift was monitored and corrected using $20 \mathrm{ppb}$ indium as an internal standard introduced online.

\section{$\underline{\text { Cell Culture and Toxicity Experiments }}$}

For this study, Jurkat leukemic and primary $\mathrm{CD}^{+} \mathrm{T}$ cell line were employed. Jurkat leukemic cells (ATCC, Rockville, MD) were cultured in RPMI 1640 containing 10 mM HEPES and supplemented with $10 \% \mathrm{FBS}, 1 \%$ penicillin/streptomycin, and $2 \mathrm{mM} \mathrm{L}-$ glutamine per manufacturer's recommendations. Cells were maintained in log phase and seeded at a concentration of $5 \times 10^{5}$ cells/mL in a 96 -well plate for viability assays. The $\mathrm{nZnO}$ solutions were created by suspending the NPs in autoclaved nanopure water and sonicated for 30 minutes using a power of $1.05 \mathrm{~W}$ to deliver a determined sonication energy [36] of $181 \mathrm{~J} / \mathrm{mL}$ to obtain stable $\mathrm{nZnO}$ dispersions [37]. Working stock dilutions were prepared by introducing the NP stock to cellular media and vortex mixing for 30 seconds. The NPs were then added to cells $\left(5 \times 10^{5}\right.$ cells $\left./ \mathrm{mL}\right)$ and cultured for 6 and 24 hours at 37 ${ }^{\circ} \mathrm{C}$ and $5 \% \mathrm{CO}_{2}$. For the alamar blue metabolic assay, $10 \% \mathrm{v} / \mathrm{v}$ alamar Blue was introduced to cells 4 hours prior to the desired endpoint (e.g. at 20 hours after treatment with NPs for 24-hour assay) and incubated at $37{ }^{\circ} \mathrm{C}$ and $5 \% \mathrm{CO}_{2}$. The fluorescence intensity was then determined at 24 hours by a Biotek Synergy MX plate reader using excitation/emission at $530 / 590 \mathrm{~nm}$.

For $\mathrm{CD}^{+} \mathrm{T}$ cell viability experiments, written informed consent was obtained from all blood donors and the University Institutional Review Board approved this study. PBMCs (peripheral blood mononuclear cells) were obtained from healthy human blood 
samples via Ficoll-Hypaque density centrifugation (Histopaque-1077, Sigma, St Louis, $\mathrm{MO}$ ), and $\mathrm{CD} 4^{+} \mathrm{T}$ cells isolated by negative immunomagnetic selection [18]. The purified $\mathrm{CD}^{+} \mathrm{T}$ cells (>95\% purity) were cultured in RPMI medium with $10 \%$ FBS at a concentration of $1 \times 10^{6}$ cells $/ \mathrm{ml}$. CD4 ${ }^{+} \mathrm{T}$ cells were then incubated for 6 and 24 hours with $\mathrm{nZnO}$ dispersions prepared as described above to establish an $\mathrm{IC}_{50}$. $\mathrm{CD}^{+} \mathrm{T}$ cells were then stained and viability analyzed by flow cytometry. After the treatment period, cells were washed with PBS and resuspended in FACS buffer (PBS/15 \% FBS/0.02 \% $\mathrm{NaN}_{3}$ ) and stained with a FITC labeled anti-HLA ABC antibody (BD Biosciences, San Jose, CA). Cells were subsequently washed, and stained propidium iodide (PI) to detect dead cells using a BD FACS Calibur flow cytometer.

\section{$\underline{\text { RNA Isolation }}$}

Total RNA was extracted from $\mathrm{nZnO}$ treated Jurkat leukemic and primary $\mathrm{CD} 4^{+} \mathrm{T}$ cells using the RNeasy Kit (Qiagen, Inc, Valencia, CA) according to the manufacturer's protocol. Bleach gels and a Biotek Synergy $\mathrm{MX}^{\circledR}$ plate reader fitted with a micro-volume analysis plate were employed to ensure the resulting RNA was free of contaminating proteins and genomic DNA. Bleach gels were prepared according to the protocol published by Arlanda et al. (2012) which involves the addition of $1.0 \%$ v/v Clorox ${ }^{\circledR}$ bleach (e.g. 0.5 $\mathrm{mL}$ in $50 \mathrm{~mL}$ ) into a $1.0 \%$ w/v agarose solution in $1 \mathrm{x}$ TAE buffer (e.g. $0.5 \mathrm{~g}$ in $50 \mathrm{~mL}$ ) [38]. The resulting mixture was heated in a microwave until the agarose had melted and cooled prior to the addition of ethidium bromide to a final concentration of $0.5 \mu \mathrm{g} / \mathrm{mL}$. Solidified gels were submerged with 1 x TAE buffer and loaded with $1 \mu \mathrm{g}$ total RNA with a $1 \times$ DNA loading buffer. The gels were run for 30 minutes at $100 \mathrm{~V}$ prior to imaging under UV transillumination. Samples were considered acceptable if the bleach gels showed 
only minimal traces of genomic DNA and the absorbance ratio A260/A280 fell between 1.8 - 2.0. Additional testing was performed at the National Institute for Occupational Safety and Health (NIOSH) in Morgantown, WV using an Agilent 2100 Bioanalyzer (Agilent Technologies, Palo Alto, CA) to confirm RNA integrity (RIN >8) and concentration. Acceptable samples were then combined to reduce biological variation and increase the total amount of mRNA.

Microarray Analysis of Global Gene Expression Profile

The following analysis was performed at the National Institute for Occupational Safety and Health (NIOSH) in Morgantown, WV. The global gene expression profile for the isolated Jurkat and CD4 ${ }^{+}$RNA was obtained using Human HT-12_v3_BeadChip arrays (Illumina, Inc, San Diego, CA) and experiments conducted in compliance with Minimal Information About a Microarray Experiment (MIAME) protocols. An Illumina Totalprep RNA Amplification Kit (Ambion, Inc.) was used to obtain biotin labeled cDNA followed by Chip hybridizations, washings and Cy3-streptavidin staining. The Beadstation 500 platform (Illumina, Inc) was employed for chip scanning according to the manufacturer's protocol. Prior to loading into the Beadstudio (Framework version 3.0.19.0) Gene Expression module v.3.0.14, metric files confirmed that the samples fluoresced at comparable levels. Proper chip detection was verified using housekeeping, hybridization control, stringency, and negative control genes. Illumina BeadArray expression data were exported into flat files comprised of mean fluorescent intensity across like beads and bead variance estimates and analyzed in Bioconductor. The microarray array was processed using the 'lumi' Bioconductor package to obtain normalized and annotated expression data followed by 'limma' package analysis to obtain FDR p values and log fold changes [39]. 
Significantly differentially expressed genes (SDEG), to be used for subsequent analysis, were selected based on fold change values $(\mathrm{FC})>1.5$ and false discovery rate (FDR) $\mathrm{p}$ values $<0.05$. Ingenuity Pathway Analysis (IPA, Ingenuity Systems, www.ingenuity.com) was used for subsequent bioinformatics analysis.

\section{Results}

$\underline{\text { nZnO Characterization }}$

$\underline{\text { Size, Morphology and Composition }}$

Zinc oxide NPs were prepared using a forced hydrolysis method that has known selective toxicity towards Jurkat leukemic cells when compared to primary $\mathrm{CD} 4^{+} \mathrm{T}$ cells $[18,23]$. Prior to use in downstream biologicals assays, the $\mathrm{nZnO}$ was thoroughly characterized. X-ray diffraction revealed that the $\mathrm{nZnO}$ contained a wurtzite crystal structure with no identifiable phase impurities (Figure 4.1A). Transmission electron microscopy (TEM) confirmed the expected spherical morphology of the nZnO (Figure 4.1B). Additionally, size measurements from the resulting TEM images demonstrated that the average NP to be $9.7 \pm 0.3 \mathrm{~nm}$. The atomic composition of the nanomaterial powder was analyzed using x-ray photoelectron spectroscopy (XPS) and identified zinc, oxygen and carbon species (Figure 4.1C).

\section{$\underline{\text { Surface Structure Analysis }}$}

The forced hydrolysis method employed to synthesize the $\mathrm{nZnO}$ used in this study is known to deposit surface-adsorbed functional groups, related to the synthesis components, onto the NP surface $[37,40]$. FTIR was utilized to identify these surfaceadsorbed components (Figure 4.2A). Evidence of the synthesis precursor (zinc acetate) remnants was observed with the presence of the $v_{s}\left(\mathrm{COO}^{-}\right)$and $v_{a s}\left(\mathrm{COO}^{-}\right)$carboxylic acid 
vibrational modes at $1412 \mathrm{~cm}^{-1}$ and $1591 \mathrm{~cm}^{-1}$, respectively [41-43]. The O-H bending mode at $910 \mathrm{~cm}^{-1}$ and the $v(\mathrm{C}-\mathrm{OH})$ mode at $1076 \mathrm{~cm}^{-1}$ at $[42,43]$ could also be secondary to the synthesis precursor or molecular fragments remaining from the DEG reaction solvent [40]. Additionally, the DEG sample displayed a broad FTIR peak associated with surface adsorbed $\mathrm{OH}$ groups at $3410 \mathrm{~cm}^{-1}$ [41-44].

Surface-adsorbed molecules plus intrinsic surface defects influence the charge density and ionization potential of the $\mathrm{nZnO}$. These changes result in augmented surface charge which can be evaluated through electrophoretic mobility measurement [40]. The isoelectric point of nanomaterials refers to the $\mathrm{pH}$ at which the $\zeta$-potential value is equal to zero. Figure 4.2B demonstrates the $\mathrm{pH}$ dependent changes in the $\zeta$-potential of the $\mathrm{nZnO}$. The established IEP for this sample is $\sim 9.5$ and is consistent with $\mathrm{nZnO}$ made with this synthesis method $[37,40]$. Below this point, the $\zeta$-potential values trend towards positive values with a maximum value of $39.3 \pm 0.7$ observed at a $\mathrm{pH}$ of 8.0. At $\mathrm{pH}$ values above the IEP, negative $\zeta$-potential values were measured. This characteristic IEP plot results from the surface-adsorbed neutral hydroxyl groups that form $\mathrm{M}-\mathrm{OH}$ surface layers, as can be seen in FTIR data (Figure 4.2A). Above the IEP (higher pH values), chemisorbed protons $\left(\mathrm{H}^{+}\right)$migrate from the NP surface into the aqueous medium resulting in partially bonded oxygen atoms $\left(\mathrm{M}-\mathrm{O}^{-}\right)$and the observed negative $\zeta$-potential measurements [26, 40]. In more the acidic conditions below the IEP, aqueous protons migrate to the $\mathrm{nZnO}$ surface to form a surface adsorbed water layer $\left(\mathrm{M}-\mathrm{OH}_{2}{ }^{+}\right)$groups and a positively charged surface layer $[26,40]$. 


\section{$\underline{\text { Agglomeration Behavior and Dissolution Potential }}$}

$\mathrm{ZnO}$ NPs are known to exhibit strong agglomeration behavior in a variety of biologically relevant solutions [37]. Therefore, an important consideration in nZnO toxicity studies is the stability of the treatment dispersions during the evaluation time frame. To access this stability, FBS/PBS nZnO stock dispersion were introduced into RPMI-based cellular media at a concentration of $486 \mu \mathrm{g} / \mathrm{mL}$. The hydrodynamic diameters of the suspended agglomerates were measured at regular intervals over a 24-time frame via dynamic light scattering (DLS). Figure 4.3A illustrates the hydrodynamic size distributions at several time periods. The initial size distribution (0 hours) demonstrates that the dispersion is composed of agglomerates primarily centered around $300 \mathrm{~nm}$. While most of the agglomerates are narrowly distributed from $\sim 200-475 \mathrm{~nm}$, there is a small percentage of agglomerates between $10-40 \mathrm{~nm}$. At later time points (6 and 12 hours), the overall size distribution remained centered at $\sim 350 \mathrm{~nm}$ although had broadened indicating the existence of larger agglomerates in dispersion. This broadening distribution trend continued through the remainder of the evaluation period. These results indicate that the agglomeration behavior of the $\mathrm{nZnO}$ dispersion is somewhat variable with increasing time, however; a large percentage of particles persist within a stable hydrodynamic range during the several hours it takes to complete the assay.

Another critical material property of $\mathrm{nZnO}$ is its solubility in many solution systems [45]. The ionic zinc released from the $\mathrm{nZnO}$ is considered a primary mechanism in NPinduced toxicity. Ionic zinc levels for $\mathrm{nZnO}$ dispersions utilized in the cellular toxicity assays were evaluated using ICP-MS at several time points over 48 hours (Figure 4.3B). A 
time-dependent linear increase in released $\mathrm{Zn}^{2+}$ was observed over 48 hours with ionic zinc values approaching $\sim 20 \mu \mathrm{g} / \mathrm{mL}$ of $\mathrm{Zn}^{2+}$ or roughly $5 \%$ of the initial concentration.

\section{$\underline{\text { Effects of } \mathrm{nZnO} \text { on Jurkat Leukemic and Primary CD4 }{ }^{+} \text {Cell Transcriptomes }}$}

Based on previous data, $\mathrm{nZnO}$ is known to exhibit preferentially toxicity towards Jurkat leukemic $\mathrm{T}$ cells when compared to primary $\mathrm{CD}^{+} \mathrm{T}$ cells $[18,23,34]$. Several hypotheses have been put forth to explain this selective toxicity including increased oxidative stress, proliferation, or dissolution in the acidic environment of cancerous cells $[18,20,22,46]$. However, no definitive mechanisms have been identified thus far. To address this question, genome-wide transcriptional profiling was employed at 6 hours to potentially identify early to intermediate transcriptional changes caused by $\mathrm{nZnO}$ treatment. Several $\mathrm{nZnO}$ concentrations were chosen including those above and below the $\mathrm{IC}_{25}$ at 6 hours and the $\mathrm{IC}_{50}$ at 24 hours for both cell types. Importantly, the $\mathrm{IC}_{50}$ at 24 hours and the $\mathrm{IC}_{25}$ at 6 hours were found to be nearly identical for both cell types, which helps to validate the concentration ranges used in this study (Figure 4.4A and B). In this study, mRNA was isolated at 6 hours post nZnO treatment to identify genes that are differentially expressed at a relatively early time point that precedes the considerable cell death observed at $24 \mathrm{~h}$. Thus, $\mathrm{nZnO}$ concentrations of $6.1,12.2,24.4,40.7$, and $61.0 \mu \mathrm{g} / \mathrm{mL}$ were used for Jurkat leukemic cells, and 61.0, 122.0, 244.0, and 407.0 $\mu \mathrm{g} / \mathrm{mL}$ used for primary CD4 ${ }^{+} \mathrm{T}^{-}$ cells. The lowest $\mathrm{nZnO}$ concentration used in each cell group produced little to no toxicity at 24 hours, while the highest dose caused considerable toxicity. Expression BeadChips were used to determine global expression levels for three replicate experiments for all treatment conditions. 
Initial analysis focused on general trends observed within the transcriptome analysis. Limma statistical analysis in Bioconductor [39] was employed to determine the significantly differentially expressed genes (SDEGs) following nZnO exposure. Fold change values were determined by comparing the normalized fluorescent data to the control treatment group (no nZnO). FDR p-values were determined by evaluating the spot signal intensity to the signal intensity of background or non-specific gene binding microarray spots. Genes that had fold change values of at least 1.5 and FDR p-values less than 0.05 were chosen for further analysis (Figure 4.3C). The number of SDEGs for both cell types increased in a dose-dependent manner. Primary $\mathrm{CD}^{+} \mathrm{T}$ cells showed significantly more differentially expressed genes compared to cancerous Jurkat cells (Figure 4.3C). The number of differentially expressed genes at the lowest $\mathrm{nZnO}$ concentration evaluated in each cell type $\left(6.1\right.$ and $61.0 \mu \mathrm{g} / \mathrm{mL}$ for the Jurkat and CD4 ${ }^{+} \mathrm{T}$ cells, respectively) was 12 for Jurkat cells and 1,041 for the primary CD4 ${ }^{+} \mathrm{T}$ cells. At the highest NP concentrations $\left(61.0\right.$ and $407.0 \mu \mathrm{g} / \mathrm{mL}$ for the Jurkat and CD4 ${ }^{+} \mathrm{T}$ cells, respectively), the number of SDEGs was 781 for Jurkat cells and 2,161 for primary T cells. As the concentration of NPs increased beyond the $\mathrm{IC}_{25}$ in the Jurkat cells, the number of SDEGs did not increase appreciably; however, the number of expressed genes continued to increase incrementally through the evaluated concentration range.

An analysis of the up- and down-regulated genes revealed a similar dose-dependent pattern for the total number of expressed genes (Figure 4.4D). In general, there are no significant differences in the relative number of up-regulated versus down-regulated genes for any one NP concentration or cell type. For the Jurkat cells, the lower nZnO concentrations $(6.1$ and $12.2 \mu \mathrm{g} / \mathrm{mL})$ had more up-regulated genes when compared to 
concentrations at or above the $\operatorname{IC}_{50}$ value $(24.4,40.7$ and $61.0 \mu \mathrm{g} / \mathrm{mL})$. In the primary cells, more down-regulated genes were observed for the 244.0 and $407.0 \mu \mathrm{g} / \mathrm{mL}$ NP treatments.

The Ingenuity Pathway Analysis (IPA) knowledgebase was used to classify the expressed genes into functional classes (Table 4.1) It is noteworthy that in primary cells the number of SDEGs in several of the functional classes (i.e., enzymes, G-protein coupled receptors, ion channels, phosphatases, transcriptional and translational regulators, and genes belonging to the other category) is roughly double that that in Jurkat cells. There were also substantially more cytokine and enzyme genes differentially expressed in primary cells. The functional class with the most SDEGs was designated as 'other' and includes metallothioneins and other zinc binding proteins, and zinc transporters. Transcriptional regulators were also enriched within both cells types as were transcripts encoding enzymes, kinases and phosphatases.

\section{$\underline{\text { Metallothioneins }}$}

Considering the role that metallothioneins (MT) have in buffering intracellular zinc, numerous studies have investigated the MT transcriptome in response to sub-lethal [47-53] and lethal concentrations [54-57] of zinc. Even at low doses of zinc or nZnO, metallothionein expression is upregulated. Among the differentially expressed genes in Jurkat and primary cells, 11 MT genes were identified (Figure 4.4). As a heterogeneous family of proteins, MTs contain several isoforms and sub-isoforms which differ in structure, cellular location and, to a limited extent, function $[58,59]$. MTF1 is the most widely studied metallothionein. It responds to small changes in intracellular zinc and upon zinc binding, travels to the nucleus where it acts as a transcription factor for other MT genes [58]. Of the major gene isoforms, the MT-1 and MT-2 families, are widely 
distributed throughout mammalian tissues where they contribute to copper and zinc regulation, heavy metal detoxification, immune system function, digestive tract functions, and cell transcription [59]. A minor isoform is MT-3 which is primarily localized to the central nervous system,[59], and research on this isoform is sparse. In our data set the MT1 gene sub-isoforms (MTF1, MT1A, MT1E, MT1G, MT1H, MT1M, MT1JP and MT1X) were the most prominently upregulated MT genes for both cell types, however the degree of upregulation was significantly higher in primary T cells, especially for MT1G, MT1H and MT1M (Figure 4.4). While some of the individual sub-isoforms have recently been linked to certain disease states, definite explanations for the variable differentiation of these MT-1 sub-isoforms is lacking [60]. Additionally, MT2A is upregulated in both cell types and increased MT3 expression was observed exclusively in primary cells.

\section{$\underline{\text { Solute-Linked Carrier Proteins }}$}

The solute-linked carrier family of proteins is a rather large and diverse group of proteins responsible for the transport and regulation of multiple substances essential for normal cell function. Many of these proteins transport xenobiotics and various drugs making them promising targets for disease remediation $[61,62]$. Within the SLC proteins six families of genes regulate the transport of metal ions (SLC11, SLC30, SLC31, SLC39, SLC40, and SLC41) [62]. When Jurkat and primary CD4 ${ }^{+} \mathrm{T}$ cells were exposed to $\mathrm{nZnO}$, four of these families were differentially expressed (Figure 4.5). SLC3OA1 and SLC30A2 (commonly $\mathrm{ZnT} 1$ and $\mathrm{ZnT} 2$ ) are responsible for $\mathrm{Zn}^{2+}$ efflux from the cytosol to the extracellular spaces and into lysosomes, respectively [63]. Given their role in maintaining intracellular zinc steady state concentrations, it is not surprising that these genes were upregulated following $\mathrm{nZnO}$ treatment (Figure 4.5). SLC30A1, the most widely distributed 
ZnT protein, was upregulated in both cell types with expression levels in primary cells three or more times higher than in Jurkat cells. ZnT2, however, was only upregulated in the Jurkat cells.

In contrast to the $S L C 30$ transport family, the $S L C 39$ family, commonly referred to as Zips, controls zinc import into the cytosol of the cell [63, 64]. SLC39A1 or Zip1 was upregulated in all samples following $\mathrm{nZnO}$ treatment; however, SLC39A10 was downregulated. The upregulation of Zip1 is somewhat surprising as this would work to increase extracellular zinc levels; however, Zips are responsive to other signaling molecules such as hormones and cytokines [64]. Indeed, cytosolic zinc concentrations have been shown to increase substantially within minutes after $\mathrm{T}$ cell activation and the enhanced expression of numerous Zip proteins [65]. Therefore, it is possible that the expression of this transcript could be under the control of factors other than ionic zinc.

The ion transporters SLC11A2 and SLC4OA1 also have demonstrated ability to regulate zinc levels. SLC11A2 is a metal cation/proton exchange protein found in most human cell types [62]. SLC4OA1 is commonly known as a ferroportin in that its primary function is to maintain iron homeostasis. Iron, however, binds protein in multiple forms including oxo-diiron (Fe-O-Fe), oxo-iron-zinc (Fe-O-Zn), iron-sulfur clusters $(\mathrm{Fe}-\mathrm{S})$ and heme, thus playing a secondary role in zinc transport [66]. In primary cells, both transporters were downregulated. In the Jurkat cells, however, the expression of SLC11A2 is downregulated in concentrations at and below the $\mathrm{IC}_{50}$ but upregulated in the higher treatment conditions.

The last $S L C$ family that was differentially regulated by $\mathrm{nZnO}$ was the $S L C 31$ copper ion transporters [67]. While these transporters do not contribute to the control of 
zinc homeostasis, they are critical for the function of copper containing enzymes such as the ROS scavenger $\mathrm{Cu} / \mathrm{Zn}$ superoxide dismutase ( $\mathrm{Cu} / \mathrm{ZnSOD} 1)$ [67]. The upregulation of SLC31A1 and SLC31A2 observed in the primary $\mathrm{CD}^{+} \mathrm{T}$ cells could be associated with the oxidative stress experienced by the cells following nZnO treatment. SLC31A1 was also upregulated in Jurkat cells, however, SLC31A2 was downregulated.

Another group of differentially expressed transcripts belonged to SLC families responsible for the uptake of various sugar molecules. As quiescent cells, $\mathrm{CD}^{+}$ lymphocytes have very low energy requirements and utilize oxidative phosphorylation within the mitochondria to generate any needed ATP [68]. Upon activation, T cells dramatically increase energy stores by increasing both glucose intake and oxygen consumption. However, activated primary $\mathrm{T}$ cells and cancerous $\mathrm{T}$ cells prefer to use aerobic glycolysis following the upregulation of glucose transporters [68]. SLC2A1 or GLUT 1 is normally responsible for increases in glucose uptake in activated $\mathrm{T}$ cells; however, $S L C 2 A 3$, and $S L C 2 A 6$ also demonstrate differential regulation following $\mathrm{T}$ cell activation [69]. Following nZnO treatment, SLC2A1 was downregulated in both cell types. In contrast, $S L C 2 A 3, S L C 2 A 6$ and $S L C 2 A 14$ were upregulated (Figure 4.6). The remaining two glucose transporters, SLC45A4 and SLC50A1, were both downregulated in Jurkat cells. In primary cells, SLC45A4 demonstrated decreased expression, while SLC50A1 was upregulated. While the function of the $S L C 2 A 1$ is well characterized in immune system cells, little is known regarding the function of the remaining transcripts. Therefore, it is not possible to predict the net result of glucose uptake in $\mathrm{nZnO}$ treated Jurkat or $\mathrm{CD}^{+}{ }^{+}$cells.

The final group of $S L C$ families that were differentially expressed post $\mathrm{nZnO}$ treatment are responsible for the cellular transport of amino acids and small peptides. The 
first six of these transcripts (SLC1A4, SLC1A5, SLC3A2, SLC7A1, SLC7A5, and SLC7A11) were essentially upregulated in both cell types (Figure 4.8). However, the SLC1A5 transcript was downregulated at the lowest concentration of $\mathrm{nZnO}$ treatment in the Jurkat cells. The tripeptide glutathione (GSH) is an integral component of T cell activation with increases in GSH associated with T cell proliferation [70]. Since quiescent $\mathrm{T}$ cells have little need use for exogenous sources of cysteine (Cys) or cystine $\left(\mathrm{Cys}_{2}\right)$ needed for the enhanced GSH production, activated T cells require a means to obtain these amino acids [70]. The primary way by which activated $\mathrm{T}$ cells accomplish this is through the upregulation of the SLC1A4, SLC1A5, SLC3A2, and SLC7A11 genes. SLC1A4 and SLC1A5, also known as ASCT1 and ASCT2, respectively, transport cysteine, while SLC3A2 (CD98) and SLC7A11 comprise a cystine/glutamate antiporter [70]. Additionally, the glutamine importer $S L C 7 A$, and the $S L C 7 A 5$ (LAT1) leucine transporter are upregulated during TCR activation and are involved in lymphocyte metabolic reprograming for T cell differentiation $[71,72]$. The remaining two SDEGS in this $S L C$ family, SLC15A3, and SLC15A4, were significantly downregulated in the primary T cells, while only the SLC15A4 transcript was downregulated in the Jurkat cells (Figure 4.8). These small peptide transporters are known to assist in the cellular endocytosis of NOD1 and NOD2 ligands and signaling pathways involved in inflammation [73].

\section{Discussion}

The goal of this study was to identify potential genetic targets likely to account for the cancer cell selectivity of $\mathrm{nZnO}$. Towards this goal, Jurkat leukemic and CD4 ${ }^{+} \mathrm{T}$ cells were treated with varying concentrations of $\mathrm{nZnO}$ above and below the established $\mathrm{IC}_{50}$ (Figure 4.3B). At 6 hours post-treatment, mRNA was isolated from the treated cells, and a 
microarray was utilized to identify significantly differentiated changes in cellular transcription responses. IPA was then conducted to evaluate these changes in relationship to cell type and treatment concentration. From this analysis, several genes have been selected for further analysis.

Our initial analysis focused on a general overview of the transcriptome response and specific gene targets involved in the regulation of zinc homeostasis. Metallothioneins, responsible for maintaining zinc steady state conditions, were among the most highly upregulated genes identified (Figure 4.4). While these transcripts were upregulated in both cell types in a dose-dependent manner, their expression levels were significantly higher in the primary cells. Of these, MTF1 is of primary interest as it functions as a transcription factor for the MRE and ARE promoters responsible for the expression of all other MT proteins. The remaining MT SDEGs are also relevant as MT proteins sequester excess zinc and contribute to ROS remediation. Therefore, the increased expression of these genes, specifically in primary cells, may allow cells to remove excess zinc and reduce oxidative stress thereby preventing toxicity.

The other group of proteins involved in zinc homeostasis maintenance are those belonging to the SLC30 (ZnTs) and SLC39 (Zips) family of genes. Two genes, SCL30A1 and SLC 39A1 are of particular interest in this study. The SCL3OAl efflux transcript was significantly enhanced (up to 3 times) in primary cells over Jurkat cells. Since ZnT1 is localized primarily to the cellular membrane, increased expression of this protein would result in greater efflux of ionic zinc out of the cell. SLC39A1, or Zip1, functions as an importer of zinc into the cell which seems counterintuitive under conditions of excess zinc. However, this gene is important given that $\mathrm{T}$ cell activation results in the upregulation of 
Zip proteins. Additionally, the cation/proton exchange protein gene SLC11A2 is noteworthy as it was upregulated in primary cells yet regulated differently in Jurkat cells depending on $\mathrm{nZnO}$ concentration. Finally, the copper transporter gene SLC31A2 and its potential connection to the ROS scavenger $\mathrm{Cu} / \mathrm{Zn}$ superoxide dismutase $(\mathrm{Cu} / \mathrm{ZnSOD} 1)$ draws attention because of its potential contribution to oxidative stress remediation. This is especially relevant considering this transcript was upregulated in only the primary cells.

Two other types of differentially expressed transporters, sugar and amino acid transporters were also identified within the broad $S L C$ family of genes. The regulation of glucose/sugar transporters in $\mathrm{T}$ lymphocytes, are generally associated with $\mathrm{T}$ cell activation and increased cellular metabolism. Within our SDEGs, two sugar transporters were particularly noteworthy, SLC2A3, and SLC50A1. The expression levels for the SLC2A3 gene were much higher for the Jurkat cells than the primary cells which is counter to the expression patterns observed for the remaining glucose transporters. The SLC50Al gene, identified as a sugar efflux transport gene in plants and some mammals [74], was downregulated in the Jurkat cells yet upregulated in the primary cells. The differential regulation of the glucose transporters, especially in the $\mathrm{CD} 4^{+} \mathrm{T}$ cells, suggests that $\mathrm{nZnO}$ could potentially influence the activation state of T lymphocytes. Additional evidence of this was observed with the upregulation of numerous amino acid transporters (SLCIA4, SLC1A5, SLC3A2, SLC7A1, SLC7A5, and SLC7A11). During T cell activation, the amino acid demands of the $\mathrm{T}$ cells significantly increase to meet the proteomic needs of increased metabolism, cell survival, cellular proliferation and differentiation [75].

In addition to the above-mentioned genes, many other gene transcripts attracted attention and have been grouped within Table 4.2 into three broad categories, inflammatory 
mediators, T cell activation and cellular stress responses. Numerous research studies have investigated the ability of $\mathrm{nZnO}$ to affect immune activation in a variety of cells $[17,76-$ 78]. Indeed, cellular transcriptome and proteome changes post $\mathrm{nZnO}$ treatment have indicated that inflammatory responses may contribute to the toxic mechanism; however, the precise inflammatory mediators detected across the literature have not been consistent. Our initial analysis revealed several pro-inflammatory mediators (Table 4.2; Inflammatory Mediators) that were differentially expressed in primary cells indicating a potential role for these factors in the cytotoxicity. For example, the inflammatory genes included in Table 4.2 code for proteins involved in signaling pathways that promote $\mathrm{T}$ cell proliferation and survival [79].

Additional research has linked NP-induced cytotoxicity to cellular proliferation and the activation status of hematopoietic cells ([23] and unpublished data). Indeed, NP cytotoxicity has been linked to the proliferative capacity of the cell with the highest degree of toxicity associated with the most rapidly dividing cells (unpublished data). Considering this, it is important to ascertain if $\mathrm{nZnO}$ can influence the activation state of naïve primary T cells. Interestingly, several SDEGS relating to $\mathrm{T}$ cell activation were identified within our data set including upregulated transcripts for early activation markers such as CD69 (Table 4.2; T Cell Activation). Future studies are needed to verify that the observed SDEGS are associated with cellular activation responses.

Finally, Table 4.2 (Cellular Stress Responses) lists several gene transcripts associated with stress-induced proteins. Several of these genes are associated with heat shock proteins which facilitate the removal of misfolded proteins. Another interesting target is the HMOX1, or heme oxygenase 1 gene, which encodes an essential enzyme for 
heme catabolism, and had upregulated fold change values $\sim 50$ times higher in primary $\mathrm{T}$ cells than in Jurkat cells. It is noteworthy that HMOX1 deficient cells have demonstrated susceptibility to oxidative stress, and its upregulation is considered an adaptive mechanism that may protect cells from oxidative damage [80].

Although several of the above-mentioned transcriptional changes may relate to the selective toxicity mechanism of $\mathrm{nZnO}$, many additional studies are needed to confirm their potential significance. First, RT-PCR is needed to verify key results of the initial analysis. Secondly, western blot studies can confirm the actual protein expression levels of the SDEGs in question. Additionally, as many of these genes initiate signaling pathways that may contribute to the toxicity mechanism, evaluation of the expression and function of key downstream signaling proteins will be critical to the final analysis. Finally, gain or loss of function studies will be needed to confirm the functional significance of transcripts and mechanistic pathways found to be critical to the cancer cell selectivity of $\mathrm{nZnO}$. 


\section{References}

[1] Cancer statistics. https://www.cancer.gov/about-cancer/understanding/statistics (accessed October 24, 2017).

[2] Chabner, B. A.; Roberts, T. G. Timeline - chemotherapy and the war on cancer. Nature Reviews Cancer 2005, 5, 65-72.

[3] Ferrari, M. Cancer nanotechnology: Opportunities and challenges. Nat. Rev. Cancer 2005, 5, 161-171.

[4] Zhang, X.-Q.; Xu, X.; Lam, R.; Giljohann, D.; Ho, D.; Mirkin, C. A. Strategy for increasing drug solubility and efficacy through covalent attachment to polyvalent DNA-nanoparticle conjugates. ACS nano 2011, 5, 6962-6970.

[5] Marupudi, N. I.; Han, J. E.; Li, K. W.; Renard, V. M.; Tyler, B. M.; Brem, H. Paclitaxel: A review of adverse toxicities and novel delivery strategies. Expert Opinion on Drug Safety 2007, 6, 609-621.

[6] Qi, J. N.; Yao, P.; He, F.; Yu, C. L.; Huang, C. O. Nanoparticles with dextran/chitosan shell and bsa/chitosan core-doxorubicin loading and delivery. International Journal of Pharmaceutics 2010, 393, 176-184.

[7] Desai, N.; Trieu, V.; Yao, Z. Increased antitumor activity, intratumor paclitaxel concentrations, and endothelial cell transport of cremophor-free, albumin-bound paclitaxel, abi-007, compared with cremophor-based paclitaxel (vol 12, pg 1317, 2006). Clin. Cancer Res. 2006, 12, 3869-3869.

[8] Parveen, S.; Misra, R.; Sahoo, S. K. Nanoparticles: A boon to drug delivery, therapeutics, diagnostics and imaging. Nanomedicine 2012, 8, 147-166.

[9] Tasciotti, E.; Liu, X. W.; Bhavane, R.; Plant, K.; Leonard, A. D.; Price, B. K.; Cheng, M. M. C.; Decuzzi, P.; Tour, J. M.; Robertson, F. et al. Mesoporous silicon particles as a multistage delivery system for imaging and therapeutic applications. Nature Nanotechnology 2008, 3, 151-157.

[10] Xiong, H.-M. Zno nanoparticles applied to bioimaging and drug delivery. Advanced Materials 2013, 25, 5329-5335.

[11] Zvyagin, A. V.; Zhao, X.; Gierden, A.; Sanchez, W.; Ross, J. A.; Roberts, M. S. Imaging of zinc oxide nanoparticle penetration in human skin in vitro and in vivo. Journal of biomedical optics 2008, 13, 0640311-0640319. 
[12] Asin, L.; Ibarra, M. R.; Tres, A.; Goya, G. F. Controlled cell death by magnetic hyperthermia: Effects of exposure time, field amplitude, and nanoparticle concentration. Pharmaceutical Research 2012, 29, 1319-1327.

[13] Tomitaka, A.; Yamada, T.; Takemura, Y. Magnetic nanoparticle hyperthermia using pluronic-coated fe3o4 nanoparticles: An in vitro study. Journal of Nanomaterials 2012.

[14] Fujishima, A.; Cai, R. X.; Otsuki, J.; Hashimoto, K.; Itoh, K.; Yamashita, T.; Kubota, Y. Biochemical application of photoelectrochemistry - photokilling of malignant-cells with tio2 powder. Electrochimica Acta 1993, 38, 153-157.

[15] Zhang, H.; Ji, Z.; Xia, T.; Meng, H.; Low-Kam, C.; Liu, R.; Pokhrel, S.; Lin, S.; Wang, X.; Liao, Y.-P. et al. Use of metal oxide nanoparticle band gap to develop a predictive paradigm for oxidative stress and acute pulmonary inflammation. ACS Nano 2012, 6, 4349-4368.

[16] Sun, T.; Yan, Y.; Zhao, Y.; Guo, F.; Jiang, C. Copper oxide nanoparticles induce autophagic cell death in a549 cells. PloS one 2012, 7.

[17] Xia, T.; Kovochich, M.; Liong, M.; Mädler, L.; Gilbert, B.; Shi, H.; Yeh, J.; Zink, J.; Nel, A. Comparison of the mechanism of toxicity of zinc oxide and cerium oxide nanoparticles based on dissolution and oxidative stress properties. ACS nano $\mathbf{2 0 0 8 ,}$ 2, 2121-2134.

[18] Hanley, C.; Layne, J.; Punnoose, A.; Reddy, K. M.; Coombs, I.; Coombs, A.; Feris, K.; Wingett, D. Preferential killing of cancer cells and activated human t cells using zno nanoparticles. Nanotechnology 2008, 19, 1-10.

[19] Ostrovsky, S.; Kazimirsky, G.; Gedanken, A.; Brodie, C. Selective cytotoxic effect of zno nanoparticles on glioma cells. Nano Research 2009, 2, 882-890.

[20] Premanathan, M.; Karthikeyan, K.; Jeyasubramanian, K.; Manivannan, G. Selective toxicity of zno nanoparticles toward gram-positive bacteria and cancer cells by apoptosis through lipid peroxidation. Nanomedicine 2011, 7, 184-192.

[21] Sahu, D.; Kannan, G. M.; Tailang, M.; Vijayaraghavan, R. In vitro cytotoxicity of nanoparticles: A comparison between particle size and cell type. Journal of Nanoscience 2016, 2016, 1-9.

[22] Sasidharan, A.; Chandran, P.; Menon, D.; Raman, S.; Nair, S.; Koyakutty, M. Rapid dissolution of zno nanocrystals in acidic cancer microenvironment leading to preferential apoptosis. Nanoscale 2011, 3, 3657-3669. 
[23] Hanley, C.; Thurber, A.; Hanna, C.; Punnoose, A.; Zhang, J. H.; Wingett, D. G. The influences of cell type and zno nanoparticle size on immune cell cytotoxicity and cytokine induction. Nanoscale Research Letters 2009, 4, 1409-1420.

[24] Bosanquet, A.; Bell, P. Ex vivo therapeutic index by drug sensitivity assay using fresh human normal and tumor cells. Journal of experimental therapeutics \& oncology 2004, 4, 145-154.

[25] Nel, A.; Xia, T.; Mädler, L.; Li, N. Toxic potential of materials at the nanolevel. Science 2006, 311, 622-627.

[26] Qu, F.; Morais, P. C. An oxide semiconductor nanoparticle in an aqueous medium: A surface charge density investigation. The Journal of Physical Chemistry B 2000, 104, 5232-5236.

[27] Xu, M.; Li, J.; Iwai, H.; Mei, Q.; Fujita, D.; Su, H.; Chen, H.; Hanagata, N. Formation of nano-bio-complex as nanomaterials dispersed in a biological solution for understanding nanobiological interactions. Sci Rep 2012, 2, 1-6.

[28] Abercrombie, M.; Ambrose, E. J. The surface properties of cancer cells: A review. Cancer research 1962, 22, 525-548.

[29] Maiorano, G.; Sabella, S.; Sorce, B.; Brunetti, V.; Malvindi, M. A.; Cingolani, R.; Pompa, P. P. Effects of cell culture media on the dynamic formation of proteinnanoparticle complexes and influence on the cellular response. ACS Nano 2010, 4 , 7481-7491.

[30] Monopoli, M. P.; Aberg, C.; Salvati, A.; Dawson, K. A. Biomolecular coronas provide the biological identity of nanosized materials. Nature Nanotechnology 2012, 7, 779-786.

[31] Tenzer, S.; Docter, D.; Kuharev, J.; Musyanovych, A.; Fetz, V.; Hecht, R.; Schlenk, F.; Fischer, D.; Kiouptsi, K.; Reinhardt, C. et al. Rapid formation of plasma protein corona critically affects nanoparticle pathophysiology. Nature Nanotechnology 2013, 8, 772-781.

[32] Bisht, G.; Rayamajhi, S. Zno nanoparticles: A promising anticancer agent. Nanobiomedicine 2016, 3, 9.

[33] Akhtar, M. J.; Ahamed, M.; Kumar, S.; Khan, M. M.; Ahmad, J.; Alrokayan, S. A. Zinc oxide nanoparticles selectively induce apoptosis in human cancer cells through reactive oxygen species. Int J Nanomedicine 2012, 7, 845-857. 
[34] Thurber, A.; Wingett, D. G.; Rasmussen, J. W.; Layne, J.; Johnson, L.; Tenne, D. A.; Zhang, J. H.; Hanna, C. B.; Punnoose, A. Improving the selective cancer killing ability of zno nanoparticles using fe doping. Nanotoxicology 2012, 6, 440-452.

[35] Liou, G. Y.; Storz, P. Reactive oxygen species in cancer. Free Radic. Res. 2010, 44, 479-496.

[36] Taurozzi, J. S.; Hackley, V. A.; Wiesner, M. R. Ultrasonic dispersion of nanoparticles for environmental, health and safety assessment - issues and recommendations. Nanotoxicology 2011, 5, 711-729.

[37] Anders, C. B.; Chess, J. J.; Wingett, D. G.; Punnoose, A. Serum proteins enhance dispersion stability and influence the cytotoxicity and dosimetry of zno nanoparticles in suspension and adherent cancer cell models. Nanoscale Research Letters 2015, 10, 1-22.

[38] Aranda, P. S.; LaJoie, D. M.; Jorcyk, C. L. Bleach gel: A simple agarose gel for analyzing rna quality. Electrophoresis 2012, 33, 366-369.

[39] Gentleman, R. C.; Carey, V. J.; Bates, D. M.; Bolstad, B.; Dettling, M.; Dudoit, S.; Ellis, B.; Gautier, L.; Ge, Y. C.; Gentry, J. et al. Bioconductor: Open software development for computational biology and bioinformatics. Genome Biol. 2004, 5, $1-16$.

[40] Punnoose, A.; Dodge, K.; Rasmussen, J. W.; Chess, J.; Wingett, D.; Anders, C. Cytotoxicity of zno nanoparticles can be tailored by modifying their surface structure: A green chemistry approach for safer nanomaterials. Acs Sustainable Chemistry \& Engineering 2014, 2, 1666-1673.

[41] Farbun, I. A.; Romanova, I. V.; Terikovskaya, T. E.; Dzanashvili, D. I.; Kirillov, S. A. Complex formation in the course of synthesis of zinc oxide from citrate solutions. Russian Journal of Applied Chemistry 2007, 80, 1798-1803.

[42] Max, J. J.; Chapados, C. Infrared spectroscopy of aqueous carboxylic acids: Malic acid. Journal of Physical Chemistry A 2002, 106, 6452-6461.

[43] Max, J. J.; Chapados, C. Infrared spectroscopy of aqueous carboxylic acids: Comparison between different acids and their salts. Journal of Physical Chemistry A 2004, 108, 3324-3337.

[44] Xia, H.-L.; Tang, F.-Q. Surface synthesis of zinc oxide nanoparticles on silica spheres: Preparation and characterization. The Journal of Physical Chemistry B 2003, 107, 9175-9178. 
[45] Eixenberger, J.; Anders, C.; Hermann, R.; Brown, R.; Reddy, K. M.; Punnoose, A.; Wingett, D. Rapid dissolution of zno nanoparticles induced by biological buffers significantly impacts cytotoxicity. Chemical Research in Toxicology 2017, 30, 1641-1651.

[46] Taccola, L.; Raffa, V.; Riggio, C.; Vittorio, O.; Iorio, M. C.; Vanacore, R.; Pietrabissa, A.; Cuschieri, A. Zinc oxide nanoparticles as selective killers of proliferating cells. International Journal of Nanomedicine 2011, 6, 1129-1140.

[47] Allan, A. K.; Hawksworth, G. M.; Woodhouse, L. R.; Sutherland, B.; King, J. C.; Beattie, J. H. Lymphocyte metallothionein mrna responds to marginal zinc intake in human volunteers. British Journal of Nutrition 2000, 84, 747-756.

[48] Aydemir, T. B.; Cousins, R. J. Zinc supplementation of human subjects produces genomic-level responses. Faseb Journal 2006, 20, A627-A627.

[49] Cao, J. Y.; Cousins, R. J. Metallothionein mrna in monocytes and peripheral blood mononuclear cells and in cells from dried blood spots increases after zinc supplementation of men. Journal of Nutrition 2000, 130, 2180-2187.

[50] Chu, A. N.; Foster, M.; Ward, S.; Zaman, K.; Hancock, D.; Petocz, P.; Samman, S. Zinc-induced upregulation of metallothionein $(\mathrm{mt})-2 \mathrm{a}$ is predicted by gene expression of zinc transporters in healthy adults. Genes and Nutrition 2015, 10, 110.

[51] Hunt, J. R.; Beiseigel, J. M.; Johnson, L. K. Adaptation in human zinc absorption as influenced by dietary zinc and bioavailability. American Journal of Clinical Nutrition 2008, 87, 1336-1345.

[52] Ryu, M. S.; Langkamp-Henken, B.; Chang, S. M.; Shankar, M. N.; Cousins, R. J. Genomic analysis, cytokine expression, and microrna profiling reveal biomarkers of human dietary zinc depletion and homeostasis. Proc Natl Acad Sci U S A 2011, 108, 20970-20975.

[53] Sharif, R.; Thomas, P.; Zalewski, P.; Fenech, M. Zinc supplementation influences genomic stability biomarkers, antioxidant activity, and zinc transporter genes in an elderly australian population with low zinc status. Molecular Nutrition \& Food Research 2015, 59, 1200-1212.

[54] Lee, S. H.; Pie, J. E.; Kim, Y. R.; Lee, H. R.; Son, S. W.; Kim, M. K. Effects of zinc oxide nanoparticles on gene expression profile in human keratinocytes. Molecular \& Cellular Toxicology 2012, 8, 113-118. 
[55] Moos, P. J.; Olszewski, K.; Honeggar, M.; Cassidy, P.; Leachman, S.; Woessner, D.; Cutler, N. S.; Veranth, J. M. Responses of human cells to zno nanoparticles: A gene transcription study. Metallomics 2011, 3, 1199-1211.

[56] Triboulet, S.; Aude-Garcia, C.; Armand, L.; Gerdil, A.; Diemer, H.; Proamer, F.; Collin-Faure, V.; Habert, A.; Strub, J. M.; Hanau, D. et al. Analysis of cellular responses of macrophages to zinc ions and zinc oxide nanoparticles: A combined targeted and proteomic approach. Nanoscale 2014, 6, 6102-6114.

[57] Tuomela, S.; Autio, R.; Buerki-Thurnherr, T.; Arslan, O.; Kunzmann, A.; Andersson-Willman, B.; Wick, P.; Mathur, S.; Scheynius, A.; Krug, H. F. et al. Gene expression profiling of immune-competent human cells exposed to engineered zinc oxide or titanium dioxide nanoparticles. PloS one 2013, 8, 1-18.

[58] Sutherland, D. E.; Stillman, M. J. The "magic numbers" of metallothionein. Metallomics 2011, 3, 444-463.

[59] Thirumoorthy, N.; Shyam Sunder, A.; Manisenthil Kumar, K. T.; Senthil kumar, M.; Ganesh, G. N. K.; Chatterjee, M. A review of metallothionein isoforms and their role in pathophysiology. World Journal of Surgical Oncology 2011, 9, 54-54.

[60] Skutkova, H.; Babula, P.; Stiborova, M.; Eckschlager, T.; Trnkova, L.; Provaznik, I.; Hubalek, J.; Kizek, R.; Adam, V. Structure, polymorphisms and electrochemistry of mammalian metallothioneins-a review. Int. J. Electrochem. Sci 2012, 7, 12415-12431.

[61] Lin, L.; Yee, S. W.; Kim, R. B.; Giacomini, K. M. Slc transporters as therapeutic targets: Emerging opportunities. Nature reviews. Drug discovery 2015, 14, 543560.

[62] Alexander, S. P. H.; Benson, H. E.; Faccenda, E.; Pawson, A. J.; Sharman, J. L.; Spedding, M.; Peters, J. A.; Harmar, A. J.; Collaborators, C. The concise guide to pharmacology 2013/14: Transporters. British Journal of Pharmacology 2013, 170, 1706-1796.

[63] Liuzzi, J. P.; Cousins, R. J. Mammalian zinc transporters. Annu. Rev. Nutr. 2004, 24, 151-172.

[64] Jeong, J.; Eide, D. J. The slc39 family of zinc transporters. Molecular Aspects of Medicine 2013, 34, 612-619.

[65] Yu, M. C.; Lee, W. W.; Tomar, D.; Pryshchep, S.; Czesnikiewicz-Guzik, M.; Lamar, D. L.; Li, G. J.; Singh, K.; Tian, L.; Weyand, C. M. et al. Regulation of t 
cell receptor signaling by activation-induced zinc influx. Journal of Experimental Medicine 2011, 208, 775-785.

[66] Ward, D.; Kaplan, J. Ferroportin-mediated iron transport: Expression and regulation. Biochimica et biophysica acta 2012, 1823, 1426-1433.

[67] Kim, H.; Wu, X. B.; Lee, J. Slc31 (ctr) family of copper transporters in health and disease. Molecular Aspects of Medicine 2013, 34, 561-570.

[68] MacIver, N. J.; Jacobs, S. R.; Wieman, H. L.; Wofford, J. A.; Coloff, J. L.; Rathmell, J. C. Glucose metabolism in lymphocytes is a regulated process with significant effects on immune cell function and survival. Journal of Leukocyte Biology 2008, 84, 949-957.

[69] Macintyre, A. N.; Gerriets, V. A.; Nichols, A. G.; Michalek, R. D.; Rudolph, M. C.; Deoliveira, D.; Anderson, S. M.; Abel, E. D.; Chen, B. J.; Hale, L. P. et al. The glucose transporter glut1 is selectively essential for $\mathrm{cd} 4 \mathrm{t}$ cell activation and effector function. Cell metabolism 2014, 20, 61-72.

[70] Levring, T. B.; Hansen, A. K.; Nielsen, B. L.; Kongsbak, M.; von Essen, M. R.; Woetmann, A.; Ødum, N.; Bonefeld, C. M.; Geisler, C. Activated human cd4+ t cells express transporters for both cysteine and cystine. 2012, 2, 1-6.

[71] Sinclair, L. V.; Rolf, J.; Emslie, E.; Shi, Y.-B.; Taylor, P. M.; Cantrell, D. A. Antigen receptor control of amino acid transport coordinates the metabolic reprogramming that is essential for $\mathrm{t}$ cell differentiation. Nature immunology 2013, $14,500-508$.

[72] Wang, R.; Dillon, Christopher P.; Shi, Lewis Z.; Milasta, S.; Carter, R.; Finkelstein, D.; McCormick, Laura L.; Fitzgerald, P.; Chi, H.; Munger, J. et al. The transcription factor myc controls metabolic reprogramming upon t lymphocyte activation. Immunity 2011, 35, 871-882.

[73] Saxena, M.; Yeretssian, G. Nod-like receptors: Master regulators of inflammation and cancer. Frontiers in Immunology 2014, 5, 1-16.

[74] Chen, L. Q.; Hou, B. H.; Lalonde, S.; Takanaga, H.; Hartung, M. L.; Qu, X. Q.; Guo, W. J.; Kim, J. G.; Underwood, W.; Chaudhuri, B. et al. Sugar transporters for intercellular exchange and nutrition of pathogens. Nature 2010, 468, 527-U199.

[75] Ren, W.; Liu, G.; Yin, J.; Tan, B.; Wu, G.; Bazer, F. W.; Peng, Y.; Yin, Y. Aminoacid transporters in t-cell activation and differentiation. Cell Death \& Disease 2017, $8,1-9$. 
[76] Saptarshi, S. R.; Duschl, A.; Lopata, A. L. Biological reactivity of zinc oxide nanoparticles with mammalian test systems: An overview. Nanomedicine 2015, 10, 2075-2092.

[77] Saptarshi, S. R.; Feltis, B. N.; Wright, P. F. A.; Lopata, A. L. Investigating the immunomodulatory nature of zinc oxide nanoparticles at sub-cytotoxic levels in vitro and after intranasal instillation in vivo. Journal of Nanobiotechnology 2015, $13,1-11$.

[78] Buerki-Thurnherr, T.; Xiao, L.; Diener, L.; Arslan, O.; Hirsch, C.; Maeder-Althaus, X.; Grieder, K.; Wampfler, B.; Mathur, S.; Wick, P. et al. In vitro mechanistic study towards a better understanding of zno nanoparticle toxicity. Nanotoxicology 2013, 7, 402-416.

[79] Multhoff, G.; Molls, M.; Radons, J. Chronic inflammation in cancer development. Frontiers in Immunology 2012, 2, 1-17.

[80] Poss, K. D.; Tonegawa, S. Reduced stress defense in heme oxygenase 1deficient cells. Proceedings of the National Academy of Sciences of the United States of America 1997, 94, 10925-10930. 
Tables and Figures
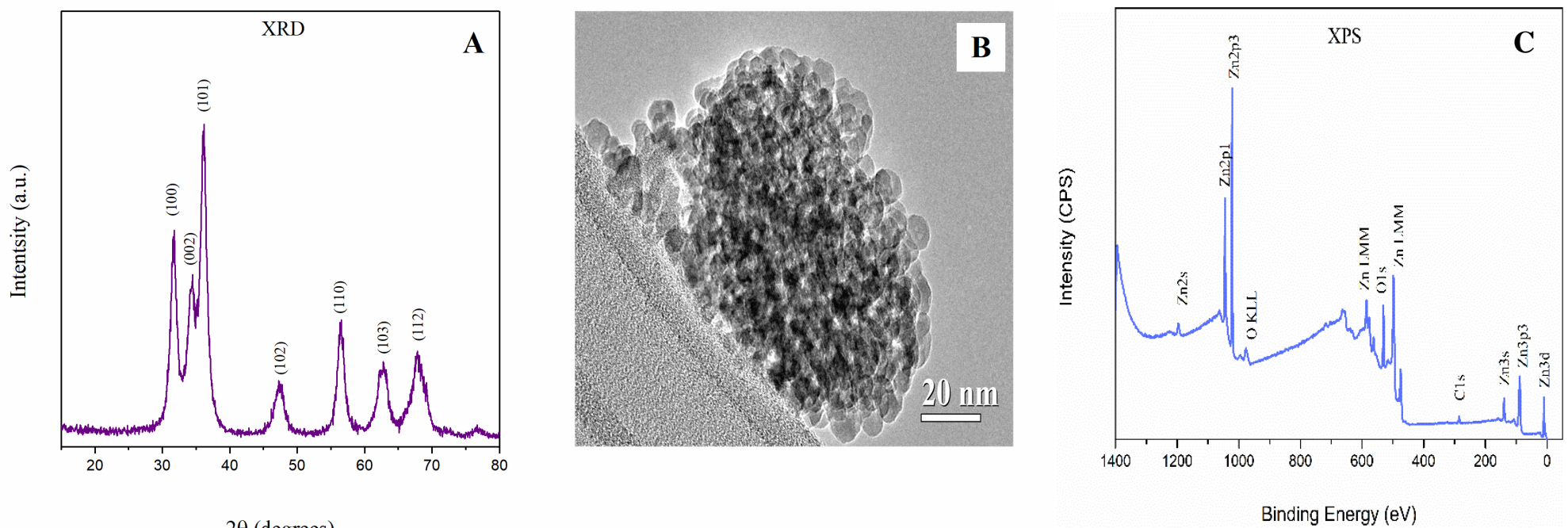

Figure 4.1 Size, morphology and composition analysis for the $\mathrm{nZnO}$. A) represents the XRD spectrum used to verify crystal structure and phase purity. TEM analysis (B) was employed to confirm NP morphology and average particle size. The atomic composition of the powered nanomaterial was determined using XPS (C). 

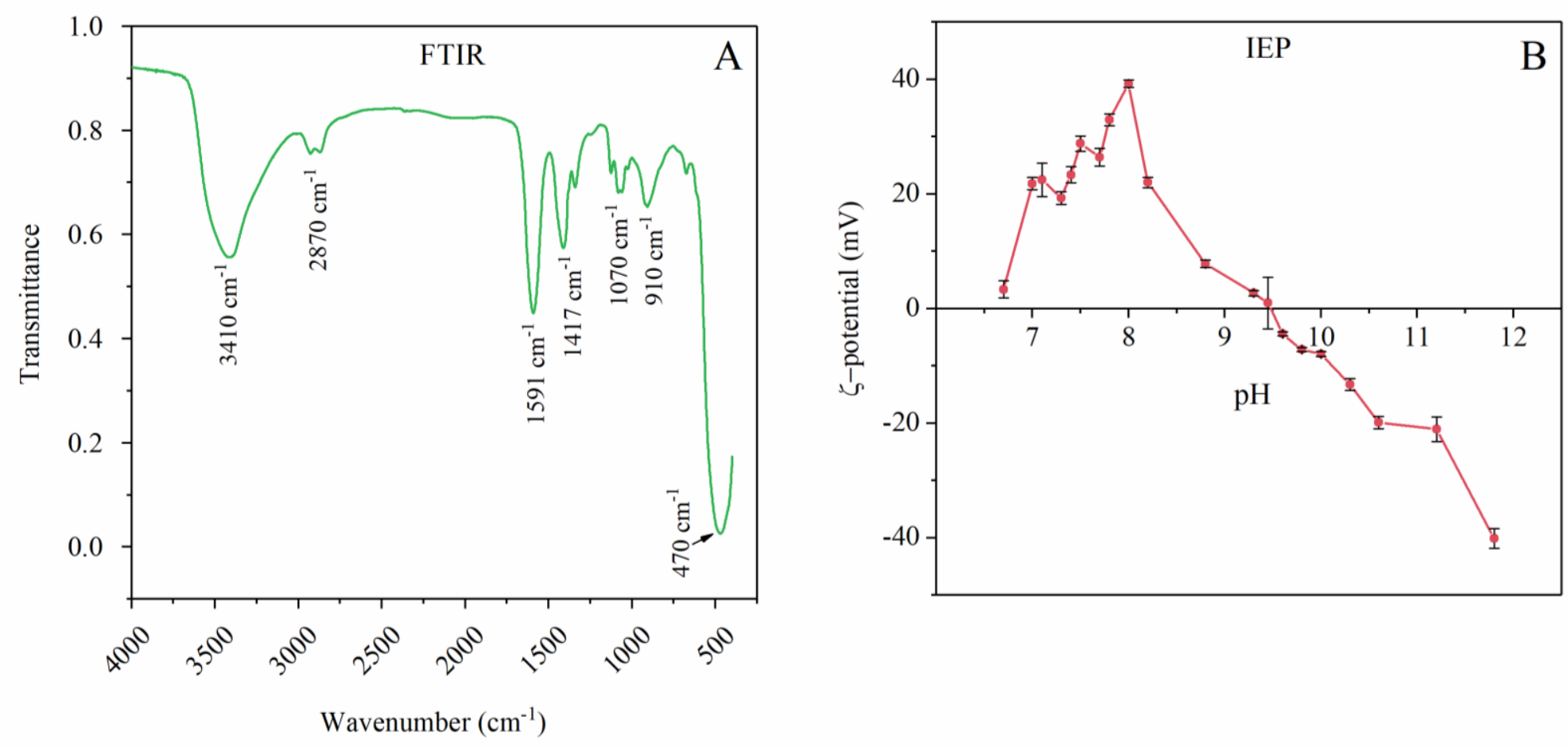

Figure 4.2 Surface structure analysis of the nZnO. A) Surface adsorbed groups were evaluated using FTIR and B) $\zeta$-potential and IEP were determined using DLS. 


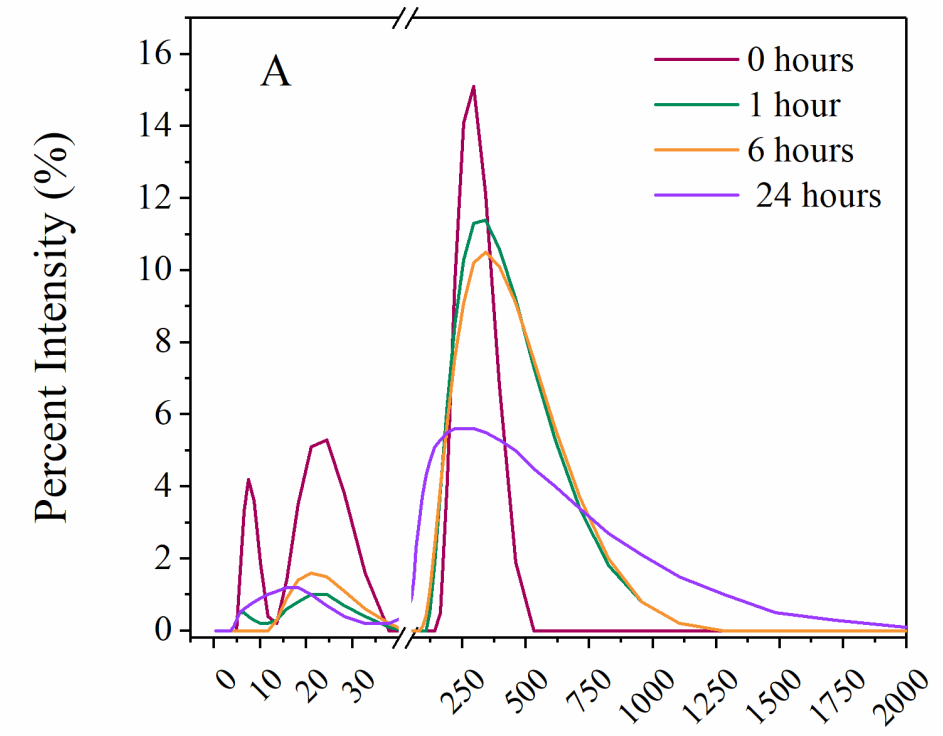

Hydrodynamic diameter (nm)

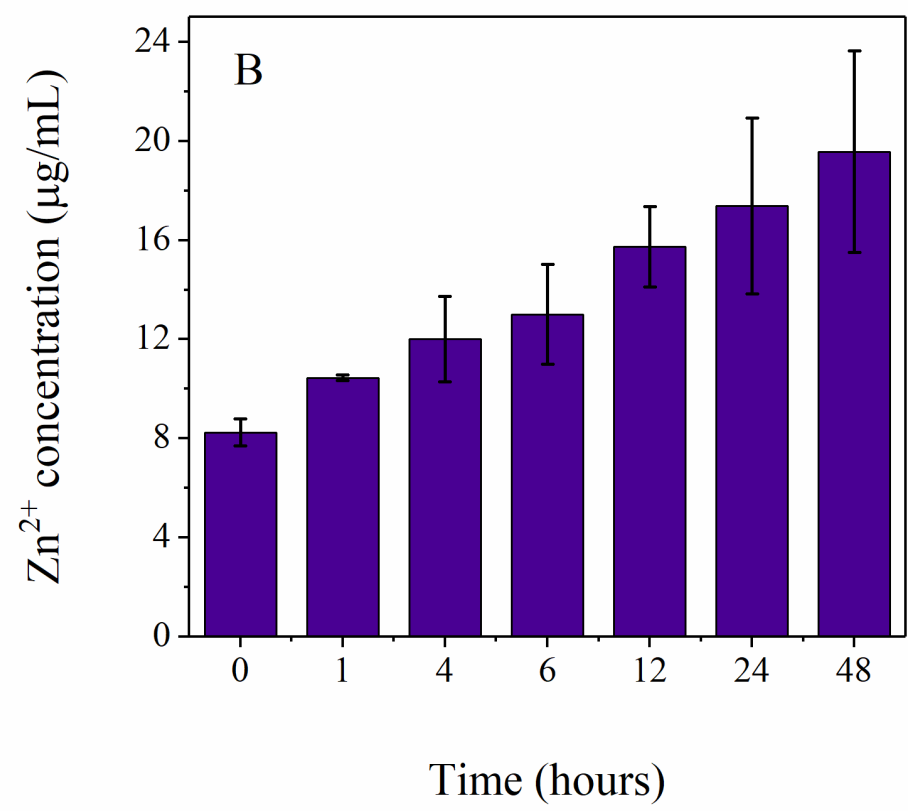

Time (hours)

Figure 4.3 The A) agglomeration tendencies and B) dissolution potential of $\mathrm{nZnO}$ dispersions were evaluated utilizing DLS measurements and ICP-MS, respectively. The $\mathrm{nZnO}$ dispersions utilized for both assays were created by introducing a $\mathrm{nZnO}$ FBS/PBS stock solution introduced to RPMI-based cellular media at a concentration of $486 \mu \mathrm{g} / \mathrm{mL}$. Error bars in (B) represent the standard deviation of $n=3$ replicates. 

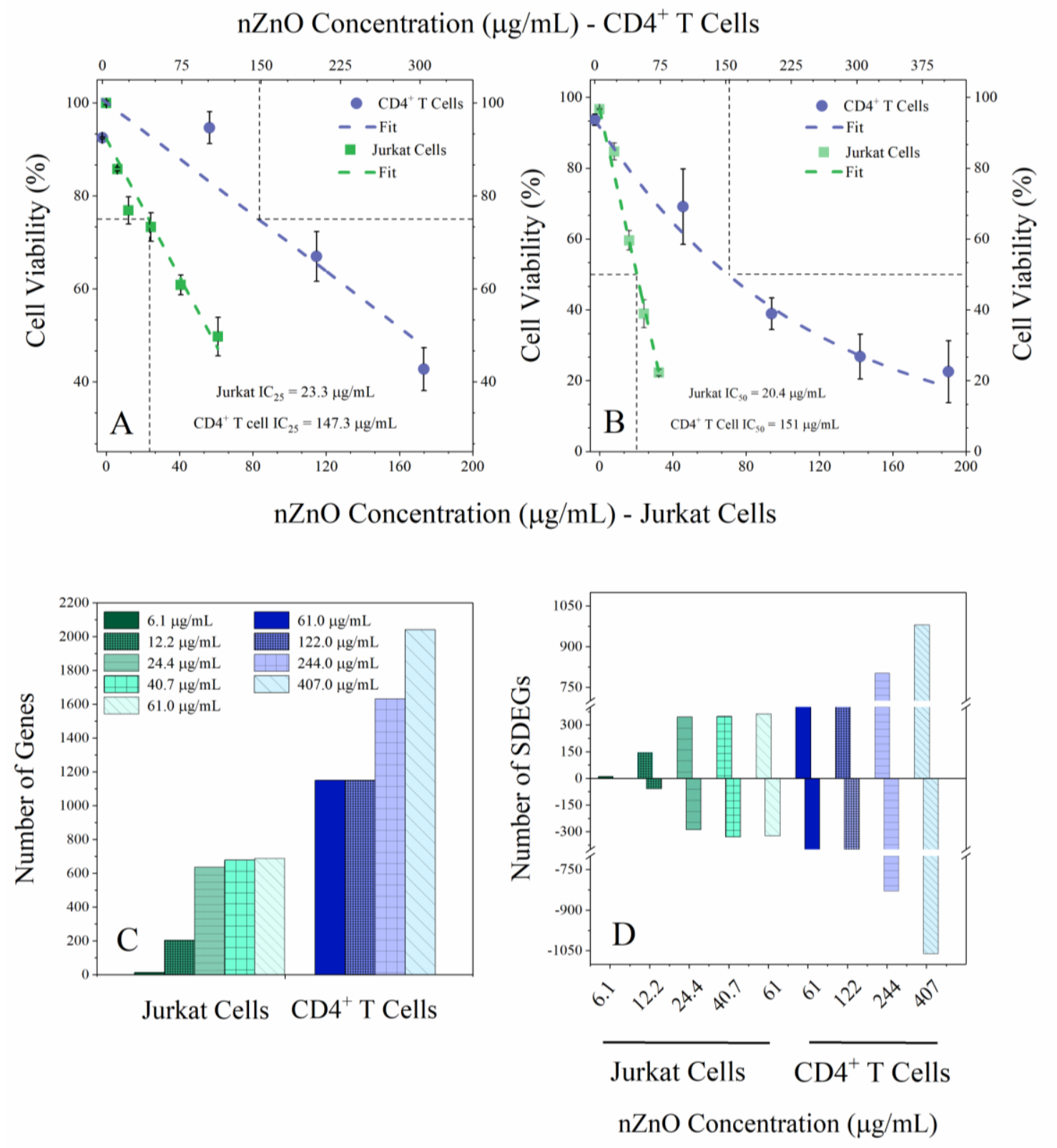

Figure 4.4 nZnO induced toxicity in Jurkat leukemic (green squares and line) and primary CD4 ${ }^{+} \mathrm{T}$ cells (blue circles and line) measured at A) 6 hours and B) 24 hours post treatment. The dashed lines in both plots represent the mathematical fit for the plotted data points. The bottom axis represents $\mathrm{nZnO}$ concentrations for Jurkat cells and the top axis depicts $\mathrm{nZnO}$ concentrations for the $\mathrm{CD4}^{+} \mathrm{T}$ cells. $\mathrm{C}$ ) The number of significantly differentially expressed genes for Jurkat leukemic (green patterned bars) and primary $\mathrm{CD4}^{+} \mathrm{T}$ cells (blue patterned bars) determined using cutoff values of (fold change) FC $>1.5$ and (false discovery rate) FDR $p<0.05$. D) The number of up- and down-regulated differentially expressed genes for Jurkat (green patterned bars) and primary $\mathrm{CD4}^{+} \mathrm{T}$ cells (blue patterned bars). Up-regulated genes are represented as positive values and down-regulated genes as negative values. 
Table 4.1 The number of SDEGs in each functional class. Functional classes were designated by Ingenuity Pathway Analysis (IPA) knowledge base. The yellow bars represented the $\mathrm{nZnO}$ treatment concentration closest to the $\mathrm{IC}_{50}$ for each cell type.

\begin{tabular}{|l|c|c|c|c|c|c|c|c|c|}
\hline Number of Genes & \multicolumn{4}{|c|}{ Jurkat } & \multicolumn{4}{c|}{ Primary } \\
\hline nZnO Concentration $(\mu \mathrm{g} / \mathrm{mL})$ & 6.1 & 12.2 & 24.4 & 40.7 & 61.0 & 61. & 122. & 244. & 407. \\
\hline Cytokine & & 1 & 1 & 2 & 3 & 16 & 14 & 15 & 16 \\
\hline Enzyme & 1 & 39 & 114 & 116 & 115 & 245 & 243 & 322 & 410 \\
\hline G-protein coupled receptor & & 2 & 6 & 6 & 6 & 11 & 14 & 19 & 21 \\
\hline Growth Factor & & 1 & 4 & 5 & 5 & 1 & 1 & 3 & 7 \\
\hline Ion channel & & 1 & 4 & 4 & 3 & 8 & 7 & 9 & 11 \\
\hline Kinase & & 3 & 18 & 19 & 20 & 59 & 56 & 186 & 101 \\
\hline Ligand-dependent nuclear receptor & & & & & & 1 & 1 & 1 & 1 \\
\hline Micro-RNA & & 1 & & & & & & 1 & 2 \\
\hline Other & 9 & 108 & 333 & 369 & 375 & 548 & 560 & 806 & 100 \\
\hline Peptidase & & 1 & 6 & 6 & 6 & 24 & 20 & 39 & 116 \\
\hline Phosphatase & & 7 & 11 & 14 & 12 & 18 & 17 & 26 & 30 \\
\hline Transcription Regulator & & 19 & 88 & 87 & 98 & 118 & 111 & 163 & 215 \\
\hline Translation regulator & & 1 & 4 & 4 & 5 & 9 & 8 & 16 & 17 \\
\hline Transmembrane Receptor & & 7 & 12 & 11 & 11 & 36 & 38 & 46 & 60 \\
\hline Transporter & 2 & 12 & 32 & 31 & 29 & 56 & 59 & 79 & 102 \\
\hline
\end{tabular}




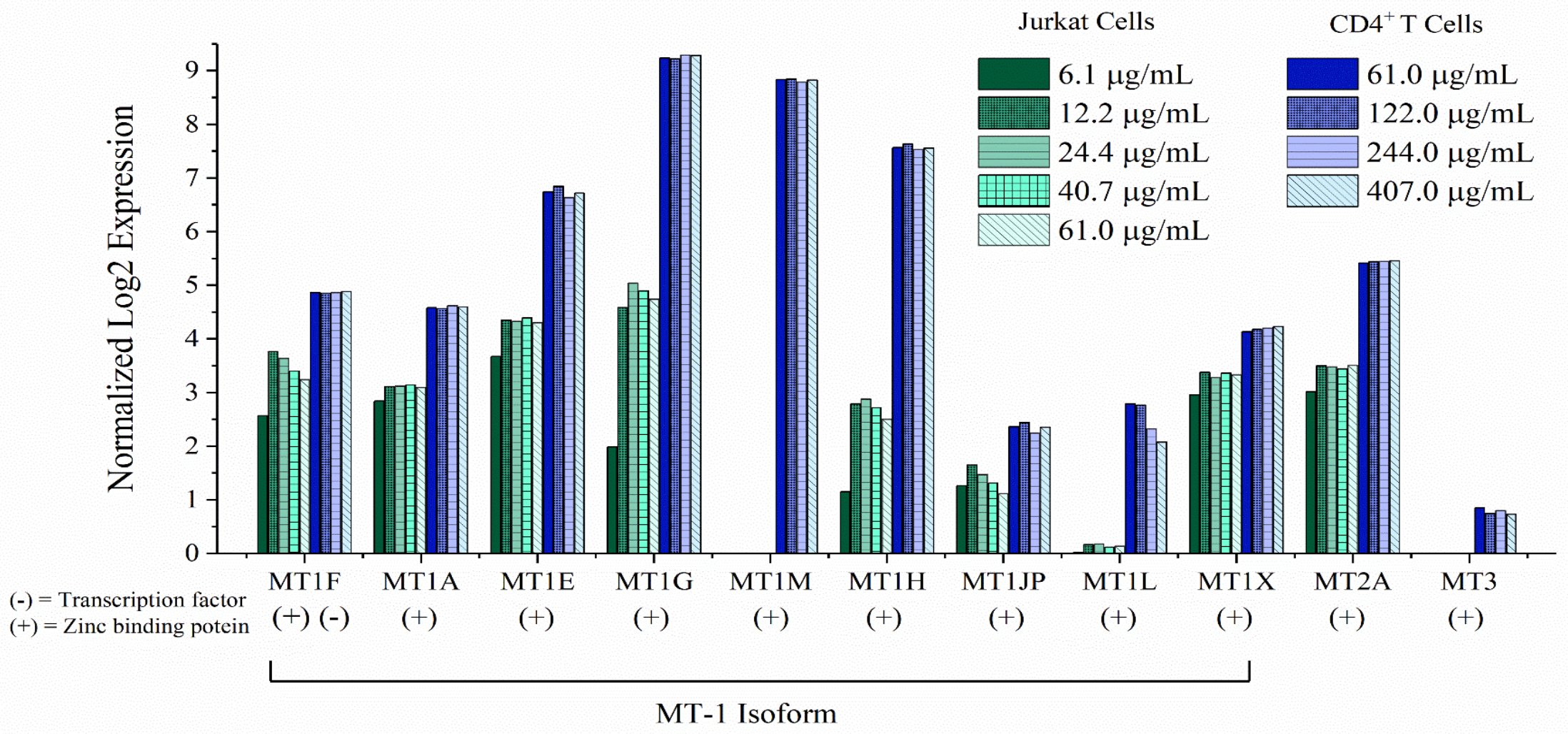

\section{Metallothionein Gene Family}

Figure 4.5 Differential expression of metallothionein genes in Jurkat and primary $\mathrm{CD4}^{+} \mathrm{T}$ cells treated with varying concentrations of $\mathrm{nZnO}$ for 6 hours. All displayed genes statistically significant $(\mathrm{FC}>1.5$ and FDR p $<0.05)$ and represent the average of four replicates. 


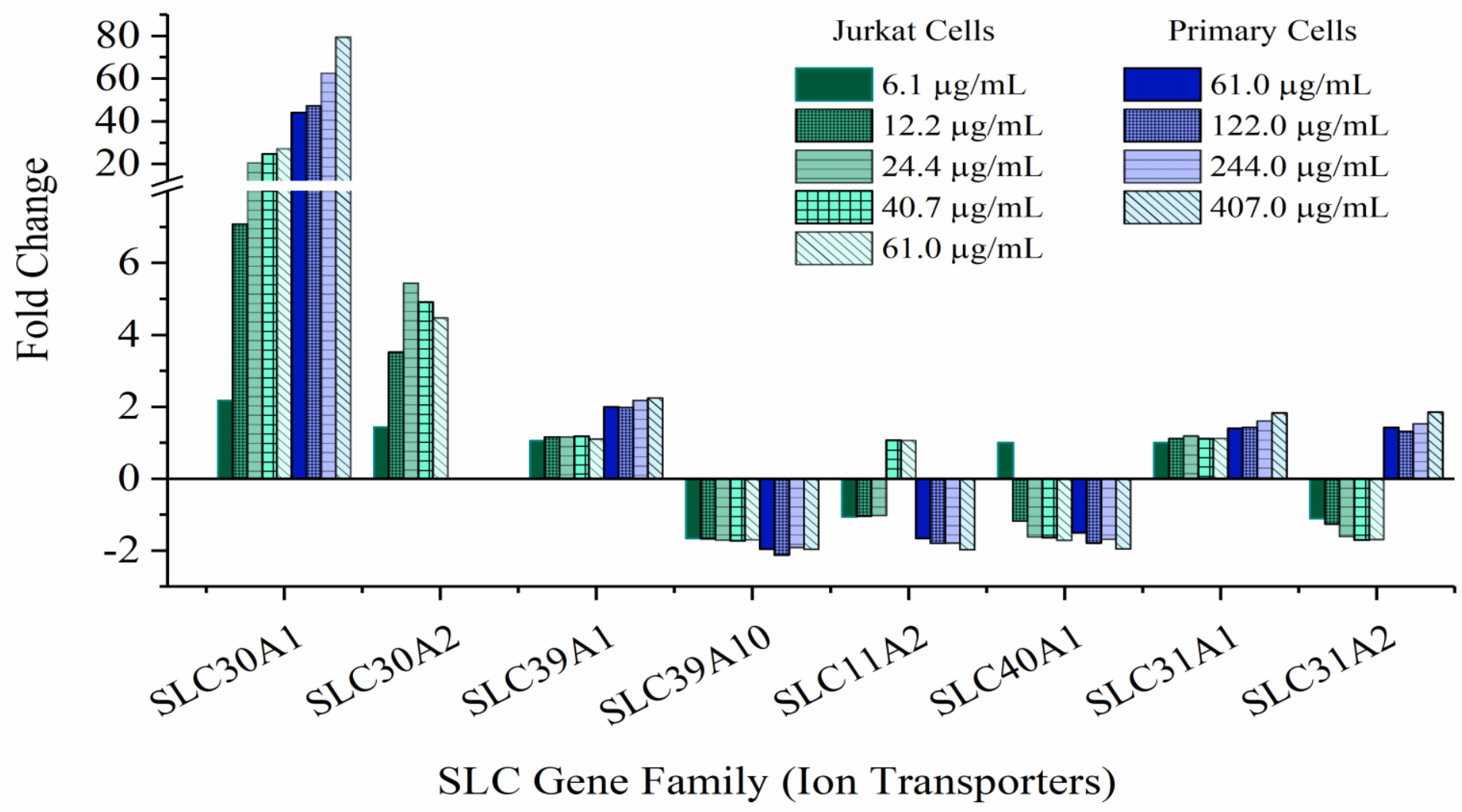

Figure 4.6 The expression of genes from the $S L C 30, S L C 39, S L C 31, S L C 11$ and $S L C 40$ transporter families in Jurkat and primary $\mathrm{CD4}^{+} \mathrm{T}$ cells treated with varying concentrations of $\mathrm{nZnO}$ for 6 hours. All displayed genes statistically significant $(\mathrm{FC}>1.5$ and FDR $\mathrm{p}<0.05)$ and represent the average of four replicates. 


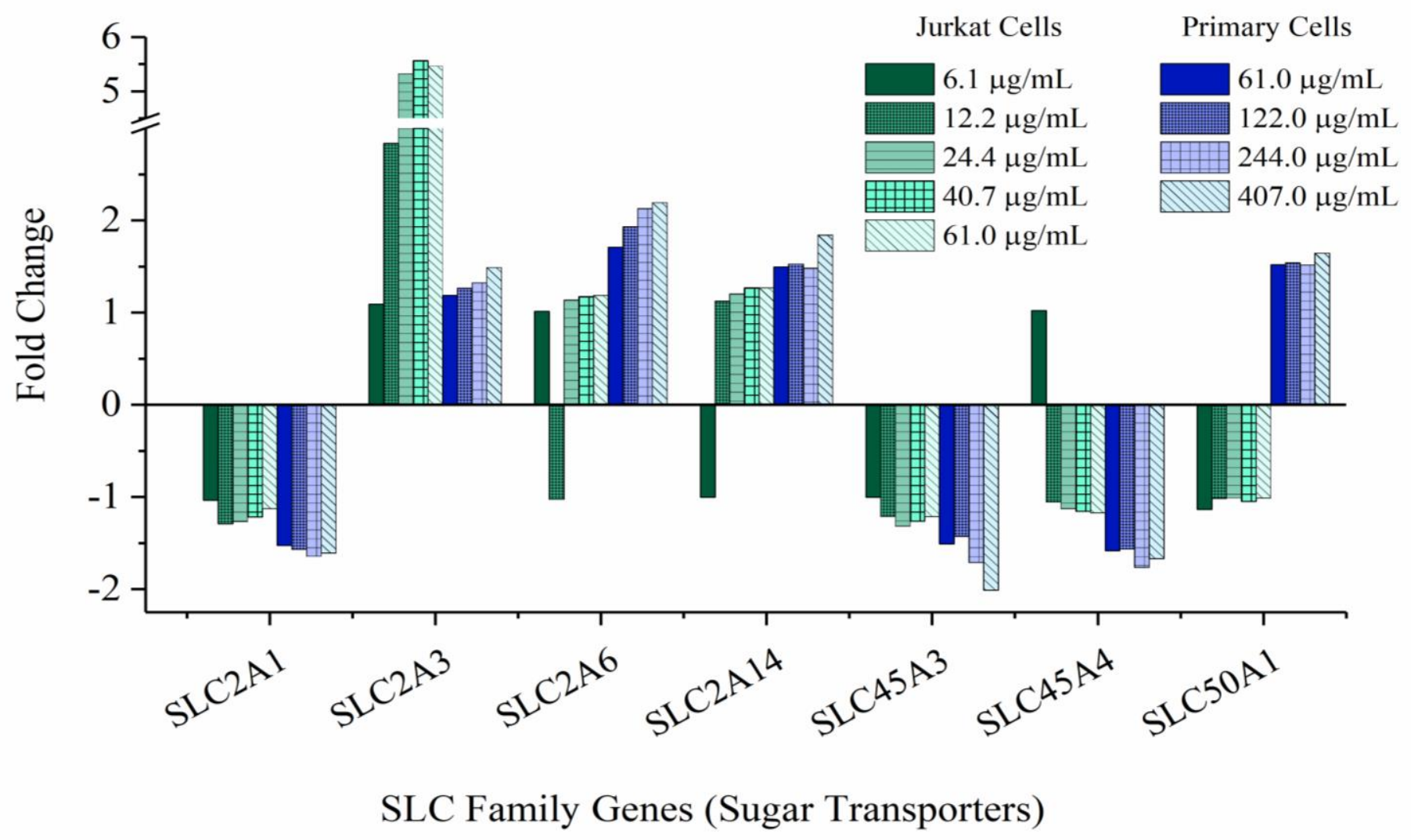

Figure 4.7 The expression of genes from the $S L C 2 A, S L C 45 A$, and $S L C 50 A$ transporter families in Jurkat and primary CD4 ${ }^{+}$ $\mathrm{T}$ cells treated with varying concentrations of $\mathrm{nZnO}$ for 6 hours. All displayed genes statistically significant (FC $>1.5$ and FDR $p<0.05)$ and represent the average of four replicates 


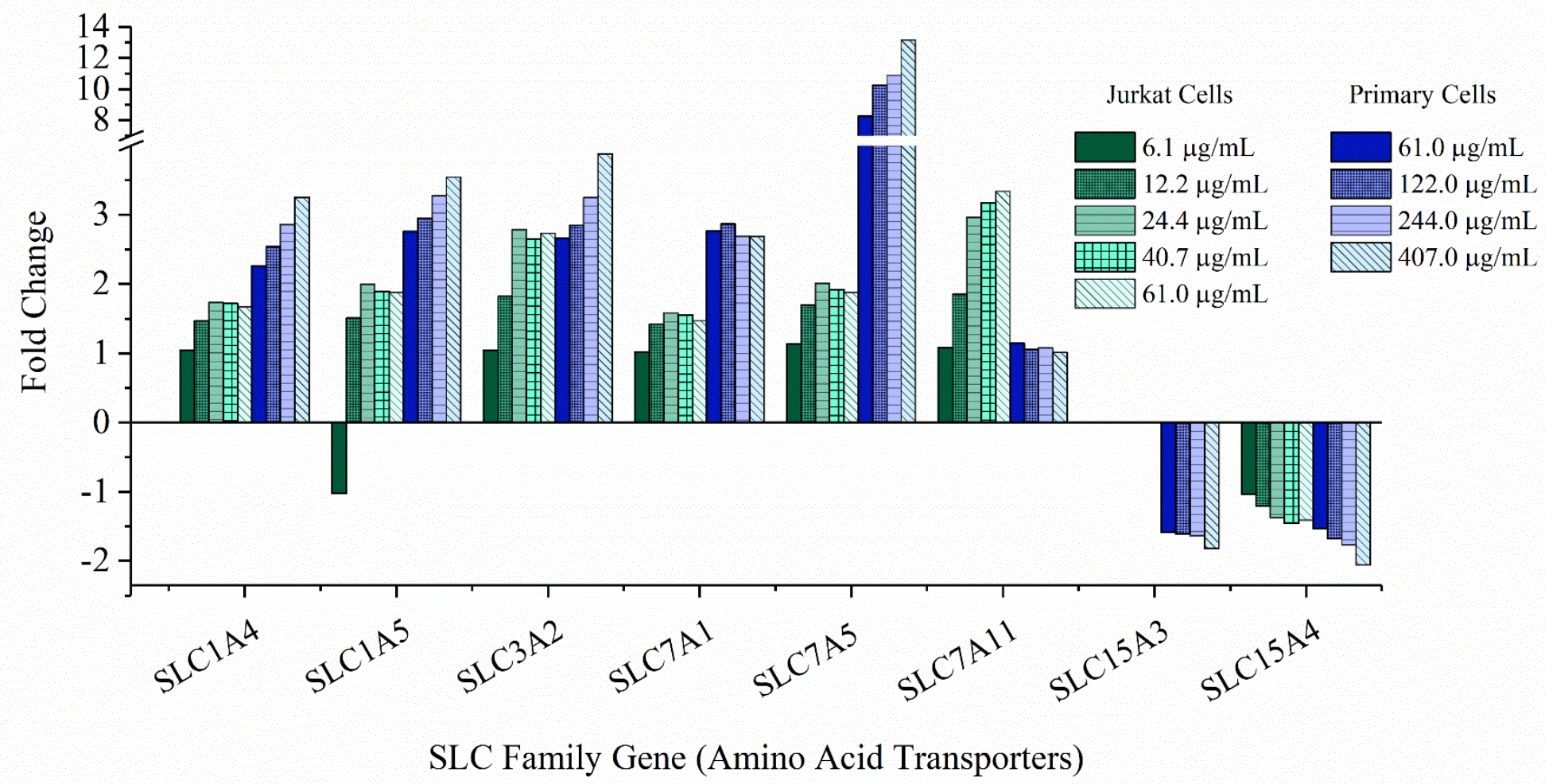

Figure 4.8 The expression of genes from the $S L C 1 A, S L C 3 A, S L C 7 A$, and $S L C 15 A$ transporter families in Jurkat and primary $\mathrm{CD4}^{+} \mathrm{T}$ cells treated with varying concentrations of $\mathrm{nZnO}$ for 6 hours. All displayed genes statistically significant (FC $>1.5$ and FDR $p<0.05$ ) and represent the average of four replicates 
Table 4.2 Fold change values for selected genes relating to inflammation, $T$ cell activation and cellular stress.

\begin{tabular}{|c|c|c|c|c|c|c|c|c|c|c|}
\hline \multirow{3}{*}{ Gene } & \multirow{3}{*}{ Description } & \multicolumn{9}{|c|}{ nZnO Treatment Concentration $(\mu \mathrm{g} / \mathrm{mL})$} \\
\hline & & \multicolumn{5}{|c|}{ Jurkat Cells } & \multicolumn{4}{|c|}{$\mathrm{CD4}^{+} \mathrm{T}$ cells } \\
\hline & & 6.1 & 12.2 & 24.4 & 40.7 & 61 & 61 & 122 & 244 & 407 \\
\hline \multicolumn{11}{|c|}{ Inflammatory Mediators } \\
\hline IL1A & Interleukin 1 alpha & & & & & & 1.684 & 1.434 & 1.55 & 1.516 \\
\hline IL1B & Interleukin 1 beta & & & & & & 3.687 & 2.623 & 2.602 & 2.49 \\
\hline TNF- $\alpha$ & Tumor necrosis factor & & & & & & 2.783 & 2.919 & 3.434 & 4.378 \\
\hline \multicolumn{11}{|c|}{ T Cell Activation } \\
\hline EGR1 & Early growth response & 1.034 & 1.285 & 2.826 & 3.809 & 4.601 & 1.766 & 1.885 & 2.315 & 2.835 \\
\hline CD69 & \multirow{3}{*}{ Activation genes } & -1.002 & 2.131 & 5.681 & 6.132 & 5.748 & 5.701 & 5.689 & 7.012 & 8.941 \\
\hline CTLA4 & & & & & & & -2.054 & -2.129 & -2.328 & -2.611 \\
\hline ICOS & & -1.02 & 1.551 & 1.509 & 1.413 & 1.363 & 1.111 & 1.081 & -1.001 & -1.041 \\
\hline
\end{tabular}

Table 4.2 Fold change values for selected genes relating to inflammation, $\mathbf{T}$ cell activation and cellular stress. 


\begin{tabular}{|c|c|c|c|c|c|c|c|c|c|c|}
\hline AARS & Alanyl-tRNA synthetase & 1.189 & 1.748 & 2.064 & 1.81 & 1.726 & 2.226 & 2.376 & 2.264 & 2.236 \\
\hline IARS & Isoleucyl-tRNA synthetase & 1.004 & 1.08 & 1.152 & 1.173 & 1.194 & 1.807 & 1.713 & 1.698 & 1.779 \\
\hline MARS & Methionyl-tRNA synthetase & -1.001 & 1.261 & 1.357 & 1.304 & 1.272 & 2.042 & 2.086 & 1.96 & 2.035 \\
\hline TARS & Threonyl-tRNA synthetase & 1.003 & 1.468 & 1.87 & 1.928 & 1.942 & 4.664 & 4.602 & 4.968 & 5.382 \\
\hline CARS & Cysteinyl-tRNA synthetase & -1.024 & 1.325 & 1.677 & 1.623 & 1.617 & 1.903 & 1.974 & 1.91 & 1.856 \\
\hline GARS & Glycyl-tRNA synthetase & 1.044 & 1.368 & 1.658 & 1.548 & 1.574 & 2.53 & 2.585 & 2.687 & 2.744 \\
\hline BIN1 & Bridging integrator 3 & & & & & & -2.029 & -1.841 & -2.112 & -1.903 \\
\hline TOB1 & $\begin{array}{l}\text { TNF superfamily member } \\
17\end{array}$ & -1.031 & 1.094 & 1.234 & 1.26 & 1.224 & -2.018 & -2.011 & -2.058 & -2.144 \\
\hline LTA & TNF- $\beta$ & 1.096 & 1.209 & 1.164 & 1.115 & 1.143 & 2.241 & 2.484 & 2.614 & 2.803 \\
\hline NFKB1A & $N F-\kappa \beta$ & -1.029 & 1.398 & 1.677 & 1.663 & 1.58 & 4.41 & 4.407 & 5.198 & 5.485 \\
\hline NFKBIB & \multirow{4}{*}{$N F-\kappa \beta$ inhibitors } & -1.022 & 1.102 & 1.188 & 1.175 & 1.146 & 1.881 & 2.045 & 2.422 & 2.842 \\
\hline NFKBID & & 1.007 & 1.025 & 1.138 & 1.193 & 1.172 & 1.66 & 1.8 & 2.22 & 2.748 \\
\hline NFKBIE & & -1.041 & -1.041 & 1.001 & 1.436 & 1.536 & 1.625 & 3.839 & 3.881 & 4.724 \\
\hline NFKBIZ & & -1.003 & 1.001 & 1.051 & 1.042 & 1.035 & 2.047 & 2.053 & 1.995 & 2.412 \\
\hline
\end{tabular}

Table 4.2 Fold change values for selected genes relating to inflammation, $\mathbf{T}$ cell activation and cellular stress. 


\begin{tabular}{|c|c|c|c|c|c|c|c|c|c|c|}
\hline NKIRAS1 & $\begin{array}{l}\text { NFKB inhibitor interacting } \\
\text { Ras }\end{array}$ & 1.012 & 1.234 & 1.774 & 1.824 & 1.93 & 1.709 & 1.742 & 2.109 & 2.418 \\
\hline REL & \multirow{2}{*}{$N F-\kappa \beta$ subunits } & 1.012 & 1.117 & 1.125 & 1.106 & 1.108 & 2.228 & 2.224 & 2.359 & 2.691 \\
\hline RELB & & 1.028 & 1.263 & 1.67 & 1.684 & 1.656 & 10.24 & 10.058 & 9.944 & 12.417 \\
\hline JUN & \multirow{5}{*}{$\begin{array}{l}\text { AP-1 transcription factor } \\
\text { subunits }\end{array}$} & 1.134 & 3.486 & 13.359 & 15.789 & 16.698 & 6.612 & 8.14 & 9.903 & 13.986 \\
\hline JUNB & & -1.03 & -1.125 & -1.035 & 1.057 & 1.098 & 1.112 & 1.073 & 1.337 & 1.818 \\
\hline JUND & & 1.226 & 1.972 & 3.405 & 3.54 & 3.629 & 1.301 & 1.441 & 1.663 & 1.712 \\
\hline FOS & & 1.01 & 1.776 & 8.55 & 14.441 & 20.317 & 14.999 & 18.153 & 28.127 & 48.1 \\
\hline FOSB & & 1.001 & 1.447 & 7.228 & 12.283 & 15.014 & 9.91 & 12.701 & 15.503 & 28.943 \\
\hline MYC & c-Myc transcription factor & 1.055 & -1.724 & -6.81 & -9.666 & -10.705 & -1.679 & -1.839 & -2.291 & -2.99 \\
\hline LCK & LCK proto-oncogene & -1.062 & -1.093 & -1.027 & 1.003 & 1.062 & -1.203 & -1.232 & -1.269 & -1.194 \\
\hline LAG3 & Lymphocyte activating 3 & & & & & & -1.857 & -1.665 & -1.796 & -1.779 \\
\hline RAC1 & Ras-related gene & -1.007 & -1.014 & -1.041 & -1.092 & -1.106 & -1.234 & -1.226 & -1.253 & -1.363 \\
\hline RAC2 & Ras-related gene & 1.039 & -1.138 & -1.287 & -1.317 & -1.227 & -1.393 & -1.366 & -1.344 & -1.401 \\
\hline RASA3 & Ras-related gene & -1.012 & -1.105 & -1.099 & -1.063 & -1.039 & -1.543 & -1.515 & -1.727 & -1.686 \\
\hline
\end{tabular}

Table 4.2 Fold change values for selected genes relating to inflammation, $T$ cell activation and cellular stress. 


\begin{tabular}{|c|c|c|c|c|c|c|c|c|c|c|}
\hline RASD1 & Ras-related gene & 1.066 & 1.115 & 1.379 & 1.668 & 1.925 & 1.844 & 2.103 & 2.778 & 3.414 \\
\hline SLAMF1 & $\begin{array}{l}\text { Signaling lymphocytic } \\
\text { activation molecule }\end{array}$ & & & & & & -1.303 & -1.386 & -1.456 & -1.747 \\
\hline CABIN1 & $\begin{array}{l}\text { Calcineurin binding protein } \\
1\end{array}$ & 1.06 & -1.027 & 1.011 & -1.001 & 1.035 & -2.318 & -2.128 & -2.377 & -2.448 \\
\hline CALR & calreticulin & -1.048 & -1.203 & -1.144 & -1.06 & -1.087 & 1.421 & 1.36 & 1.493 & 1.505 \\
\hline CAMK2G & \multirow{4}{*}{$\begin{array}{l}\text { Calcium/calmodulin protein } \\
\text { related genes }\end{array}$} & -1 & -1.276 & -1.236 & -1.138 & -1.076 & -2.033 & -2.263 & -2.594 & -2.976 \\
\hline CAMK2N1 & & 1.044 & 1.113 & 1.123 & 1.09 & 1.118 & 1.16 & 1.244 & 1.338 & 1.344 \\
\hline CAMK4 & & & & & & & 1.12 & 1.067 & 1.161 & 1.168 \\
\hline CAMSAP1 & & 1.059 & -1.009 & -1.012 & -1.02 & 1.028 & -1.551 & -1.598 & -1.643 & -1.833 \\
\hline CALHM2 & $\begin{array}{l}\text { Calcium homeostasis } \\
\text { modulator }\end{array}$ & -1.08 & -1.048 & -1.058 & -1.069 & -1.014 & -1.767 & -1.778 & -1.818 & -1.758 \\
\hline PMAIP1 & $\begin{array}{l}\text { Pro-apoptotic member of } \\
\text { the Bcl-2 protein family }\end{array}$ & 1.06 & 2.238 & 3.47 & 3.562 & 3.444 & 10.743 & 10.827 & 12.349 & 16.39 \\
\hline PIM2 & \multirow{2}{*}{$\begin{array}{l}\text { Pim-2 proto-oncogenes, } \\
\text { serine/threonine kinase }\end{array}$} & -1.093 & -1.255 & -1.098 & 1.047 & 1.053 & 1.685 & 1.64 & 1.822 & 1.955 \\
\hline PIM3 & & -1.033 & -1.199 & -1.173 & -1.173 & -1.101 & 2.168 & 2.334 & 2.881 & 3.866 \\
\hline
\end{tabular}

Table 4.2 Fold change values for selected genes relating to inflammation, $T$ cell activation and cellular stress. 


\begin{tabular}{|c|c|c|c|c|c|c|c|c|c|c|}
\hline MAP3K4 & MEKK4 & 1.023 & -1.188 & -1.172 & -1.093 & -1.077 & -1.471 & -1.485 & -1.562 & -1.598 \\
\hline MAP3K5 & ASK1 & 1.026 & -1.047 & -1.086 & -1.077 & -1.041 & -1.61 & -1.622 & -1.73 & -1.897 \\
\hline MAP3K6 & ASK2 & -1.015 & 1.026 & 1.047 & 1.093 & 1.116 & -1.522 & -1.539 & -1.588 & -1.682 \\
\hline MAP3K8 & tpl-2, TPL2, COT, AURA2 & 1.056 & 1.428 & 1.19 & 1.046 & 1.023 & 2.402 & 2.118 & 2.104 & 1.979 \\
\hline MAP4K1 & HPK1 & 1.004 & 1.006 & 1.128 & 1.124 & 1.102 & -1.964 & -1.857 & -2.033 & -2.016 \\
\hline MAPKAPK3 & & 1.003 & -1.215 & -1.195 & -1.199 & -1.194 & -1.412 & -1.383 & -1.592 & -1.629 \\
\hline \multicolumn{11}{|c|}{ Stress Induced Protein } \\
\hline HSPA6 & \multirow{3}{*}{$\begin{array}{l}\text { Heat shock protein family } \\
\text { (Hsp70) } \\
\text { Heat shock protein family } \\
\text { (Hsp70) }\end{array}$} & 1.055 & 8.429 & 38.521 & 43.314 & 49.866 & 34.024 & 41.029 & 57.326 & 96.021 \\
\hline $\begin{array}{l}\text { HSPA1A/ } \\
\text { HSPA1B }\end{array}$ & & 1.38 & 11.343 & 18.903 & 17.874 & 19.273 & 24.557 & 28.633 & 34.486 & 38.712 \\
\hline HSPA7 & & 1.022 & 3.033 & 11.044 & 14.15 & 14.957 & 7.322 & 8.909 & 10.497 & 19.054 \\
\hline HSPH1 & $\begin{array}{l}\text { Heat shock protein family } \\
\text { (Hsp110) }\end{array}$ & 1.51 & 3.729 & 4.856 & 4.852 & 4.717 & 13.155 & 14.108 & 17.1 & 19.917 \\
\hline HSPE1 & $\begin{array}{l}\text { Heat shock protein family } \\
\text { (Hsp10) }\end{array}$ & 1.042 & 1.395 & 1.538 & 1.528 & 1.543 & 5.525 & 5.823 & 6.172 & 7.821 \\
\hline
\end{tabular}


Table 4.2 Fold change values for selected genes relating to inflammation, $\mathbf{T}$ cell activation and cellular stress.

\begin{tabular}{|l|l|l|l|l|l|l|l|l|l|l|}
\hline \multirow{2}{*}{ DNAJB1 } & & -1.005 & 5.927 & 13.259 & 13.742 & 13.743 & 4.372 & 4.827 & 6.73 & 8.526 \\
\hline DNAJA1 & $\begin{array}{l}\text { DnaJ heat shock protein } \\
\text { family (Hsp40) }\end{array}$ & 1.172 & 1.615 & 1.878 & 1.818 & 1.859 & 5.663 & 5.859 & 6.606 & 7.612 \\
\cline { 3 - 11 } DNAJB4 & & 1.028 & 1.48 & 1.967 & 2.02 & 1.792 & 4.253 & 4.029 & 5.396 & 6.926 \\
\hline HSPB1 & Heat shock protein family B & 1.035 & 1.343 & 1.543 & 1.527 & 1.462 & 4.943 & 5.819 & 6.077 & 6.857 \\
\hline STIP1 & $\begin{array}{l}\text { Stress induced } \\
\text { phosphoprotein 1 }\end{array}$ & 1.153 & 1.781 & 2.258 & 2.03 & 2.076 & 4.287 & 4.974 & 5.322 & 6.758 \\
\hline HMOX1 & Heme oxygenase 1 & 1.03 & 1.262 & 2.073 & 2.205 & 2.368 & 54.142 & 58.963 & 76.719 & 84.388 \\
\hline HIF1A & Hypoxia inducible factor & 1.031 & -1.006 & -1.086 & -1.066 & -1.061 & 1.965 & 1.804 & 2.043 & 2.138 \\
\hline DRAM1 & $\begin{array}{l}\text { DNA damage regulated } \\
\text { autophagy modulator 1 }\end{array}$ & & & & & & 1.625 & 1.615 & 1.63 & 1.844 \\
\hline SQSTM1 & Sequestosome 1 & -1.023 & 1.125 & 1.281 & 1.323 & 1.297 & 3.236 & 3.408 & 3.784 & 4.046 \\
\hline
\end{tabular}




\section{CHAPTER 5 CONCLUSIONS}

$\mathrm{nZnO}$ has a promising future in biomedical applications if clear mechanisms of toxicity can be identified. This study sought to explore these potential mechanisms from two distinct perspectives. First, we explored how the physicochemical properties of $\mathrm{nZnO}$ influence NP-induced cytotoxicity and cellular oxidative stress. Secondly, we analyzed the cellular transcriptome changes in response to $\mathrm{nZnO}$ treatment to potentially elucidate cellular processes that contribute to $\mathrm{nZnO}$ selective toxicity towards cancer cells.

Several important findings reveal that the physicochemical properties of $\mathrm{nZnO}$ influence NP-induced toxicity. The agglomeration and sedimentation behavior of unstable NP dispersions adversely alters the results of downstream biological assays. Obtaining stable $\mathrm{nZnO}$ dispersions with the use of FBS proteins significantly changes the assay results in both suspension and adherent cell models compared to findings with unstable dispersions. These results confirm our hypothesis that achieving stable NP dispersion stability is a critical factor in obtaining reliable and reproducible findings.

When the material properties of a panel of differently synthesized $\mathrm{nZnO}$ were analyzed, the synthesis method was identified as a contributing factor to cytotoxicity. Indeed, $\mathrm{nZnO}$ synthesized using wet chemical methods demonstrates greater surface reactivity, extracellular dissolution potential and significantly greater cytotoxicity than nZnO synthesized through high temperature methods. Additionally, our results underscore the importance of extensive material characterization as no one specific physicochemical 
property was found to contribute solely to the observed cytotoxicity. Rather a combination of material properties appears to influence the toxic potential of $\mathrm{nZnO}$.

Finally, preliminary analysis of the transcriptome changes in cancerous and primary $\mathrm{T}$ cells post-treatment with $\mathrm{nZnO}$ reveal several potential gene targets that may regulate the selective toxicity of $\mathrm{nZnO}$ against cancer cells. Significant differences between the cancer and primary $\mathrm{T}$ cells are present in the expression of genes involved in the regulation of zinc homeostasis, ion transport, cellular metabolism, inflammation, $\mathrm{T}$ cell activation, and cellular stress responses. Additional experiments are needed to verify these initial findings and confirm the importance of these transcriptome changes in the preferentially selectively of $\mathrm{nZnO}$ towards cancer cells.

Together, the results of this dissertation confirm that $\mathrm{nZnO}$ toxicity is strongly influenced by the NP fabrication methods, material properties and differential cellular responses. Further studies are needed to connect specific material properties to unique cellular responses and mechanisms of cell death. This knowledge would potentially enable the development of a "design rule" for the fabrication of nZnO to achieve environmentally safe NPs for consumer products and NPs with maximal selective toxicity for use in alternative cancer therapeutics. 


\section{APPENDIX}




\section{Copyright Permissions}

\section{Nature Publishing Group License Terms and Conditions}

This Agreement between Catherine Anders ("You") and Nature Publishing Group ("Nature Publishing Group") consists of your license details and the terms and conditions provided by Nature Publishing Group and Copyright Clearance Center.

License Number

License date

Licensed Content Publisher

Licensed Content Publication

Licensed Content Title

Licensed Content Author

Licensed Content Date

Licensed Content Volume

Licensed Content Issue

Type of Use

Requestor type

Format

Portion

Figures

Article: Role of Zinc Signaling In The Immune System
4180590061547

Sep 02, 2017

Nature Publishing Group

Nature Nanotechnology

The effect of sedimentation and diffusion on

cellular uptake of gold nanoparticles

Eun Chul Cho, Qiang Zhang, Younan Xia

Apr 24, 2011

6

6

reuse in a dissertation / thesis

academic/educational

print and electronic

Figures/tables/illustrations

Figure 4 | Different zones involved in cellular uptake of gold 


\author{
Author: $\quad$ Shintaro Hojyo and Toshiyuki Fukada2 \\ Publication: Journal of Immunology Research \\ Publisher: Hindawi Publishing Corporation \\ Date: $\quad 2016$ \\ Copyright $\odot$ 2016, Hojyo and Fukada \\ Use: Figure 2 (Figure 1.13 in document)
}

\title{
Open Access
}

This article is distributed under the terms of the Creative Commons Attribution 4.0 International License (http://creativecommons.org/licenses/by/4.0/), which permits unrestricted use, distribution, and reproduction in any medium, provided you give appropriate credit to the original author(s) and the source, provide a link to the Creative Commons license, and indicate if changes were made.

Article: Serum Proteins Enhance Dispersion Stability and Influence the Cytotoxicity and Dosimetry of ZnO Nanoparticles in Suspension and Adherent Cancer Cell Models

\section{Copyright Permission}


Author: $\quad$ Catherine B. Anders

Publication: Nanoscale Research Letters

Publisher: $\quad$ Springer

Date: $\quad$ Jan 1,2015

Copyright (C) 2015, Anders et al.

\section{Open Access}

This article is distributed under the terms of the Creative Commons Attribution 4.0 International License (http://creativecommons.org/licenses/by/4.0/), which permits unrestricted use, distribution, and reproduction in any medium, provided you give appropriate credit to the original author(s) and the source, provide a link to the Creative Commons license, and indicate if changes were made. 


\section{Coauthor Permission to Publish in Dissertation}

Catherine Binns Anders

catherineanders@boisestate.edu

$11 / 15 / 2017$

Dear Jordan:

I am writing to request permission to use the article "Serum Proteins Enhance Dispersion Stability and Influence the Cytotoxicity and Dosimetry of $\mathrm{ZnO}$ Nanoparticles in Suspension and Adherent Cancer Cell Models," which was published in Nanoscale Research Letters 10(448), on November 17, 2015 for which you were a contributing author, in my dissertation.

I will include acknowledgements and/or appropriate citations to the work and copyright and reprint rights in the appendices. The bibliographic citation will appear in the References list at the end of the manuscript as typed below. Please advise me of any changes you require.

Please indicate your approval of this request by signing in the space provided, attaching any forms or instructions necessary to confirm permission. If you charge a reprint fee for use of your material, please indicate that as well. If you have questions, please email me.

I hope you will be able to reply immediately. If you are not the copyright holder, please forward my request to the appropriate person or institution.

Thank you for your cooperation,

Catherine Binns Anders

I hereby give permission to Catherine Binns Anders to reprint the following material in her dissertation.

Anders, C. B.; Chess, J. J.; Wingett, D. G.; Punnoose, A. Serum proteins enhance dispersion stability and influence the cytotoxicity and dosimetry of zno nanoparticles in suspension and adherent cancer cell models. Nanoscale Research Letters 2015, 10.

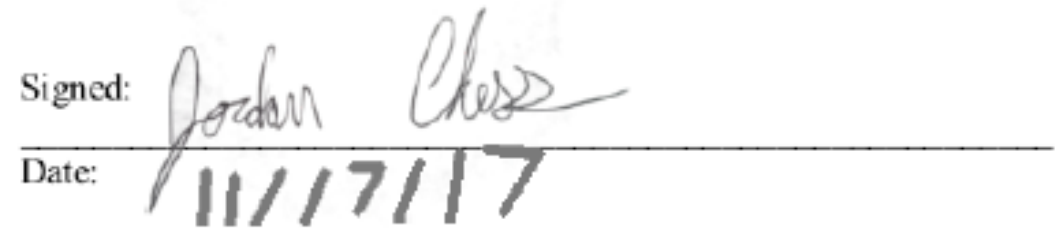


Article: ZnO Nanoparticle Preparation Route Influences Surface Reactivity, Dissolution and Cytotoxicity

\section{Copyright Permission}

Author: $\quad$ Catherine B. Anders

Publication: Environmental Science: Nano

Publisher: Royal Society of Chemistry

Date: $\quad$ January 5,2018

Copyright $\odot$ 2018, Anders et al.

\section{Coauthor Permission to Publish in Dissertation}

Catherine Binns Anders

catherineanders@boisestate.edu

$1 / 14 / 2018$

Dear Coauthor:

I am writing to request permission to use the article " $\mathrm{ZnO}$ Nanoparticle Preparation Route Influences Surface Reactivity, Dissolution and Cytotoxicity," which was published in Environmental Science: Nano, DOI: 10.1039/C7EN00888K, on January 5, 2018 for which you were a contributing author, in my dissertation.

I will include acknowledgements and/or appropriate citations to the work and copyright and reprint rights in the appendices. The bibliographic citation will appear in the References list at the end of the manuscript as typed below. Please advise me of any changes you require. 
Please indicate your approval of this request by signing in the space provided, attaching any forms or instructions necessary to confirm permission. If you charge a reprint fee for use of your material, please indicate that as well. If you have questions, please email me.

I hope you will be able to reply immediately. If you are not the copyright holder, please forward my request to the appropriate person or institution.

Thank you for your cooperation, Catherine Binns Anders

I hereby give permission to Catherine Binns Anders to reprint the following material in her dissertation.

Anders, C.B., Eixenberger, J.E., Franco, N.A., Hermann, R.J., Rainey, K.D., Chess, J.J., Punnoose, A., and Wingett, D.G. ZnO Nanoparticle Preparation Route Influences Surface Reactivity, Dissolution and Cytotoxicity. Environmental Science: Nano 2018, DOI: 10.1039/C7EN00888K.

Signed:

Printed:

Date: 
I hereby give permission to Catherine Binns Anders to reprint the following material in her dissertation.

Anders, C.B., Eixenberger, J.E., Franco, N.A., Hermann, R.J., Rainey, K.D., Chess, J.J., Punnoose, A., and Wingett, D.G. ZnO Nanoparticle Preparation Route Influences Surface Reactivity, Dissolution and Cytotoxicity. Environmental Science: Nano 2018, DOI: 10.1039/C7EN00888K.

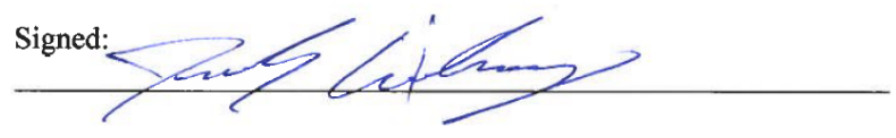

Printed: JoSH EIXENBERGER

Date: $\quad 1-22-18$

I hereby give permission to Catherine Binns Anders to reprint the following material in her dissertation.

Anders, C.B., Eixenberger, J.E., Franco, N.A., Hermann, R.J., Rainey, K.D., Chess, J.J., Punnoose, A., and Wingett, D.G. ZnO Nanoparticle Preparation Route Influences Surface Reactivity, Dissolution and Cytotoxicity. Environmental Science: Nano 2018, DOI: 10.1039/C7EN00888K.

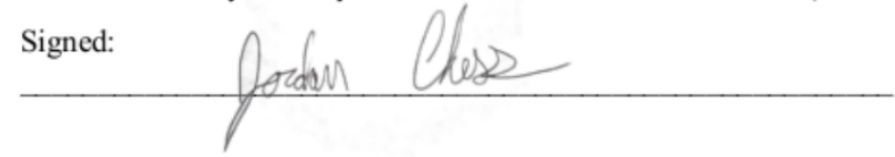

Printed: JordanChess

Date: $1 / 14 / 2018$

I hereby give permission to Catherine Binns Anders to reprint the following material in her dissertation.

Anders, C.B., Eixenberger, J.E., Franco, N.A., Hermann, R.J., Rainey, K.D., Chess, J.J., Punnoose, A., and Wingett, D.G. ZnO Nanoparticle Preparation Route Influences Surface Reactivity, Dissolution and Cytotoxicity. Environmental Science: Nano 2018, DOI: 10.1039/C7ENO0888K.

Signed: RHermann

Printed:
Rebecea Hermann

Date: 01.16 .18 
I hereby give permission to Catherine Binns Anders to reprint the following material in her dissertation.

Anders, C.B., Eixenberger, J.E., Franco, N.A., Hermann, R.J., Rainey, K.D., Chess, J.J., Punnoose, A., and Wingett, D.G. ZnO Nanoparticle Preparation Route Influences Surface Reactivity, Dissolution and Cytotoxicity. Environmental Science: Nano 2018, DOI: 10.1039/C7EN00888K.

Signed:

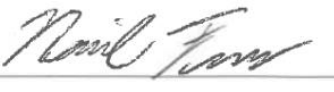

Printed:

Date: $1 \cdot 18 \cdot 2018$

I hereby give permission to Catherine Binns Anders to reprint the following material in her dissertation.

Anders, C.B., Eixenberger, J.E., Franco, N.A., Hermann, R.J., Rainey, K.D., Chess, J.J., Punnoose, A., and Wingett, D.G. ZnO Nanoparticle Preparation Route Influences Surface Reactivity, Dissolution and Cytotoxicity. Environmental Science: Nano 2018. DOI: 10.1039/C7EN00888:

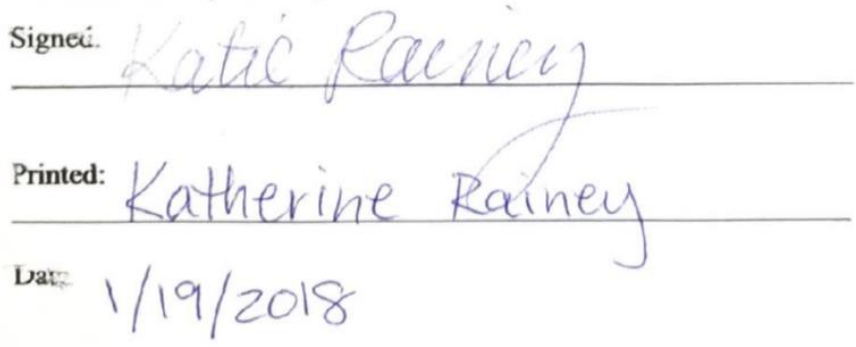

Note:

The coauthor Alex Punnoose passed away during the preparation of this manuscript. 